\title{
THE MANUFACTURE OF DISCONNECTION
}

by

Alex Beattie

A thesis

submitted to Te Herenga Waka-Victoria of University of Wellington in fulfilment of the requirements for the degree of Doctor of Philosophy

School of English, Film, Theatre and Media Studies, and Art History Faculty of Humanities and Social Sciences

Te Herenga Waka-Victoria of University of Wellington 

In loving memory of my father and grandfather

Matthew Beattie, 1954-2019

Mike Goddard, 1927-2019 


\section{Abstract}

In Silicon Valley, the world's most famous site of technological innovation, technology professionals are rejecting their own inventions and disconnecting from the internet. According to reports, technologists believe their designs and algorithms "hijack" user's brains (Lewis 2017) and executives are sending their children to technology-free schools (Jenkin 2015). These technologists are not disillusioned by digital technology per se, but rather by the ideological and socio-economic system underpinning digital technology. This system is the 'attention economy' where media companies, advertisers and technology platforms compete for end user attention (Crogan and Kinsley 2012), which in turn incentivises technologists to create compulsive experiences for users to maximise time spent on device (Lanier 2018). In response to concerns about the attention economy, some technologists have become 'disconnectionists'-opponents to the culture of constant connection they helped create (Jurgenson 2013). Not only are they disconnecting from their own inventions but, in true Silicon Valley style, are also inventing new technology-based ways to disconnect from the internet ("technologies of disconnection"). In other words, these disconnectionists are manufacturing disconnection.

This research investigates the manufacture of disconnection as a mode of resistance to the attention economy. I contend that the manufacture of disconnection does not separate the user from the internet, but rather deploys technical and social practices to reorganise user relations to themselves and the internet in order to resist the attention economy. I critically assess the new types of user/technology relations that are produced by the manufacture of disconnection and discuss what the implications are for resisting the attention economy. To do this, I analyse five technologies of disconnection utilising the walkthrough method (Light, 
Burgess, and Duguay 2016) and data from semi-structured interviews from the disconnectionists behind the technology. The research questions ask: what are the new modes of relations that the manufacture of disconnection produces, and how do these relations implicate resistance to the attention economy and culture of connectivity? My thesis builds upon research from disconnection scholars who relate disconnecting from the internet to the work of Michel Foucault (Guyard and Kaun 2018; Karppi 2018; Karppi and Nieborg 2020; Portwood-Stacer 2012b). Foucault's turn in the 1980 s to ethics of the self ("late Foucault") makes him an ideal theorist for a study on the manufacture of disconnection because of his consideration on how to resist the forces he believed were shaping society and individuals. Adopting a late Foucauldian perspective, this thesis identifies new relations of space and self that are produced by the manufacture of disconnection: a rehabilitative space; a sanctuary space; the fixable self, the intentional self and the available self. These spatial and self relations are digital architectures that enable inhabitants to resist dominant communicative norms or their own unconscious smartphone behaviours to transform their relationship to themselves, as well as seek refuge from certain surveillance activities that undergird the attention economy. Throughout my analysis I demonstrate that the manufacture of disconnection offers users an effective mode of lifestyle resistance to the attention economy but orients disconnection to be in service of productivity, wellbeing and gender norms that require users to subject themselves to additional self-governance methods. The thesis concludes that the manufacture of disconnection encourages new selfdisciplinary modes of living for users in the attention economy without dismantling the structures of the attention economy. 


\section{Table of contents}

Abstract

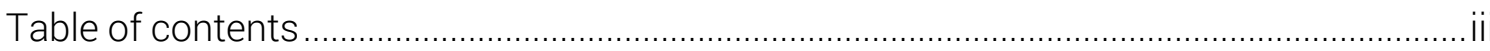

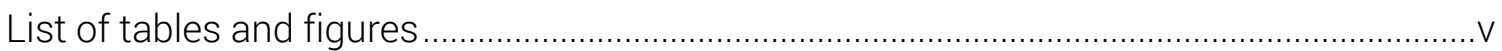

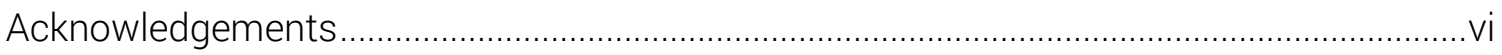

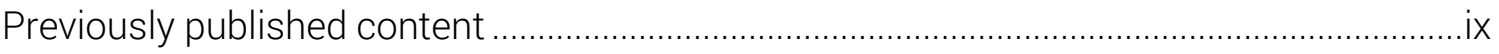

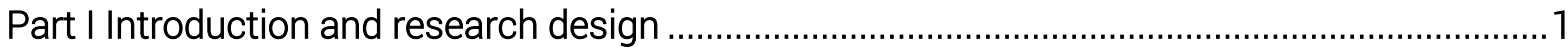

1. The disconnectionists and the paradoxes of their inventions ...............................................

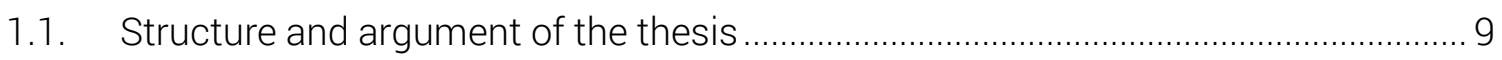

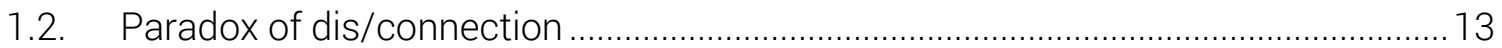

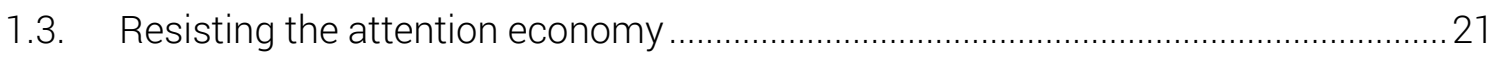

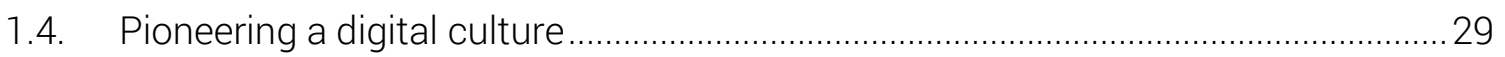

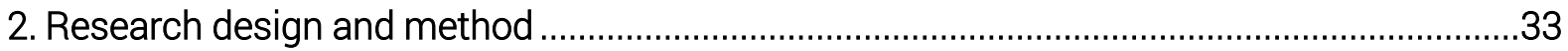

2.1. Research questions: reframing the paradox of dis/connection ................................34

2.2. Theory: late Foucault on power, technology and resistance ..................................... 36

2.3. Method: identifying and analysing technologies of disconnection...........................51

Part II The production of disconnected space ...........................................................................66

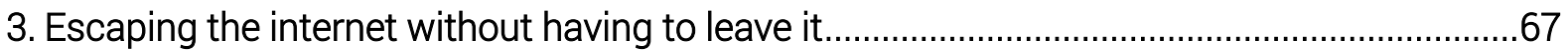

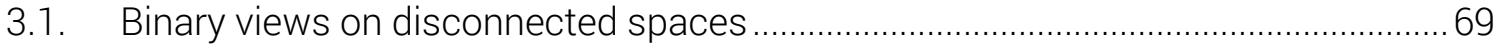

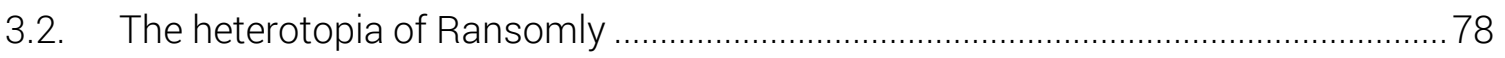

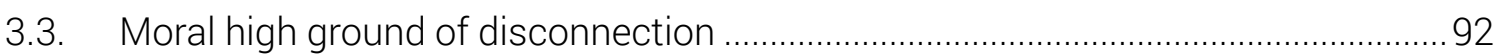

4. A digital sanctuary that provides immunity from surveillance ..............................................96

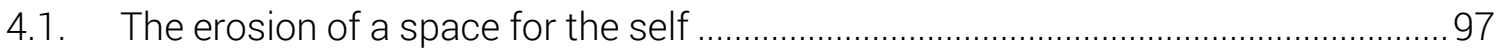

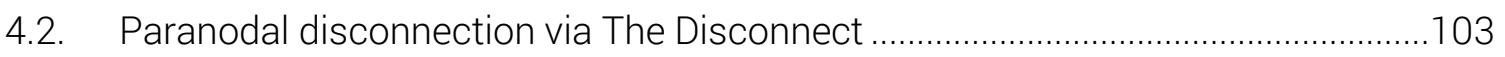

4.3. Immunity from surveillance ...................................................................................... 114

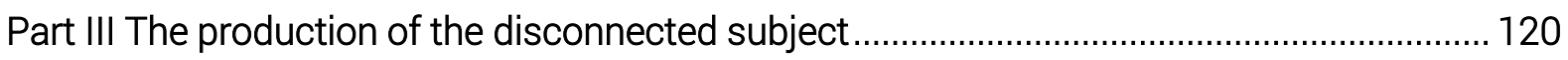

5. The fixable self and the ethical turn of persuasive technology ............................................ 121

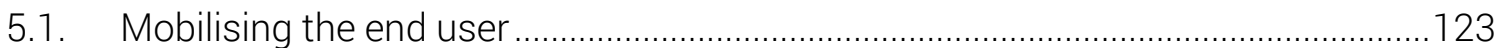

5.2. The therapeutic authority of Boundless Mind and Space ......................................129

5.3. The obfuscation of persuasive technology power ................................................ 138

6. The intentional self and the dropouts of Silicon Valley ...................................................... 142

6.1. Silicon Valley and intentional communities ........................................................ 144

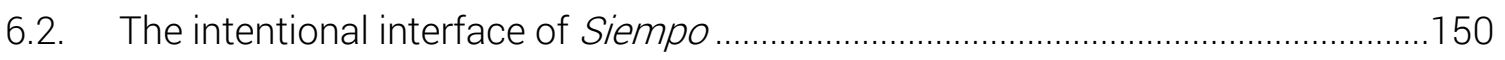

6.3. The authentic digital consumer ................................................................................ 164

7. The available self and the gendered inequities of disconnection........................................ 167

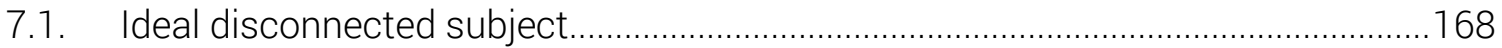

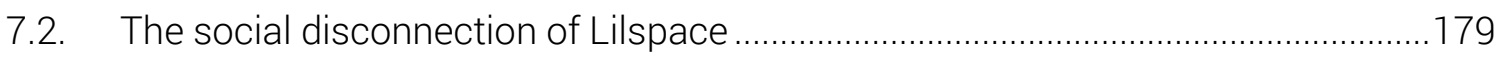




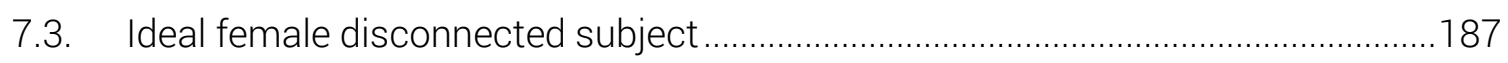

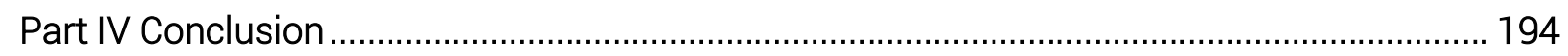

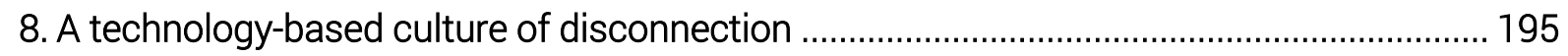

8.1. Disconnecting by inhabiting digital spaces and subject positions ........................ 197

8.2. The way forward and future research possibilities ................................................207

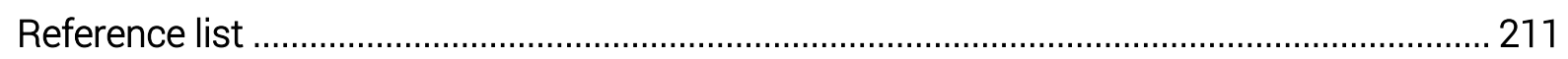

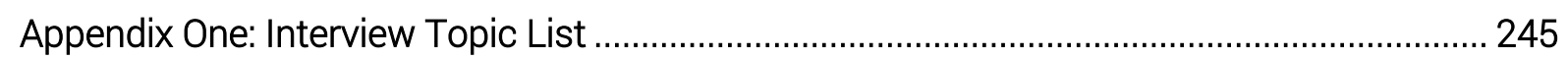




\section{List of tables and figures}

\section{Tables}

TABLE 2-A: INTERVIEWED DISCONNECTIONISTS AND THEIR TECHNOLOGIES OF DISCONNECTION. 54

TABLE 2-B: THE HOME SCREEN OF EACH OF THE FIVE TECHNOLOGIES OF DISCONNECTION

\section{Figures}

FIGURE 3-A: NO TECH-ZONE SIGNS. 67

FIGURE 3-B: CARTESIAN DISCONNECTION .73

Figure 3-C: PERMissions that enABLE RANSOMLY TO WORK 81

FIGURE 3-D: RANSOMLY BLOCK AND EXIT SCREEN .86

FIgURE 4-A: THE DisCONNECT MAGAZINE HOMEPAGE DESIGNED BY JACOB HALTON 107

FIGURE 4-B: THE DISCONNECT MAGAZINE AFTER THE USER DISCONNECTS FROM THE INTERNET 108

FIGURE 4-C: THE CONTENTS OF THE DISCONNECT MAGAZINE 109

FIGURE 4-D: AN ARTICLE ON THE DISCONNECT MAGAZINE.... 110

FIGURE 5-A: SPACE ONBOARDING PROCESS 130

FIGURE 5-B: SPACE HOMEPAGE AND PROCESS TO GET 'SPACE' 132

FIGURE 5-C: PROCESS TO GET A REPLACEMENT ICON 133

FIGURE 5-D: STIMULUS DEVALUATION IN ACTION 135

FiguRE 6-A: THE HOME SCREEN OF A SAMSUNG GALAXY 5 BEFORE AND AFTER INSTALLING SIEMPO 151

FIGURE 6-B: AN EXAMPLE OF THE INTENTIONAL INTERFACE AND CUSTOMISABLE BACKGROUND 152

FIGURE 6-C: A SCREENSHOT FROM THE SIEMPO WEBSITE 154

FIGURE 6-D: TOOLS AND 'FREQUENTLY USED APPS' OF THE SIEMPO INTERFACE 157

FIGURE 6-E: FLAGGED APPS OF SIEMPO 159

FIGURE 6-F: THE NOTIFICATION TEMPO FUNCTIONALITY..... 160

FIGURE 6-G: THE SIEMPO COVER SCREEN FUNCTIONALITY AT THREE, SIX, AND TEN-MINUTE INTERVALS. 163

FiguRE 7-A: SETTING A STATUS OF DISCONNECTION. 182

FIGURE 7-B: THE LILSPACE HOME SCREEN. 184

FIGURE 7-C: FURTHER STAGES OF THE LILSPACE UNPLUGGING PROCESS. 187

FIGURE 7-D: LILSPACE UNPLUGGING ACTIVITIES, CAUSES AND PLACES 190 


\section{Acknowledgements}

No one can undertake a PhD while being disconnected from other people.

I first want to thank my supervisors: Michael Daubs, Angi Buettner and Cherie Lacey. They helped me to develop rigorous arguments and see connections in my work that I could not. In particular, Michael provided the most meticulous feedback on draft manuscripts that was driven by an indefatigable determination to eliminate any vague pronoun from my writing. Angi had the foresight to insist that I ground my work in Foucault and really drove me to demonstrate the significance of this project. And Cherie provided invaluable insights in how I could use Foucault and turn my positionality and experience of disconnection into a strength of my analysis. I am so grateful for all of your mentorship and guidance over the last three years.

I was lucky enough to receive support from wider Te Herenga Waka-Victoria of University of Wellington (VUW) academics and staff. Kathleen Kuehn, Peter Thompson, Sally Riad and Trisha Dunleavy regularly offered encouraging advice along my PhD journey. Without knowing what he was getting himself into, Tim Corballis kept an eye on me during my first international academic conference in New Orleans, the highlight of which was attending a memorable live performance together at the famous Preservation Hall. Nayantara Appleton and Jayne Krisjanous provided me with the opportunity to test my ideas about disconnection via guest lectures and seminars, and thanks to Harry Ricketts I was able to stretch my writing muscle by reviewing books for the New Zealand Review of Books.

Early on in my PhD I turned my research in a wellbeing workshop for Mauri Ora Health and Student Academic Services at VUW to support students who struggle to focus because of social media. This could not have happened without the support from Jude West and Trish 
Knight who backed my vision from the get-go. I'm also thankful to fellow VUW PhDs Rebecca Galbraith and Kerry Palmer for helping me to set up and run a school seminar series for postgraduates to showcase their research, and the students who came along to support it. The seminar series was short but sweet.

One of the benefits of studying a relatively new discipline like disconnection studies is the open community of practitioners and scholars who make it up. I want to offer a big thank you to the technologists behind the manufacture of disconnection who took the time to speak with me in person or over Skype. I also cannot overemphasise how thrilling it was to meet and pick the brains of disconnection scholars such as Elija Cassidy, Aleena Chia, Kate Mannell, Ethan Plaut, Magdalena Kania Lundholm, Anne Kaun, Tero Karppi, and Ben Light at conferences or via Skype or Zoom. They have all inspired my research and it is a privilege to contribute to this exciting field of research.

I've made some academic friends and colleagues along the way, particularly when I attended a stimulating PhD summer school at the Digital Media Research Centre (DMRC) at the Queensland University of Technology in 2019. Jean Burgess and her team of researchers at the DMRC have created a vibrant research culture for PhD students to take their thesis to the next level and I especially want to thank Benjamin Nichol for pushing me to integrate an equity-based approach to my research on disconnection. More broadly, I really cherish the friendships I've made with fellow PhDs and early career researchers along the way. Will Abbiss and I broke down every Game of Thrones or Black Mirror episode in our office, perhaps to the annoyance of anyone who could overhear us, and I relished rambling about the PhD life with Ehsan Khanmohammadi Hazaveh along Wellington bush trails. 
I could not have completed a PhD without the encouragement from my close friends and family. My friends provided support along the tumultuous journey of doctoral research whether it was across a tennis court, or during book club, a trek and a birding-based adventure in Wellington. From a young age, my sister Emilie modelled a love of reading and during my PhD her bookstore happily supplied me with books at heavy discounts that would put Book Depository out of business. My parents encouraged me to pursue my ambitions and become the research nerd I was hesitant to develop into. My Mum is the most curious and creative thinker that I know, and an inspiration to push disciplinary boundaries and ask challenging questions. I'm sorry Mum (but not really sorry (-)) that I got to the PhD finish line just before you. And to my dearest Hannah, who I never want to disconnect from, we have done it! Through many tears, grit and determination, we returned to university at the same time and came out even stronger together. The grey hairs are even worth it. Your love, intellectual rigour (no one can match your wordsmith skills) and support has undoubtedly made me a better student, partner and person.

Lastly, I dedicate this thesis to my father and grandfather who passed away during my PhD. Without the confidence, knowledge and work ethic they passed onto me, none of this would have been possible. I love you both and hope to do you proud. 


\section{Previously published content}

Portions of this thesis have been published as:

Beattie, A. (2019). Immunity from surveillance: a magazine that forces readers to disconnect from the internet, Spectator. University of Southern California 39(2), pp. 8-15. 
Part I

Introduction and research design 


\section{The disconnectionists and the paradoxes of their inventions}

Smartphones are useful tools...but they're addictive. Pull-to-refresh is addictive. Twitter is addictive. These are not good things. When I was working on them, it was not something I was mature enough to think about. I'm not saying I'm mature now, but I'm a little bit more mature, and I regret the downsides.

Loren Brichter (qtd in Lewis 2017).

In Silicon Valley, the world's most famous site of technological innovation, technology professionals are rejecting their own inventions and disconnecting from the internet. According to reports, some programmers believe their designs and algorithms "hijack" people's brains (Lewis 2017) and executives are sending their children to technologyfree schools (Jenkin 2015). Loren Brichter is the creator of the pull-to-refresh feature, a common design functionality to smartphone applications that enables the user to refresh the contents of the screen via a touchscreen gesture. He is also one of many technologists and designers who feel responsible for tethering millions of people to their smart devices. These designers are not disillusioned by digital technology per se, but rather by the ideological and socio-economic system underpinning digital technology. This system is the 'attention economy', in which media companies, advertisers and technology platforms treat end user attention as a finite commodity to compete for (Crogan and Kinsley 2012). At an individual level, the attention economy incentivises workers like Brichter to create habitual technological experiences that glue users to their screens and, at an industry level, reorganises the internet into a persuasive advertising platform (Wu 2016).

By disconnecting from their own inventions and the internet, technologists such as Brichter have become 'disconnectionists' (Jurgenson 2013), opponents of the culture of 
constant connection they helped create. Nathan Jurgenson (2013) introduced the concept of disconnectionists to criticise late 2000s and early 2010s advocates of disconnection such as technology scholar and psychologist Sherry Turkle (2013) and technology writer Paul Miller (2013). According to Jurgenson (2013), these thinkers share a view that excessive internet connectivity or social media participation threatens a genuine representation of the self: "the disconnectionists see the Internet as having normalised, perhaps even enforced, an unprecedented repression of the authentic self in favour of calculated avatar performance". Therefore, Turkle and Miller are disconnectionists because their response to excessive screen time is to practice disconnection, or call for others to disconnect from the internet to protect the self. Jurgenson (2013) considers the disconnectionist perspective to be overblown considering that debates about performativity and authentic self-representation have long predated the emergence of social media or digital media technology (for example, see Goffman 1969). He contends that "the more we argue that digital connection threatens the self, the more durable the concept of the self becomes." What can be inferred from Jurgenson is that a disconnectionist seeks to disconnect because of a fixed or conservative view of the self and an interest to suppress any online presentation of themselves that they believe to be inauthentic.

Since Jurgenson introduced the term disconnectionist in 2013, methods and practices of disconnection have become increasingly popular and diverse. Disconnecting from the internet has become a health and tourism activity and is undertaken in the form of digital detox retreats (summer camps for adults where networked technologies are banned; Sutton 2020), 'dopamine fasts' where people abstain from pleasure-inducing activities such as social media scrolling (Way 2019), Wi-Fi free cafés and hotels, and National Unplugging Days. It has become fashionable in some urban parts of the world, such as New York, to 
deliberately choose a feature phone (a mobile phone that lacks the advanced functionality of a smartphone) over a smartphone device (Portwood-Stacer 2012c). In 2018, \#deleteFacebook trended on Twitter following allegations that Facebook was complicit in the Cambridge Analytica data hacking scandal, suggesting disconnection is a practice to mitigate potential privacy concerns (Brown 2020; Laterza 2018). In France, disconnection has become a right by being enshrined in labour law. French workers in organisations with over 50 staff have a 'right to disconnect' to prevent work burnout and address the work/leisure slippages that can occur during flexible and remote working (Hesselberth 2017). Even Apple (2018) and Google (2018) have become disconnectionists by respectively offering Screen Time and Digital Wellbeing as tools for users to disconnect from their devices more easily. Both tools offer information to the user about time spent on the device and functionalities that can nudge the user offline. These examples indicate that disconnecting from the internet is a political, cultural, socioeconomic and lifestyle practice that is being realised in an increasing number of ways. In contrast to the view that disconnection is a narrow-minded exercise to restore the self to a more authentic offline existence (Jurgenson 2013), disconnectionists appear to be more broadly motivated to disconnect because of concerns around issues such as data privacy, surveillance, work/leisure slippages and the perceived harms of connectivity on the self.

However, not many people can be a disconnectionist. Although methods of disconnection appear to be expanding, the choice to actively disconnect from the internet is not available to many groups of people. To choose to disconnect and oppose the idea of constant connectivity presupposes reliable access to the internet and secure employment. Nearly 40 percent of the world's population still lack regular access to the internet (Internet World Stats 2020). Many workers in the digital economy, such as Uber drivers and Facebook 
content moderators, do not have the luxury of disconnecting from their devices or the agency to choose what to disconnect from. Brichter and the Silicon Valley disconnectionists enjoy immense privileges that raises the question of who can disconnect from the internet and resist the attention economy, and on what terms.

Another advantage that the Silicon Valley disconnectionists benefit from is their technological prowess and insight. They have the technological expertise to turn disconnecting into an inventive practice. Not only are these disconnectionists disconnecting from their own creations but, in true Silicon Valley style, are also inventing new technological-based ways to do so. Creating technology-based ways to disconnect from the internet is itself not particularly unusual for Silicon Valley. According to Evgeny Morozov (2014) there is a dominant mindset in the technology industry called 'technological solutionism', where Silicon Valley technologists, entrepreneurs and executives uncritically position technology as the default solution to societal issues. Following this logic, the disconnectionists appear to be the latest group of technologists who are transforming another aspect of life into a technological solution. What makes the Silicon Valley disconnectionists unique and difficult to place is their ambivalent relationship to connectivity and technology. Put simply, the disconnectionists invent technology-based ways to disconnect to resist prevailing technologies that they often have a hand in creating. We therefore cannot call the disconnectionists 'technophobes', as they seek to address the problem of technological overload and screen addiction with a technological solution. Neither are the disconnectionists neo-Luddites, as their jobs have not been displaced by 
emerging technologies. ${ }^{1}$ If anything, the disconnectionists are part of the professional class who are responsible for displacing other workers via their technological inventions. Through new technological practices and solutions, the Silicon Valley disconnectionists are rejecting a culture of constant connection while simultaneously pushing forward a new technologybased culture of disconnection. Taken together, these disconnectionists are manufacturing disconnection.

This thesis critically examines the technological and cultural aspects of Silicon Valley-driven disconnection to understand what it means to disconnect in the digital age. My investigation begins with a brief discussion of what I mean by manufacturing disconnection. On one level, the term manufacture simply means to make, suggesting the manufacture of disconnection is a technical account about technologies, specifications and functionalities ("technologies of disconnection") that enable disconnecting from the internet. These types of technologies of disconnection include smart device applications (apps), internet jammers, and luxury feature phones that enable a particular type of disengagement from the internet. Some of these technologies are obscure digital media experiments that operate at the fringe of digital culture, while others are more popular applications that have received significant mainstream press or been sold and integrated into larger platforms. As a whole, these technologies use a mix of hardware, software protocols, behavioural science techniques and design features that operationalise disconnecting into a technical process and solution that is largely understudied. While scholars have applied behavioural science theory to assess technologies of disconnection (Lyngs et al. 2020; Lyngs et al. 2019) or discussed them in

\footnotetext{
${ }^{1}$ See Lachney and Dotson (2018) for an overview of the term Luddite. The authors argue that normative usages of Luddite to infer anti-innovation are a far cry from the original use of the term, which was to capture a group of anti-automation textile workers (allegedly lead by Ned Ludd, hence the term 'Luddite') who protested automation by breaking weaving machines.
} 
relation to existing regimes of power and modes of resistance (Gregg 2018; Guyard and Kaun 2018; Karppi 2011; Plaut 2015; Thorén et al. 2017), none have sought to disassemble the components of these technologies and critically examine them in greater detail. In this thesis, I deconstruct the design of these technologies to understand how software, behavioural science and other technical practices configure and transform disconnection into something active and knowable. While I do not suggest a type of technological determinism that implies that software and behavioural science are predominantly shaping notions of disconnection, we do not know how these scientific and technical practices are influencing the way people disconnect from the internet, warranting further attention.

Another interpretation of manufacture is to fabricate. Taken this way, the manufacture of disconnection is less about any actual separation from the internet, and more of an imitation of what we might expect disconnection to be. In the most famous example of media-driven fabrication, Edward S. Herman and Noam Chomsky (1988) argue that the American mass media in the 1960s and 70s manufactured audience consent by deploying various filters to subtly shape news coverage while retaining a façade of news objectivity. Herman and Chomsky's overarching point is that the use of news filters effectively manipulated audiences to accept news propaganda. In the manufacture of disconnection, the equivalent sleight of hand is that no literal disconnection, meaning severing of connections from the internet or physical distancing from digital media technology often occurs, with users seemingly 'disconnecting' while remaining connected to their devices. The fabrication is that the user is not actually disconnected from the internet, but rather from certain features of the internet, or aspects of themselves. Many of the disconnectionists that I spoke to during this research view the term disconnection as a pejorative, preferring to associate their technologies or businesses with wellbeing or self-care ideals that are popular in the Silicon 
Valley (English-Lueck 2010). I explore how these wellness and self-care ideals act as filters by encouraging new subject positions, relations and mediated degrees of separation that users of technologies of disconnection have with the internet and themselves. While this type of disconnection is not necessarily overtly deceptive, some features are designed to encourage users to manipulate themselves to avoid future behaviours that the user indicates they wish to prevent. Moreover, there are cultural practices and ideological components embedded in technologies of disconnection that shape what it means to disconnect, and therefore should be discussed in greater detail.

I adopt this double meaning of manufacture. Not only do I investigate how disconnection is made into a technology, I also analyse how disconnection is fabricated via the reorganisation of relations with technology instead of severing relations altogether. Interpreting manufacture as both an act of technological construction and social fabrication, provides insight into what the disconnectionists want to disconnect from; what practices they believe warrant sufficient disengagement from the attention economy, or any other aspects of technology they see as problematic. In other words, the manufacture of disconnection not only reveals how we are encouraged to disconnect with technology, but also reminds us to consider what we are disconnecting from, why we disconnect and who ultimately benefits from the disconnection. We get an opportunity to discuss the consequences when disconnecting becomes more about a new relationship with the self and technology than an express act of distancing oneself from technology. Altogether, the manufacture of disconnection presents an ideal entry point to understand how a loosely affiliated group of technologists who share concerns about constant connectivity and the attention economy are influencing the design of our mediated environment, creating new 
types of mediated space and self, and more broadly, what it means to disconnect in the digital age.

\subsection{Structure and argument of the thesis}

The structure and argument of this thesis is as follows. Chapters one and two comprise Part I of this thesis. In the rest of chapter one, I address the contradiction of having to connect to disconnect that the manufacture of disconnection supposedly raises, the issues at stake, and the potential insights that this topic offers to digital culture and media studies. Chapter two responds to the contradictions and issues by presenting my approach to studying the manufacture of disconnection. I outline my research questions, which aim to assess the viability of the manufacture of disconnection as a means to resist the attention economy. I then explain how my interpretation of the manufacture of disconnection as reorganising users' relations with technology is influenced by the eminent relational thinker Michel Foucault. I argue that Foucault offers a suitable theoretical framework to study the manufacture of disconnection given his relational approach to studying power, resistance and technology. In particular, Foucault's ideas on utilising the same forces to resist prevailing forces provides a framework to address the dichotomous issues that the manufacture of disconnection raises and untangle the contradictory relationship that the disconnectionists have with technology. Following this, I discuss how I use the walkthrough method and data from semi-structured interviews with the disconnectionists to answer these questions. These methods provide me with comprehensive insight into how disconnection is imagined, made and realised into new relations with the internet.

Parts II and III offer my analysis as to what the manufacture of disconnection entails. In Part II, I argue that the manufacture of disconnection produces disconnected space to 
resist the attention economy. In chapter three, I discuss a new type of mediated rehabilitative space that allows a user to escape the internet without having to retreat from it. I argue that this type of rehabilitative space contrasts with dominant scholarly and popular discussions about disconnection. I outline how disconnection has commonly been discussed by media studies researchers in terms of spatial extremities: as either an information-poor dystopia that the developed world has progressed past, or as a distraction-free utopia that only the privileged can access. In contrast to these spatial extremities is Ransomly, an internetjamming beacon that works with smartphones to create an internet dead zone in a location of one's choice. Drawing upon Foucault's concept of heterotopia, or an 'other space' (Foucault 1986), I contend that Ransomly offers a type of disconnected space that is neither utopian nor dystopian, but merely different by offering inhabitants the chance to resist dominant communicative norms and behaviours that have allegedly been driven by social media and ubiquitous internet access. However, I suggest that Ransomly also frames a disconnected space as the moral high ground, modelling appropriate smartphone use to convince users that occupying a disconnected space is worth their time.

Not all disconnected spaces claim the moral high ground or conflate disconnecting with wellbeing ideals. In chapter four, I analyse The Disconnect, a digital offline magazine that forces its readers to disconnect their internet connection in order to unlock the media content of the magazine. The Disconnect is a radical departure from the way online content is published in the attention economy. I argue that standard publication practices in the attention economy such as banner advertisements and data-driven content analytics have eroded any space for users to engage with news or content on their own terms. In contrast, The Disconnect shifts the reader off the network, which has the effect of providing shelter from surveillance and the logics of the attention economy. Connecting Foucauldian 
resistance to the spatial concept of paranodality, or 'beside the network' (Mejias 2013), I argue The Disconnect provides a type of sanctuary for the self by providing readers temporary immunity from certain commercial surveillance practices. Yet the separation between the user and the attention economy caused by The Disconnect reduces the digital offline magazine to an artistic experiment instead of a viable alternate model to delivering content in the attention economy.

In Part III, my analysis shifts to how the manufacture of disconnection produces disconnective subject positions, or new relations of self for the user. I observe how technologies of disconnection encourage users to disconnect from an aspect of themselves and view their online behaviours in a specific way, allowing users to develop more critical and sensitive understandings of their online habits and behaviours. The first relation I observe is the fixable self, which I discuss in chapter five by exploring a habit-breaking smartphone application called Space. Space leverages insights from behavioural science to prevent users from subconsciously opening any flagged applications. This functionality encourages users to view unconscious scrolling as an undesirable habit that is fixable. I argue that the fixable self is driven by the application of behavioural science in app design and an ethical turn by app designers towards their practice. Noting how widely behavioural science is applied in app design, I contend that the focus on the fixable self valorises the expertise of app designers while obscuring their role in tethering users to their devices in the first place.

In chapter six, I identify another behaviour-based relation that encourages a selfmanagerial digital lifestyle that I call the intentiona/self. What separates the intentional self from the fixable self is the cultural context. I argue that the intentional self draws upon a Silicon Valley counterculture from the 1960s and 70s that emphasised self-transformation and goal-orientated living. The ethos of historical Silicon Valley countercultures like New 
Communalism and intentional communities lives on in a smartphone app called Siempo, which reorganises the user's home screen and forces the user to perpetually self-manage their digital life and to set day-to-day goals. I demonstrate how Siempo users are guided to act intentionally at all times on their smartphone, which positions resistance to the attention economy as a lifestyle practice that focuses on limiting unintentional digital consumerist activities.

The third and final relation is the available self. In chapter seven, I present the available self as emerging in response to the issue of who can resist or disconnect from the attention economy, and on what terms. I argue that Western society has an underexamined dominant model of disconnection that is produced by hegemonic masculinity discourses. I identify the ideal disconnected subject as one who is male and unencumbered and can cut their social ties at whim, something that is often not available to women and professionals who are socially burdened with relational obligations, such as parenting or managing social connections. An app that appears to recognise the inequities of disconnection by making disconnection more relationship sensitive is Lilspace. However, as the analysis of the app will show, Lilspace encourages users to disconnect in a way that does not forestall the gendered expectations of their social role. Instead, Lilspace users are encouraged to socialise their disconnection and become the 'available self' during the process of disconnection. The available self does not dissipate gender norms but reinforces them, offering a type of resistance to the attention economy that is highly gendered.

In Part IV, I bring together all five relations as digital architectures that constitute a technology-driven culture of disconnection to resist routine living in the attention economy. Drawing on Apple's Screen Time and Google's Digital Wellbeing as evidence of two of the largest technology companies integrating technologies of disconnection into their platform, I 
summarise what the manufacture of disconnection actually resists and discuss the implications of this supposed resistance for users. I conclude that the manufacture of disconnection resists distractions and communicative norms, such as the burden of constant availability that is perceived to be exacerbated by the attention economy and offers new governance techniques for users to self-regulate their smartphone behaviours. I argue this type of resistance is a moralised and, at times, gendered practice that frames disconnecting as an alternative self-disciplinary lifestyle for individual users to undertake. Moreover, I contend that new modes of living in the attention economy are appealing to technology platforms like Apple and Google, as they indicate another category of functionalities and products for platforms to govern the smartphone behaviours of users in new ways. To this end, I argue that the manufacture of disconnection extends the power of technologists in the attention economy when locating the disconnection as between the user and their behaviours, as opposed to disconnecting the user from the logics of the attention economy. I close the thesis by discussing opportunities for future research on the manufacture of disconnection.

In the remainder of this introduction, I further contextualise the manufacture of disconnection by providing an account of the contradictions and key issues that are at stake.

\subsection{Paradox of dis/connection}

At the centre of the manufacture of disconnection is a giant contradiction. The paradox is that digital technology is being used to resist prevailing digital technology. I was made well aware of this contradiction throughout my PhD when explaining my research to friends, family and colleagues. I heard countless variations of: "Why would someone use their phone to disconnect from their phone? What a paradox!" Given the frequency of this response 
to my dreaded thesis explanation, it is perhaps not surprising that this paradox is at the forefront of disconnection studies. According to scholars who have recently surveyed disconnection literature, enquiring into the technological side of disconnection is problematic because it risks normalising the technological (Kuntsman and Miyake 2019) or reinforcing a paradox of having to connect to disconnect ("dis/connection") (Hesselberth 2017). The authors in these studies criticise researchers, policy makers, and practitioners for insufficiently distinguishing disconnection from technology or connectivity. An example that Hesselberth (2017) points to is a study by Wyatt, Thomas, and Terranova (2002) on categories of non-users. Hesselberth criticises the study for utilising empirical methods to conceptualise types of non-use that are typically invisible or passive and can, at best, only be partially captured by empirical methods. Hesselberth argues that the study categorises disconnection in a way that risks instrumentalising insights about disconnecting to serve connectivity. Following Light and Cassidy's (2014) observation that disconnection offers a type of 'socio-economic lubricant' to social media platforms like Facebook by ensuring continued user participation on the platform, Hesselberth's concern is that businesses such as Facebook can use information about types of non-use to connect users in alternative and invisible ways, which would in turn enable Facebook to extend the culture of connectivity and subvert the point of disconnection in the first place.

The paradox of dis/connection is a provocative argument that has implications for disconnection research. I believe the most compelling point Hesselberth makes has to do with the transformative potential of disconnection, where disconnecting is understood as a mode of disengagement or an action-based verb of breaking away from something. The potential of disconnecting, according to Hesselberth, is not in the rediscovery of age-old themes such as productivity or solitude, but in the possibility of creating an outside to 
whatever is being disconnected from. Quoting Ned Rossiter $(2004,21)$, Hesselberth $(2017$, 2007) argues: "[disconnecting] is not so much about the refusal or dislike of 'technology', but rather 'operates as an affirmative force that holds the capacity for transformation'". In other words, disconnection is not revelatory because it reminds us of the benefits of time spent alone, but because of the action potential of disconnection itself: the disengagement from something. By breaking a connection to something, such as a distracting website, a commercial practice, or a smartphone entirely, the act of disconnection can constitute an outside to whatever was disconnected from and create a transformative experience for the disconnecting user.

Hesselberth's concern is that disconnection scholars have largely neglected this aspect of disconnection and therefore, as it stands, the potential of disconnection to resist connective culture and the underlying regimes of power are understudied. In addition, Hesselberth is herself sceptical about the transformative power of disconnection. In a later study, Hesselberth (2019) analyses the tiny-home movement in the Netherlands as an example of people attempting to disconnect from the capitalist property market by living offthe-grid. Hesselberth $(2019,122)$ acknowledges that tiny homes offer potential owners the means to resist "growth-based economics" by having less financial debt and relative autonomy from infrastructures such as electricity and municipal water supplies through onsite renewable energy sources. Yet tiny homeowners still live within a property market (albeit a different one), pay rates, and are subjected to different landowner regulations. Hesselberth's point is that disconnecting from one regime of power often requires subjection to another one. As Lorey $(2006,136)$ argues, "agency emerges in the paradoxical movement between subjugation and empowerment, coercion and freedom." The paradox of dis/connection in the case of tiny homes is that the potential to live off-grid and escape 
capitalism comes with a big caveat: exiting one regime requires living under another. In a study where participants were forced to disconnect from Facebook, Baym, Wagman, and Persaud $(2020,9)$ similarly conclude that disconnecting from Facebook will not transform Facebook, writing: "Consciousness and mindfulness, no matter how diligently practiced, cannot save our landscape from the vegetation cover of Facebook. Disconnective practices may help people find balance in the trap, but it cannot set them free." The inference from these scholars is that disconnecting on the terms that it is currently understood is an ineffective practice to resisting power.

Until scholarly conceptualisations of disconnection are sufficiently divorced from elements of connectivity, Hesselberth does not believe that a robust culture of disconnectivity (with associated rights and norms to refuse or opt-out from media) can emerge. The type of disconnection that Hesselberth (2017) believes many users of digital technologies pine for is a 'normative gesture' towards disconnectivity; in which people withdraw from internet and mobile networks to achieve unmediated experiences. Hesselberth infers that mediated, technologised or any mode of disconnection that reinforces the paradox of dis/connection is incapable of "weaken[ing] the grip these technologies of mass connectivity have on us" (Hesselberth 2017, 2005) and subsequently betray the potential of disconnection to resist connective culture.

The paradox of dis/connection appears to address the manufacture of disconnection. If we were to accept Hesselberth's reasoning, a premature conclusion of this thesis would be that the whole idea of manufactured disconnection conflates connection with disconnection, creating a cheapened version of disconnection for the user that requires an active connection to the internet, with the user's dependence to technology only deepening. However, I do not simply adopt Hesselberth's arguments. Unlike Hesselberth, I am not interested in making any 
normative claims as to what disconnection is, nor what a culture of disconnectivity should look like. Similar to Karppi $(2018,3)$, I aim to avoid "the essentialist question of what disconnection is", focusing instead on how the Silicon Valley disconnectionists interpret the practice of disconnecting, how they do it, and where the actual disconnection occurs (if at all). Most significantly, however, I do not agree with everything that the paradox of dis/connection suggests, which I discuss in the next section.

\subsubsection{Binary framing of disconnection}

My first issue is that the paradox of dis/connection unnecessarily positions disconnection in an antagonistic relationship to connection. I do not believe that disconnection is best conceived as being at odds with connection, because a disconnection cannot occur without a connection. The term dis-connection is a negation of connection, with any disconnection needing a connection to break in the first place. The framing of dis/connectivity as a paradox is therefore misleading, as it structures the communicative states of dis/connection, non/use and on/offline into binary and oppositional terms. Jurgenson (2012) identifies the categorisation of on/offline to be evidence of digital dualism-a Cartesian perspective that assumes physical and digital space are experienced distinctly. The insistence on a rigid distinction between physical and digital space frames disconnection as limited to physical space, which lends itself to an over-generalised and technophobic perspective that all digital or mediated practices are unhealthy or only represent connective behaviour (discussed further in chapter three). As Light (2014) suggests, users of digital media technologies often perform practices of disconnection while remaining connected to the internet, such as unfollowing someone on Facebook or blocking someone on Twitter. These examples suggest that even in the short history of social media, people have been disconnecting with (in contrast to against) technology. Light $(2014,4)$ 
offers a theory of disconnective practice as "the potential modes of human and non-human disengagement with the connective attempts made possible with social networking sites". By emphasising the opportunities to disconnect with technology, Light avoids discussing disconnection as an idealised unmediated communicative state in favour of observing where the disconnection from social media actually occurs and the variety of ways users undertake disconnection while still connected to the internet. Natale and Treré $(2020,630)$ similarly observe hacking and anonymous browsing practices on the internet as examples of disconnecting while using technology, stressing that disconnection can be an act of engagement and practice to "deepen and problematize our engagement with the digital".

By relying on binary notions, the paradox of dis/connection presents a false dichotomy that overlooks the multitudes of disconnections that occur in digital culture every day. To demonstrate the breadth in which the concept of disconnection can illuminate the digital and the technology industry, Karppi (2018) argues that Facebook views disconnection as a threat to its business. Following Gilles Deleuze and Felix Guattari's (1990) discussion of immanence, Karppi interprets disconnection as a multifaceted and interactive agential force as opposed to a singular communicative state. He demonstrates that disconnection is already shaping connective culture, pointing to Facebook changing its policies and protocols concerning user account deactivation and deletion in the hope of convincing users to never leave the platform. The practices that Facebook deploys when users choose to delete their account include a mandatory stand-down period of 90 days and the deployment of affective content (e.g., images of close friends accompanied with the message you "will be missed") to prevent permanent account deletion.

Binary notions of dis/connection also only provide limited conditions in which the transformative potential of disconnection can occur. While I believe it is useful to emphasise 
disconnection as a mode of disengagement or an action-based verb of breaking away from something, this does not mean breaking away from everything or al/connections. No person or user of technology lives in a vacuum or is totally disconnected from all things, human or non-human. I instead interpret the manufacture of disconnection as mapping onto a spectrum of dis/connectivity, as opposed to a rigid paradox that implies the only type of disconnection worth enquiring about is the unmediated variety. Having to connect to disconnect becomes less of a concern because disconnections have always existed in relation to connections. When viewing disconnection this way, an important shift of enquiry occurs concerning the transformative potential of disconnection. A dis/connection could occur within the user or self, or signify a change of relations between the user and an application or platform. In short, bypassing the binary framing of dis/connection allows for an expansion of what I mean by the transformative potential of disconnection.

\subsubsection{Black boxing technology}

The paradox of dis/connection also functions as an impasse, making research about technologically enabled or manufactured disconnection more difficult. The paradox implies that to study the manufacture of disconnection risks advocating for a type of digital positivism, whereby having to connect to disconnect is assumed to wholly capture or dominate the experience of disconnection. These are the concerns that Kuntsman and Miyake (2019) raise in a critical analysis of disconnection literature, warning scholars to avoid naturalising technology in relation to disconnection. Pointing towards smart homes and technologies of disconnection as examples of the delegation of human agency to more technology, Kuntsman and Miyake build upon Morozov's (2014) concept of technological solutionism, to argue that disconnection discourse reinforces 'digital solutionism' and uncritically foregrounds media when considering how to escape media. By criticising the 
connectivisation or technologisation of disconnection, the arguments offered by Hesselberth (2017) and Kuntsman and Miyake (2019) are similar to 20 th century philosophers of technology, for whom the main issue was technology itself (Borgmann 1984; Feenberg 1991; Heidegger 1977). These philosophers saw technology as a monolith, contending many $20^{\text {th }}$ century societal issues were due to the pervasiveness of technology-based thinking in interpreting reality, politics and social issues. By viewing technology in equally macro and abstract terms, Hesselberth (2017) and Kuntsman and Miyake (2019) risk backing disconnection studies into a corner, whereby the only path forward is for researchers to crudely advocate for less technology or fewer connections. Without deconstructing the mechanisms of platforms that enable or prevent disconnection, these scholars risk a position Geert Lovink $(2019,59)$ calls 'platform nihilism', where researchers and users alike become "trapped in smoothness, skimming a surface littered with impressions and notifications" and are resigned to accept technologies of disconnection on the terms that they are presented to them.

Perhaps disconnection research can get beneath the surface of technologies of disconnection, and not black box it altogether. A study on the manufacture of disconnection does not have to naturalise technology but can denaturalise technology and the power relations beneath it. In other words, a deconstruction of manufactured disconnection should render visible the underlying structures of disconnection in our contemporary media environment and demystify any technological aspect of disconnection that we currently do not fully understand. Aside from observations that social networking platforms anticipate user disconnection to try to prevent it from happening (Karppi 2018; Lanier 2018) and that utilising hardware and software can make disconnection easier (Plaut 2014, 2015), current scholarship is largely silent regarding what components of technology are being used to 
structure, produce, and control end-user disconnection. As such, we do not know much about the potential of technologies to enable disconnection. Only by highlighting specific aspects of technology that configure and operationalise disconnection can a deeper understanding of the relationship between technology and disconnection emerge. In turn, these insights can contribute towards a robust and critical understanding of the technology-driven culture of disconnection. However, before we can examine how disconnection is made through mechanisms of power and control, we must explore the issues at stake in the attention economy.

\subsection{Resisting the attention economy}

Media scholars have examined the attention economy and discussed related modes of resistance, revealing important insights that are relevant to the disconnectionists and the manufacture of disconnection. The first theme in existing literature is why the attention economy is worth resisting. Research suggests that the attention economy is worth resisting because of how the attention economy structures the internet (Citton 2017; Wu 2016). The core logic of the attention economy is that for any business that depends on advertising revenue or subscriptions, the longer they can hold the attention of a user, the more revenue opportunities that business will have due to the user seeing more adverts, generating more data, and being more willing to subscribe to the service of the business (Wu 2016). Attention economy logic has long informed how media technologies have been designed, all the way back to late $19^{\text {th }}$ century newspaper editors who sensationalised their front-page stories to sell the whole newspaper (Crogan and Kinsley 2012; Wu 2016). With over three billion monthly active users between them, the current leaders of the attention economy are Facebook and Google (Wu 2016). Over the last decade, these technology conglomerates have dominated the 
attention or advertising market (Wu 2016), hiring the behavioural scientists and technologists behind the design of slot gambling machines in casinos (see Schüll 2012) to optimise the appeal of their social media products (Leslie 2016). These behavioural scientists and technologists have pioneered functionalities such as the bottomless social media news feed to encourage endless scrolling and red coloured notifications to associate social media communications with feelings of urgency (Harris 2016).

Research also indicates that the disconnectionists manufacture disconnection to resist being subjected to the logic of the attention economy. The perceived cost for users in the attention economy is a reduction of their agency. The stereotypical picture that captures this concern is people in public spaces glued to their devices. To the disconnectionists, this image is evidence of a widespread lack of willpower with user behaviour reduced to lab rats that have been conditioned by the designers of the technologies. Such concerns are reminiscent of media effects theory, which has a long and messy history investigating the influence of media on the attitudes and perception of audiences (Neuman 2016). Whether the attention economy and designers unduly influence the behaviours of users is equally divisive and difficult to prove. ${ }^{2}$ The disconnectionists refer to popular psychology and technology books (see Alter 2017; Carr 2011; Soojung-Kim Pang 2013; Twenge 2017) that warn about the possible effects of behavioural design on susceptible brains. Former Google designer and ethicist Tristan Harris suggests design features such as Snapchat's Snapstreak exploit the burden to socially reciprocate and be constantly available (Bosker 2016). These communicative norms have been linked to increased rates of anxiety, depression and poor

\footnotetext{
${ }^{2}$ There are multiple industry and academic discourses that frame the persuasiveness of behavioural design as an ethical issue. For industry discussion of unduly persuasive design, see Harry Brignall (n.d.) on 'dark patterns'. For academic analyses see Thaler and Sunstein (2009) on 'nudges'. In chapter five I interrogate the ethical responsibilities of behavioural designers and technologists in detail.
} 
sleep (Büchi, Festic, and Latzer 2019; Ling 2016; Reinecke et al. 2017) and losses in focus and productivity (Duke and Montag 2017; Mark, Gudith, and Klocke 2008). Yet any causal link between the use of digital media technologies and mental ill health is unproven and highly contentious (Orben, Dienlin, and Przybylski 2019), perhaps in part due to the multiplicities with how digital media technologies can be used.

Empirical evidence of the effects of the attention economy on users may be beside the point. Measuring the effects of actual online advertising on users beyond what they click on is notoriously tricky because of the crudeness of measurement metrics (Lewis, Rao, and Reiley 2015). Yet advertising remains a multi-billion-dollar industry, suggesting that empirical evidence to prove the effects of the attention economy on consumers is largely irrelevant. The attention economy certainly does not require evidence to function. Judy Wajcman (2015) makes a similar point in her research on the subjective experience of time for smartphone and internet users. The question of whether smartphone users objectivity or empirically have less or more time is not the primary point of her enquiry. What is just as revealing to Wajcman (2015) is that smartphone users report having subjective feelings and feel internal pressure about having less time. For the purpose of this thesis, the important factor is that there is a sufficient perception of the effects of the attention economy.

There is a strong perception amongst some scholars and practitioners that the attention economy is harming individual users and society. Harris (2016) contends Facebook and Google compete in a race to the "bottom of the brain stem", exploiting en masse the compulsive and irrational side of human behaviour. Philosopher and virtual reality pioneer Jaron Lanier (2018) rearticulates the attention economy as the B.U.M.M.E.R industry, standing for Behaviours of Users Modified and Made into an Empire for Rent. His concerns are that the behavioural designs created by the likes of Facebook and Google offer unprecedented 
insights into user behaviour, significantly expanding the surveillance capabilities of platforms into a behavioural means of production that Shoshana Zuboff (2019) calls surveillance capitalism. These behavioural designs leverage insights into user behaviour to create the most 'engaging' digital media environment possible. The concern is that a hyper-engaging environment goes beyond informing audiences and users, appealing to individual user's personal subjectivities, biases and world views (Pariser 2011). Facebook's designs, data privacy policy, and model of content dissemination have been criticised for enabling the Cambridge Analytica data scandal (Laterza 2018), amplifying the spread of disinformation in the 2016 Brexit referendum in the UK and the 2016 US Presidential election (Ball 2017), and a 2014 emotion contagion experiment, where Facebook attempted to manipulate the emotions of users in particular countries by adjusting their news feeds (Tufekci 2015). Several former Facebook investors and alumni have publicly denounced the social media platform for their potential harms on democracy and children (McNamee 2019; Solon 2017), with former Facebook executive Chamath Palihapitiya claiming he feels "tremendous guilt" about the harms of social media in "ripping society apart" (qtd. in Vincent 2017). Karppi and Nieborg (2020) claim these public confessions by former Facebook executives are morally driven acts that have several implications for technologists and users. First, the confessions serve to exonerate a degree of personal responsibility with regards to driving the development of the attention economy. Secondly, Karppi and Nieborg suggest they produce dystopian and technologically deterministic narratives that overstate the influence of platforms on users, with the added consequence of positioning disconnection as an unattainable ideal for the everyday user.

Previous technology-based practices of disconnection have aimed to displace users from the attention economy. Scholars have observed that disconnection can be an act of 
protest or economic disruption. Two such examples are the digital artworks Seppukoo.com and Web 2.0 Suicidemachine, which were designed to enable Facebook users to commit a 'digital suicide' (Karppi 2018, 2011). Drawing on Galloway and Thacker's (2007, 135-137) considerations of the "tactics of nonexistence", Karppi suggests these artworks were an act of obfuscation, which Brunton and Nissenbaum (2011) define as "the production of false, misleading, or ambiguous data to make data gathering less reliable." The purpose of the digital suicide was to render the user's profile as non-existent, in effect what Ulises Ali Mejias (2013) calls a 'paranode', so that all that remains is "meaningless to the machinic subjectivation of capitalism" (Karppi 2011). Other similar "unmapping[s] of the network" (Mejias 2013) are advertisement-blockers or Dan Schultz's (2017) Internet Noise, a protocol program which sends random searches through a browser window, flooding trackers with irrelevant information and obscuring surveillance practices. By interfering with data collection activities that determine the personalisation and placement of advertisements, the disconnection in these instances is not so much between human and machine but, rather, between the user and the attention economy.

Disconnecting from the attention economy has also been observed as offering an alternative lifestyle for users. Taking inspiration from the 1988 science-fiction cult film They Live, is technology artist and activist Ivan Cash. Cash created the IRL (in real life) glasses, which are stylish sunglasses fitted with polarised lenses that block any transmitted image from LED and LCD screens from being visible (Pardes 2018). Fashion sensitive products like the $I R L$ glasses suggest resistance to the attention economy can be about more than just undermining hegemonic media practices such as data collection. Disconnecting in such a visible way creates the opportunity for a cultural and class statement. In a study on New Yorker hipsters refusing smartphones in favour of feature phones, Portwood-Stacer (2012C, 
1042) contends that elements of the hipsters media disavowal is performative, demonstrating a certain aesthetic taste with regards to media that "signifies something socially or politically meaningful about the non-user." 'Slow media' is another trend that mixes resistance, aesthetic sophistication and media consumption. Slow media is based upon the slow food movement and positions consumerist practices as key to slowing mass industry production or forcing higher ethical standards (Rauch 2018). An example of a slow media product is Delayed Gratification, a print magazine that claims they do not publish a story until six months after the story has broken. In each edition of Delayed Gratification, editors tell their subscribers that their purchase is undermining the 24 hours news cycle (Orchard and Webb 2018), another dominant media logic that has been criticised for reinforcing speed over quality journalism (Lewis, Rao, and Reiley 2015).

\subsubsection{When resistance becomes control}

Tine Syvertsen (2017) argues that media resistance has historically provided insights into our understanding and use of media. To her point, smartphone resistance in the 2010s is often framed as a self-focused activity to encourage restrained use. Moreover, emphasis is placed on what the individual can gain from disconnecting from the attention economy, rather than disconnection as a collective or societal issue. In a historical study of the swear jar, Ethan Plaut (2015) observes how such a technology acts as a commitment device to incentivise the user from acting beyond their better judgement. Plaut connects the swear jar to the wider issues of 'digital noise pollution' and ubiquitous connectivity, arguing that individual technologies play into the neoliberal, or market-driven project of privatising solutions to what could be conceived as a social problem. The burden of resisting the attention economy is placed on the individual by promising empowerment through limitation. 
In these instances, disconnecting to take back control becomes less about resisting the societal effects of the attention economy, and more about resisting an individual's own urges.

Scholars also flip the notion of disconnection around, asking what technology-based disconnection reinforces or brings users closer to. For example, digital labour scholar Melissa Gregg (2018) argues digital disconnection reinforces the ethos of productivity. Gregg calls productivity apps that restrict access to the internet the latest example of 'efficiency engineering', a term she uses to relate disconnection to managerial practices that employers have been driving on the factory floor, such as Taylorism and Fordism. Disconnection as a variety of efficiency engineering targets knowledge workers-professionals such as lawyers or accountants who are expected to use their cognition for their job-instructing them to concentrate and block out distractions. Kuntsman and Miyake $(2019,910)$ similarly observe that:

The apps position themselves as aids in reducing the digitality of the everyday; however, in fact, these are tools of disciplining a productively labouring subject, one that manages their time effectively, works without distraction and rests well in order to return to an even higher productivity-all with the help of the latest digital solution.

Disconnecting students or workers may benefit from blocking out distractions by seeing improvements to their productivity. Yet educators and employers get to have their cake and eat it too-a disciplined subject who on one level knows how to tap into their networks for information, and on another, to switch off when needing to focus (Beattie 2018b). Digital disconnecting in this instance becomes about enhancing productivity and resisting distractions and the impulsive brain (Guyard and Kaun 2018). The disciplinary potential of disconnection could explain why conservative media advocacy group Common Sense Media and Missouri Republican Senator Josh Hawley view distraction and regulating the attention 
economy as a critical issue. In 2019, Hawley unveiled the Social Media Addiction Reduction Technology Bill (2019), which aims to ban design features such as the bottomless newsfeed and video autoplay. A generous interpretation of the broad interest in regulating the attention economy is that the effects of the attention economy are so widely felt that the issue transcends bipartisan politics. Or, as Gregg (2018) points out, disconnection is often less about what we actually disconnect from and more about what the disconnection functions for. In other words, rather than undermining power, disconnection as self-control reinforces regimes of productivity. In an opinion editorial on his proposed bill, Hawley (2019) demonstrates his disdain for social media by comparing it to a "parasite on productive investment, on meaningful relationships, on a healthy society." Reflecting on the activities that Facebook deploy to prevent end user disconnection, Karppi $(2018,6)$ argues that disconnection is "an issue of power, design and governance over users." Perhaps the same could be said for technological practices that enable disconnection.

Existing research on disconnection and the attention economy offers critical insights about the manufacture of disconnection. According to researchers and practitioners, the attention economy structures the internet in a way that targets and diminishes human agency and democracy. Previous technologies of disconnection have displaced users from the attention economy, with some focusing on enhancing the lifestyles of users, promising users greater self-control and the opportunity to enhance their productivity. Scholars have subsequently enquired into who is driving the disconnection and what regime of power is actually being disconnected from or being reified. These issues of control, power and governance are even more apparent when outlining what the manufacture of disconnection offers to media studies. 


\subsection{Pioneering a digital culture}

The Silicon Valley disconnectionists are experimenting with technology and the practice of disconnection. I consider these technologists to be a 'pioneer community' (Hepp 2016) of digital technology and culture, as they are pushing ideas and norms in relation to digital media technologies in unique directions and further extending the technological into the realm of disconnective practice. Yet the reasons why or how these disconnectionists are resisting the culture of connectivity have yet to be sufficiently answered. More than that, their inventions are novel mixes of technical protocols and behavioural science that have several implications for media studies. Firstly, the manufacture of disconnection challenges established notions of what media are made for. According to Mack Hagood (2019) digital media studies has an infocentric tradition that views media technologies as disseminators of information to convey interpretable messages to audiences. Infocentrism is perhaps due to an Anglo-American tradition in media studies that emphasises discourse or content analysis in analysing media technologies (Siegert 2015). Technologies designed to encourage disconnecting from the internet are for the most part non-infocentric media that are "beyond messages" and more broadly constitute "our infrastructures of being, the habitats and materials through which we act and are" (Peters 2015, 14-15). Similar to ambient media (McCullough 2015) or noise-cancellation or suppression media (Hagood 2019), the point of technologies of disconnection is often not to convey interpretable messages to audiences, but to more meticulously organise the digital environment. In other words, what is at stake when disconnection is manufactured is the potential of technology to better shape the built environment and any human and nonhuman activity within it. 
Secondly, the manufacture of disconnection questions scholarly assumptions regarding connectivity and the use of digital media. Digital or social media studies predominantly emphasise connectivity (Light 2014) and risk what network theorist Mejias (2013) calls nodocentric thinking, whereby scholars only see other nodes or elements of connectivity, which renders disconnective acts as invisible. A study that spotlights disconnection renders certain disconnective acts as visible or observable, offering a critical component to media studies. The manufacture of disconnection challenges the nodocentric assumption that people and services are, and want to be networked by default (Fotopoulou 2014) and identifies the nuances and inequities of disconnection that occur in everyday lives. Put slightly differently, how media technologies intermediate and change social and communicative processes has been a key focus since the inception of media studies (Couldry and Hepp 2017). It remains unclear how software and digital technologies shape and influence disconnective practices. Emphasising the disconnections that people undertake with and/or through media technologies may help to change the historical perception that media studies is uninterested in disconnection and therefore incapable of unearthing insights about associated practices of media resistance (Syvertsen 2017).

Moreover, this type of non-infocentric and connection-limiting media is increasingly common and in demand. Well-known artists such as comedian Hannah Gadsby and performer Childish Gambino (Donald Glover) insist on phone-free performances because they find smartphones in the audience detract from their performance (Smith and Keane 2019). In Australia, the states of Victoria and New South Wales are contemplating phone bans in classrooms to enhance in-person teaching (Merlino 2019). In a study into smartphone use at sports stadiums, Hutchins (2016) concludes that an environment affording unfettered connection (e.g., free Wi-Fi at a sports stadium to encourage social media interaction) 
undermines shared social experiences (i.e. watching the live event). Similarly, Light and Cassidy (2014) argue that social media platforms could be interested in offering affordances of disconnection to create a more balanced communicative experience, increasing user trust and preventing users from undertaking a permanent and irreversible disconnection (such as account deletion). While business and government interest in disconnection could risk diluting disconnection as a practice to resist or disrupt certain regimes of power (Hesselberth 2017) such interest is evidence of the broad appeal of disconnective practice across the whole spectrum of communication.

Studying disconnection therefore offers potential insights into alternative, and arguably less exploitative, ways of living with digital technologies. As Morozov (2014) and Silverman (2015) contend, some businesses significantly benefit from ubiquitous connectivity, and further questions should be asked whether technology platforms such as Facebook are working to ensure that constant connectivity is a central and necessary component of political practice (see Rider and Murakami Wood 2018), aspirational technology use (Docherty 2020) and digital culture more broadly. If digital culture is defined "as an emerging set of values, practices, and expectations regarding the way people...act and interact within the contemporary network society" (Deuze 2006, 63), then the manufacture of disconnection serves to expand scholarly understandings of digital culture by identifying any values, practices, or beliefs in relation to how people disengage within digital and networked media environments. Studying disconnection may offer a more accurate understanding of how people negotiate with digital technologies in their day-to-day lives. At a 2010 technology awards ceremony, Facebook CEO Mark Zuckerberg famously stated that privacy was no longer a "social norm" (Johnson 2010). Similar to groups like the Anonymous hackers, the Silicon Valley disconnectionists are technologists whose inventions and practices offer the 
opportunity to understand how dominant communicative norms and practices are being contested and new intellectual sites of ideological resistance are opening up. At the very least, manufactured disconnection is an attempt to push back against identified $21^{\text {st }}$ century issues such as information overload, privacy erosion, ubiquitous surveillance, and work and leisure slippages, as well as tangibly re-align the design and goals of technology with the social needs of users.

By analysing technologies and practices that challenge assumptions and conventions about connectivity and information, I look to carry on the media studies tradition of interdisciplinarity. Media studies has always, in all its traditions and locations, looked outside existing media theory and its own field to understand new phenomena (Schirato et al. 2010). I require an approach that does not view digital technologies through an infocentric or a nodocentric lens. I therefore look towards theories concerning power, technology and selftransformation to analyse the manufacture of disconnection. In the next chapter, I outline my research questions, theoretical framework and methods to study the manufacture of disconnection and the shaping of our mediated environment and selves. 


\section{Research design and method}

In this chapter, I outline my approach to investigating the manufacture of disconnection. My research design contains three components: the research questions that I ask, the theoretical framework that informs my analysis of the Silicon Valley disconnectionists and their technologies of disconnection, and the methods that I employ to answer the questions. I open the chapter by outlining my research questions and briefly discuss how they build upon the paradox of dis/connection, a major issue within disconnection scholarship that I discussed in the first chapter. I then turn to my theoretical framework which draws upon the field of French philosopher and sociologist Michel Foucault (1926-1984). I believe that his scholarship offers a unique theoretical framework for the manufacture of disconnection. Unlike philosophers such as Henry David Thoreau ([1854] 1995) or, more recently, Robert Hassan (2019)', Foucault never overtly practiced or experimented with disconnecting from society, technology or other people. Neither did Foucault pine for a qualitatively different technological society like Martin Heidegger ([1927] 1953) or Jacques Ellul (1965). But Foucault did contemplate, and was affected by, technology. In particular, Foucault's dichotomous relationship with technology and the influence technology had on his conceptualisations of powerand resistance make him an ideal theorist to interrogate the manufacture of disconnection. I discuss Foucault's relationship with technology, and how this view connects Foucauldian power, subjectivity and resistance in a way that is relevant to the Silicon Valley disconnectionists and their inventions. Throughout my discussion of late Foucault, I outline the importance of

\footnotetext{
1 Thoreau is famous for retreating to a cabin in the woods in Concord, Massachusetts in the early $19^{\text {th }}$ century. Hassan is a media philosopher, who in 2019, undertook a six-week voyage on a commercial freighter ship from Melbourne to Singapore without any networked technology.
} 
subsequent scholars such as Deleuze, Nikolas Rose and Lois McNay, who furthered and critiqued Foucault's ideas on power and resistance in the context of new technological developments and different contexts. Following this, I outline why semi-structured interviews with the Silicon Valley disconnectionists and applying the walkthrough method (supplemented by a platform studies approach) to their inventions are suitable for unravelling the manufacture of disconnection.

\subsection{Research questions: reframing the paradox of dis/connection}

My research questions are informed by a major issue about disconnecting from the internet that I discussed in the first chapter: the paradox of dis/connection. In the previous chapter, I summarised Hesselberth's (2017) paradox of dis/connection as a critique of disconnection scholarship and suggested that it has implications for a study on the manufacture of disconnection. The implication is that the manufacture of disconnection insufficiently divorces the concept of disconnection from connection and that any technologised disconnection reinforces, rather than resists the attention economy. While I criticised the paradox for unnecessarily framing disconnection in binary terms and black boxing the technological side of disconnecting, I acknowledged that the paradox is a useful reminder to assess what is actually being disconnected from. My research design is therefore informed but not deterred by the paradox of dis/connection. In this section I reframe the paradox of dis/connection into a set of research questions to critically assess the manufacture of disconnection as a means to resist the attention economy.

My first research question asks: What types of relations do technologies that manufacture disconnection produce? The manufacture of disconnection may be paradoxical by requiring users to further connect to disconnect, but I believe this paradox is instructive, 
rather than antithetical, to my thesis. There are ample technological paradoxes in media technologies: for example, the internet as a tool of both freedom/control and democracy/surveillance (Chun 2006, 2016; Morozov 2011). Wendy Hui Kyong Chun (2016, 19) views technological paradoxes as central to the allure and generative power of new media, and that breaking out of any paradox requires "inhabiting networked vulnerabilities" and a "reimagination of networks." What Chun suggests is not to avoid a technological paradox, but to explore the new modes of relations that the paradox can generate. In other words, paradoxes are not impasses, but create opportunities for further analysis. Taina Bucher $(2020,615)$ makes a similar point, arguing that if digital disconnection can be both an act of resistance and type of media use, then scholars need to identify the kind of relations that digital disconnection produces: "The question is how to conceptualise digital disconnection when the very acts of avoidance and disengagement may place the actor not on the outside of algorithms but on the very inside." Perhaps a means to find a limit to connectivity culture is not outside of connectivity, but inside the relations that the manufacture of disconnection generates.

The paradoxical nature of disconnection as both an act of resistance and media use leads to my second research question, which is: How do these relations implicate resistance to the attention economy and culture of connectivity? If resistance to the attention economy can be enacted from an inside position by technologists who operate under the logics of the attention economy, then greater attention should be paid to what aspects of the attention economy are actually being resisted and conversely, which parts of the attention economy are being extended. In a study comparing Anonymous hackers to 19th century Luddites, Deseriis (2013) demonstrates that different relations with technology can lead to alternative ways of resisting technology. Despite both groups sharing similar goals in sabotaging 
machines, Deseriis (2013) argues that Luddite subjectivity resists technology by being in strict opposition to technology, while the Anonymous hacker subjectivity is constituted via a mastery over information technology. If we similarly make visible the architectures of the relations produced by the manufacture of disconnection and assess how these relations with technology offer means to disconnect, we can identify types of disconnective practice that cannot be controlled by, or are not desirable to, a platform. Doing so will allow us to continue to "trespass into the culture of connectivity and do violence to the dominant image of thought" (Karppi 2018, 3). While the dominance of connectivity in our culture is well established (van Dijck 2013) perhaps we should likewise 'do violence' to the idea that all types of manufactured disconnection are inauthentic. In doing so, we may be able to understand with greater precision as to what aspects of the attention economy and the culture of connectivity are being undermined, and conversely, how these regimes of power are being extended. As Judith Butler $(1997,100)$ puts it, we can "work the power relations by which we are worked". Following these observations, I interpret the paradox of dis/connection as an invitation to identify and critically assess the new modes of relations that the manufacture of disconnection generates. Answering these research questions, however, first requires a suitable theoretical framework that addresses power relations, technology and resistance.

\subsection{Theory: late Foucault on power, technology and resistance}

Foucault provides a useful framework for a study on the manufacture of disconnection because of his unique perspective on how to resist the forces he believed were shaping society and individuals. Throughout his entire scholarship, Foucault sought to identify how people were shaped by power. Following his doctoral thesis on the history of mental illness, Foucault's early scholarship focused on the implications of Western thought 
systems on institutions and people. In the 1960s, Foucault traced the Western development of 'madness' in Madness and Civilisation ([1960] 1988) and, more broadly, systems of knowledge and norms in The Order of Things ([1966] 1994) and The Archaeology of Knowledge([1969] 1982). In the 1970s, Foucault analysed the development of modern power and the forces, structures and techniques that determine the modern human subject in Discipline and Punish ([1975] 1977) and The History of Sexuality: Volume /([1976] 1990). It was not until The History of Sexuality: Volume //([1984] 1990) and Volume ///([1984] 1988) that Foucault directly addressed the way human beings can resist structures of power. The last period of Foucault's scholarship where Foucault turned to explicitly conceive of modes of resistance to power is referred to as 'late Foucault' (Elden 2016; Golder 2015; Zamora 2019b). That Foucault did not spend as much time directly on resistance in comparison to his early work on knowledge and power is not a weakness of his perspective on resistance. Foucault extensively wrote about power, knowledge and the genealogy of the modern human subject. It is precisely because of Foucault's thorough understanding of the ways that people can be turned into subjects that makes his perspective on resistance so unique and valuable.

The influence of Foucault's later work only grew after his death in 1984. Several posthumous publications-most notably Foucault's series of lectures at the Collège de France in the 1970s and 1980s and The History of Sexuality: Volume IV(2018)-further revealed connections between Foucauldian power and resistance. Late Foucauldian scholars take a more global view of Foucault's scholarship and consider his turn to resistance in the 1980s to be an opportunity to reinterpret his earlier work on the shaping of the modern subject. Such a view stands in contrast to more orthodox uses of Foucault, which discuss his concepts in isolation from each other. In the following sections, I outline why a late Foucauldian approach is useful to theorise the manufacture of disconnection, starting with 
an overview of Foucault's conceptualisation of power and the influence of technology in shaping society and individuals.

\subsubsection{Technologies of power}

Foucault's views towards power were influenced by technology; however, his use of the term 'technology' was ambiguous. When Foucault wrote about technology, he was often referring not only to engineered things or material objects, but also practices in the medical or psychological sciences and self-care. Foucault's interpretation of technology is, in part, due to the French word technologie being closer to 'technique' than the English version of technology (Behrent 2013). Although Foucault did not mention technology in his scholarship until The History of Sexuality: Volume // ([1984] 1990), Michael Behrent (2013) argues that new technologies in post-World War II France influenced his early thinking. Foucault's formative intellectual years occurred during a time in France when new types of technologies such as Taylorism and other industrial psychology systems were implemented across France (Behrent 2013). Foucault was trained in experimental psychology and, like other prominent French intellectuals during the 1950s such as Michel Crozier or Jacques Ellul, was extremely sceptical towards technocrats and techniques of management that claimed to be able to scientifically organise workers and the general population (Behrent 2013). What separates Foucault from other French intellectuals, however, was that Foucault did not take issue with technology per se, but with how technologies enabled power to operate.

Foucault believed that technology orientated power. Peter-Paul Verbeek (2011) compares Foucault's view on technology to the hermeneutic, or interpretative, approach of Martin Heidegger (1977). According to Verbeek, Heidegger articulated a technological way of interpreting reality, seeing technology as pervading social structures and knowledge systems. 
In contrast, Foucault formulated an understanding of reality as structured by power-a radical view that considers power omnipresent, congealed to and manifesting as knowledge, technologies and practices. Foucault's view on power is seminal to media studies and the social sciences because he departed from the idea that power was a coercive force wielded by the sovereign state, seeing power instead as distributed and pervasive and manifesting in knowledge, discourses and practices. ${ }^{2}$ Throughout his scholarship, Foucault analysed structures of power in knowledge, practices and ideas. In the 1960s, Foucault ([1960] 1988) argued that knowledge and power were conjoined, with the former as an action or manifestation of the latter. In later decades, Foucault would identify technology as another realisation and development of power in modern society, which he described as 'technologies of power' (Foucault 1988). In his view, then, technologies provided an orientation to power, disciplining, organising or normalising human subjects. Foucault's articulation of the concept of technologies of power helped him to emancipate his concept of power from juridical representation. Rather than limiting his perspective on power to that which operates in laws, codes and statues, the concept of technology enabled Foucault to observe how power shapes the human subject beyond the law (Foucault 2005). Technology is therefore a critical concept for Foucault that unifies his scholarship with regards to the shaping of the human subject, a view that is present in his well-known discussion of Jeremy Bentham's panopticon prison (Foucault [1975] 1977).

The panopticon prison is worth outlining in detail as it has particular relevance to the Silicon Valley disconnectionists. The panopticon was a disciplinary concept created by

\footnotetext{
2 Some scholars argue that Foucault saw power too broadly or as a monolith. For example, power theorist Steven Lukes (2005) contends Foucault's omnipresent view of power is so encompassing that it loses analytic purchase. Fredric Jameson (1984) similarly points out that resistance becomes increasingly difficult the more omnipresent power becomes.
} 
Bentham where a central observation tower was placed in the middle of a ring of prison cells; from within the tower, a single guard could see every cell and inmate but the inmates cannot see inside the tower. In Discipline and Punish([1975] 1977), Foucault describes the panopticon as having the effect of inscribing a gaze onto prisoners which would in turn encourage them to govern themselves. The internalisation of governance by prisoners shaped their identity as observable and knowable subjects, which Foucault argued would have an effect on their behaviour inside the prison cells. Foucault's conception of a technology of power is similar to the Silicon Valley disconnectionists' concerns about hijacking the minds of people who use their technological inventions. Analogous to Foucault's interpretation of the panopticon, users of social media platforms like Facebook inhabit an architecture and digital space where they are subjected to the power to judge themselves and others. As a social media platform, Facebook is a 'playground' type of place where scores of people hang out and identity formation occurs (Aiken 2016; Turkle 2013). In this visible place, the behaviour of some inhabitants can resemble those of actors who tailor their performance to the audience. Visibility has a normative effect on behaviour in the sense that inhabitants feel compelled to participate online or otherwise risk becoming socially invisible (Bucher 2012), and adjust what they like and the substance of their comments, knowing their behaviours have the potential to be observed by others. In other words, in visible places such as Facebook, users may feel pressured to use the platform, and pander to the crowd and inhabit a performative subject position, crowding out other, perhaps more 'interior' aspects of their identity (Alter 2017; Turkle 2013; Twenge 2017). Of course, the performative self predates Facebook and other social media platforms; nonetheless, the panoptic architecture of social media encourages user performativity by turning it into a subject position that is part of the platform. 
Importantly, Foucault did not believe that technologies of power always negatively affected people. As Hoy $(2004,6)$ puts it, "for Foucault, all domination is power, but not all power is domination". Foucault's view towards technology and power was more complex. For one, Foucault contended modern technologies of power were different to the techniques of torture and execution under sovereign power that existed centuries before. Modern technologies of power did not conquer the body but rendered it more useful and docile (Foucault [1975] 1977). For example, Foucault observed the potential of technologies of power to transform an individual's understanding of themselves and to produce individuals as objects of knowledge. Similarly, the attention economy does not simply 'conquer' users. Navigating the attention economy can be a pleasurable and informative activity for many. Scrolling social media, reading news headlines and reacting to banner advertisements elicits a range of affective user responses such as excitement, boredom, fear or outrage (Paasonen 2018). The attention economy does not necessarily negatively affect users in a disciplinary way, but rather produces them as emotive or feeling subjects. Put another way, power subjectifies, turning individuals into subjects. Foucault $(1980,119)$ described modern power as the following:

\footnotetext{
What makes power hold good, what makes it accepted, is simply the fact that it doesn't only weigh on us as a force that says no, but that it traverses and produces things, it induces pleasure, forms knowledge, produces discourse. It needs to be considered as a productive network which runs through the whole social body, much more than as a negative instance whose function is repression.
}

However, Foucault's technologies of power are insufficient on their own to capture the effects of the attention economy. Foucault conceptualised his understanding of power in response to the sovereignty of the State and development of modern civil institutions. We cannot expect 
him to fully explain our contemporary predicament with respect to the attention economy. Fellow French philosopher Gillies Deleuze is helpful here. Deleuze (1992) extends Foucault's technologies of power in response to the rise of consumer society, contending that subjects no longer need to be disciplined to be controlled. Deleuze argues that modern power is driven as much by consumer society as it is by civil society, pointing out that the subject of the consumer society is not the individual but the 'dividual', a category of pre-individual, to capture the data that are produced by individuals. Deleuze's dividual is a useful way to understand how the use of data becomes part of the subject making process and informs what kinds of subject positions are available to users (Deseriis 2012). In addition, Deleuze's response to Foucault is helpful considering that the attention economy is a key pillar of consumer society by matching users to potentially relevant consumer products and services (Wu 2016). The knowledge and data that users produce in the attention economy-what users click or comment on, time spent on webpages, and so on-is valuable to advertisers, data analytics businesses, and other third parties, in effect paying for users' access to the attention economy (Crogan and Kinsley 2012). Deleuze demonstrates that the multifaceted nature of Foucauldian power-to simultaneously discipline and produce users as subjects-acquires economic value in the attention economy.

As discussed in the first chapter, the Silicon Valley disconnectionists are concerned by the fact that that the attention economy is designed to optimise economic activity and has created addictive products and services (Courtwright 2019). A Deleuzian perspective on the attention economy suggests that design functionalities such as the bottomless newsfeed and red coloured notifications act as mechanisms of control to ensure user attention is constantly circulated. The attention economy not only produces users as subjects, but also 
'controls' users by facilitating affective circuits of interactivity to promote degrees of lifestyle competition.

Other scholars have expanded Foucault's concept of power and enable a stronger connection between Foucault and the attention economy. One such expansion is the concept of 'psychopower' from Byung-Chul Han (2017), who argues that technologies of power no longer discipline subjects, but subject users to a new discursive regime that he calls the 'achievement society'. A second is 'noopower' from Mauricio Lazzarato (2006), which acknowledges the shift of power from the materiality of the body to the psychic capacities of perception and attention. Robert Gehl (2013) connects these post-Foucauldian descriptions of power directly back to Foucault by arguing that social media has institutionalised the management of end user attention. Users in the attention economy are subjected to a different type of institution, and exposed to a surplus of other people's lifestyles, experiences and material possessions, which act as benchmarks to aspirational social and cultural classes (Gehl 2013). Overall, the concept of technologies of power provides an underlying critical framework that capture the concerns of the Silicon Valley disconnectionists. Drawing upon Foucault's technology of power and subsequent iterations by scholars in the field of Foucault, we can acknowledge that the attention economy shapes users to frequently participate and consume online as well as produce themselves as subjects.

\subsubsection{Technologies of self and space}

What makes Foucault even more relevant to the manufacture of disconnection is how he believed power could be resisted. In the 1980s, Foucault shifted to studying how human beings could resist being turned into subjects of power. Foucault did not believe that people could escape power because he believed power is omnipresent throughout society. Instead, 
Foucault believed resistance must come from within existing power relations. According to Foucault ([1976] 1990, 95-96), "where there is power, there is resistance, and yet, or rather consequently, this resistance is never in a position of exteriority in relation to power". The view that resistance is immanent to power bears similarities to the Silicon Valley disconnectionists, who do not resist the attention economy by retreating from the internet, but rather utilise their technological expertise to disconnect from within it. Here, resistance is an act of intervention or a reverse engineering of the process of being shaped by power. Foucauldian resistance contrasts with traditional political conceptualisations of resistance, which typically entail explicit clashes of power relations, groups of people and worldviews. For example, the non-violent civil disobedience movement Black Lives Matter involves a marginalised group of people overtly challenging the hegemonic ideologies and racist institutions that oppress them. For Foucault, "the question [of resistance] was not to "liberate" people from power, but to invent, within the matrix of existing power relations and positioned on the narrow margin of freedom that we do possess, new forms of subjectivity and new capacities for self-transformation" (Zamora 2019b, 9). Foucauldian resistance is therefore a question of reinvention within the existing power relations in society, rather than overtly opposing them.

Foucault considered resistance to be an ethical exercise, but he did not interpret ethics in the Kantian tradition of moral philosophy or normative conceptions of right or wrong. In fact, Foucault (1982) criticised Immanuel Kant's construction of moral philosophy and ethics and contended that any conception of morality, such as Enlightenment humanism, was already shaped by power and therefore inherently problematic. Instead, Foucault refused to acknowledge an a priorior ahistorical conception of the subject. For most of his scholarship, Foucault declared that the freedom so integral to modern philosophy since the 
Enlightenment was a façade. As discussed in the previous section, the assumed 'autonomous subject' is in fact a subject produced by technologies of power characteristic of modern society. As the subject was already determined by power, Foucauldian ethics became an exercise in developing a relation of self to itself in terms of its moral agency (Foucault [1984] 1990).

To demonstrate how individuals could conduct themselves ethically or resist power, Foucault shifted his attention from technologies of power to technologies of the self. A technology of the self is an exercise that reverse-engineers technologies of power to enable individuals to make themselves the object of their own practices. A technology of the self, according to Foucault $(1988,225)$, is a critical relationship with the self that

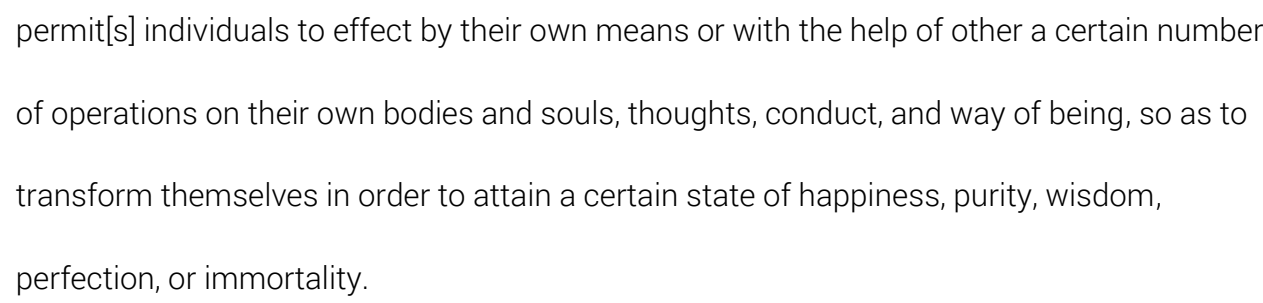

Foucault analysed technologies of the self in ancient Hellenic and Roman contexts. To the ancient Hellenic and Roman philosophers, technology, or techne, was synonymous with aesthetics and caring for the self, a process by which one treats one's life as a work of art. For example, Foucault ([1984] 1988) saw the purpose of the Hellenic practice of askēsis as not eliminating all sexual desires for pleasure but instead training the individual to temper their desires in the service of a wider goal. Another similar technology of the self that Foucault discussed was Hupomnemata or self-writing. Foucault (1997) describes how ancient practices of Hupomnemata -the self-writing of letters, notebooks, treatises, or diariesinvited introspection, new forms of experiencing the self, as well as making sense of a diverse set of ideas or events. Foucault $(1988,27)$ explains how writing about the self was an 
important aspect of taking care of oneself: "taking notes on oneself to be re-read, writing treatises and letters to friends to help them and keeping notebooks in order to reactivate for oneself the truths one needed". In a study on PhD students and blogging throughout their doctorate, Mewburn and Thomson (2017) observed that blogging functioned as a technology of the self by helping students to reflect on their own scholarly habits and experience. Furthermore, blogging aided students to create a scholarly persona and resist dominant academic textual genres and conventions in their writing. The authors also contend that blogging creates opportunities for a student to socialise their ideas and experience outside of the traditional supervisor-student relationship and could combat isolation, a common experience for PhD students (Owler 2010).

Existing disconnection research has applied askēsis to theorise disconnection. Laura Portwood-Stacer (2012b) argues that National Unplugging Days and digital detoxes are evidence of disconnecting from the internet being framed as an ascetic exercise as smartphone users are motivated during the detox to reflect about their technology habits. Disconnection as resistance in the Foucauldian context appears to be about the enlargement of the possibilities for self-determination and asking what new types of subjectivities can be constituted within the existing paradigm of society. Foucault ([1976] 1990, 13) argued new subjectivities or a critical relation with the self could serve "as functional devices that would enable individuals to question their own conduct, to watch over and give shape to it, and to shape themselves as ethical subjects". In particular, Foucault $(2005,94)$ considered that technologies of the self could transform an individual to become more resilient to unexpected hardship or trials, arguing that "[t]he practice of the self is established against a background of errors, bad habits, and an established and deeply ingrained deformation and dependence 
that must be shaken off". In summary, technologies of the self offer a mode of resistance because they enable individuals to shape their existence before external forces get there first.

But not all Foucauldian resistance starts from the self. A lesser-known mode of Foucauldian resistance is opening up spaces of freedom and experimentation. During the 1970s, Foucault protested for the gay liberation movement and advocated for spaces where inhabitants can resist subjectification or regimes of normalisation. Foucault $(1993,252)$ considered that space was instrumental in informing communal, urban and public life, arguing that "space is fundamental in any exercise of power"-his work in this area influencing critical approaches in architecture and urban studies. In addition, Foucault identified disciplines such as criminology, education and psychiatry that provided a 'discursive regularity', or an ordering of things, and contended the process of subjectification was influenced by related 'normalizing' government policies (Beckett, Bagguley, and Campbell 2017). In response, Foucault referred to "creating new cultural forms" and "invent[ing] new gay lifestyles" that disrupted the usual order of things (qtd. in Barbedette 1982, 36). Beckett, Bagguley and Campbell $(2017,172)$ argue that Foucault was seeking to "understand how a tear, or a rupture in this order of things emerges, how resistance towards the normalizing rationales of government can occur." In his articulation of heterotopias, or other spaces, Foucault envisaged different modes of truth, that provide means of escape from the norm. Foucault saw heterotopias as transformative spaces that threaten the dominant ordering of things and allow inhabitants to think and act differently and produce new subjectivities. ${ }^{3}$ Late Foucault scholarship on self-practices enables a reinterpretation of his earlier work on heterotopias as spaces of resistance. While Foucault never referred to a technology of space

\footnotetext{
${ }^{3}$ I discuss Foucault's heterotopias in more detail in chapter three.
} 
as a mode of resistance, we can infer from his discussion of heterotopias and spaces of experimentation an interest in spatial-based resistance.

Late Foucauldian scholars contend that Foucault's perspective on technologies of power and turn to resistance explain his ambivalent and contentious views on neoliberalism. Foucault did not approach neoliberalism as economic logic but as a political ontology, or a framework to conceive how individuals were shaped by power in society. In Foucault's published series of lectures at the Collège de France, Foucault $(2008,260)$ described neoliberalism as a "technology of the environment" that signals a "massive withdrawal with regard to the normative-disciplinary system." Zamora (2019a) further explains why Foucault was not deterred by neoliberalism, writing that "Foucault was never a neoliberal in the strict [economic] sense of the term, his understanding of power and resistance in his last decade resonates profoundly with neoliberal political ontology and its transformation of politics into ethics." The appeal of a neoliberal society to Foucault was a decrease of the jurisdiction of the moral by state institutions whereby individuals were freed from the "type of individualization" associated with the state, rather than the state and state institutions themselves (Zamora 2019a,60). In short, neoliberal societies are an example of Foucauldian resistance in action where individuals are less shaped by normalising power that is adjudicated by the state. Zamora (2019b) suggests we can relate Foucault's concept of disciplinary power that he identifies in Discipline and Punish ([1975] 1977) and his ambivalence towards neoliberalism (2008) as an attempt to imagine a mode for subjects to be less governed.

Foucault's nuanced understanding of power, technology and resistance provides a robust theoretical framework for the manufacture of disconnection. Foucault rejected the idea of an autonomous moral subject or that any individual could ever be totally disconnected 
from power. Instead, Foucault was a relational thinker who interpreted society, knowledge, and even resistance as constituted by power relations. Yet Foucault believed individuals could have a different relationship to power. He identified technologies of self and space that could disconnect individuals from the norms of authorities by enabling the individual to intervene in the subjectification process. Therefore, from a Foucauldian perspective, the terms connect and disconnect acquire new meaning. Connection and disconnection are less oppositional communicative states that indicate degrees of proximity to mobile or Wi-Fi networks or other mediated environments, and more actions to reorganise relationships to power. Following Karppi $(2018,103)$, I interpret disconnections as "breaks, ruptures, and interruptions that shape, transform, and mutate the formation of our current culture" rather than a binary communicative state that is in strict opposition to connectivity. These types of disconnections enable users to have a more critical relationship to connectivity by enabling the user to change the qualitative dimensions of their internet and social connections, rather than severing them entirely.

In addition, drawing upon Foucault's technologies of space and self to theorise the manufacture of disconnection provides an opportunity to expand understandings of how disconnection is undertaken with technology. So far, scholarly understandings of disconnecting with technology is limited to Light's (2014) analysis on disconnective practice, which focuses on user and non-human modes of disengagement within social media, such as blocking another user on Twitter. The Silicon Valley disconnectionists are expanding the possibility of disconnective practice by creating new modes of disengagement within the attention economy. They have not retreated from the internet or their technological practices entirely; neither are they trying to dismantle the attention economy or position users outside of the internet. Instead, these disconnectionists are repositioning users inside the attention 
economy is new ways. In order to avoid the subjectification by the attention economy, the Silicon Valley disconnectionists have created new technological relations to produce users to self-manage their behaviours, interactions, relationships, ambitions, desires and so on. Within a Foucauldian framing, these technological relations can be understood as new architectures, digital spaces and subject positions that enable disconnective practice by offering users means to disengage and resist norms that relate to the attention economy, and/or aspects of themselves or communications from other users. These technological arrangements mean disconnectionists are changing the function of digital space, themselves and their users' relationship to the attention economy. To this end, the manufacture of disconnection matches what Ettlinger (2018) calls 'productive resistance', where digital governance is resisted not by avoiding, disrupting or obfuscating it, but by making use of digital governance in a new way.

Lastly, my theoretical approach is also informed by post-Foucauldian scholars. A significant number of scholars have extended and critiqued Foucault's work, providing an additional means to assess the dimensions of Foucauldian power and resistance. Foucault himself was unclear how resistance was possible against different forms of power (Lilja and Vinthagen 2014), perhaps because he did not wish for his strategies of resistance to become hegemonic or co-opted by power. However, a significant number of post-Foucauldian scholars took up the exercise of developing Foucauldian resistance. I therefore turn to scholars such as, but not limited to, Rose and McNay, who assessed the viability of Foucauldian resistance in their own critiques and expansions of Foucauldian power and subjectivity. These scholars situated Foucault in fields such as feminist studies and the health and behavioural sciences, and extended Foucauldian scholarship by discussing the influence of scientific practice on subjectivity and technologies of power and self. They also 
consider the limitations of Foucault's interpretation of the subject and, therefore, resistance. Furthermore, in some instances in this thesis, I connect Foucauldian space and subjectivity to non-Foucauldian concepts to further Foucauldian resistance in the context of new digital media technologies. As a whole, I use wider scholars' insights on the nuances in which Foucault articulated the operations and regimes of power to contribute to an assessment of the transformative potential of technologised disconnection: what regimes of power are being disconnected from, how so and, in return, what norms or regimes are being connected to or reinforced.

\subsection{Method: identifying and analysing technologies of disconnection}

While I draw upon Foucault to theorise the manufacture of disconnection, I do not use a Foucauldian method to answer my questions. I am not tracing the genealogy of the disconnected subject nor attempting to explain a 'history of the present' in relation to disconnecting from the internet, for example. I am more interested in the power relations embedded in present technologies of disconnection and the technological relations that are being currently constructed by the Silicon Valley disconnectionists. I wish to understand how the disconnectionists are expanding the possibilities to disconnect with technology and know what is actually being disconnected from. To critically assess the inventions of the Silicon Valley disconnectionists and understand them as modes of resistance, I undertook three major tasks: talking to the Silicon Valley disconnectionists to provide wider context to the manufacture of disconnection and choose specific case studies, analysing selected technologies of disconnection, and reflecting about my own positionality in relation to disconnection. 


\subsection{Semi-structured interviews}

The first stage of my research process involved undertaking in-depth and in-person interviews with the Silicon Valley disconnectionists. In the first chapter, I described Silicon Valley disconnectionists as a loosely affiliated group of technologists based in the United States that share concerns about the effects of the attention economy and constant connectivity to the internet, and are inventing technology-based ways to disconnect in response. An in-person interview approach enabled me to gain a perspective beyond the current frameworks in which disconnection is normatively conceptualised (e.g. Hesselberth 2017), and shift to emphasising how the Silicon Valley disconnectionists perceive disconnecting from the internet (Qu and Dumay 2011). I approached these interviews using Brinkmann and Kvale's (2014) seven stage recommendations for undertaking interviews: thematisation, design, interview, transcription, analysis, verification, and reporting. The thematisation of the interview included identifying the purpose of the interview, the themes to be investigated, possible hypotheses, as well as choosing the disconnectionists to be interviewed. I undertook desktop research to identify potential disconnectionists who were creating technologies of disconnection in the United States. I selected participants using the following criteria: anyone who had invented a technology of disconnection, was a senior member of an organisation that had made a technology of disconnection, or was a public advocate for using technologies of disconnection to disconnect from the internet. While technologies of disconnection have been created in places such as Europe, Asia and Australia, I chose to limit the disconnectionists to those based in the United States which allowed me to focus the manufacture of disconnection within the context of Silicon Valley technology culture. That being said, Silicon Valley disconnectionists are not specifically tied to the physical location of Silicon Valley and live in other parts of California or the United 
States more broadly. I discovered that the manufacture of disconnection was taking place beyond Palo Alto or the San Francisco Bay Area and that the Silicon Valley is a catchall term for a diverse area of technological innovation that embodies the ethos of Silicon Valley culture (Fisher 2018; Levina and Hasinoff 2016). Nonetheless, choosing to focus on Silicon Valley disconnectionists and their technologies of disconnection meant that my analysis of the manufacture of disconnection is limited to the United States.

I identified a pool of fifteen disconnectionists in total using the above criteria. I contacted all potential people by email and interviews were confirmed via a Microsoft Outlook calendar invite. I interviewed nine participants, which is considered an appropriate number for qualitative research to obtain substantial interview material (Brinkmann and Kvale 2014). Interviews were conducted in September 2018 in Los Angeles, San Francisco and San Diego in California, and Denver, Colorado. One interview was undertaken over Skype with the disconnectionist in New York at the time. The nine final participants are listed below in Table 2-A. 


\begin{tabular}{|c|c|c|c|}
\hline Person & Location & Technology of disconnection & Detail \\
\hline $\begin{array}{c}\text { Max } \\
\text { Stossel }\end{array}$ & $\begin{array}{c}\text { San } \\
\text { Francisco }\end{array}$ & $\mathrm{n} / \mathrm{a}$ & $\begin{array}{l}\text { Chief spokesperson for The Center for Humane Technology, a Silicon Valley non-profit organisation that } \\
\text { seeks to reform the attention economy by demanding a 'Hippocratic Oath' between designers and users, } \\
\text { new design standards as well as a Bill of Rights for attention. }\end{array}$ \\
\hline $\begin{array}{l}\text { Charlie } \\
\text { Stigler }\end{array}$ & $\begin{array}{c}\text { San } \\
\text { Francisco }\end{array}$ & Self-Control & $\begin{array}{c}\text { Programmer behind Self-Control, a program that blocks access to specific websites for a allocated period } \\
\text { of time. }\end{array}$ \\
\hline $\begin{array}{l}\text { Geza } \\
\text { Kovacs }\end{array}$ & $\begin{array}{l}\text { Stanford } \\
\text { University }\end{array}$ & Habit Labs & $\begin{array}{l}\text { PhD candidate at Stanford University and software developer behind Habit Labs, a web browser extension } \\
\text { that helps users to disconnect from specified websites. }\end{array}$ \\
\hline Joe Hollier & $\begin{array}{l}\text { New York } \\
\text { (via Skype) }\end{array}$ & Lightphone & Founder and CEO of the Light Phone, a luxury feature phone that can only transmit SMS and calls. \\
\hline $\begin{array}{l}\text { Andrew } \\
\text { Dunn }\end{array}$ & $\begin{array}{c}\text { San } \\
\text { Francisco }\end{array}$ & Siempo & CEO of Siempo, a distraction-free based interface for Android smartphones. \\
\hline Chris Bolin & Denver & The Disconnect & $\begin{array}{l}\text { Digital artist and programmer and the founding editor of The Disconnect, a digital magazine that forces } \\
\text { readers to disable their internet connection. }\end{array}$ \\
\hline $\begin{array}{l}\text { Ramsay } \\
\text { Brown }\end{array}$ & $\begin{array}{c}\text { Los } \\
\text { Angeles }\end{array}$ & Space & $\begin{array}{c}\text { CEO and founder of Boundless Mind who developed Space, an app that breaks technology-based habits } \\
\text { smartphone. }\end{array}$ \\
\hline $\begin{array}{l}\text { Kim } \\
\text { Cavallo }\end{array}$ & $\begin{array}{l}\text { Los } \\
\text { Angeles }\end{array}$ & Lilspace & CEO and founder of Lilspace, an app to help users spend more time off their smartphone. \\
\hline $\begin{array}{l}\text { Zack } \\
\text { Prager }\end{array}$ & San Diego & Ransomly & $\begin{array}{c}\text { CEO and founder of Ransomly, which makes hardware devices that smother the connective capability of } \\
\text { any smart device in its range. }\end{array}$ \\
\hline
\end{tabular}

TABLE 2-A: INTERVIEWED DISCONNECTIONISTS AND THEIR TECHNOLOGIES OF DISCONNECTION

${ }^{4}$ Max Stossel is a publicly recognised disconnectionist who has delivered popular TED talks and been featured in the mainstream press. He is what Kvale and Brinkmann (2009) would consider an 'elite interviewee', and I therefore prepared to combat any prepared-in-advance 'talk tracks'. 
Interviews were designed to acquire specific knowledge about the historical, cultural and industry context of manufacturing disconnection. A list of the topics I discussed in the interviews is available in Appendix One. Given technologies of disconnection are emergent and diverse, the interviews were semi-structured and canvassed a range of topics, including: problematising and resisting the attention economy; reflections about Silicon Valley culture; philosophies or motivations that inspire the manufacture of disconnection; mission statements or governance models; personal disconnective practices and experiences; if they use their technologies of disconnection, and if so, the contexts and how they use them. The interviews opened with a briefing, confidentiality statement, and a request to record the proceedings with a Dictaphone. The interviews ranged from 90 minutes to 150 minutes in length and were conversational to allow learned knowledge to steer the interview and inform the research. The interviews closed with a debriefing where the participant was informed of the next steps and invited to share their experience and offer any closing comments.

The interviews were transcribed and coded using NVivo software. I chose thematic analysis to capture patterns across my qualitative dataset because of the flexibility of the method and compatibility with a wide range of theoretical frameworks (Braun et al. 2019). I identified 1086 unique codes across my interview data, and from this, generated some highlevel themes about the Silicon Valley disconnectionists and their technologies of disconnection. These broad themes included the technical components that enable digital disconnection, the importance of user subjectivities, what disconnecting meant to the disconnectionists or how they preferred to frame disconnecting from the internet, the aspirations of their technology of disconnection or business, and the issues with Silicon Valley technology culture and the attention economy. 
I then selected five technologies of disconnection as case studies for further analysis. My process of selection was initially practical: of the nine interviews in total, only seven had technologies of disconnection that were available to analyse. ${ }^{5}$ The process to narrow down the remaining seven technologies to case studies was determined by my late Foucauldian theoretical framework of spatial and self-based resistance. I chose two technologies where the utilisation of space appeared to be integral in the construction of disconnection, and three technologies of disconnection that focused on disconnecting the user from an aspect of themselves or the internet, rather than from the internet as a whole. To assist this selection process, I drew from my interview data with the disconnectionists and wider industry and popular discourse to provide more detail about the technology of disconnection in question. This allowed me to consider issues such as why the disconnectionist had created the technology of disconnection, the relevancy of space and/or self in the construction of disconnection, what areas of expertise they drew upon, and what they intended their users to resist. I excluded two technologies because their methods of disconnection were not sufficiently distinct from the technologies that were chosen. A full list of the selected technologies can be found at Table 2-B below. Taken together, they represent the manufacture of disconnection in this thesis, but do not constitute the manufacture of disconnection as a whole.

\footnotetext{
${ }^{5}$ Max Stossel, the spokesperson for the Center of Humane Technology has not invented a technology of disconnection, and the Light Phone was not available or usable in New Zealand.
} 


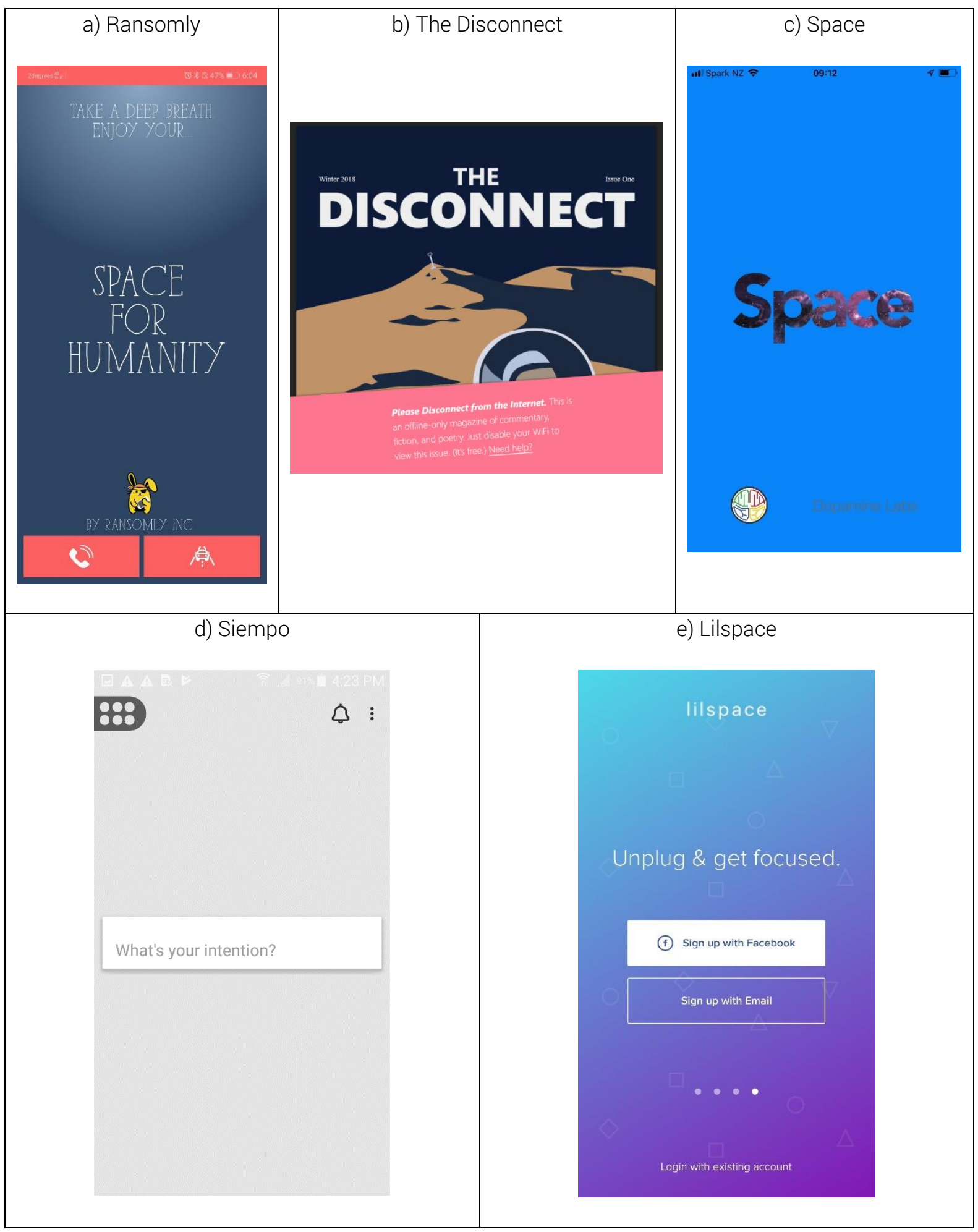

TABLE 2-B: THE HOME SCREEN OF EACH OF THE FIVE TECHNOLOGIES OF DISCONNECTION

\subsubsection{The walkthrough method}

In the next stage of the research process, I undertook a deep dive into the five selected technologies of disconnection. I used the walkthrough method to identify and 
critically assess the new technological relations with the internet that the technologies of disconnection offer. The walkthrough method was developed by Light, Burgess, and Duguay (2016) for humanities and social science researchers to critically interrogate software apps. Prior to the walkthrough method, studying apps in the humanities and social sciences was methodologically challenging. Many humanities scholars or social scientists are not software developers or computer scientists, and therefore lack the 'computational literacy' to analyse the technical components that constitute digital objects such as apps (Berry 2011). In addition, apps are proprietary software that provide limited access to technical components such as the underlying code, algorithms and backend architecture (Light, Burgess, and Duguay 2016). Some apps also resemble 'platforms' as closed and controlled systems, which are often commercially owned and organise activity within a specific software program (Plantin et al. 2016). The walkthrough method was conceived in response to calls for medium-specific methods (Rogers 2013) and builds upon app interface theory (Bratton 2015; Galloway 2012) and interface analysis methods (Stanfill 2014) ${ }^{6}$ to enable humanities and social science scholars without technical backgrounds to study apps in a way that complements critical analysis and social research agendas.

The walkthrough method takes its name from video game culture and design practice. Walkthroughs are a common genre in video games and software product reviews. Video game walkthroughs offer intricate details and step-by-step narratives of use to reveal hidden affordances or shortcuts about the game (Grimes 2015; Smith and Sanchez 2015). In design,

\footnotetext{
${ }^{6}$ App interfaces have been broadly theorised by media scholars to include backend architecture and other objects and actors beyond what the user can see (Bratton, 2015; Galloway, 2012). Stanfill's (2015) view of interfaces and new media as a productive power that produce and reinforce social logics, enables a stronger connection between the walkthrough and my theoretical Foucauldian framework. In particular, she implies that a method such as the walkthrough is a type of discursive interface analysis.
} 
the first walkthrough method was a Usability Inspection (UI) method in the field of humancomputer interaction $(\mathrm{HCl})$ called the cognitive walkthrough. The cognitive walkthrough method was developed by Lewis et al. (1990) to apply theoretical models from cognitive science to the practical design and evaluation of real-world technical systems. This model was meant to facilitate the easy learning of "walk-up-and-use" interfaces such as automated teller machines (ATM) by non-technical audiences, who could quickly learn the expected interaction if it were designed with certain principles, which Lewis et al. $(1990,193)$ call "design for successful guessing." The cognitive walkthrough has been iterated into many different versions in $\mathrm{HCl}$ and design such as the enhanced cognitive walkthrough, cognitive walkthrough with users or the cognitive walkthrough for the web (Mahatody, Sagar, and Kolski 2010). What all the versions of the cognitive walkthroughs have in common is an attempt to anticipate and incorporate user behaviours into the design process, with one of the latest iterations called the User Experience teardown (VikingCodeSchool 2020).

Light, Burgess, and Duguay (2016) differentiate their walkthrough as a critical method in comparison to the cognitive walkthrough from $\mathrm{HCl}$ and design. To enable the critical interrogation of an app, the walkthrough method draws upon the specific fields of science and technology studies (STS) and cultural studies. STS traditionally focuses on how scientific and engineering research and practices relate to wider sociocultural and political infrastructures. The walkthrough method specifically draws upon a theory within STS called actor-network theory (ANT) where humans (e.g., users and designers) and non-humans (e.g., design elements) are framed in relational terms and understood to shape each other and sociocultural phenomena (Latour 2005). Similarly, a core contention of cultural studies is that institutions, people and culture mutually shape each other. In the case of studying software, cultural studies offers a toolset for researchers to understand what wider cultural discourses 
an app both draws upon and reproduces. For example, the choice of a font, icon or background colour by a designer could be indicative of a wider dominant discourse about gender, sexuality, race or class. Both STS and cultural studies charge the walkthrough method with "analytical power" (Light, Burgess, and Duguay 2016, 882) as these fields invite researchers to interrogate how sociocultural phenomena are represented, reproduced and implicated by media technologies.

To identify the types of technological relations that the manufacture of disconnection produces, I systematically 'walked through' each technology of disconnection. I took screenshots and informal notes as I stepped through the installation and onboarding process of the app, as well as the features and functions in everyday use. All screenshots were coded, and electronic copies were kept in secure university cloud-based storage services. The notes considered how the technology of disconnection was supposed to be used or what Light, Burgess, and Duguay $(2016,883)$ call the app's "environment of expected use". Where video game or $\mathrm{HCl}$-based walkthroughs highlight the intentions and actions of the user or player, the walkthrough method shifts the point of inquiry to the intentions of the designer. In combination with data from my semi-structured interviews, I therefore looked beyond the app interface to wider contexts out of the user's control such as the disconnectionists' vision of the app, their businesses' operating model, and data governance practices such as how the app collected and handled users' data. In the case of this thesis, the 'environment of expected use' took on extra importance. As the function of a technology of disconnection is often to encourage non-use or change the users' behaviour, the app's environment of expected use translated into new digital architectures for the user to inhabit. Guided by my late Foucauldian theoretical framework, I interpreted the 'environment of expected use' as power relations beyond the interface that encouraged or forced the user to inhabit new types of mediated 
spaces or subject positions, that in turn, disconnect the user from connective opportunities within the attention economy. In the following five chapters of this thesis, I analyse these new technological relations in close detail, with each chapter focusing on one of the technologies of disconnection. While there was notable crossover of ideas and themes across each technology of disconnection, I determined that organising my analysis around one technology of disconnection per chapter would provide the clearest analysis and discussion of my research questions. In addition, the distinction in Foucault's work between technologies of space and self informs the overarching structure of my analysis. My case studies are divided into two groups: the production of disconnected space (Part II) and the production of the disconnected subject (Part III).

However, I found that the scope of the walkthrough method was too limited to analyse the manufacture of disconnection. The technologies of disconnection that I analysed were not limited to smartphone apps, but web apps too. I therefore supplemented the walkthrough method with a platform studies approach. Platform studies has a history in media studies and has been used to study the interactivity between platforms and web applications, and most commonly the accessibility of data and logic through software known as an application programming interfaces (APIs) (Helmond 2015). I adopted a platform studies approach to analyse how technologies of disconnection operate in environments other than smart devices. Combining both the walkthrough method and a platform studies approach enabled me to both describe and analyse the unique user experience and interactivities of technologies of disconnection that, in turn, imagine new opportunities and applications of digital technologies beyond connectivity.

By analysing how technologies enable or encourage disconnection, I hope to broaden methods to studying apps, platforms, and software. Recent application or platform studies 
approaches (Dieter et al. 2019; Helmond 2015) suggest network connections as entry points to studying software applications. I aim to reveal the opposite: the subject positions users are encouraged to inhabit to enable disconnective practices and the nuances in which network or social connections can be severed, delayed, or otherwise interfered with for the 'benefit' of the end user. My alternative perspective on software functionalities captures instances where people seek refuge from networks or even desire network infrastructure to temporarily fail. By doing so, I hope to complement the growing number of research projects that are challenging the connective bias in media studies. For example, Mannell (2018) identifies the affordances in messaging apps (such as the read receipt functionality) that implicate a users' obligation to respond, making it more difficult to disconnect from each other. I similarly hope to demonstrate that network disconnections offer an equally interesting and critical entry point to studying media technologies, platform and apps.

\subsubsection{Ethical and reflexive research considerations}

It was important to me that I considered ethical issues when designing the method and undertaking research for this thesis. Ethical approval to undertake the interview component of the study was sought and approved by the Victoria University of Wellington Human Ethics Committee (approval no. 25859). In designing the topic list, I considered a range of possible ethical implications and sent participants the set of topics in advance. I did not consider that the walkthrough component of my research raised any ethical issues; none of the technologies of disconnection have social components or involve other users, so I did not need to consider how my research activities impacted the privacy of other users. In 2018, I published an essay in The Disconnect, the technology of disconnection that I examine in chapter four. I approached Victoria University of Wellington's Human Ethics Committee for 
advice on whether writing for The Disconnect would constitute a conflict of interest which they concluded it would not.

I also considered how my own subjectivity influenced the approach of my thesis. Humanities research that is grounded in a Foucauldian or social constructionist epistemology acknowledges that the researcher's subjective experience shapes every aspect of the research process (Gergen and Gergen 2008), including how phenomena such as disconnection are interpreted. Braun, Clarke, and Gray (2017) suggest that personal factors shape researchers' choice of topic, methods, and the insights they develop; these personal factors offer useful analytic insights but also restrict what researchers see. Therefore, I identified the following aspects as particularly relevant to this project.

First, I approach this research as a disconnectionist, meaning I question constant connectivity and regularly explore new methods to disconnect from the internet. Nearly six years ago, I deleted my Facebook account because I felt that social media was negatively affecting my mental health. I learnt first-hand that there were benefits of being offline more often, as well as opportunity costs to spend time with friends and colleagues that I only kept in touch with via social media, and the general changes that social media has made to the social fabric of society. My experience of disconnecting influenced my decision to undertake a PhD on this topic and influenced my research process. I believe that sharing my personal journey of disconnecting from the internet made it easier for me to gain access to my interviewees, as well as acquire more meaningful data during my interviews.

I also actively advocate for others to disconnect from the internet. Throughout my PhD, I created and delivered a workshop for university students and staff on how to 
disconnect from the internet called Study Tech Habits. ${ }^{7}$ Over the course of developing and delivering this workshop, I tried to cultivate a critical perspective that emphasised that disconnecting from the internet was not a black and white exercise but could be a practical exercise for students, depending on their interests and needs. I worked with students to develop strategies for disconnecting from specific platforms or apps and at specific times, rather than encouraging outright disconnection. Similarly, my own relationship to social media and the attention economy has been nuanced. For example, I was never totally disconnected from social media or the attention economy, nor did I want to be. Throughout my PhD, I started a Twitter account to socialise with other PhD students and academics, wrote articles for the popular press, and marketed my own website and blog, requiring me to engage with the logics of the attention economy to publicise my research. My personal experiences have shaped my analysis of the professional and personal pressure on living in the attention economy.

Yet, I am aware that I have a particularly positive interpretation of the concept of 'disconnection'. As a self-identified introvert, I value the opportunities to distance myself from other people. I also experience the world from a place of privilege: I am a Pākehā (a New Zealander of European descent) middle-class man who lives in an affluent suburb in the outskirts of Wellington, New Zealand. I have regular access to green spaces that enable me to escape from the toil and turmoil of everyday life. I do not have any children nor social or cultural expectations that influence when or how often I can disconnect from other people (I critically discuss these themes more in chapter seven). I am in a long-term relationship which

\footnotetext{
${ }^{7}$ My workshop was originally called Healthy Tech Habits, but I changed the title to Study Tech Habits because I learnt that a moral title was off putting to potential workshop attendees. I discuss the moralisation of disconnection further in chapter three.
} 
means I rarely feel socially isolated or 'disconnected' in a pejorative sense. Due to my privileged identity, I can find safe and affirming social spaces both on and off the internet, compared to people occupying marginalised identities who may find more connection and meaning through online communities and social media (Gray 2009; Lucero 2017; Marino 2015). As such, I am in a position to see disconnective practices as positive because I do not stand to lose access to certain digital spaces and networks that may be more highly valued by other people.

Therefore, my interpretation of how technologies of disconnection reorganise relations to the internet may differ from someone who has a different relationship to the internet. I actively sought to consider how my subjectivity influenced my analysis by 'doing reflexivity' throughout the entire research process of my doctorate (Gough 2003). While conducting my field work, I noted how the interviews and technologies of disconnection made me feel and recorded this during the interview and walkthrough process. I also discussed and interrogated my analyses with my supervisors to ensure that my insights were grounded in the data (Gough 2003). While, overall, I believe my subjectivity drove and enriched my analysis, I was mindful to not become blinded by it. 
Part II

The production of disconnected space 


\section{Escaping the internet without having to leave it}

On the morning of Monday 21 September 2015, the residents of Alamo Square

encountered something unexpected. A new regulation had seemingly appeared overnight in the central San Francisco neighbourhood. Affixed to a concrete pole on the corner of Alamo Square was an official-looking sign that warned park goers that they were entering a "No Tech-Zone" (Figure 3-A). Adopting a similar typography and authoritative tone as existing municipal parking protocols, the No Tech-Zone sign stated: "NO CELL PHONES, TABLETS, LAPTOPS, OR SMART DEVICES PERMITTED. VIOLATERS SUBJECT TO \$300 FINE." Early reactions from residents were mixed, with some suggesting local park officials had lost their mind while others claimed the sign reminded them to not become "slaves to technology" (qtd. in Mayton 2015). The San Francisco recreation and parks department responded quickly that the command did not come from them and took down the sign the following day.

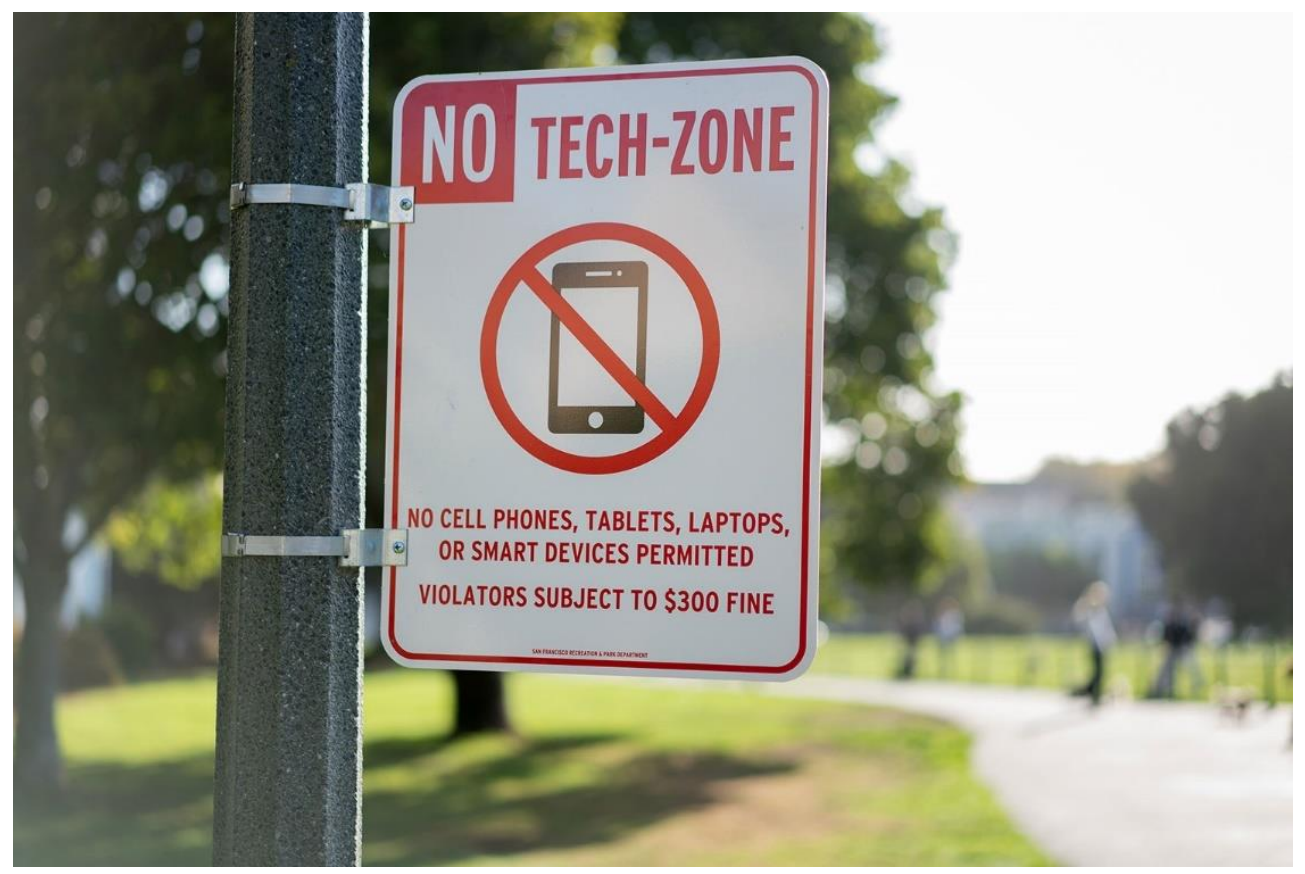


Eventually, it was revealed that the No Tech-Zone signs were an act of protest. A month later, further No Tech-Zone signs appeared throughout San Francisco city, including Duboce, Dolores and Washington parks. Local technology artist Ivan Cash stepped forward as the provocateur behind the signs. When interviewed about his motivation for the project, Cash identified as a former resident of Alamo Square who could not help but notice the number of tourists and residents who spent the majority of their time in parks glued to their screens. "We're just consumed [by technology]" claimed Cash (qtd. in KTVU 2015). Cash thought an alternative version of public parks where digital technologies were prohibited could be an interesting thought experiment. Cash admitted he did not endorse such an authoritative ban of smartphones, but rather saw merit in the idea to provoke debate about the influence of business-driven connectivity in the use and feel of public spaces. In a short autoethnographic documentary about the No Tech-Zone project, viewers learn that Google funding has enabled Wi-Fi to be freely available in San Francisco public parks (Cash 2015). A line of white text shortly follows in the documentary that questions: "Aren't parks supposed to be a natural escape from the modern world?" The insinuation from Cash and the No TechZones project is that public parks are no longer an escape from the modern world because Google-funded Wi-Fi has enveloped them.

This chapter explores how technology and a different use of space can enable users to escape the internet. I analyse a smartphone app and beacon technology called Ransomly that, similar to the No Tech-Zone project, both problematises and presents a different use of space in relation to digital technology. By simulating a ban on technology devices in a public space, the activism of the No Tech-Zone project is simultaneously anti-big technology platforms and moralistic. I investigate in this chapter how these seemingly strange bedfellows come together to co-constitute a rehabilitative type space that provides a 
convenient escape from the attention economy. However, I first establish that disconnection and media scholars have tended to analyse disconnected space in binary terms and spatial extremities. I begin the chapter by observing how disconnected spaces have been positioned at the margins of society, either as a type of infrastructural impoverishment or a luxury commodity that only the wealthy can afford. In contrast to these spatial extremities is Ransomly, an internet jamming beacon that works with smartphones to create an internet dead zone in a location of one's choice. I argue that Ransomly is a technology that manufactures a disconnected space to escape the internet. According to Foucault (1993), "space is fundamental in any exercise of power", and in this chapter I adopt Foucault's concept of heterotopia, or other space, to argue that Ransomlycreates a type of rehabilitative space that is the 'other' of ubiquitous connectivity. Inside this disconnected space, inhabitants can resist dominant communicative norms and cultivate alternative moralistic values in relation to digital media technology use.

\subsection{Binary views on disconnected spaces}

From Plato's ([340 BCE] 2005) famous declaration that the technology of writing would ruin his capacity to memorise, to Henry Thoreau's ([1854] 1995) musings on the merits of isolation in Walden, why people disconnect has a rich literary history. As a modern cultural practice in response to the proliferation of digital or information communication technologies (ICTs), however, academic discourse on disconnection is at a relatively nascent stage. Yet in the short space of the 1990s to the 2010s, the rhetoric of disconnection has changed significantly. I discuss the shift in disconnection discourse in the following section. 


\subsubsection{Disconnection as a dystopia}

For the majority of internet history, a disconnected space has largely been viewed as dystopic. A dystopia is a literary device most commonly used in the genre of science and speculative fiction to imagine a place that is worse than the one we currently live in (Moylan and Baccolini 2013). In urban studies a dystopia has been discussed as parts of a city that are viewed as "physically proximate but institutionally estranged" from other spaces (Macleod and Ward 2002, 154). Similarly, media and information studies have viewed certain areas and spaces that lack internet infrastructure as dystopic because they are considered information poor (Black 1986; Doctor 1991; Krieg 1995; Wilson 1987). For the best part of the 1990s and 2000s, discussions of disconnection were primarily about people in particular locations and demographics lacking the ability to connect. Disconnecting from the internet was framed negatively, in opposition to technology use. These discussions first appeared in texts where the use of loaded terms such as "information apartheid" (Krieg 1995) or "information poor" (Black 1986; Doctor 1991; Wilson 1987) framed non-use as impoverishment or on the wrong side of the digital divide (Wills 1999). The 'digital divide' refers to the "gap between populations that have easy access to ICTs and those who remain underserved" (Flew 2014, 23) by them. Disconnection was therefore something that governments, technology companies, and social enterprises have instrumentalised in creating the conditions for evergreater connectivity.

In the late 1990s and early 2000s, scholars and policy makers focused their attention on the global diffusion of ICT access (see 6 P and Jupp 2001; Bolt and Crawford 2000; OECD 2001). As the volume of this work grew during the mid-2000s, data increasingly emerged that demonstrated the original 'digital divide' metaphor focused on issues of access in ways that were overly simplistic (Murdock and Golding 2004; Norris 2001; Selwyn 2004; Servon 2002; 
Warschauer 2004). In his critical assessment of divides research, Niall Selwyn $(2003,106)$ criticises early accounts of the digital divide for having an ideological and deterministic view on technology:

[T]he whole notion of the information society is based upon an ideological belief in the positive and socially integrating power of technology alongside a prevailing ethic of instrumental rationality and strategically practiced self-interest towards accruing such benefits... [F]rom this perspective to not use [technology] is to choose not to be part of the information society -an irrational and ultimately disadvantageous position to adopt.

The techno-deterministic position critiqued by Selwyn - that a mere connection to the network or access to the information society is transformative-is further commented on by Michelle Rodino-Colocino (2006). Similar to Jodi Dean's (2005) observation that the commodification of communication fetishises technology, Rodino-Colocino argues that a techno-deterministic perspective recasts the digital divide as a problem of technological diffusion, conveniently serving the needs of the telecommunication sector instead of addressing endemic structural inequalities. While the scope of digital divides research has widened, technophilia continues to pervade subsequent strands (Hargittai 2007, 2012; Neves and Mead 2018), reinforcing a softer version of techno-determinism. The belief in empowerment through technology is a form of cultural bias; in a study on technophobia, Bauer $(1995,109)$ contends the notion that non-use of technology is abnormal or a deficit to overcome is culturally Western in origin, reflecting a "Californian way of life".

An issue with the dystopic lens on disconnection is that it misses the various motivations for why people may not wish to use the internet. A number of empirical studies have sought to understand the various social, cultural and economic reasons for disconnection. These categories include resistance, rejection, exclusion or expulsion (Wyatt, 
Thomas, and Terranova 2002), lapse or rarity of use (Selwyn 2006); institutional distrust (Mainwaring, Chang, and Anderson 2004); socio-economic displacement, disenchantment or disinterest (Satchell and Dourish 2009); or being forced, reluctant, selective or partial in use (Wyatt 2014). Analysing a selection of these studies, Hesselberth $(2017,1997)$ considers reasons for non-use to often be situational, including "peer pressure, negative stigmatization, technology fatigue, the network scale of effect, a sense of (dis)empowerment, the need for socio-economic security, or the distress over violating personal or professional relationships." Approaches that view disconnection as dystopic overlook the many nuances that underline why individuals may choose to reject or disengage with technologies.

Furthermore, non-use has not just been categorised as a passive state, but also conceived as a process or conscious mode of departure from media technologies. Scholars have offered several terms to conceptualise this idea, encapsulating a wide range of scenarios where, when and why such disconnection takes place. In a study on Facebook 'quitters', Portwood-Stacer (2012c) conceives of 'media refusal' to capture acts of conspicuous non-consumption which manifest as backlash to a specific platform, company or practice. Woodstock (2014) interviews a collection of 'media resisters' who unplug due to their perception of the 'inauthenticity' of digital communication, while Foot (2014) adopts the term 'media pushback' to describe non-use in employment or organisational settings, citing a lack of productivity or concerns about data abuse or security as reasons. Another motive for departure is the slippage between work and leisure and the burden of constant availability that can lead to information overload or work burnout (Ames 2013; Baron 2011; Mazmanian 2013).

Viewing disconnection as a state of impoverishment also derives from a flawed Western philosophical tradition. Much of the early rhetoric painted the internet as clearly 
distinct from the 'real life', erroneously positioning the internet as a 'parallel world' into which, with ICT access, people could simply leap and immediately begin reaping the benefits of a networked society. There are limitations to compartmentalising the communicative states of non/use, online/offline, and dis/connection into binary and rigid terms. Jurgenson (2012) identifies this categorisation to be evidence of digital dualism: a Cartesian perspective that assumes physical and digital space are experienced distinctly. Jurgenson's criticism is visualised below in Figure 3-B.
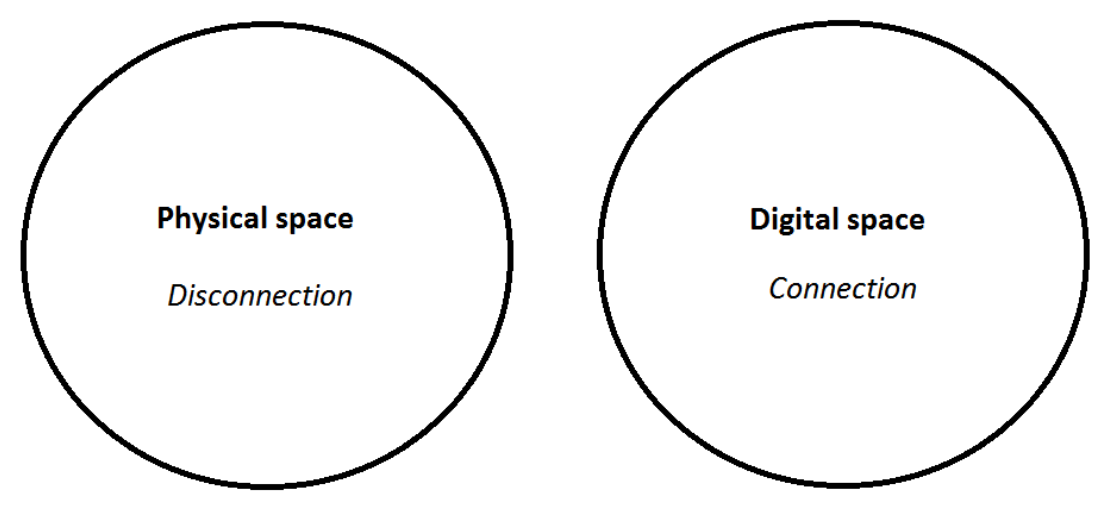

FIGURE 3-B: CARTESIAN DISCONNECTION

The insistence on a rigid distinction between physical and digital space frames disconnection as limited to physical space, which lends itself to a simplistic position that disconnection can only be undertaken outside the reach of mediation. Unmediated disconnection is an overly generalised conceptualisation that misses the many practices of disconnection with technologies, such as unfriending someone on a social media platform or separating Spotify and Facebook accounts to maintain a level of privacy (Light 2014). Furthermore, a Cartesian reduction of disconnection lends itself to the technophobic view that digital cultural practices are unhealthy, which has increased the escapism of disconnection, and is explored in the next section. 


\section{1.2. Disconnection as a utopia}

Predating dystopias are utopias. The term utopia was coined by Sir Thomas More ([1512] 2020) in the early sixteenth century and derives from two Greek words: eutopia (good place) and outopia (no place). More's Utopia tells the story of a voyager who stumbles upon the Island of Utopia, which is based on an ideal political constitution with laws and social customs that were far removed from the reality of $16^{\text {th }}$ century England. More's idea of an ideal constitution influenced subsequent understandings of utopias, including Foucault's, who viewed utopias as an idealised conception of society that exist outside of power relations and therefore are not located in reality (Hetherington 1997; Peters and Humes 2003). Escaping day-to-day reality aptly captures how popular and scholarly attitudes towards disconnection have changed. Where once disconnected locations (and the people in them) were considered impoverished or on the wrong side of the 'digital divide' (Wills 1999), locations without digital infrastructure, such as a mobile or Wi-Fi connection, are now considered opportune spaces for concentrated work or luxury spaces to enact various forms of disconnective practice that reignite people's awareness of their physical presence (see Plaut 2014, 2015). For example, there are reports that Silicon Valley executives send their children to expensive technology-free Waldorf schools (Jenkin 2015) so the children can learn without distractions. Within an economic and social system that has made privacy itself a luxury commodity (Papacharissi 2010), rather than being associated with scarcity, disconnected environments - at least for those with the cultural and economic capital to move in and out of such spaces freely-are now perceived as exclusive leisure and relaxation zones. Most urban areas offer little digital silence, whereas the dark spots of the internet where there is no mobile or Wi-Fi signal provide opportunities for people for concentrated work or to ground themselves in their surroundings and become aware of their physical 
presence (Plaut 2014). A sense of disconnection from a physical space because of the distraction of digital devices has encouraged people to disconnect from the internet (Syvertsen and Enli 2019).

Some spaces of disconnection have become luxurious because escaping the internet is increasingly difficult to undertake in developed countries. In many urban parts of the world, internet access has become ubiquitous to support the increasing day-to-day activities that require an active connection to the internet. A consequence, however, is that an easily accessible internet has turned the internet into something that is routine or mundane. A 2017 viral tweet from Twitter user @Noahpinion (April 29, 2017) succinctly summed up this shift: "15 years ago, the internet was an escape from the real world. Now, the real world is an escape from the internet." Digital detox summer retreats in the United States can cost up to 700 USD per person (Fish 2017; Sutton 2017). One example of a digital detox retreat in Australia is Unyoked(2019), which offers customers a "private cabin in the wilderness". In the frequently asked questions section of the website, readers are told: "We've designed Unyoked to help you get off the grid when you need it, think cabin porn IRL [in real life], your own tiny house in the wilderness. Each of our hideouts are tucked in their own secluded area of unique wilderness we've unlocked just for you." The cabin IRL offered by Unyoked is the type of disconnection that I argued some scholars advocate for in chapter one. Unyoked is reminiscent of Hesselberth's $(2017,1995)$ conceptualisation of an unmediated disconnectivity as "the tendency toward voluntary psychic, socio-economic, and/or political withdrawal from mediated forms of connectivity". Kuntsman and Miyake (2019) similarly call for scholarship on escaping media, as opposed to naturalising the presence of technology in day-to-day life. 
The belief that a limit to connective culture can be found by focusing on unmediated forms of connectivity is emblematic of Cartesian or digital dualism that rejects digital mediated space. The Cartesian perspective is utopian and problematic for a number of reasons. The first is that this view assumes mediation to be synonymous with digital technology and misses a historical perspective of media relationship(s) with social, cultural and economic practices. Offline advocates are the subject of David Banks' (2015) etymological enquiry into 'online' with a focus on the railroad history of 'on-the-line' and 'offthe-line' states. Banks rejects the online/offline dichotomy in favour of viewing online/offline as a continuous negotiation of social relationships. He argues that off-line states are "not necessarily about a total removal or separation from a network, but rather a configuring of relationships such that the network's influence on the subject is weakened." An example is the standardisation of time zones. As all individuals are impacted by time zones, one cannot truly be 'offline'. Similarly, while anyone can switch off their Wi-Fi router or turn their phone into Airplane mode, a type of signal jamming to block the transmission of communication signals (Mannell 2018), they cannot opt-out of the social and communicative norms that mobile and ubiquitous media have changed. Banks' work supports a similar argument by Satchell and Dourish (2009), who consider that distinctions between technology use and nonuse are misleading because all communication is fragmentary. It may be that ubiquitous technology is speeding up the convergence of on/offline and dis/connection. Floridi (2014) offers the neologism 'onlife' to describe the proliferation of cloud computing, mobile networks, the Internet of Everything (loE), and sensor technologies that are blurring distinctions between online/offline. Locative media is another subfield of media studies that demonstrates the hybridisation of communication, information and media onto physical space (see Farman 2011; Frith 2015). By focusing on binary distinctions between 
non/mediation, Hesselberth mischaracterises physical spaces as free from mediation or influence, missing the ways that all space is subtly shaped by networks and digital technologies.

Unmediated disconnection is also a utopian fiction of living, actively informing imaginations of disconnection but divorced from day-to-day practice. The dream of escaping to a remote, disconnected part of the world is, at best, an ideal for many people because spaces and social relations are not just mediated, but mediatised, meaning intertwined and interdependent with media (Hjavard 2013). Hjavard $(2008,113)$ describes the mediatisation of society as a process where "the media have become integrated into the operations of other social institutions, while they also have acquired the status of social institutions in their own right." Everyday communication, information search, events management, transport, shopping, banking are a few examples of activities and transactions that have been digitised and intertwined with media technologies. The cost of not using technology is significant and increasingly unfeasible because digital platform technologies have become essential infrastructure to day-to-day practices (Couldry and Hepp 2017; Plantin et al. 2016).

Our dependency on technology was tested in a study on forced disconnection by Kaun and Schwarzenegger (2014), who required participants to abstain from media use for seven days. Only a small percentage remained abstinent for the full period, with some claiming their relationships or livelihoods depended on a connection. These results are supported in a similar study by Roberts and Koliska (2014) on the effect of ambient media. In finding that participants could not abstain from media use, they emphasised the degree to which human culture is intertwined with media technologies. In all, the view that disconnected spaces as either dystopian or utopian precludes an understanding of how escaping the internet is being integrated into everyday spaces. 


\subsection{The heterotopia of Ransomly}

There is an alternative way to theorise disconnected space. In contrast to dystopias and utopias are heterotopias. A heterotopia is an 'other place' or 'counter-site' and means a different ordering of language, things or spaces (Foucault 1986). According to Helen Sohn (2008), Foucault derived the notion of heterotopia from medicine, where it refers to normal tissue in an abnormal place in the body. Compared to Foucault's writings on power/knowledge or technologies of the self, heterotopia is a minor concept within his work, but one that has influenced spatial theory across a number of disciplines, including geography, architecture and urban studies. The concept appears three times within Foucault's work: in the preface to The Order of Things ([1966] 2002); in Of Other Spaces (1986); and in a radio recording Le Corps Utopique, Les Hétérotopies ([1 966] 2009). Geographer Edward Soja $(1996,162)$ famously described Foucault's heterotopias as "frustratingly incomplete, inconsistent, [and] incoherent." Perhaps the ambiguity of Foucault's heterotopia explains why a broad variety of spaces have been conceptualised as one, including cyberspace (Chun 2006); Facebook (Rymarczuk and Derksen 2014); therapeutic spaces (Honig 2017; Vakoch 1998) and political protests or social movements (Beckett, Bagguley, and Campbell 2017), to name a few. While the concept of heterotopia has lost some of its analytic purchase by being applied broadly (Young 1998), I believe it is a useful concept to capture alternative spatial formations that do not fall neatly into binary categories (e.g., private/public or on/offline) and challenge routine living in the attention economy.

A technology of disconnection that simulates the experience of escaping the internet is Ransomly, an app for smartphones with Android operating systems that utilises Bluetooth technology and a beacon hardware device to create a disconnected space in one's location of 
their choosing. In the next sections, I draw upon Foucault's heterotopia and the walkthrough method to analyse Ransomly, arguing the technology of disconnection produces a digital architecture that functions as an 'other' to connectivity. My walkthrough and analysis of Ransomlyas a heterotopia is organised into three discrete aspects of using the product: accessing the disconnected space via the onboarding process of opt-in, inhabiting the rehabilitative space, and the opportunity to contest communicative norms once inside the disconnected space.

\subsection{The convenience of opt in/out}

Ransomly manufactures a type of disconnected space by interfering with the connectivity of a smartphone in a location of one's choosing. The process to create a disconnected space via Ransomly begins with obtaining a beacon, which can be purchased from the Ransomly website for the cost of 40 USD. Once users have obtained a beacon, they must download the Ransomlyapp and complete an onboarding process that enables certain software protocols to restrict how they can use their phone. When opening Ransomlyfor the first time, users are guided to an onboarding process (see Figure 3-C). The tutorial explains to the user that disconnected space depends on the location of the beacon and user. Users must agree to three permissions: Do Not Disturb, apps that appear on top, and location. Prager explains to me how the disconnected space works:

\footnotetext{
You have this Bluetooth low energy where you can have iOS and Android phone's listen for signals all the time without compromising battery performance too much. It takes some energy. On the other side there's these things called beacons, and these emit Bluetooth signals and these have packet information in them. You can have your phone listening for Bluetooth signals like radio signals. It gets one and if it has a packet that it understands like our app is listening for a certain type of Bluetooth signal, then it can achieve some software methods in that app.
} 
Enabling these permissions enables Ransomlyto recognise the location of the smartphone in relation to the beacon, and automatically turn on the Do Not Disturb functionality and a block screen when the user comes within ten feet of the beacon. A key requirement of Ransomly is that the disconnection must be bottom-up driven, meaning consent is provided by the user for the block to work. The requirement of consent is heterotopic; according to Foucault (1986, 26), a principle of heterotopias is that they are demarcated by a system of opening and closing "that both isolates them and makes them penetrable" (emphasis added). What Foucault means is that the accessibility of a heterotopia for inhabitants is tied to certain rules in relation to the function of the space. Rymarczuk and Derksen (2014) comment further that permission to access a heterotopia is only granted when "certain acts like purification, identification, registration, payment, demonstration or worship" are performed. In the case of Ransomly, the onboarding process functions to enable Ransomly to work as well as obtain user consent to allow future restrictions to be placed on the user's smartphone. In the absence of an onboarding process and provision of consent, Ransomly would be indistinguishable from top-down enforced disconnection, such as the internet kill-switch that the Egyptian government under the Hosni Mubarak regime allegedly developed to censor its citizens (Gerbaudo 2013). 

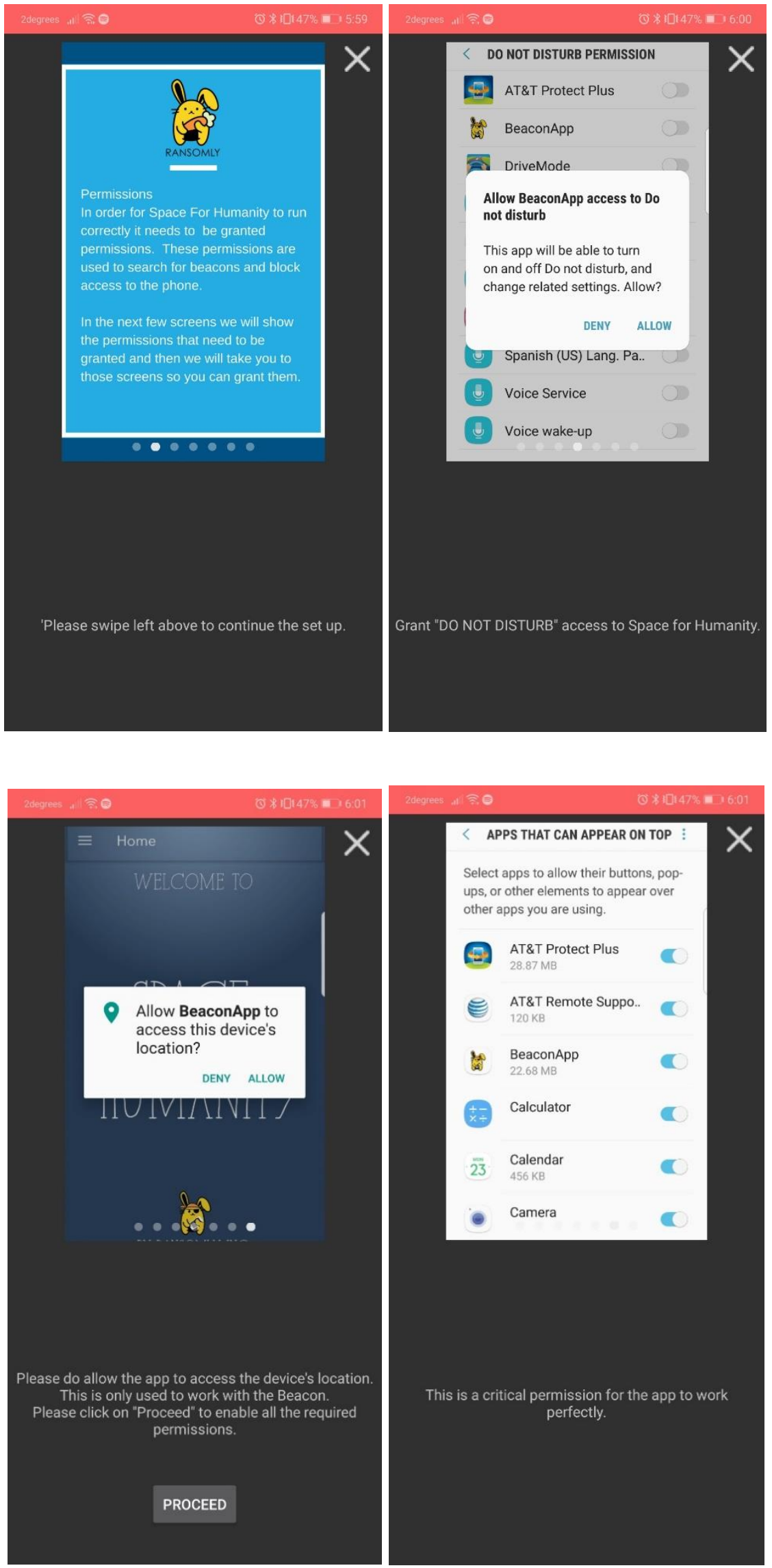

FIGURE 3-C: PERMISSIONS THAT ENABLE RANSOMLY TO WORK 
The opt out/in requirements of Ransom/yalso make a disconnected space more penetrable. Once the user has completed onboarding and placed the beacon in their desired location, the default status of the smartphone within ten feet of the beacon changes from connected to disconnected. The designation of a space that is disconnected-by-default provides a key mechanism to make disconnecting from the internet easier. I do not mean to suggest that disconnecting is always very difficult to achieve or inaccessible, but more that the 'smart' urban environment is designed to favour connection. As discussed earlier in this chapter, an increasing number of activities and transactions are mediatised, with connectivity increasingly the default setting and disconnecting being an opt-out exercise. Thanks to a proliferation of mobile devices, Wi-Fi and mobile networks, the default setting for mobile phone users in most urban locations is a connection to the network. An increasing number of digital services (e.g., banking, security two-step verification, public parking, to name a few) require an active connection to the network, and rewards and other psychological incentives are frequently deployed or embedded in the environment to encourage connective user behaviour (McCullough 2015). Despite the pro-connective environment, users do have relatively useful tools for disconnection at their disposal. A well-known mobile phone feature that mutes notifications is Do Not Disturb, first appearing in the Apple Mountain Lion operating system (OS) X and iOS 6 in 2012, with later versions offered in iOS and Android OS (Plaut 2015). Airplane Mode is another favoured tool to disconnect with (Lee et al. 2014). Both Do Not Disturb and Airplane Mode are features that enable disconnection for smartphone users but to a large extent require opt-in or an active decision to work.

A discipline that understands the significance between opt-in and opt-out is choice architecture. Choice architecture is a practice and subfield of behavioural economics that uses 'nudges' or subtle psychology to enhance public policy outcomes in Western 
democracies (Thaler and Sunstein 2009). Choice architects cite evidence of the 'default effect', which contends that people are generally willing to follow the path of least resistance, suggesting default options will likely be chosen (Thaler and Sunstein 2009). In a famous study on organ donation schemes, Johnson and Goldstein (2003) concluded that people are far more likely to consent to donating their organs if the question is designed as opt-out as opposed to opt-in. Sunstein (2014) labels the default effect and other nudges as 'soft paternalism' to acknowledge the potential influence of the design on user behaviour while avoiding foreclosing the opportunity for the user to choose against the designer's wishes. The effect has been used to optimise built environments such as supermarkets (Kroese, Marchiori, and de Ridder 2015; Lindstrom 2011) and online checkout interfaces to encourage consumer activity.

Regulators have recognised the subtle power of the default option to steer online user behaviour. One of the key changes brought by the European Union's (EU) General Data Protection Regulation (GDPR) privacy law was raising the standard of online customer consent to require a positive opt-in (van Ooijen and Vrabec 2019). The EU interpreted the default effect as leaving online users vulnerable to commercial persuasion. The GDPR law change more broadly suggests that any disconnective features that require active choice such as Do Not Disturb or Airplane Mode present small but significant psychological barriers to disconnecting from the internet. Offloading choice from consenting individuals to designers on their behalf is increasingly common in health policies and programmes. One such example is the Blue Zone Project, a health initiative that takes its name and inspiration from regions in the world (e.g., Okinawa, Japan or Sardinia, Italy) identified as 'blue' for having the highest concentration of centenarians in the world (Carter 2015). In a study on Blue Zone communities in the United States that attempt to emulate Blue Zone regions of the world by 
redesigning the built environment, Carter (2015) observes that shifting the burden of choice from the individual to the built environment lowered psychological barriers to healthy outcomes. The shift in individual agency to the environment means that the political rationality of nudges differs somewhat from neoliberalism, meaning that the individual is not assumed to make decisions in their best interests (Jones, Pykett, and Whitehead 2013, 2011). Prager is similarly motivated to use the physical environment to encourage users to disconnect from their devices. During our interview in the Gaslamp Quarter of San Diego, he explains his interest in the physical environment as an agent of behavioural change by gesturing towards the wider street:

This environment is clearly made for driving which gets interrupted all the time by noisy agents, it's terrible, or at least terrible to me. But you could redesign this whole Gaslamp district where you had to walk around it right? And make it interesting to walk around it.

Ransomly reduces the psychological barriers to disconnecting by creating a specific space for disconnection, in which the default setting is disconnection. In other words, the transformative potential of a disconnected space is the contribution of an actual location towards the process of disconnecting. A useful analogy that Prager provides to me is an onoff switch. People have on-off switches for infrastructure such as electricity or water to make necessary disconnections. If people did not have taps or switches to modulate the flow of water or electricity, they would flood their house or waste electricity. Prager explains to me that he views an on-off switch as a behavioural tool:

[Imagine] an on and off switch right, like a carrot or stick. And that becomes an application. It's like well we can switch off [smartphone] applications programmatically, so we can use that as motivators to keep people to stick to things that are good for them. That's basically the birth of Ransomly. It's the very stripped-down version of like I don't know what you want to block [your smartphone] for but I'm going to allow you to block it basically. 
Similarly, internet kill-switches such as Play Limit and Token Time offer parents a convenient means to set guidelines with their children's media use (Plaut 2015) and control the flow of information within their home. Yet Play Limit and Token Time both offer disconnection around segments of time. A space of disconnection is representative of an on-off switch to the internet in the form of a disconnected space. Entering and exiting the area of disconnection is the equivalent of flicking an on-off switch to the internet. Once the user of Ransomlyenters the space, no active or ongoing choices are required to disconnect other than to leave the space. Disconnecting becomes easier because it is the default option in the designated space. Similar to a quiet coach on a train or a study room in a library, Ransomly assigns a function of limited communicative practices to a designated space.

\subsubsection{A space for rehab from smartphones}

As soon as all permissions are enabled and the user enters the designated space, a block is in effect via the Bluetooth beacon, and a block screen appears. The software methods in the Ransomly app lock the phone from being able to be used for any purpose. The block screen cannot be removed or shut down, rendering any other functionalities other the smartphone practically unusable. The block screen informs the user to "take a deep breath [and to] enjoy [their] space for humanity' (see Figure 3-D). The idea of calling a disconnected space a 'space for humanity' is informed by Prager's background in positive computing, a practice that combines positive psychology and software development to create "technology to support psychological wellbeing and human development" (Calvo and Peters 2014, 2). The message to the user is that they have entered a space that is beneficial for their wellbeing. 


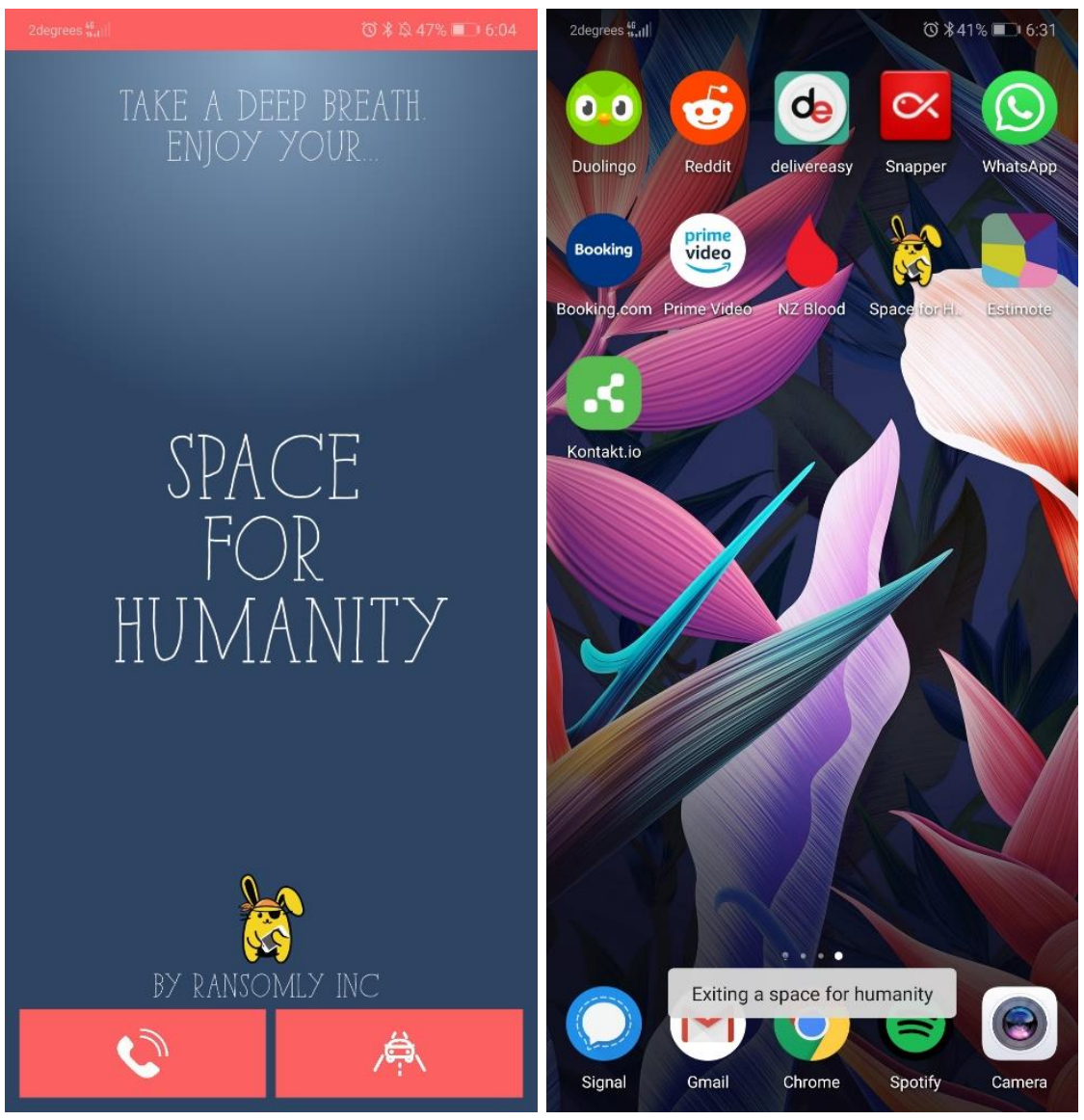

FIGURE 3-D: RANSOMLYBLOCK AND EXIT SCREEN

For a disconnected space to serve human development or wellbeing requires an imagination of what digital space and dis/connections can function for. Foucault saw heterotopias as vehicles for imaginaries of alternative versions of the good life which, in the context of digital disconnection, could be a convenient way to escape from the internet. Foucault $(1986,24)$ contended that heterotopias integrate the imaginary into social reality and existed in all cultures:

\footnotetext{
There are also, probably in every culture, in every civilization, real places- places that do exist and that are formed in the very founding of society-which are something like counter-sites, a kind of effectively enacted utopia in which the real sites, all the other real sites that can be found within the culture, are simultaneously represented, contested, and inverted.
} 
To provide historical examples of counter-sites, Foucault distinguished between heterotopias of crisis and deviance. Heterotopias of crisis were common in premodern societies where pregnant women or the sick were taken to partake in rituals and away from the rest of society. Heterotopias of deviance were modern institutions_-prisons, schools, mental asylums — where the non-productive and 'abnormal' were housed and kept separate from society (Foucault 1986). Ransomly is a heterotopia of deviance that acts as a counter-site to the types of hyper-connected spaces produced by the attention economy. In the first chapter, I noted that the Silicon Valley disconnectionists have concerns that their designs and technology platforms are driving an attentional crisis for users of digital technology. Having concerns about an attention crisis in turn creates ideas of 'deviant' or 'abnormal' smartphone usage in relation to time spent on device. ${ }^{1}$ Prager is a Silicon Valley disconnectionist who tells me the purpose of Ransomly is to counter the attention economy and "optimise for wellbeing rather than just attention." He sees Ransomly and the restrictions imposed on users inside the disconnected space functioning as a type of rehabilitative space:

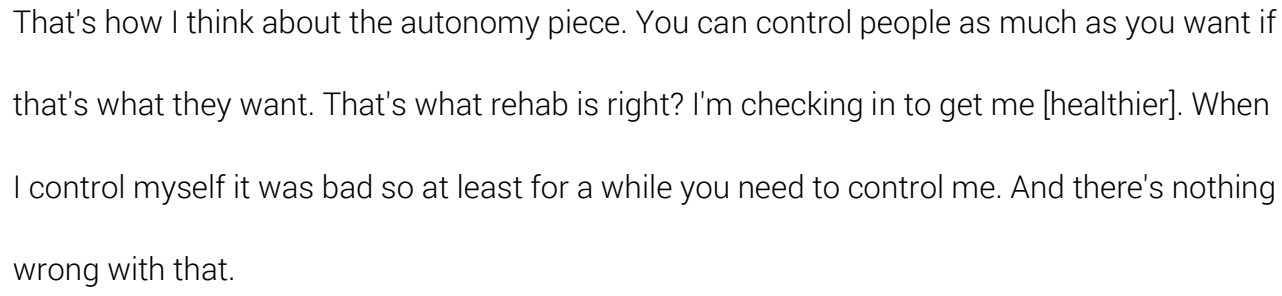

As a heterotopia of deviance, Ransomly functions as a therapeutic space for users who feel addicted to their smartphone and seek rehabilitation from it. Spatially isolating a user from their internet connections is justified on the basis that excessive smartphone behaviour is abnormal and requires treatment. A disconnected space functions similarly to psychoanalytic therapy sessions that, according to Vakoch $(1998,355)$, are heterotopic because they allow

\footnotetext{
${ }^{1}$ I critique the moralisation of disconnection in the final section of this chapter.
} 
"patients to experience and conceptualise things differently because they provide a real space into which the imaginary can be introduced." Ransomly is a therapeutic space that integrates the imaginary of escaping the internet with the reality of day-to-day living.

Viewing Ransomlyas a therapeutic space for deviant smartphone users is reinforced by some of the iconography of the app interface (see Figure 3-D). A cartoon yellow bunny wearing a bandanna and eyepatch and holding a smartphone occupies the lower part of the Ransom/y block screen. The cute aesthetics of the rabbit suggests the disconnected space produced by Ransomly is meant to be a space of play, perhaps for children and their families. In my interview with Prager I learn that his ideal target market of Ransomlyare parents, particularly those with young children who desire to raise their children in a mediated environment that was closer to their own. Parents in their thirties and upwards can recall a time before the internet, or of dial-up internet that predated Wi-Fi and access to the internet was localised to a specific part of the house. On the Ransomly (2020) website, users are told they can use Ransomly to "reclaim the dinner table", positioning certain family rituals like mealtimes as being co-opted by the attention economy and the culture of connectivity. In a disconnected space like the one provided by Ransomly, parents can 'hold the phone at ransom' to re-establish spatial boundaries that existed before internet access became mobile. As the beacon can be placed anywhere the user wishes, a parent could use Ransomly to help enforce phone-free spaces such as a phone-free bedroom and address concerns that technology devices are interfering with the parenting of their children (McDaniel and Coyne 2016). 


\subsubsection{Contesting dominant communicative norms}

Another principle of heterotopias is their relation to other spaces. Foucault $(1986,24)$ explains that heterotopias "have the curious property of being in relation with all the other sites, but in such a way as to suspect, neutralise, or invert the set of relations that they happen to designate, mirror or reflect." Ransom/y provides a direct contrast to connected spaces where people can use their smartphones. A disconnected space 'for humanity' provides a contrast to the connected spaces around it, implying such connected spaces are, at times, 'inhumane'. Thanks to mobile phones and social media, people with smartphones can be 'negatively' affected by the internet regardless of their location. The advent of social media has changed communication and interaction patterns: if social relations were previously confined to our physical location, now we can be immersed in multiple digital worlds interacting with many individuals regardless of our physical location and distance from each other (Couldry and Hepp 2017, 90). Network mobility has, in turn, opened the floodgates for affects, feelings, and emotions (or "affect-laden practices") to flow relatively unconstrained, forming discursive and emotive patterns not confined by the divisions of the physical world but characterised more appropriately as "displays and flows of varied intentionality and intensity" (Giaxoglou and Döveling 2018, 2). As a result, however, new pressures emerge as individuals are expected "to be available for interaction on digital media platforms" and simultaneously pressurised to "represent itself on these platforms" (Couldry and Hepp 2017, 145).

It is when the block is functioning and the user is inside the disconnected space that Ransomly calls into question the 'reality' of the networked space around it. Boyer $(2008,54)$ argues that "heterotopian spaces operate via a double logic: they are real spaces that show reality to be the illusion, or they are perfected spaces, more rational and ordered than normal 
spaces." Heterotopias like Ransomly act like mirrors by offering a counter-normative function, a point of difference to contest spatial norms. An example of a spatial norm is moving in the 'right way' in public spaces (Beckett, Bagguley, and Campbell 2017) such as obeying pedestrian bylaws, or the 'appropriate' actions or activities for a given space, such as not using a smartphone while in conversation or in public. The degree to which new technologies reconfigure inhabitants' presence is a long-standing debate in media studies. Numerous scholars contended media communication technologies disrupt inhabitants' sense of place. Meyrowitz (1985) famously argued that television and other electronic media disrupt the social behaviour of viewers. On a similar note, Gergen (2002) blames mobile technologies for creating 'absent presence'-being physically somewhere but mentally or cognitively elsewhere; while Ling (2008) argues mobile connectivity draws a user's attention away from their immediate environment. ${ }^{2}$ Recent terms such as 'phubbing', a portmanteau of phone and snubbing, and 'smombie', a combination of smartphone and zombie, suggest that concerns about absent presence have trickled into popular discourse. Public policy has even responded to absence presence. In the German town Augsburg, pedestrian traffic lights have been installed in the ground to help smartphone users cross the road safely (McGoogan 2016). Ransomlyattempts to question such norms by providing an alternative taxonomy of spatial norms and use of space. Young (1998, para. 22) writes:

\footnotetext{
${ }^{2}$ It is important to note that the absence of presence in physical spaces has been refuted by several media scholars. As I have previously mentioned, there is Jurgenson's (2012) 'digital dualism', where digital activities are viewed as problematic and offline activities exonerated. In addition, Jason Farman (2011) argues that users of locative media do not necessary lack presence in physical spaces; on the contrary users embody and simultaneously navigate physical and digital spaces at the same time. To this end locative media co-produce new understandings of presence and space (Farman, 2011) and can channel the space of flows to create a sense of belonging (Berry 2008).
} 
Perhaps this is the point of heterotopias. Not that they exist as a way of categorising, but that as a way of examining social spaces, they give rise to new discourses about what those spaces are, how they arise and what they may mean. New discourses about knowledge, power and society. Which ultimately are reflected in the constitution of our human relationships.

Ransomly provides a new spatial formation, challenging conventions about connectivity. As a disconnected space, Ransom/y reorganises spatial norms, where the user can resist communicative norms like the burden of being constantly available to others via mobile networks. Prager reveals to me that he envisions Ransomly partnering with a company like Starbucks, so patrons could have the option of making "all of their Starbucks [visits] quiet." Ransomly users who visit Starbucks or cafés more broadly to undertake work, have face-toface meetings, or catch up with friends could benefit from Ransomlyby making it harder to respond to a message or check their social media while in the café. Ransom/y calls into question the possibility of connectivity; within the disconnected space the only smartphone functionalities available to users are calls and maps. Once the user leaves the 12-foot radius of the beacon, the Ransomly app shuts itself down with users notified that they are exiting the quiet space.

The concept of heterotopia therefore helps to understand Ransom/y not in binary dis/connected terms, but as the 'other' of connected space. Ransomlyerases the Cartesian distinction of mediated and unmediated space and, by doing so, provides an outside to connectivity by virtue of collapsing the distinction between dis/connection. By erasing the distinction between dis/connection, Ransomly does not extend connectivity culture, but imposes moral limits. 


\subsection{Moral high ground of disconnection}

Ransomly does not merely upend dominant communicative norms, but offers an idea of ab/normal smartphone usage. In other words, a disconnected space such as Ransomly does more than just contest connective culture; it also constitutes the moral high ground in relation to smartphone usage. The association of a disconnected space as a (more) humane space than connected space has the effect of pathologising connective behaviour as unhealthy and positioning offline activities as healthy. In addition, utilising the concept of heterotopia to identify a moral space of resistance appears oxymoronic, even antiFoucauldian, given the concept of heterotopia is most commonly used to analyse spaces that are designed or co-opted for marginalised, abnormal or deviant practices (Beckett, Bagguley, and Campbell 2017; Evans, Riley, and Shankar 2010). A more traditional use of Foucault to analyse Ransomly might draw upon his earlier work on diagnosing mental illness to argue that Ransomly pathologises online behaviour. Jurgenson $(2019,70)$ makes this point in his polemic on disconnection culture, which is worth quoting at length:

Different from critiques of how profit motives structure digital tools often to the end of violating user privacy and autonomy, the wellness framework instead pathologizes any digital connection as inherently contaminating, something one must confess, carefully manage, or purify away. When the 'online' is framed as potentially toxic, users must assume a new responsibility for regulating their exposure. Remembering Michel Foucault's point that diagnosing what is ill is always equally about enforcing what is healthy, we might ask what new flavor of normal is being constructed by designating digital connection as a sickness. Similar to madness, delinquency, sexuality, or any of the other areas whose pathologizing towards normalization Foucault traced, digitality -- what is 'online,' and how one should appropriately engage that distinction -- has become a productive concept for organizing, controlling, and managing new desires and pleasures that have come with the development of communication technologies. 
While Jurgenson is correct to point out that the framing of online behaviour as unhealthy or inhumane is problematic, he neglects to acknowledge that the prevailing culture of connectivity is also by design and ideological, driven by technology platforms (Bollmer 2016; van Dijck 2013) which benefit from the time users spend online and being connected to the internet. His insinuation that disconnection is first and foremost an act of moralisation overlooks that disconnecting can also be an act of moral resistance to the attention economy and culture of connectivity. By utilising positive psychology to create a disconnected space, Prager does not shirk from subjecting users to regimes of power. Instead, Prager resists the attention economy by reorienting a certain strategy of subjectification to enhance the users' wellbeing. In a later interview, Jurgenson admits he "undertakes practical disconnection" by turning his phone into Airplane mode when working (qtd. in Bolin and d'Arnault 2018) and acknowledges that his reservations are about disconnective practices that become ideological. However, Jurgenson does not delve into what the differences between practical and ideological disconnection are. Perhaps trying to articulate any difference between practical and ideological disconnection is beside the point. Ransomly recognises the proconnective environment and that disconnecting from the internet is increasingly becoming impractical. Ransomlytries to make disconnecting more practical by producing a disconnection-by-default space. However, Ransomly is also ideological, telling the user that the disconnected space is a 'space for humanity'. What appears to be a technology of space to resist the attention economy doubles as a technology of power that connects users to new moral regimes about appropriate smartphone behaviour.

Instead of identifying Ransomlyas offering either practical or ideological disconnection, the concept of heterotopia allows Ransomly to be interpreted as a space of moral resistance. Heterotopias alter normative sets of relations, and Ransomlyalters space, 
network protocols and networked reality for inhabitants. The question of ab/normality in the attention economy and digital spaces is highly unstable and political, contested by various actors including internet legislators, advertisers, platforms and morally driven tech entrepreneurs like Prager. We have not inhabited digital space for more than a couple of decades. There is no 'usual' order, no normative consensus concerning the 'right way' to act or move as we have come to expect in physical spaces. And the structure of the internet has radically changed, with the dominant Facebook, Google, Amazon platforms and their large empires of for-profit cloud infrastructures, software systems and applications a far departure from the less commercial internet of the 1990s. These trillion-dollar technology companies have undertaken substantial renovations of our digital quarters. There are a range of people and professions who contest the social order of digital space and seek stability, such as comedians and musicians who admit they find smartphones in the audience to be distracting and risk exploiting a performers' intellectual property (Smith and Keane 2019), anti-capitalist hackers who are concerned about data privacy and the power of platforms (Deseriis 2013), parents and psychologists who have concerns about the long-term effects of screen time (Aiken 2016; Turkle 2013; Twenge 2017), and conservative politicians who share their views (Hawley 2019). Ransomly is just one attempt to contest the social order of digital space.

Ransomly produces a disconnected space that is not a dystopic or utopian vision of space, but a claim to reorder the communicative norms within digital space. In conceptualising disconnected space as a heterotopia instead of a utopia, I have tried to demonstrate that a disconnected space provides a convenient escape from the internet for users. As a heterotopia, manufactured disconnected space is defined primarily by its potential for difference and to resist the dominant communicative norms in connected spaces. A disconnected space like Ransomly offers a different kind of rehabilitative space in 
wider areas of ubiquitous connectivity for inhabitants. I also contend, however, that the alternative ordering of a disconnected space stabilises the social order of digital space and subjects users to new moral regimes of power. In the next chapter, I explore how disconnected spaces can protect users from a regime of power that is even more pervasive. 


\section{A digital sanctuary that provides immunity from surveillance}

In today's era of ubiquitous Wi-Fi and mobile networks, smart devices and Big Data, an increasing number of digital activities are monitored, recorded and analysed. Platforms such as Facebook and Google track which websites users visit, how far they scroll, what they click or hover on, who they interact with, and what they search for. Streaming companies like Netflix and Spotify record when viewers pause or skip content (Vonderau 2017) and rideshare businesses such as Uber or Lyft track users' location data and the times that people access their services to determine the optimal time to offer surge pricing and discounted rates (Cohen et al. 2016). Foucault ([1975] 1977, 200) may have warned that "visibility is a trap" when describing the effects of Bentham's panopticon architecture, but even Foucault could not have envisaged the degree to which surveillance has been integrated into the fabric of digital society.

In this chapter, I examine a hybrid digital and offline magazine called The Disconnect (2018). By forcing its readers offline, I argue The Disconnect is a digital sanctuary that provides a space of resistance to the logic of ubiquitous network surveillance. In the Middle Ages, a sanctuary was a sacred place that provided shelter for inhabitants from illness or violence as well as a temporary legal immunity from corporal or capital punishment (Shoemaker 2011). In the context of disconnecting from the internet, a sanctuary could be a place where users seek refuge from the so-called attention economy, certain surveillance practices, their own technological habits, or more generally, the unrelenting flows of connectivity that are afforded by social media platforms, Wi-Fi and mobile networks. In short, a sanctuary is a space that makes users less visible. 
I first outline why users of the internet might seek out a digital sanctuary. In the first section, I discuss why it has become increasingly difficult to avoid surveillance activities while using digital technologies, as well as the social and individual harms of surveillance. In the second section, I walk through The Disconnect to demonstrate how the web application provides immunity from surveillance. To do this, I turn to critical network theory to extend Foucault's imagined mode of spaces of resistance within the network as opposed to outside

it. Connecting network theorist Mejias' concepts of nodocentrism and paranodality to Foucauldian resistance, I argue that The Disconnect disrupts the nodocentric or dominant network logic of connectivity that undergirds the threat of totalising surveillance by shifting users offline while they remain using digital technologies. By shifting users offline, I argue The Disconnect provides a minimal space of self-integrity for users for the purposes of reading. I close with a discussion of the privilege of immunity from surveillance.

\subsection{The erosion of a space for the self}

What is the significance of The Disconnect, a digital technology that can operate both inside and outside of the network? How can such a technology provide a digital sanctuary and inoculate users from surveillance practices? The significance of a digital sanctuary, I argue, is that it is increasingly difficult to avoid surveillance while navigating digital space. In this section, I discuss the normalisation of surveillance activities in digital spaces and, in particular, dataveillance, which is the practice of monitoring and collecting online data (Raley 2013). I begin with a discussion of the everyday extraction of human experience.

\subsubsection{The extraction of human experience}

An increasing number of online spaces are subjected to surveillance. One of the major reasons why digital space has become fused with surveillance logics is because of the 
emergence of a new type of capitalism that underlies many tech platforms that govern digital space. In her book The Age of Surveillance Capitalism, Shoshana Zuboff (2019) comprehensively traces the emergence of this type of capitalism and its impacts on individuals and the fabric of society. Zuboff $(2019$, i) defines surveillance capitalism as "a new economic order that claims human experience as free raw material for hidden commercial practices of extraction, prediction, and sales." Throughout her analysis, Zuboff deploys metaphors to emphasise the widespread significance and harms of surveillance capitalism and compare its means of production to prior energy or industrial industries. The chief metaphor that Zuboff uses is describing the technology industry as extractors. According to Zuboff (2019), the technology industry extracts data from users' activities to generate insights into user behaviours and consumerist preferences, in order to maximise their advertising revenue. Zuboff contends that surveillance capitalism subjects humans to unprecedented behavioural manipulation and has a more invasive means of production compared to previous forms of capitalism. Whereas industrial capitalism depended on people as consumers and employees, surveillance capitalism anticipates the behaviour of individuals and populations and actively seeks to undermine individual self-determination and autonomy for the purposes of an unhindered flow of behavioural data (Zuboff 2019).

Zuboff attributes the foundations of surveillance capitalism to inventions by Google and Facebook. Google's development of Page Rank, AdWords and AdSense revolutionised extraction by providing a means to measure the perceived importance of website pages and an online advertising market (Vaidhyanathan 2012). Click-through rates (CTR) was the first pricing metric for the online advertising market, measuring the relevance of an advertisement to corresponding users. CTR appeared to provide a much more accurate means to measure the effectiveness of advertising than audience measurement techniques in broadcasting or 
print, which has had the effect of attracting advertisers to Facebook and Google and disrupting the news industry (Wu 2016). Facebook's major contribution to surveillance capitalism was the Social Graph, which provided the largest social network dataset about users' relationships, activities and interests (Ugander et al. 2011). Taken together, these innovations create an imperative for surveillance capitalists to extract as much data from users as possible. User surveillance therefore morphed into a logic of accumulation that has created asymmetries of power, extending surveillance activities to the workplace, activities of online consumers and competitors in the market and of proprietary assets (Fuchs 2012). The behavioural surplus that tech platforms generated is a zero-cost asset that diverted user data from service improvement to highly lucrative market exchange. In all, Google and Facebook have "institutionalised behavioural surplus as the cornerstone of a new kind of commerce that depended on online surveillance at scale" (Zuboff 2019, 83).

Surveillance capitalism and the extraction imperative have significantly changed the way content is published in the attention economy. Surveillance capitalism-related business models such as web analytics have made the attention of audiences and users even more valuable to publishers. Web analytics are commonly used by the online publication industry as they provide rich insights into reader preferences-what headlines they click on, sentences they highlight, or how far they scroll-which in turn are used by publishers to inform editorial decisions on subsequent content (Lee, Lewis, and Powers 2014). Web analytics are even used by editors to dynamically rewrite headlines if the CTRs are underperforming (Kuiken et al. 2017). Edson C Tandoc, Jr (2014) considers web analytics to be instrumental in the media industry adoption of a more consumer-driven process of selecting and packaging content in order to increase web traffic. 
Web analytics and the associated extraction imperative have also changed the way content is delivered to readers. The truncated article provides a recent example, where readers have to click a "read full story" button to reveal the entire article, while those who do not can keep scrolling and are presented with alternate articles, navigation, or even advertisements. The truncated article functionality creates data on the number of users who read the content beyond the headline and provides more certainty that the user will see an advertisement (Harley 2015).

Fewer digital spaces are exempt from the extractive imperatives of surveillance capitalism. As Zuboff $(2019,99)$ provocatively puts it: "Human experience was Google's virgin wood," with an increasing number of online spaces fitted with web analytics to collect and analyse users' activities. The extraction of human experience by surveillance capitalists draws similarities to the self-inspection techniques that Foucault ([1976] 1990) argued were instrumentalised into the early medieval Christian confession. The technology of selfexamination was turned by the Catholic Church into an honest confession to a priest. Such techniques of confession were integral to the functioning of pastoral power, or the formalisation of the intrusion into the private lives of individuals to learn about their secret inner existence and provide spiritual direction (Foucault [1976] 1990). The exteriorisation of interiority in the $21^{\text {st }}$ century is much more widespread, according to Michael Harris (2017, 228-229), arguing that tech platforms chip away at users' solitude and quiet spaces:

What we're beginning to notice, from the midst of this tech-gorged moment, is a resource we can either nurture or allow to be depleted. Think of a forest. For centuries we could walk among dense stands of firs when we chose, or profit from cutting the same trees down without much care as to whether nature at large would be materially damaged. Then a line was crossed and we found ourselves starved for green space. Today, thanks to platform technologies, profit is wrought as much by the dismantling of mental resources as it is by 
the dismantling of natural resources. We have learned to harvest the solitude of others.

Profiteers produce social grooming technologies, and agents of distraction swarm around us. Solitude is consumed and depleted as surely as Brazilian rainforests are toppled.

Surveillance capitalism also provides Google and Facebook with what Zuboff $(2019,352)$

calls instrumentarian power, defined as "the instrumentation or instrumentalization of behaviour for the purposes of modification, prediction, monetisation, and control." Subjected to instrumentarian power, users are no longer ends in themselves, but become the means to others' ends. Users become unwilling suppliers of raw material to analytics platforms as larger cycles of revenue generation. Legal scholar Mark Bartholomew $(2017,2)$ similarly describes the dangers of ubiquitous advertising as pernicious: "when no space is off-limits to commercial appeals, we become numb to the ideology of advertising, lowering our defences, accepting Madison Avenue's suggestions for self-definition, and no longer considering, nonmarket-based perspectives." What Bartholomew implies is that the effects of instrumentarian power on people is subtle, where market-based values such as consumption and choice slowly crowd out other norms, like civic duty, a sense of community or charity to others.

\subsubsection{A space for the self}

The Age of Surveillance Capitalism (Zuboff 2019) received notable criticism for focusing on surveillance at the expense of capitalism (Morozov 2019) and neglecting to cite existing scholarship that has problematised the development and spread of surveillance (Haggart 2019). By not sufficiently grounding her work in humanities and social sciences, Zuboff misses the opportunity to build upon other scholarly insights about the impact of surveillance capitalism on digital and other shared spaces. To highlight a few important insights, Andrejevic (2007) argues that the surveillance capabilities embedded in platforms have enabled individuals and businesses to privatise the data resulting from users' immaterial 
experiences that take place on those platforms. Critical data and legal scholars draw upon Foucault to note the 'soft-biopolitics'1 of algorithms and data analytics that govern users by recognising and anticipating users' desires (Cheney-Lippold 2011, 2017; Rouvroy 2013). There is also critical political economy, architecture and urban studies scholars who comprehensively demonstrate that the 'smart city', or public spaces embedded with smart devices and data touch points, atomise collective experiences and prioritise the interests of capital and neoliberal government over wider civic values (Cardullo, Di Feliciantonio, and Kitchin 2019; Green 2019; Greenfield 2017; Mosco 2019).

Zuboff also neglects to articulate any means of resistance to surveillance capitalism. In a similar problematisation of ubiquitous surveillance, Couldry and Mejias (2019) articulate the need for a space for the self to resist being visible. The authors argue that a sense of space is important for individuals to develop relative independence from power. Subjecting digital spaces to surveillance and advertising erodes the ability of digital space to communicate non-commercial values. Many forms of advertising or sponsored content are hard to detect (Bartholomew 2017), with advertising acting as a technology of power that normalises disciplinary power (Foucault [1975] 1977) by shaping user's sense of self while masking its ability to do so. Couldry and Mejias (2019) argue that surveillance practices invade the space of the self by making submission to tracking a requirement of daily life. Similar to Zuboff, they argue that a fundamental law of dispossession is imposed on digital spaces that "continuously invaded and subjected to extraction by external power" (Couldry and Mejias 2019, 157).

\footnotetext{
${ }^{1}$ See section 4.2 for a discussion of the similarities between biopolitics and surveillance capitalism.
} 
Drawing upon Hegel ([1807] 1977), Couldry and Mejias argue that individuals need a metaphysical sense of space or a minimal space of self-integrity. They contend that Hegel thought that, for anyone to be free, they must have access to an 'external sphere of freedom' that defines them: "By installing automated surveillance into the space of the self, we risk losing the very thing-the open-ended space in which we continuously monitor and transform ourselves over time-that constitutes us as selves at all" (Couldry and Mejias 2019, 161). A space of minimal self-integrity would guarantee what James Williams (2018) calls a "freedom of attention", where attention is understood as the operation of will instead of a clinical interpretation. Williams (2018) describes having a freedom of attention as more than just being disciplined or productive but having the capacity to care in the first place, to generate values and goals, and act upon them.

\subsection{Paranodal disconnection via The Disconnect}

To conceive of a space to resist surveillance capitalism, I first turn to Foucault's concept of biopolitics. There are some similarities between surveillance capitalism and what Foucault called biopolitics, or the shift by Western governments in the early $20^{\text {th }}$ century to use data to manage the lives of populations. Foucault $([1976] 1990,138)$ saw biopolitics as a political logic that administered life and populations as its subject: "to ensure, sustain, and multiply life, to put this life in order." Foucault (2008) used the concept of biopolitics to describe the development of political power in the early $20^{\text {th }}$ century when Western states shifted from taking away lives (what he called sovereign power) to a more modern power of administering the lives of its citizens. The emergence of medical and psychiatric sciences and modern educational institutions regulated populations as statistical phenomena, measuring collective health and reproduction (Foucault [1976] 1990). Surveillance capitalism 
is similarly biopolitical to the extent that capitalism's new goal is the commodification and operationalisation of life or human experience for the purposes of governance. Yet the term biopolitics attributes agency to the goals of the state. In the context of surveillance capitalism, the governance of human experience or life is at stake; however what drives surveillance capitalism is not politics or the goals of government, but instead the maximisation of profit (Couldry and Mejias 2019; Zuboff 2019). Yet Foucault's biopolitics is useful as a starting point for the possibility of a space of resistance. Despite life becoming the new aim or object of power, Foucault ([1976] 1990, 143) argued that life cannot become fully captured by governance measures because "[life] constantly escapes them". Foucault saw the possibility of resisting the capture and operationalisation of human experience, but he was less interested in conveying how a space of resistance could be achieved.

Mejias (2013) offers the theoretical concepts of nodocentrism and paranodality that I believe allows for an articulation of Foucault's space of resistance. Instead of refusing digital technologies, Mejias calls for a rejection of the logic that digital technologies typically impose: nodocentrism. Mejias $(2013,10)$ defines nodocentrism as a dominant network logic that "rationalises a model of progress and development in which those elements that are outside the network can only acquire currency by becoming part of the network." Nodocentrism reduces digital technologies to mere nodes that serve to connect to other nodes, thereby imposing a connective imperative upon digital technologies and networks. Yet, as I have argued in this thesis, users engage in disconnective practices while using digital technologies, such as deleting a post on Facebook or blocking another user profile on Twitter (Light 2014). Moreover, Grant Bollmer $(2016$, 2-3) disputes that networked connection is a naturally sought after state, arguing it was not until the later development of financial and business technologies in the mid-20th century that "connection [was viewed as solving] ...social 
problems, reflecting a desire for connectivity found in the primal yearnings of the human soul". To challenge nodocentrism, Mejias $(2013,153)$ recommends the paranode as a conceptual starting point, a term that derives from neuroscience and refers to a "specific type of cellular structure that, while not part of the neural network, still plays an important role in excitatory signal transduction." An example of a paranode is any technology, object, or person that sits outside the network and resists assimilation to network logics, such as obsolete hardware or software, internet jammers, or a cut-off web link (Mejias 2013).

I approach The Disconnectas a paranodal technology that operates both within and outside the digital network. By forcing its readers to disconnect their device from the internet, The Disconnect temporarily occupies a paranodal space on the edge of the network that is not subject to dataveillance. Additionally, The Disconnectrejects nodocentrism by demonstrating how digital media technologies can disconnect to create valuable user experiences that are optimal for independent activities such as reading. To analyse The Disconnect as a paranodal web application, I turn to the walkthrough method (Light, Burgess, and Duguay 2016) and offer a user perspective of the first issue of The Disconnect. However, the scope of the walkthrough method is too limited to capture how The Disconnect leverages the interactive capabilities of the web to transition to paranodal status. I therefore supplement the walkthrough method with a platform studies approach to analyse how The Disconnect interacts with application processing interfaces (APIs) to protect users from data collecting practices. These approaches are informed by an interview undertaken with The Disconnectco-founder Chris Bolin. Combining both the walkthrough method and a platform studies approach enables both a description and analysis of the unique user experience and interactivities of The Disconnect that, in turn, imagines new opportunities and applications of digital technologies beyond surveillance. 


\subsection{Navigating The Disconnect}

The predominant creative force behind The Disconnect is Chris Bolin, a software engineer with a background in developing web applications that interact with the web. Prior to The Disconnect, Bolin (2018) built a one-page website called Offline Only that forced users to go offline in order to read a short essay Bolin had written. In the essay, Bolin writes about the distracting nature of the web, how connectivity can hinder focus and productivity, and the benefits of disconnection. Offline Only received popular acclaim from a number of mainstream media outlets. ${ }^{2}$ The positive feedback of Offline Only convinced Bolin to try an even more ambitious offline web application: a hybrid online/offline magazine. To do so, Bolin enlisted the help of editor Clayton D'Arnault and invited writers to submit fictional stories, essays, and poems to partake in a media experiment. Writers were informed of the limitations of a hybrid offline/digital magazine and instructed not to submit any work with hyperlinks or other components that require a connection to the internet to function. The project was titled The Disconnect and the first issue launched in February 2018.

To access The Disconnect, readers must first visit the magazine's website at http://thedisconnect.co (Figure 4-A).

\footnotetext{
2 The launch of Offline Only was covered by international media publications such as Vice, Motherboard and The Next Web, as well as the Canadian Broadcasting Company.
} 


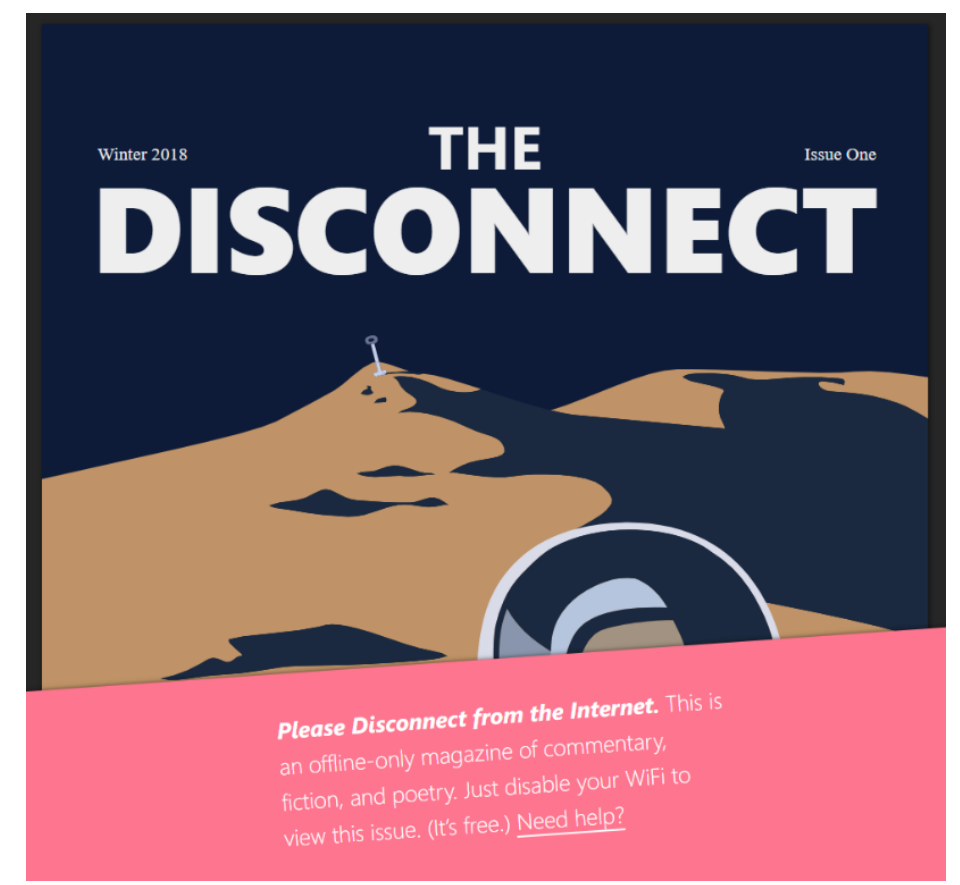

FIGURE 4-A: THE DISCONNECT MAGAZINE HOMEPAGE DESIGNED BY JACOB HALTON

The Disconnect homepage resembles the front page of a magazine with a cover image, magazine title, issue date and number. The homepage offers limited interactivity; the screen is locked, and the user cannot scroll in any direction. The only clickable element is a hyperlink reading 'Need help?'. ${ }^{3}$ The instruction asking users to disconnect from the internet appears as a digital bellyband, which are typically used in the print industry to highlight featured content and package magazines to prevent readers from browsing the magazine before purchase, as a print magazine cannot be opened without breaking the bellyband (McKay 2019). The bellyband on The Disconnect forces a different type of commitment from potential readers. There is no paywall or financial transaction required to unlock any content. According to Chris Bolin, the bellyband on The Disconnect serves as a "pay-attention wall" (qtd. in Ingram 2018) by forcing users to disconnect their device from the internet to eliminate any third party digital distractions. Once the user has disabled their connection to

\footnotetext{
${ }^{3}$ The text on the publisher's band slightly varies depending on what device the user is using to access The Disconnect.
} 
the internet, the homepage of The Disconnect reloads, transitioning to a black and white version of the same front cover image sans the publisher band (Figure 4-B). If the user reestablishes their connection to the internet, the bellyband reappears.

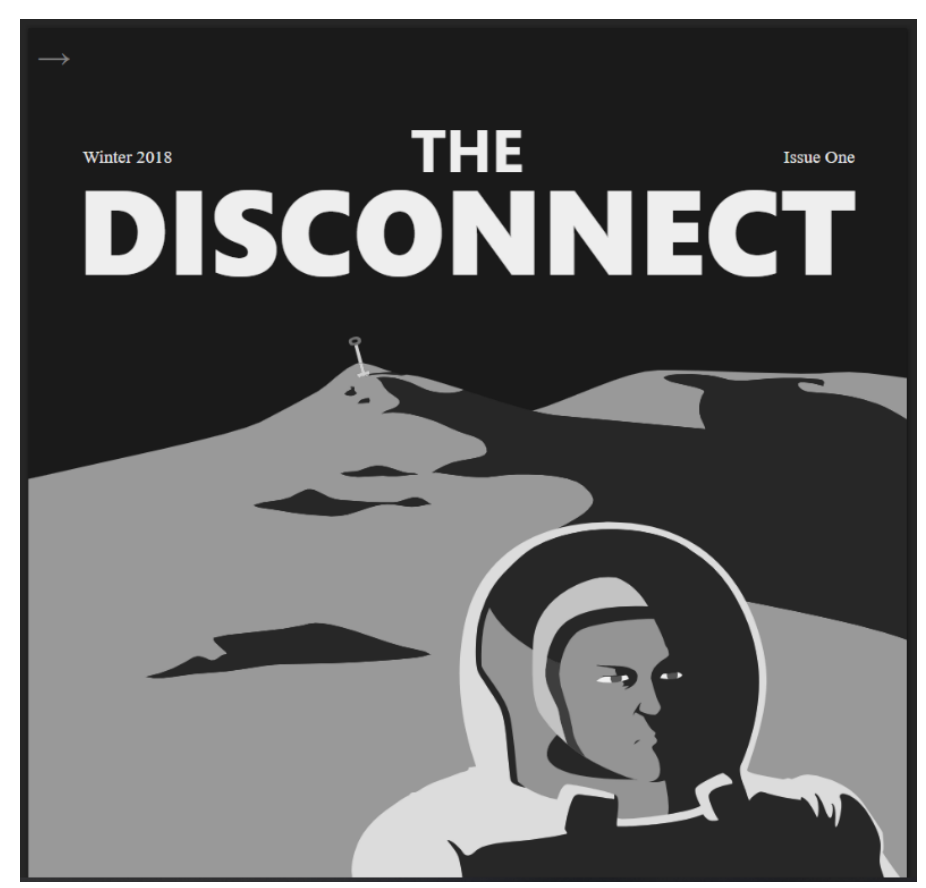

FIGURE 4-B: THE DISCONNECT MAGAZINE AFTER THE USER DISCONNECTS FROM THE INTERNET

After disconnecting, The Disconnect enables the user to scroll downwards, which reveals the contents of the magazine (Figure 4-C). Articles are categorised under the headings of 'stories', 'commentary', 'poetry', or 'about' for supplementary information about The Disconnect. Each article is listed as a line item and displays basic metadata, including the title, author, and length of the article in page. If the user hovers their cursor over a line item, the text on the line item moves subtly and the font colour changes to a lighter shade of grey. Clicking on the text takes the reader to the article which is visually distinct from the section of The Disconnect that houses the contents. 


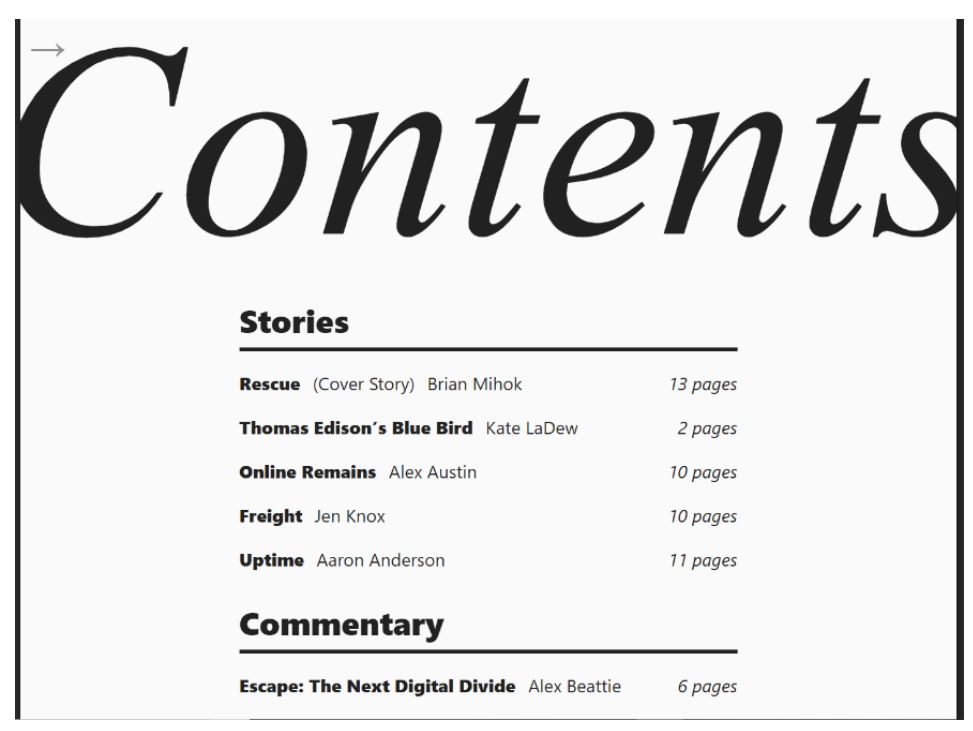

FIGURE 4-C: THE CONTENTS OF THE DISCONNECT MAGAZINE

The first issue of The Disconnect contains thirteen articles, with each article presented to the user as a distinct web page. ${ }^{4}$ Users can browse between each article and return to the homepage or click on next article at the footer of each page. However, The Disconnect offers no interactivity outside of itself. Each article is effectively siloed from the rest of the web, devoid of hyperlinks that can transport users to places on the network beyond The Disconnect. If the user attempts to open a tab on their browser and access another website, they are promptly reminded by their browser that they are offline and/or to check their connection. As Figure 4-D demonstrates, when reading the content of The Disconnect, there are no auto-playing videos or banner ads to click on; users can only read.

\footnotetext{
${ }^{4}$ Each article on The Disconnect has a unique URL slug.
} 
I $\mathrm{n}$ the morning Tom contemplated leaving the crash site. He looked over at the mound where he had buried Colonel Ames, the handle of the shovel sticking up as a makeshift marker. It appeared he was in some kind of desert, so there was little choice but to seek shelter. It was early and still dark. He looked up at the sky to see short bursts of light, as if meteors had lost their will to streak across the atmosphere and instead burnt out immediately.

Tom couldn't bring himself to turn off the signal in his helmet. When he made a sound, breathing or grunting or speaking quietly to himself, he heard it through the receiver as if he were on the phone. He didn't want to risk removing the helmet either. Microbes, fungus, and insects were all good cause to keep himself protected until his exposure to the environment was forced. His oxygen would last up to eight hours set at the lowest flow rate, though he wouldn't survive long like that. He could also refill with the capsule's reserve, after which he would have no choice but to breathe the planet's air.

FIGURE 4-D: ANARTICLE ON THE DISCONNECT MAGAZINE

The articles and layout in The Disconnect also lack "rich content", meaning high quality images or videos. In this sense, The Disconnect is aesthetically minimalist, offering only simple black and white typography and an abundance of white space. However, what The Disconnect lacks in vibrant colour or imagery, it makes up for by offering a unique online reading experience, ideal for fiction or longer forms of writing. Without hyperlinks or banner advertisements, The Disconnect is designed so readers cannot be distracted easily. There are no opportunities to share, "like" or otherwise circulate the content of The Disconnect. Readers are shielded from any such nodocentric logic that prioritises the circulation of content over the actual engagement with content. Bolin considers that a distraction-free online space offers a novel web-based reading experience:

I think The Disconnect may have opened up something unique on the web, for example, the cover piece is a story called Rescue, it's a pretty long piece and the author had said that he had never find a place for it online because all of the restrictions were something like four 
thousand words or something like that...I was really happy because we could open up something like that. I know multiple people read the whole thing all the way through, and they didn't expect that to happen online. They didn't expect to read a webpage with that much text...So I'm really happy that we're able to do that. We are a very easy fit for fiction and poetry.

Studies comparing offline to screen-based reading have concluded that screen-based reading promotes skimming, keyword spotting, and other forms of fragmented reading which in turn contributes to losses in sustained attention (Liu 2005). The Disconnect complicates such a characterization of screen-based reading by forcing its readers to transform their screen into an offline device. In this sense, The Disconnect is comparable to early e-readers in the mid2000s, such as the Sony Librie or the Amazon Kindle that offered only limited network functionality as they predominantly dedicated to the single function of reading long-form text (Griffey 2012). By removing the distractions that a connection to the internet entails, The Disconnect attempts to fuse an offline reading experience with a digital medium to create an ideal screen-based space where attention can be sustained and oriented for possible pedagogical purposes. The Disconnect therefore reimagines digital environments that could be optimal for reading or any other independent modes of learning or creativity.

\subsubsection{Lifting the curtain: the interactive Web 2.0 and offline $A P I s$}

To a user, an offline digital reading experience may appear impossible. In reality, Bolin utilises the interactivity of the web as a sleight-of-hand. The first trick Bolin uses is frontloading the entire magazine to the user's internet browser. Unbeknownst to users, as they enter the URL of The Disconnect and download the homepage, the entire web application is saved to the browser's web cache. This process is different to most modern websites that load on an as-clicked and as-scrolled basis due to the heavy data transfer 
requirements that websites demand when using rich content. While the average website depends on a steady connection to ensure full functionality, The Disconnect must be entirely front-loaded in the user's internet browser because of the subsequent requirements of the user to disconnect from the internet. As Bolin explains:

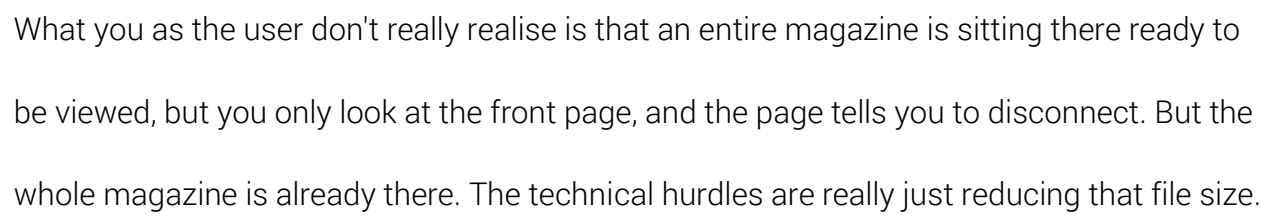

Because it must be front-loaded and read offline, The Disconnect must maintain a small file size; it is a media object that is barely over 200 kilobytes, which is tinier than the poster image on most websites. ${ }^{5}$ The second engineering trick Bolin uses is reversing the intended use of a standard software protocol that has been common to Web 2.0. Web "1.0" and "2.0" are software engineering terms to describe how the web has evolved over the last two decades. The Web 1.0 refers to a network of channels to distribute and publish static Hyper Text Markup Language (HTML) pages of information while, in contrast, Web 2.0 is a platform that interacts with third party web applications or other software programs (Helmond 2015). Bolin is an expert in building Web 2.0 applications: "my day job is to program for the web and that's what I feel like a lot of my skills and expertise is being built up in; thinking about what browsers can do." Web 2.0 applications like The Disconnect are primarily developed through standard software protocols, otherwise known as APIs. The API is a software interface that offers standard access to data, functionality, and protocols. An example is the use of the Facebook API which allows users to sign up for Spotify using their Facebook account. The

\footnotetext{
${ }^{5}$ This in part explains the simple aesthetics of The Disconnect. If The Disconnect contained richer aesthetics: colour, video or any objects high in bytes, The Disconnect would take more time to download.
} 
use of an API in this way is nodocentric because it ensures a greater flow of data throughout the network by connecting more nodes via the interoperability of APIs.

However, just because APIs are predominantly designed to share data does not mean APIs must be used in such a nodocentric way. When users of The Disconnect disable their Wi-Fi, an event is fired by an API that is native to most modern internet browsers. The API is called online and offline events ("offline API"), which was designed to recognise unreliable internet connections and cache content to avoid data loss (Mozilla n.d.). The offline API is typically used by applications such as Gmailto inform the user when they are not connected to the internet and to preserve the user's data. In this instance, Bolin utilises the offline API to instruct The Disconnect web application to remove the bellyband and unlock the content when the user disables their internet connection. If the user is using an internet browser that doesn't have the offline API installed then an alternative method of polling is used to test is the user has gone offline. According to Bolin this happens in a matter of microseconds. To analogise, if the offline API is a lock, then the act of user's disconnecting their device from the internet is the key. Bolin was inspired to flip the logic of the offline API by a much older technology:

I take a little bit of inspiration from the very boring but very powerful changes in washing machine technology. You have a top load washing machine, and this was the technology we had for probably almost a century. And then someone had a very but very profound idea: "why don't we rotate [the washing machine] ninety degrees?"...I kind of think of using offline APIs to do something like this as similar. You're taking the thing and you're basically using it in reverse right? If before it was supposed to notify you when you were in a suboptimal position because you were offline, now we're saying you're in a suboptimal position because you're online. 
By reverse engineering the offline API, Bolin and The Disconnect demonstrates the web can interact with digital technologies without being nodocentric. That is to say, the web does not have to necessitate the flow of data on the network but, at a more fundamental level, can interact with other nodes such as web applications for purposes that are beyond connecting. To analogise, the web is not just a pipe to circulate the movement of data but can also be leveraged as a valve to condition or impede data flow when or where desired. The Disconnect is a 'valve' type of web application as it hinders the flow of data during the point of media consumption and is therefore an example of what Mejias (2013) considers an intensification of the network: an act of creative disassembly that pushes the network to the limits of interactivity beyond nodocentrism. Instead of abandoning the network, The Disconnect reimagines how the network, the web, and APIs can interact to create a novel offline screenbased experience. As Helmond $(2015,3)$ states, APIs enact the "programmability of the web" which does not always transmit to the connectivity of the web, as not all of the web is social (cf. van Dijck and Poell 2013).

\subsection{Immunity from surveillance}

The Disconnect is not just an experiment in the web, but also in the limits of surveillance. The only surveillance that The Disconnect enables is the server-based surveillance required to identify the web location of the reader so a copy of The Disconnect can be sent to the reader's internet browser. The level of information that server-based surveillance offers is rudimentary web location-based data and analogous to what a mailing list offers to a print publication. Beyond the location of the reader's internet browser, Bolin admits he knows next to nothing about the readers of The Disconnect. 
...we don't know the most basic thing of all - which is did [the user] even disconnect? Think of one of those free magazines that just has a bunch of ads-people pick them up from the newsstand and they might just look at the cover and just toss it. That could have been a request for The Disconnect.

The other form of surveillance commonly undertaken on the web is client-based surveillance or web analytics that I discussed in the first section of this chapter as a specific application of surveillance capitalism. Web analytics require a steady connection so a third party data analytics client, such as Google Analytics, can actively monitor the activities of the user. Web analytics cannot be undertaken on The Disconnectas, during the period of media consumption, The Disconnect is paranodal and off the network. The Disconnect can be interpreted as a response to Alexander Galloway and Eugene Thacker's (2007) call for "tactics of non-existence"; the content of The Disconnect can only be consumed while users are offline and, therefore, does not exist to be analysed as nodes of data on the network. The Disconnect is therefore fatal to web analytics practices, offering readers immunity from any data analytics that require a connection to the internet to work. According to Bolin, it would be possible to embed web analytics software within the code of The Disconnect, which could allow data analytics to be undertaken when the user is offline. However, for the data to be returned to Bolin or a third party, the user would have to reconnect to the internet while they still have the web application open. In other words, a forced disconnection significantly reduces the possibility of the successful transfer of captured data analytics. Put slightly differently, paranodality offers a type of immunity that is analogous to a witness protection scheme. By forcing readers to disconnect from the internet, The Disconnect temporarily relocates the reader off the network, hiding them from the gaze of any watcher. 
The Disconnect also provides a space of minimal self-integrity. Readers of The Disconnect obtain a particular type of privacy called 'intellectual privacy'. Neil Richards (2008, 387) defines intellectual privacy as "the protection from surveillance or interference when we are engaged in the processes of generating ideas." In networked environments, intellectual privacy is hindered when the reader is encouraged to respond in a certain way. An example is the blogging platform Medium or later versions of the e-reader Kindle, where web analytics have enabled the development of the 'top highlights' functionality that discloses to readers how many other users have highlighted a certain quote. The top highlights functionality suggests to the reader a collective consciousness of what material other readers consider to be significant, which could encourage the reader to think the same. To this end, inoculating readers from web analytics enhances the intellectual privacy and autonomy of the reader as the absence of social functionalities allows the reader to respond to a text on their own terms.

\subsubsection{The privilege of a digital sanctuary}

Resisting the attention economy via lifestyle adaptions is a privilege. Many adaptions require using products and services that cost money, are not universally available, or require degrees of media literacy or technical knowledge to use. When the manufacture of disconnection positions the disconnection as between the user and the attention economy, the user is no longer paying with their attention and therefore have to pay another way. Paying for access to content with one's attention and data is why privacy has become a luxury-not because users do not care about their privacy, but because free access to platforms is given in exchange for trading their privacy (Papacharissi 2010). It also takes privilege to be even concerned about loss of attention or have the agency to resist surveillance, considering it is increasingly common for surveillance capitalists like Google and 
Facebook to provide subsidised infrastructure, hardware and software products and services for initiatives on the proviso they can harvest the data that is generated from them (Kuehn and Daubs 2016). Until a seismic shift of funding models occurs in the media and technology industry, resisting the attention economy will continue to come at some sort of cost and only be available to the affluent (Beattie 2018a). While the commodification of disconnection is welcomed in tourism and management studies as an opportunity to tap into an emerging market (Paris et al. 2015; Tanti and Buhalis 2017), the issue remains largely unexplored in media studies.

The Disconnect is not only disconnected from economic imperatives of the attention economy, but largely from economic activity in general. Bolin built The Disconnect in his spare time via the support of his full-time job as a developer for a software company. All three editions of The Disconnectare free to download for readers, bucking a business trend in the attention economy where free content can only be free to the reader because it is typically monetised via web analytics and the placement of advertisements throughout the content. By choosing to keep The Disconnect both free for consumers and of advertising meant that Bolin could not initially pay its writers. Contributing writers were asked to contribute for free for the first edition, meaning only privileged voices who did not require the income from providing a writing contribution were in a position to be involved. We can therefore conclude that The Disconnect is not a sustainable media business model but challenges the attention economy in ideas only.

Where The Disconnect challenges the attention economy in ideas, is by presenting an alternative trade of user attention to access content. For the second edition, Bolin was approached by the organisation Mozilla to sponsor the issue, which allowed Bolin to pay his writers. Mozilla (2020) is a non-for-profit organisation that makes free software (such as the 
internet browser Firefox) with a mission statement to "keep the internet open and accessible". Bolin told me that he was comfortable accepting sponsorship money from Mozilla because he agrees "in the specific instances of what [Mozilla is] doing". Sponsored content, or native advertising has become a ubiquitous business model for mainstream media (Ferrer Conill 2016) and is when advertising is embedded in content in the guise of news with the goal to undermine a news readers ability to discern between independent news stories and content that is paid for by commercial customers (Amazeen 2019; Wojdynski 2016). Bolin is adamant that sponsors should remain relatively disconnected from the purview of readers. To achieve this separation, he ensures readers of the second edition of The Disconnect can clearly discern between independent content and content paid by Mozilla. Mozilla's advertisement is contained to a separate page from the rest of the magazine. Bolin tells me that it was important to him to not allow the Mozilla advertisement to fragment the attention of readers while they engage with the content of the magazine: "[The advertisement] wasn't going to be in the middle of other pieces, it could be set aside in its own place, and I think that's kind of unique." By clearly separating commercial material from the editorial content of the magazine, The Disconnectappears to resist 'brand journalism', an increasing prevalent type of media production that blends advertising and news genres together (Serazio 2020).

The Disconnect is an experiment in software and media that suggests there is more to digital technologies than fragmenting the attention of users and subjecting them to surveillance. Surveillance and web analytics depend upon the nodocentric belief that digital technologies predominantly serve to connect to other nodes on the digital network and facilitate and generate data flow. The Disconnect rejects the connective imperative and was built on an opposing paranodal view: that disconnection from the network and the attention economy can be a desirable or optimal state and become normalised. By reverse engineering 
an offline API, The Disconnect forces its readers to disconnect beyond the reach of web analytics. The Disconnect therefore simultaneously celebrates certain features of networks and digital technologies such as ease of access and dissemination, while eliminating the ubiquity of surveillance that has become a cost of admission to the internet. Furthermore, by removing distractions and protecting intellectual privacy, The Disconnect further imagines new possibilities of screen and/or network-based reading environments that could be optimal to certain modes of independent learning. Paranodality and the idea of disconnected digital experiences offers a means to break the entrenched connective imperative of networks and the overwhelming power that totalising surveillance has over our lives. 


\section{Part III}

The production of the disconnected subject 


\section{The fixable self and the ethical turn of persuasive technology}

"[They] call me both someone who runs a game reserve and is a poacher," says Tim. ${ }^{1}$ । am sitting in a café in Venice Beach, Los Angeles, speaking to Tim, who is a behavioural scientist and software developer (what I refer to as a "persuasive technologist") and senior executive at behavioural software company Boundless Mind. Tim is responding to a question I put to him about a possible conflict between Boundless Mind's popular behavioural software products. One of the products is an API for third party businesses to optimise the capture of end user attention. The API accesses behavioural data from third party commercial software (e.g. a medication adherence application) and applies machine learning and behavioural design techniques to enhance the persuasiveness of interactive features. Another is an application called Space which allows users to make third party applications less persuasive and 'fix' the habit of mindless smartphone scrolling. In effect, these products suggest that Boundless Mind both "poaches" and "protects"2 end user attention, which has attracted notable mainstream media attention (Parkin 2019; Shieber 2017).

However, Tim does not believe that Boundless Mind's products are in conflict with each other. From a legal perspective, he has a point; the responsibilities of persuasive technologists like Boundless Mind have yet to be formalised into professional codes of conduct $^{3}$ and largely exist as guidelines in design books (Eyal 2014; Fogg 2001), blog posts

\footnotetext{
${ }^{1}$ Tim is a pseudonym. The employee I interviewed from Boundless Mind requested anonymity.

${ }^{2}$ My intention is not to suggest that persuasive technologies neatly fall into the either "poach" or "protect" categories in relation to user attention. What makes Boundless Mind unique is that the company optimises the capture of end user attention and resistance to said techniques. I use "poach" and "protector" terms because my interviewee identified with them.

${ }^{3}$ There is however, work underway to establish ethical design standards and a professional code of ethics for designers and persuasive technologists. For examples, see User Experience Professionals Association (Shamonsky 2018) or the Center for Humane Technology (2019).
} 
(Harris 2016), or arguments by philosophers and legal scholars (Dorrestijn and Verbeek 2013; Frischmann and Selinger 2018; Verbeek 2009). Yet Tim does not even believe that his company is acting unethically or in bad faith, instead justifying their software products as a response to the variety of end user interest in behavioural change and evidence that Boundless Mind is "defending [the] cognitive liberty [of end users]." On the surface, Tim's claim that his company defends cognitive liberty appears to be the kind of empty selfposturing that is typical of a spokesperson. However, as I explore in this chapter, Tim's comment reflects a perception of Boundless Mind, and persuasive technologists more broadly, that their technological inventions can 'fix' the undesirable tech habits of users and are enablers of an ethical or good life. Boundless Mind's credentials were affirmed in 2019 when the company was acquired by corporate and consumer wellbeing conglomerate Thrive Global. Thrive Global has raised over 50 million USD in funding (Crunchbase n.d.) and has the goal of being a leader of behaviour change. In a statement about acquiring Boundless Mind, Thrive Global founder Ariana Huffington (2019) stated: "We'll never be able to change behaviour at scale if we don't use all of the tools in our toolkit, including the most cuttingedge technology."

In this chapter, I critically assess how Boundless Mind 'fixes' the smartphone unconscious scroller. In the first section I outline the expertise of persuasive technologists. I trace the development of persuasive technology by key figures such as B.J. Fogg and Nir Eyal, as well as Boundless Mind. By identifying subjectivities in relation to user behaviour and encoding such knowledge into models and software, I argue Fogg, Eyal and Boundless Mind are experts in "mobilising" (Miller and Rose 2008) the end user without overtly manipulating them. In the second section, I undertake a walkthrough of the app Space to demonstrate how 
Boundless Mind put their expertise about user subjectivities to therapeutic ends. In particular, I highlight a behavioural technique embedded in Space that enables the user to develop a transformative relationship with their technology habits and persuasive applications. In a society where individuals are encouraged to pursue wellbeing for personal fulfilment, the expertise of Boundless Mind places them in an ideal position to offer the therapeutic cure to persuasion. I conclude the chapter by arguing that a mode of resistance based on the user fixing their habits obfuscates the power of persuasive technologists by limiting the scope of ethical enquiry to the activities of the user.

\subsection{Mobilising the end user}

Persuasive technologies use behavioural science to non-coercively shape the actions of end users to a predetermined goal (Verbeek 2009) and have become common place in digital media technologies and formalised in various disciplines. Perhaps the most established use of persuasive technologies are 'nudges' or choice architecture (Thaler and Sunstein 2009), which is a subfield of behavioural economics that debates the use of persuasion to enhance public policy outcomes in Western democracies. A famous example of a nudge is an automated teller machine (ATM) returning a bank card before providing the withdrawn cash to prevent forgetful people leaving their card in the ATM. Another popular application of persuasion is 'gamification', the practice of embedding game elements in nongame contexts (McGonigal 2011). In the 2010s, a popular technology criticism was that social media platforms were persuasive by design, enabling internet addiction or compulsive use of digital media technologies (Alter 2017; Lanier 2018; Newport 2019) and preyed upon psychological weaknesses of end users that were originally identified and exploited by the gambling industry (see Schüll 2012). Scholars contend persuasive technologies further blur 
the boundary between the technological and human (Dorrestijn and Verbeek 2013; Verbeek 2009, 2011), reinforce the power of platform technologies (Stark 2018; Yeung 2017), are emblematic of wider institutional "traps" in society (Seaver 2019) and are complicit in the construction of mundane user digital practices (Jovicic, forthcoming).

I am not the first to assess the ethics of persuasive technology practice. Humanist critiques of persuasive technologies focus on the potency of the manipulation, claiming a line has been crossed when the design becomes too persuasive or coercive, and the subsequent threat to human agency and autonomy (Frischmann and Selinger 2018; Gray et al. 2018; Williams 2018). Instrumentalist critiques focus on how the persuasive technology is put to use (Berdichevsky and Neuenschwander 1999) and tend to come from persuasive technologists themselves. For example, popular persuasive technologists B.J. Fogg (2001) and Nir Eyal (2014) claim activities such as gambling or any product/service that harms the environment or animals are unethical applications of their persuasive technologies. Postphenomenologists claim persuasive technologists act ethically if they demonstrably anticipate and factor in a range of usages and effects of their inventions into the design process (Verbeek 2006, 2011). However, I depart from prevailing ethical enquiries of persuasive technologies and technologists who offer these valuable insights, and focus instead on hypothetical thought experiments, such as how the persuasive technology is put to use, or the robustness of the design process. From a humanist, instrumentalist or postphenomenological perspective, Boundless Mind appears to act ethically. In a self-published design manual titled Digital Behavioural Design(2018), Boundless Mind explicitly detail their methods of persuasion and their process of undertaking background checks of every commercial client before providing the API. 
Coming from a late Foucauldian perspective that views ethics as a self-directed exercise, I am interested in the influence of Boundless Mind and persuasive technology in the production of the user subject. Persuasive technology offers the potential to shape the conduct of end users. One reason this is possible is due to the application of the "psy" (psychology, psychiatry and psychoanalysis) sciences on human conduct beyond the laboratory. To understand the influence of the psy sciences first requires an overview of the Foucauldian concept of governmentality. Governmentality, or the governing of the human subject, was a concept that Foucault (1991) conceived to differentiate the operations of power in classical, liberal and neoliberal Western governments, where power was not always disseminated directly by the state, but manifested in knowledge, techniques and discourses and was internalised by individuals. Governmentality enabled Foucault to provide a framework to identify how individuals are connected to norms and related regimes of power. Early Foucault argued institutions such as prisons, hospitals and schools normalised individuals by inscribing regimes of truth and creating categories of abnormal and normal. He saw technologies of power as connecting "the norms of authorities to the motivating ideals [individuals] have of [them]selves" (Hook 2007, 246).

Rose (1998; 1999) argues the psy sciences extended governmentality, by not only normalising the idea of a human psychic interiority and exteriority, but also offering techniques to calculate the human psyche and therefore make it more knowable and manageable. The psy disciplines have been similarly influential in the formation of persuasive technology. The first published work concerning persuasive technology came from social psychologist B.J. Fogg (2001). Fogg is the founder of the Persuasive Technology Lab at Stanford University, where he formalised behavioural and social psychology research into a 
design model. Fogg was influenced by Albert Bandura's (1977) theory of social learning, a theory in psychology that considers behaviour a combination of observing stimuli and internal psychological processes. Fogg's (2001) key insight was the extent to which computers could operationalise basic principles of social psychology, which lead him to coin the term "captology" (an acronym for computers as persuasive technologies). Fogg (2001) believed that computers can persuade either as direct tools or passive intermediaries between actors by performing simulations, calculations or measurements to understand and elicit human motivations and behaviours. Captology "enabled human powers to be transformed into material that could provide the basis for calculation" (Rose 1999, 7). Notable graduates of the Persuasive Technology Lab include Mike Kreiger, the co-founder of Instagram, Evan Spiegel, the co-founder of Snapchat, and Tristan Harris, a former Google design ethicist and founder of the Center for Human Technology (Leslie 2016).

Crucially, persuasive technology design does not necessarily equate to manipulation. When analysing the use of the psy sciences to engineer demand for consumer products, Miller and Rose (2008) are careful to avoid implying that consumers have diminished agency. In their view, psychological techniques do not necessarily dominate or manipulate consumers, but mobilise them "by forming connections between human passions, hopes and anxieties, and very specific features of goods enmeshed in particular consumption practices" (Miller and Rose 2008, 115). Similarly, popular persuasive designer Nir Eyal (2014) claims to be able to influence end user behaviours by creating strong relations to consumer products. In his bestselling design manual, Eyal offers the "The Hook Model", which includes four phases of designing user habits: identifying external and internal triggers of behaviour; encouraging behaviours via the lure of reward; offering rewards at variable internals to 
generate a craving; and incentivising the user to invest in the product/service to improve their experience (Eyal 2014, 4-7). Other design models integrate affective aesthetics to enhance the emotive appeal of the end product (Norman 2004), while others lean more on behavioural data to measure the impact of the design and draw insights to refine the user experience (Wendel 2014). These persuasive models mobilise the end user to act by identifying and positioning certain relations to the user in subtly different ways. They have become formalised in User Experience methods such as A/B testing, which is a method to test the usability of a website or app by comparing two versions with slight differences against each other (Stark 2018). Because of these aforementioned design models, many apps or digital platforms use nudges, gamification, or other persuasive techniques to maximise user retention or generate behavioural outcomes for the benefit of the commercial client. In environments and economies where user attention is sought after (Crogan and Kinsley 2012; Wu 2016), persuasive technologies provide an edge for businesses over their competitors. Taken together, these models of behavioural change establish the application of persuasive technology as best practice in software development and design.

Boundless Mind claims to offer best-practice methods in mobilising the end user to commercial ends. Boundless Mind has published its own persuasive models and techniques to program user habits (Combs and Brown 2018). Their Cue-Action-Reward (CAR) Model follows previous behaviour change models by systematically instructing readers how to mobilise the end user by identifying cues, actions and rewards in relation to behaviour. Combs and Brown $(2018,21-25)$ claim to recognise the interior subjectivities of end users at an individual level. They combine neuroscience with behavioural science to create persuasive techniques such as "optimal information flow", which is the optimal ordering of information to 
elicit a desired user response, and "cognitive load balancing" to limit the mental work for the user in the Boundless Mind "Behavioural Design Toolbox". Moreover, on the Boundless Mind (n.d.) website, prospective commercial clients are informed about how Boundless mixes their behavioural expertise with software development. Readers are told that the brain is "programmable" and, to do so, you just the need the "code". The code is Boundless Mind's commercial persuasive technology: the Boundless API. The Boundless API combines machine learning protocols and neuroscience and applies them to a customer's app to increase user retention. Past clients include education and nutrition businesses who used the Boundless API to increase adherence to learning or dietary digital programmes (Shieber 2017). Given that users of such dietary or education apps are likely to be unaware the app has been optimised by the Boundless API, Boundless Mind are effectively mobilising the end user from a distance.

Despite a perception that the development of persuasive technology is correlated to the immobilisation of the end user by tethering them to their digital devices (Leslie 2016; Lewis 2017), popular persuasive technologists have acquired a 'therapeutic vocation' (Rose 1998) by repackaging their expertise as therapeutic solutions consumers can apply on themselves. Both Fogg and Eyal have turned their expertise on persuasive technology into self-help books, offering tips directly to consumers on how to develop everyday micro-habits (Fogg 2019) or become 'indistractable' (Eyal 2019) to the lures of social media. The therapeutic vocation is a reminder of the double-sided disciplinary nature of governmentality where a "social problem" of bad habits is viewed as fixable via habit-modification methods (Bennett et al. 2013; Pedwell 2017a). In the next section I demonstrate Boundless Mind's claim to act ethically by walking through their application Space. 


\subsection{The therapeutic authority of Boundless Mind and Space}

A scholar who observed the influence of scientific practice on Foucauldian ethics was Nikolas Rose. Rose $(1999,1998)$ analysed the rise of $20^{\text {th }}$ century psy disciplines into "therapeutic authorities". By offering unprecedented insights into human behaviour and interior states or subjectivities, the psy disciplines came to view the human subject as 'fixable', and their expertise as giving them the authority to administer therapeutic solutions to correct behaviours that they had contributed to constructing as abnormal (Miller and Rose 2008). By selling software products that claim to shape the behaviour of end users across conflicting vectors, Boundless Mind is a therapeutic authority of persuasive technology that have repositioned their expertise with a therapeutic solution for end users. The therapeutic solution is a free iOS and Android app and Chrome web browser extension called Space, which aims to help users disconnect from any app that the user wishes to use less. Moreover, Space reveals how persuasive technologists derive their therapeutic authority. My walkthrough of Space and analysis of Boundless Mind's therapeutic authority is organised around the two discrete steps of using the app: obtaining a replacement app icon and undertaking the therapy of a design technique called stimulus devaluation.

\subsubsection{Obtaining a replacement app icon}

When opening Space for the first time, users are taken through an onboarding process that provides information about the app in the wider context of persuasive technologies (Figure 5-A). The onboarding information reinforces Boundless Mind's expertise in persuasion and their view of the fixable subject. Users are informed that "it's not [their] fault [they are] hooked on apps" and that "Space puts you back in charge". The message of selfhelp is emblematic of a popular genre of literature in the 1970s that packaged seminal 
behaviourist B.F. Skinner's ideas in the format of self-control (Martin and Pear 2010; Watson and Tharp 2013). ${ }^{4}$ Yet the promise of Space is that the app (and not "you" the user) is the reason "you take back your brain." As behavioural and software experts, Boundless Mind claim an "internal truth" (Rose 1998) about the level of persuasion embedded in apps and the effects on users. Unless the user is well versed in behavioural science, neurotransmitters such as dopamine, or software development they are likely to interpret the onboarding information as "not an external truth-be it divine right or collective good-but an internal truth, one essential to each individual person over whom it is exercised" (Rose 1998, 91).
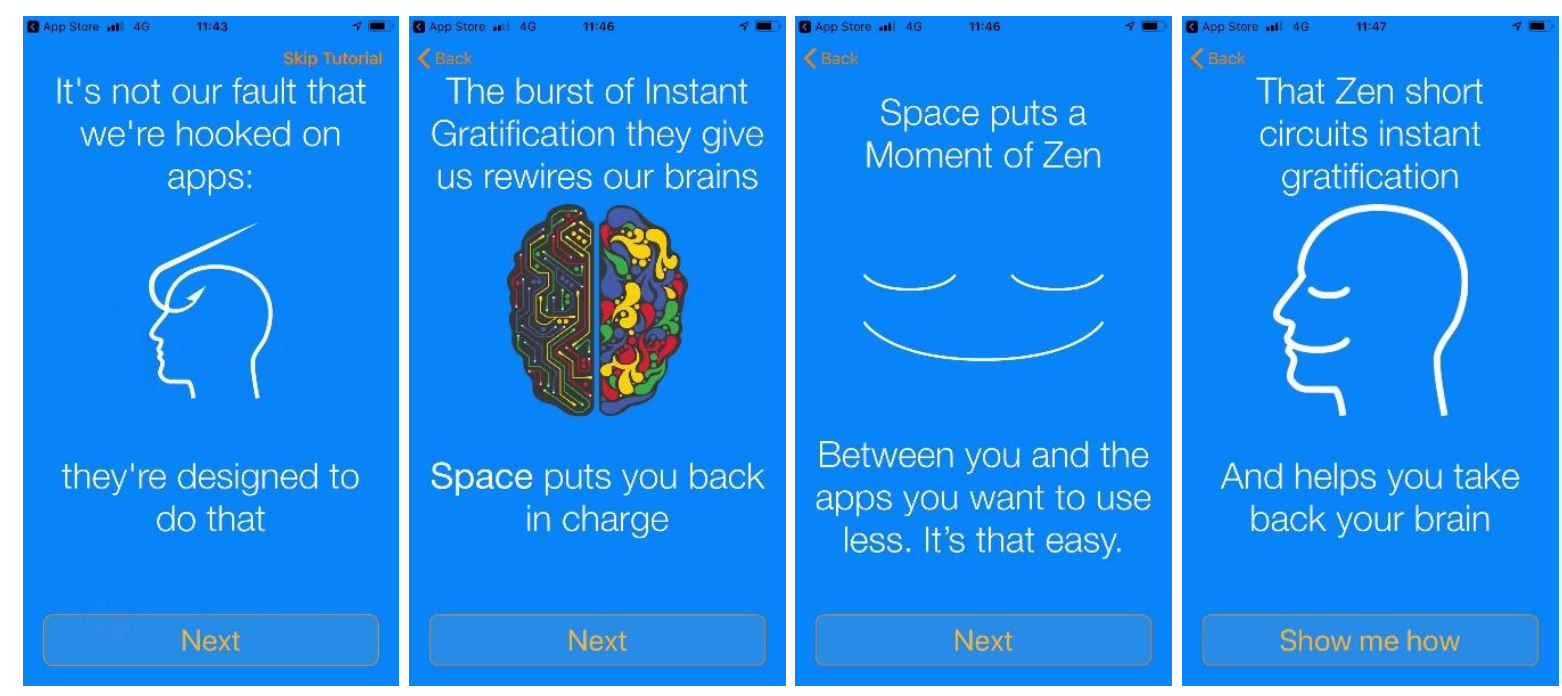

FIGURE 5-A: SPACE ONBOARDING PROCESS

authority. Therapeutic authorities are organisations with psychological capability and expertise to remedy recognised mental health deficits and deliver therapies (Miller and Rose 2008). However, if therapeutic authorities require official recognition of mental health deficits then there are doubts as to what exactly Boundless Mind is remedying. "Internet addiction" or

\footnotetext{
${ }^{4}$ Boundless Mind have an affinity for Skinner. Tim told me that Skinner "was a giant of a man academically, intellectually, but a deeply misunderstood man."
} 
"screen addiction" have not been officially recognised as a mental health deficit in the sixth revision of the Diagnostic and Statistical Manual of Mental Disorders (DSM-6), due to significant doubt concerning the causality between screen time and mental ill health (Orben, Dienlin, and Przybylski 2019). Yet the purview of therapeutic authorities goes beyond resolving mental health disorders that are grounded in empirical evidence. Rose $(1999,231)$ argues that therapeutic authorities have additional social power as they are also "sought when individuals feel unable to bear the obligations of selfhood", meaning the pressure to make strategic and justifiable decisions in a neoliberal society where day-to-day activities are subject to market forces and demand choices. One of the major concerns about persuasive technologies is their impact on end user productivity and attention spans (Carr 2011; Newport 2019; Soojung-Kim Pang 2013). The popular perception of a "digital distraction epidemic" (Twenge 2017) warrants the demand for a therapeutic authority like Boundless Mind.

For Space to work, users must first set up the intervention, which is achieved by obtaining a replacement icon. The homepage of Space is a directory of third party apps from which users commonly seek to disconnect (Figure 5-B). Apps are listed under categories of 'social', 'entertainment', and 'productivity', with users asked which app they would like to spend less time on. If the user wishes to use less of an app that is not listed, they can request that app to be added to the list. 


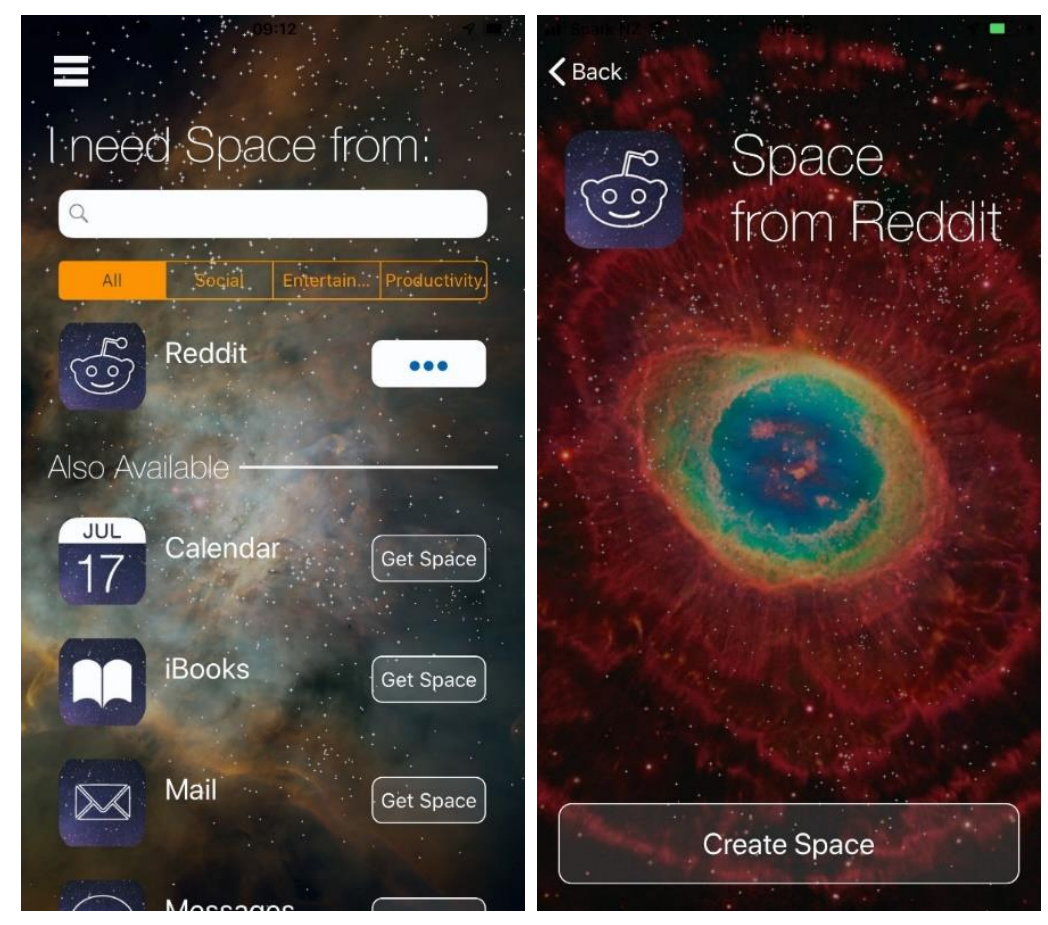

FIGURE 5-B: SPACE HOMEPAGE AND PROCESS TO GET 'SPACE'

Users choose which app they wish to use less by selecting the button 'Create Space'. In the iOS version of the app, doing so automatically opens the user's Safari mobile browser, taking the user to a website with the URL: https://canaveral.usedopamine.com (Figure 5-C). The fact that the website contains 'usedopamine' in the URL, reinforces Boundless Mind's position that a users' dopamine, or neurotransmitter that regulates mental and emotional responses, is available to instrumentalise to protect their attention. ${ }^{5}$ On the website the user is instructed on how to obtain a replacement icon for the app they previously selected. Here, Boundless Mind utilise an existing iOS functionality in Safari browsers called 'Add to Home Screen'. The 'Add to Home Screen' functionality is an iOS feature that enables users to create convenient shortcuts to favourite websites on their home screen. In this instance, Boundless Mind use the functionality of 'Add to Home Screen' to offer a replacement icon of the app the

\footnotetext{
${ }^{5}$ Further evidence of this point is the former name of Boundless Mind: Dopamine Labs (Shieber 2017).
} 
user has selected they want to use less. After adding the website to their home screen, a replacement icon appears that is visually distinct from the original app. The iconography and colours of the original app are redrawn into white wireframes and a space themed background. The reason why the replacement icon is not a direct replacement of the original app is because Boundless Mind does not want the user to remove the flagged app. If the user removes the original app, then Space stops working. Users are therefore instructed in the onboarding process to not uninstall the flagged app, but instead hide the original app icon in another screen or folder. However, relying upon the presence of the original app dilutes the impact of Space, comparable to giving a smoker a nicotine patch on the premise that the smoker must keep their cigarettes. Users are given the opportunity to customise the name of the replacement icon. Once they have completed these steps, the replacement icon appears on the desktop of the user's iPhone. The user can repeat these steps to obtain as many replacement icons as they would like.
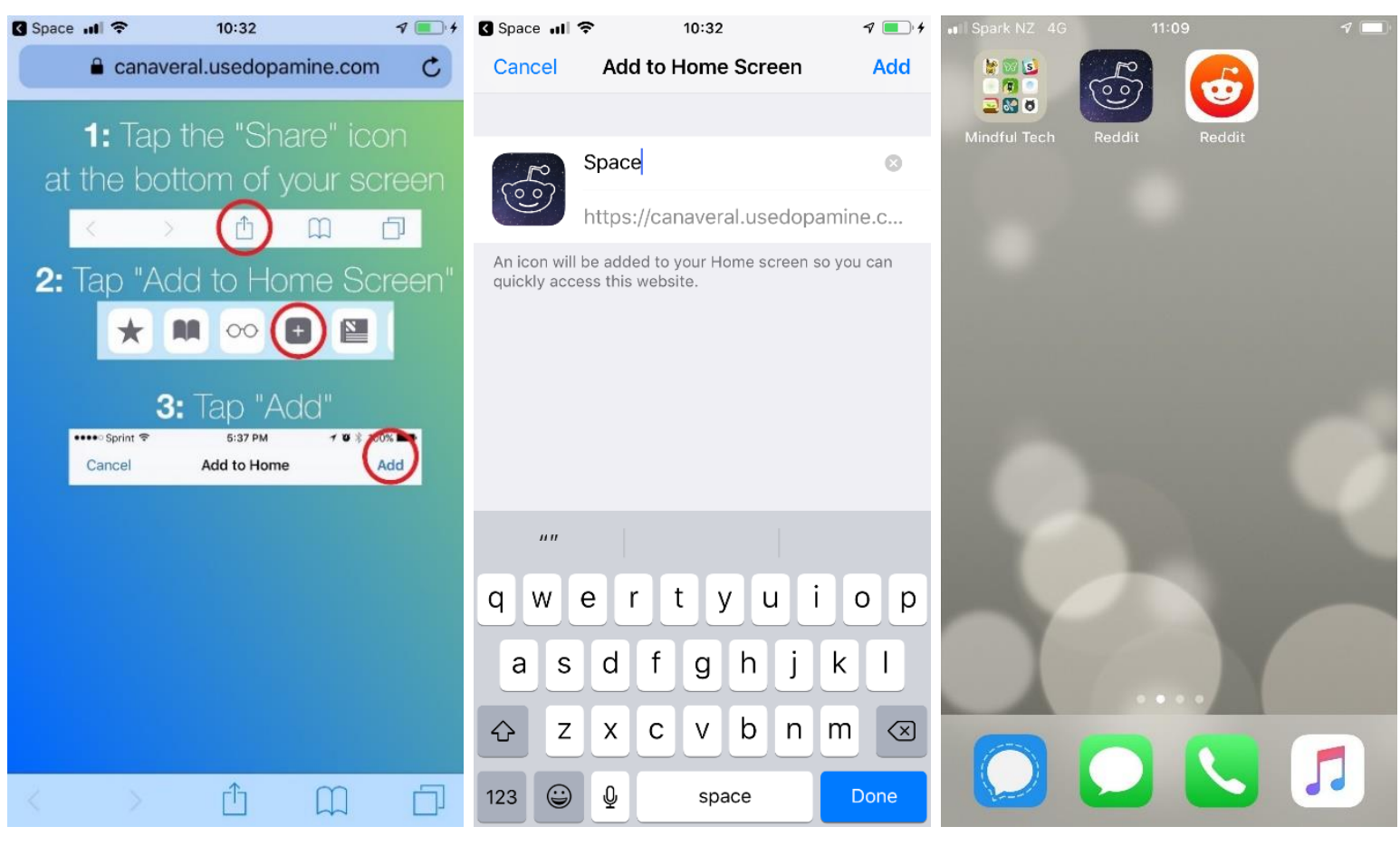
The replacement icon is intended to be used instead of the original app. The replacement icon therefore signifies the level of intimacy that Boundless Mind seeks to have with users, operating as an intermediary or invisible layer of software to intervene in instances where the user has highlighted a behaviour they wish to change. Furthermore, the issue of unconscious scrolling is framed as an issue to do with a user's interaction with persuasive technology, as opposed to persuasive technology itself. Tim explained his vision of Boundless Mind to me:

I want Boundless [Mind] to operate as the invisible layer of the net that glues all of that together. In the same way that Google gets to act as the invisible layer of the net around routing and search. I'd like us...to operate as a technology that we look back on as a turning point in a lot of the things that hurt really bad around behaviour, that we can walk back from some of these problems that totally suck.

Tim's comments reinforce that Space is not an app that is intended for either active or passive use. Rather, Space offers replacement icons that function as enhanced settings for smartphone users - a functionality that, by itself, is useless but, once set up in combination with other applications the user wants to limit the use of, provides an optimal process to avoid those apps.

\subsubsection{The therapy of stimulus devaluation}

Once the user has the replacement app on their desktop, the second step of Space becomes available; what Boundless Mind calls undertaking 'stimulus devaluation'. When selecting the replacement icon, the user is taken to a screen where they are prompted to take two deep breaths (Figure 5-D). The suggestion to take a breath is an explicit reversal of 
Apple's intended effect for the 'Add to Home Screen' functionality. Instead of offering a shortcut, Space provides a longer route to the original app, or a 'mindful' detour.

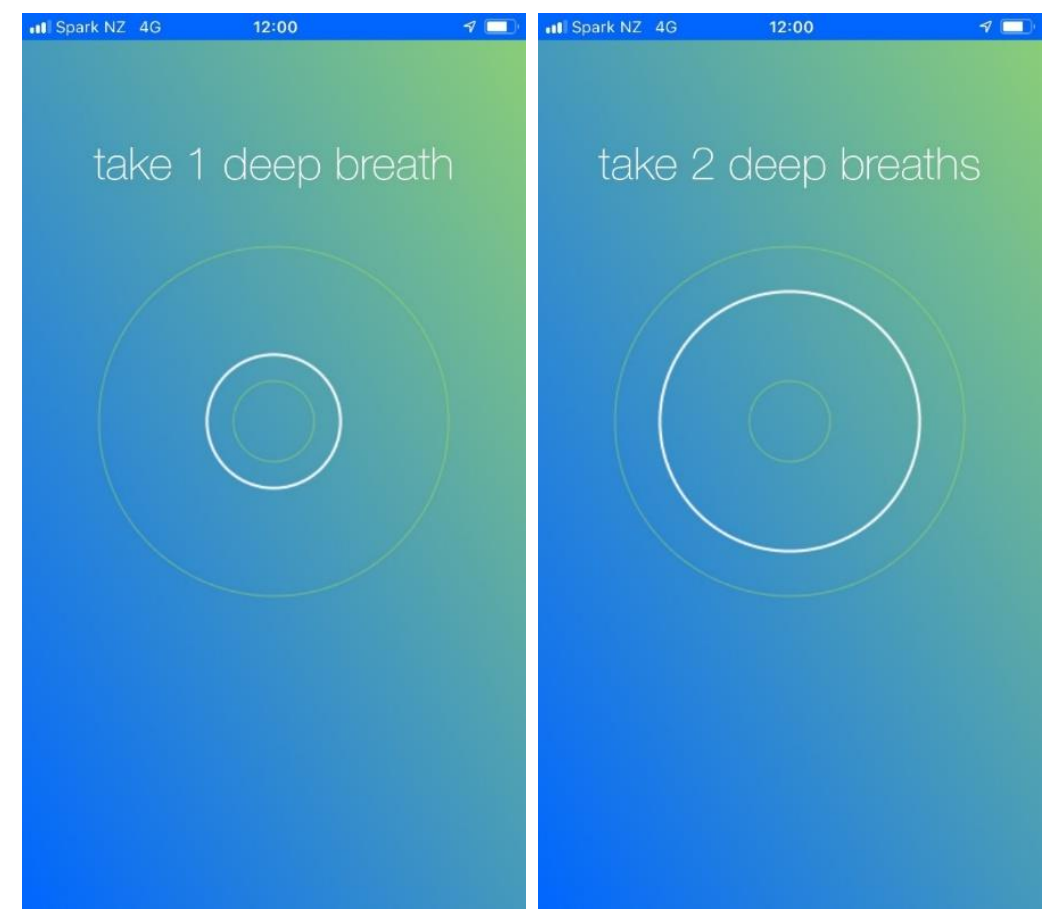

Figure 5-D: StimUlus dEVALUATION IN ACTION

The prompt for the user to take deep breaths is the psychological design technique stimulus devaluation. Stimulus devaluation draws upon behavioural science and is Boundless Mind's unique contribution to persuasive technology (Combs and Brown 2018). To explain the science behind the technique, Tim draws an analogy to the relationship between cigarette smoking and experiencing a nicotine rush:

The idea [is] if I gave you a cigarette, and you went to light the cigarette, and you took a puff of the cigarette but by some property of magic, you had to wait a few seconds between the breath in and the cigarette and getting the nicotine rush. And that happened every time and in fact the more you used the cigarette the longer you had to wait between taking the breath and getting the hit? You would still be free to use the cigarette, but you would be less interested in the cigarette. There starts becoming a gap between your action and the 
reinforcement for the action. If I received a notification from Facebook, which is a cue that I received and I take an action of opening up Facebook, and I have to wait between the action and the good feeling of seeing the notification that someone liked my post, my brain will have a hard time neurologically keeping the record of open Facebook, open Facebook, open Facebook running before it gets the dopamine hit. And if you start separating those, you weaken the connection between the trigger, the cue, and the action. The idea is how can we interject this delay that will still let someone do the action in a way that makes it less habit forming because the impact of reinforcement. It defames reinforcement.

Tim's description of stimulus devaluation suggests that the technique works on a similar premise to a self-control technique such as removing chocolate from a conveniently accessible location in one's home or worksite. Both technologies aim to break a habit the user has identified as problematic by increasing the delay between impulse and the reinforcing mechanism. In addition to breaking a habit, stimulus devaluation reverses the aim of prevailing persuasive technologies like Boundless Mind's CAR Model or Eyal's Hook Model. These prevailing models aim to create user habits by reinforcing the relationship between triggers and actions, while stimulus devaluation attempts the opposite and weakens those relationship by inserting a delay. Therefore, Space creates psychological barriers to the habitual or unconscious use of selected apps.

By using stimulus devaluation, Space restructures the process of opening flagged apps. Space does so by encouraging the user to access a different subjective state in order to curb their undesirable habits. The delay of stimulus devaluation is between eight to nine seconds, which is intended to shift the user from one subject position to another. At any point during stimulus devaluation, the user can exit from the app and avoid using the app they might have opened unconsciously. If the user waits out the detour screens, the user is taken to the original app. Space interprets the user passing through stimulus devaluation as the 
user intending to use the app consciously or mindfully, what behavioural economist Daniel Kahneman (2011) refers to as an effortful, slow or 'System Two' mode of decision making thinking. In contrast, 'System One' is an automatic, fast and often unconscious way of thinking (Kahneman 2011). System One thinking is an autonomous and efficient subject position, requiring little energy or attention, and is what the technique of stimulus devaluation tries to discourage. Space operates on the assumption that if users patiently wait out the detour, then the user intended to open the app, which constitutes acceptable use. By forcing the user to inhabit the System Two subject position Space is a:

[technology] of subjectivity [that exists] in a kind of symbiotic relationship with what one might term 'techniques of the self' the ways in which we are enabled, by means of the languages, criteria, and techniques offered to us, to act upon our bodies, souls, thoughts, and conduct in order to achieve happiness, wisdom, health, and fulfilment (Rose 1999, 11).

Rose's point is that it becomes very difficult to determine whether a technology such as Space is predominantly shaping the user subject (a technology of subjectivity, or what I referred to in chapter two as a technology of power) or if the user is constituting themselves (a technology of self). Instead of choosing one or the other, Rose opts to describe modern psy techniques as neoliberal governmentality tools. Neoliberal governmentality tools, according to Rose (1999) "govern [people] from a distance" because the techniques are not directly applied to citizens, but rather people willingly apply or consent to the techniques in the pursuit of specific goals, such as attaining happiness and self-fulfillment. Space is a neoliberal governmentality tool because it does not appear to infringe upon freedom but is the very thing that enables freedom-which in this case is freedom from online distractions and one's technology habits. The ambiguity of who conducts the ethical exercise has 
consequences for persuasive technology ethics, which I discuss in greater detail in the final section of this chapter.

\subsection{The obfuscation of persuasive technology power}

One of the implications of Space and the fixable self is that the habit-breaking app positions Boundless Mind as indispensable to self-directed ethical conduct. Foucault's imagination of a mode for subjects to be less governed "through reinventing subjectivity and autonomy within the existing social order" (Zamora 2019a, 263) reinforces the notion of a vulnerable neoliberal subject (Chandler and Reid 2016) that must continuously adapt to their surroundings and depend on external intervention from a persuasive technology. Foucault did argue that certain experts such as priests (and not individuals) became integral to the reinvention of the self, when describing alternative regimes of power such as pastoral power, which is the formalisation of a priest's knowledge of an individual's inner life via techniques of confession (Foucault [1976] 1990). But Foucault could not have anticipated that selfoptimisation culture would itself be normalised and institutionalised into everyday life (Binkley 2014) such as corporate or government wellness policies. The appeal of Space to users is the opportunity to apply behavioural expertise to curb their undesirable technology habits, a habit that by itself may be difficult to treat. When critically assessing nudges, Pedwell $(2017 b, 82)$ argues "responsibility is...delegated to 'choice architects' with the requisite knowledge and foresight to steer behaviour in appropriate directions". Space is a delegated authority to act on behalf of the user that intermediates "enterprise and submission, responsibility and discipline" (Schüll 2016, 12), both reinforcing and curtailing cultural ideals of personal responsibility. 
The underlying basis of nudges or persuasive technologies is that users do not always make the best decisions for themselves and reductions on individual autonomy are therefore justifiable. Yet the delegation of authority to Boundless Mind to fix the users' habits obfuscates the organisation's role in creating compelling and compulsive digital products in the first place. It is worth emphasising that the history of persuasive technology has more in common with the advertising industry than the psy disciplines. The original purpose of persuasive design was not to remedy perceived anti-social or undesirable behaviour like the psy therapies (Rose 1998), but to mobilise the end user to economically profitable ends. The success of persuasive technology in mobilising end users to economically profitable ends (e.g., spending more time online) have arguably compounded the perceived undesirable behaviour of mindless scrolling. The ethical perspective of Boundless Mind positions the organisation as providers of both the poison and the antidote to the issue of mindless user scrolling.

A persuasive technology ethics that predominantly relies upon expertise in user subjectivities is also silent with regards to the advances of power that the persuasive technology industry acquires. There is no account of the increasing asymmetrical relations between the persuasive industries (Stark 2018; Tufekci 2014; Yeung 2017; Zuboff 2019) and the end user. Yeung (2017) suggests that persuasive technologies become highly persuasive when combined with the engine of big data analytics. Big data has the potential to incorporate the user into feedback loops and personalise digital experiences which, in turn, can amplify the persuasiveness of technologies. In addition, the behavioural insights that Boundless Mind generate with Space renders more user subjectivity into calculable data: which apps users choose to disengage from, what times of the day users disconnect, or how 
successful their attempts to disconnect are. Data about users' technology habits could be valuable to a range of third party organisations and industries, such as employers, educators, marketers, or health insurers, raising significant privacy concerns. In fact, scholars have raised concerns about the fusing of big data and technologies of behaviour to shape consumer behaviour (Tufekci 2014; Yeung 2017). Not only could data about technology habits be used to govern social media users (Docherty 2020) or worker habits (Gregg 2018; Guyard and Kaun 2018), but could also limit access to credit and other schemes that determine certain behaviours to insinuate anti-social behaviour or financial precarity. The power and potential profit in operating Spacelie not only in the immediate nudge, but the broader behavioural insights that only a persuasive technology like Space can offer. For example, the German credit lender Kreditech claims to be able to assess borrowers' reliability by analysing over 6000 data points, including activity on social media platforms (Vasagar 2016). What will data on technology habits reveal about a user's trustworthiness or credit worthiness? Not having a social media presence-a type of passive disconnection-has been interpreted by recruiters in the past as socially abnormal behaviour (White 2012) which has the effect of normalising connectivity.

This chapter has critically assessed the activities of a persuasive technology organisation called Boundless Mind that claims to fix the undesirable smartphone user habit of mindless scrolling. By walking through their habit-breaking app Space, I have argued that the fixable self is driven from persuasive technologists' expertise in user subjectivity and capability in mobilising the user to economically profitable ends. I have also suggested that the fixable self obfuscates the role of Boundless Mind in creating compelling environments in 
the first place. However, there is arguably a bigger-and culturally specific-influence on the manufacture of disconnection that I discuss in the next chapter. 


\section{The intentional self and the dropouts of Silicon Valley}

The United States' technology industry has a famous and unique culture. Originating from the Silicon Valley in Northern California, the technology industry is a melting pot of technological utopianism, libertarianism, and eco-activism. In this chapter, I investigate the influence of Silicon Valley culture on the manufacture of disconnection. Foucault ([1984] 1997, 291) did not believe that people developed critical self relations in isolation, but via "models that he finds in his culture and are proposed, suggested, imposed upon him by his culture, his society, and his social group." I focus on a specific subculture of Silicon Valley that is countercultural, or at least was countercultural at the time it emerged in the 1960s. By countercultural I mean subcultural values, practices and norms of behaviour that differ significantly from those in conventional society (Wright 2017). The technology industry is steeped in subcultures, such as the open software movement (Kelty 2009) hacking culture (Olson 2013) and experimental wellness subcultures (English-Lueck 2010) but in this chapter I use counterculture to mean the Silicon Valley subculture of dropping out from conventional society. Dropout culture is bigger than Silicon Valley, perhaps most famously epitomised by 1960s countercultural icon Timothy Leary (1999), whose popular catchphrase "turn on, tune in, drop out" called for people to resist media technologies and take recreational psychedelic drugs instead. ${ }^{1}$ While many Silicon Valley dropouts took drugs, this chapter focuses on how Silicon Valley dropouts retreated from society to create alternative environments for others to inhabit and partake in intentional or goal-orientated living.

\footnotetext{
${ }^{1}$ Leary (1999) first popularised the catchphrase in 1966 at anti-war rallies in San Francisco. Leary revealed in a 1988 interview with Neil Strauss (2011) that he was given the slogan by none other than the famous media theorist Marshall McLuhan.
} 
It is over fifty years since the original dropouts retreated from conventional society; today, large groups of people retreat from what we might call digita/society. By digital society I refer to societies where digital media technologies provide core political, social, economic and cultural infrastructure to enable people to conduct and extend their lives across space and time (Lupton 2014). My interpretation of a digital society is not to suggest there is a distinct cyberspace from physical reality and reinforce digital dualism (Jurgenson 2012). Instead, I use digital society to suggest a connection between the original 1960s and 70s dropouts who retreated from mainstream societal structures and today's digital dropouts who reject conventional usages of digital media technologies. A central argument of this chapter is that the countercultural ethos of intentionality underlies the design and use of digital media technologies, producing what I refer to as the 'intentional self'.

One technology of disconnection that produces the intentional self is Siempo. I learnt from my interview with Siempo Chief Executive Officer Andrew Dunn that Siempo is a portmanteau of the Latin words sempre (always) and tiempo (time), to denote 'a mindful use of one's time'. This chapter consists of two parts; the first part offers a brief history of dropout counterculture in the Silicon Valley to identify the origins of the intentional self and provide a cultural context to Siempo. Drawing upon Fred Turner's (2006) seminal text From Counterculture to Cyberculture, I argue the practice of dropping out from mainstream society to create and partake in alternative social structures reinforced design, technology and personal transformation as integral to addressing social issues. Today, dropping out lives on in the form of countercultural events such as Burning Man and the health practice of digital detoxes. In the second part, I walk through Siempo to highlight expectations of use and how the intentional self is produced. I identify features that use countercultural and health ideals 
to encourage the user to aspirational ends and to self-discipline their digital activities. I conclude by suggesting that the intentional self is indicative of a broader neoliberal politics where authentic lifestyle and consumerist practices are positioned as the ideal means to solve societal problems.

\subsection{Silicon Valley and intentional communities}

The practice of 'intentionality', or goal-orientated living, is an idea that is central to Silicon Valley dropout culture. So far, no studies have discussed intentionality in relation to the ethos of Silicon Valley and their technological inventions. Previous cultural analyses of Silicon Valley have labelled the technology industry as a hotbed of hypermasculinity (Chang 2018), technological utopianism (Levina and Hasinoff 2016; Morozov 2014) or libertarianism (Taplin 2017), suggesting paternalistic and technological deterministic overtones to Silicon Valley culture along with an aversion for unionisation, greater political oversight or regulation. In contrast to these studies, I argue dropout elements to Silicon Valley culture are informing the manufacture of disconnection. The following section provides an overview of Silicon Valley dropout counterculture and suggests a connection to modern and popular self-care practices such as digital detoxes and mindfulness.

\section{1. 1. Dropping out and alternative lifestyles: New Communalists and Burning Man}

In the late 1960s, tens of thousands of Northern Californians retreated from society to live in alternative communities and seek social cohesion and optimal methods of living. In a cultural history of the Silicon Valley, Fred Turner (2006) christens a loosely affiliated group of Californian dropouts who retreated from mainstream American society in the 1960s and 70s as the New Communalists. These New Communalists sought communal living in rural areas 
as a response to the materialism of the 1950s and alienation of life during the Cold War. Many New Communalists were technologists, building high-tech geodesic domes, and connected to each other via informational networks such as Stewart Brand's Whole Earth Catalogue. The Whole Earth Catalogue presented tools and technologies to help New Communalists live self-reliant and spiritual lifestyles. According to Turner (2006) the New Communalists were largely apolitical, which distinguished them from the New Left, another countercultural group that emerged in the 1960s. Both the New Communalists and the New Left sought transformative societal change, but while the New Left was a traditional political movement that published manifestos, organised rallies and marched, the New Communalists turned "toward technology and the transformation of consciousness as the primary sources of social change" (Turner 2006, 4). On a similar note, cultural critical Christopher Lasch (1979, 4) criticises the New Communalists for their obsession with "psychic self-improvement." Turner (2006) positions the New Communalists as the cultural backbone of Silicon Valley cyberculture with links to Californian ideology: the unwavering belief in the emancipatory potential of technology (Barbrook and Cameron 1996).

Today's equivalent to New Communalism might be found in so-called 'intentional communities'. Although intentionality is well discussed in academic fields such as technology philosophy and phenomenology, as a folk concept it is under scrutinised. In folk communities, intentionality generally refers to goal-orientated behaviour (Cushman 2015) or conduct entailing belief, desire, intention, awareness, or skill (Malle and Knobe 1997). According to Shenker $(2010,6)$ an intentional community is "a relatively small group of people who have created a whole way of life for the attainment of a certain set of goals." What distinguishes intentional communities from typical tribes, villages or other clusters that spontaneously 
emerge over the years, is that an intentional community is a determined group of individuals who come together to create an entire way of life. Intentional communities aim to achieve cohesive social integration, with some communities using behavioural design to organise and create governance and social structures. Twin Oaks Community is one example of such a community; inspired by Skinner's Walden Two (1948), Twin Oaks used behaviourist techniques to design its social systems. Twin Oaks no longer uses behaviourism and now calls itself an intentional community, reinforcing the link between design practices and countercultural ideals of intentionality. Odell (2019) suggests communities such as Twin Oaks are ahistorical, largely insular from political change and difference, and can be interpreted as a form of 'lifestyle libertarianism': the practice of self-governance that is free from outside interference.

Given the prevalence of libertarianism in the technology industry (Taplin 2017), it is perhaps unsurprising that the language of intentionality has crept into technology culture. In a profile of notable design ethicist and Center for Humane Technology leader Tristan Harris, Bosker (2016) reports that Harris lives in an intentional community house in San Francisco with other entrepreneurs. Instead of retreating to a commune away from society, Harris appears to have implemented ideas of intentionality into his home and everyday practices. Intentionality has also provided a cultural gloss to age-old activities such as intentional eating (deBruler 2017) or intentional teaching (Houghton 2013), transforming intentionality into a consumer practice. Moreover, Burning Man, arguably the apotheosis of countercultural hedonism, is viewed by some attendees as an intentional event. Brian Doherty describes Burning Man as a "true experiment in intentional creative community" (qtd. in Steinhauer 2018), and there are stories of Burning Man patrons ("Burners") transitioning to more 
permanent intentional communities (UPLIFT 2015). Throughout the week-long party, socalled Burners integrate creativity and performance into local economies, share resources, and partake in conscious or deliberate behaviour (Turner 2009).

Events such as Burning Man situate counterculture as consumable to new audiences. Zandbergen $(2012,364)$ notes how Silicon Valley countercultural communities in the 1960s and 70s endorsed a type of participatory consumerism that "liberated emancipated commodities from imposed conventions about their use." While Burning Man has typically been framed as rejecting conventional consumerism (Gilmore and Van Proyen 2005), Kozinets $(2002,36)$ contends the rituals of exchanging gifts are attempts to reinvigorate "consumption into a liberatory pursuit distanced from market logics". The appeal of Burning Man is the contrast it provides to "conformist and alienating superficiality of media-saturated and consumerism-fuelled, corporate capitalist mainstream culture" (Gauthier 2013, 144). What can be gleaned from these studies is that dropout culture does not wholly reject consumerism, but rather attempts to disrupt consumerism from conventional market logics. As McGuigan (2009) notes, countercultures serve an important function to late forms of capitalism by repackaging commodities to appeal to younger distinctive demographics. As local and temporary as countercultural modes of consumption at Burning Man may be, such disruption broadens the scope for consumerism to capture new varieties of individual authenticity.

Burning Man also has a specific appeal to technologists. According to Turner (2009), Google's founders, Larry Page and Sergey Brin, have attended Burning Man since 1999 and encourage Google team members to attend to find artistic inspiration for their work projects. To Google, Burning Man presents an untapped common of ideals, processes, projects and 
artists that is beneficial to creative work (Turner 2009). By meshing labour processes and culture in such a way, Burning Man offers an optimal cultural scaffolding for new media production. Turner writes (2009, 75-76):

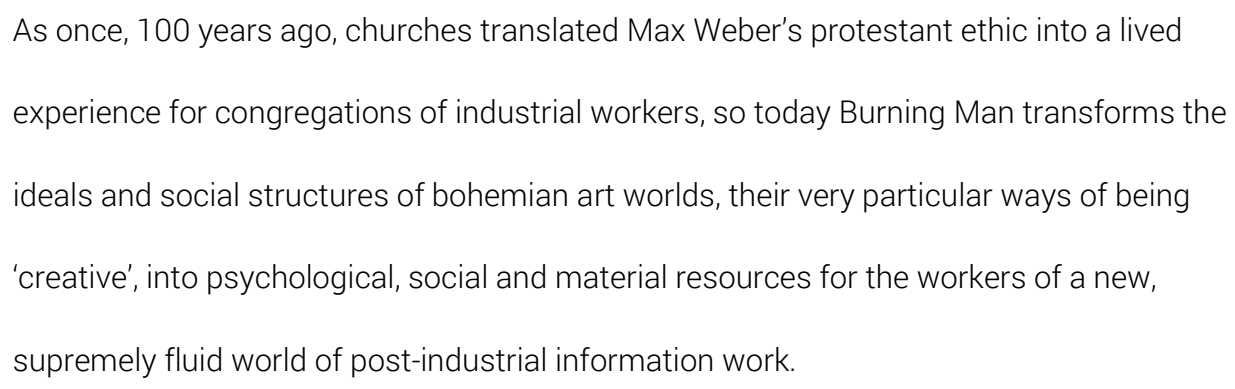

Google workers at Burning Man and intentional community houses in San Francisco indicate a trend in Silicon Valley whereby an idea or practice is transplanted from its countercultural origins and instrumentalised for work or everyday practice. New Communalism, intentional communities, and Burning Man all reinforce the notion that dropping out and acting with intention is not merely a practice of disconnecting from mainstream society, but generative of new ways of working and living.

\subsubsection{Dropping out goes mainstream: digital detoxes and self-help literature}

Today, people drop out from the digital milieu. Platforms such as Facebook have lost appeal to certain users due to a perception that they foreground anti-social culture and behaviour, spread disinformation, as well as perpetuate outrage and body shaming culture (Vaidhyanathan 2018). A popular type of digital dropouts are periods of abstinence from digital media technologies, otherwise known as digital detoxes (Syvertsen and Enli 2019). Digital detoxes are increasingly popular activities. In the summer of 2016, over 15 million British internet users undertook a digital detox (Ofcom 2016), and off-the-grid holidays are on the rise (Cartwright 2018). Digital detoxes have links to previous iterations of dropout 
culture-for example, the digital detox summer retreat Camp Grounded in Texas was created by former Burner Levi Felix (Fish 2017). In an ethnographic study of Camp Grounded, Fish (2017) suggests digital detox camps merge the principles of New Communalism with postdotcom entrepreneurism and eco-spiritualism of the 2000s anchored together by the individual pursuit for wellness.

A digital detox frames a digital dropout as a health-related activity, with social media negatively seen as unhealthy or providing a superficial experience (Sutton 2017). The utilisation of a health frame reinforces a neoliberal ideology that Fish (2017) calls 'digital healthism', which positions the individual as responsible for managing their own digital consumption. Digital healthism suggests how digital-free retreats have simultaneously become a luxury or "corporate amenity like a gym or a cafeteria" (Odell 2019, 33), and also a public health initiative. Digital detox campaigns such as Scroll Free September (Royal Society for Public Health 2018) and Pause (Capsana 2019) frame digital dropouts as activities individuals can undertake to better manage their digital habits. When outlined as a public health issue, a digital detox loses some of the original counterculture lustre in exchange for normative legitimacy. Scrol/ Free September and Pause still reinforce digital healthism by encouraging people to undertake self-care practices of disconnection, but aim to make such practices accessible to all interested citizens.

Self-help literature further embeds a digital dropout within popular culture. Popular authors such as Carl Newport (2019) call for a lifestyle of 'digital minimalism' that requires taking stock of digital dependencies, decluttering the digital inventory and deliberately choosing what to pay attention to or not. Other authors call for media diets (Levy 2016; Sieberg 2011) or aim to empower readers to master their digital environment via 'mindful' or 
contemplative techniques (Gordhammer 2009; Rheingold 2012; Soojung-Kim Pang 2013; Chade-Meng 2012). The self-care techniques offered in these texts encourage readers to annotate and more actively self-reflect upon their digital habits.

Similar to the New Communalists, modern day Silicon Valley dropout culture does not reject media technologies per se but instead rejects how media technologies should be conventionally used. To encourage alternative technology consumption or usage, ambient or minimalist ideals are influencing the design of technologies. Instilling digital media interfaces with a contemplative spirit is the aim of calm technology (Weiser and Seely Brown 1995) or calm design (Case 2015) and a subfield of human-computer interaction ( $\mathrm{HCl}$ ) called 'positive computing'-a combination of $\mathrm{HCl}$ and positive psychology (Calvo and Peters 2014). Calm technology or design, positive computing, and organisations such as the Center for Humane Technology are the latest incubators of the deep-seated Silicon Valley cultural belief that alternative lifestyle practices and revamped technology and design can address social issues and maximise human potential (Zandbergen 2012).

\subsection{The intentional interface of Siempo}

An example of a technology that addresses the issue of the distracting internet is an Android app and Chrome browser extension called Siempo. The purpose of Siempo is to disconnect or drop the end user out from common features of the smartphone that are distracting or encourage mindless phone usage. Features of the smartphone that are considered by Siempo to be potentially distracting are the interface and notifications. Siempo integrates the countercultural ideal of intentionality to reorganise these features and package disconnection as a consumer product for self-care. 


\subsubsection{Intentional packaging of the interface}

To encourage the user to undertake intentional or goal-orientated online behaviour, Siempo comprehensively reorganises and redraws the Android smartphone interface (Figure 6-A). Once the user installs the app they must provide consent to allow Siempo to overhaul the standard Android home screen. ${ }^{2}$ If consent is given, all colours and app branding of the interface are stripped away, replaced by a pallid grey background. The home screen is left largely blank except for a question field prompting users to consider their intention. The white space and decluttered interface of Siempo echoes the minimalist principles of calm design (Case 2015) and is the basis of an inconspicuous aesthetic that Portwood-Stacer (2012a) argues can influence taste in media, and in turn, drive media rejection.

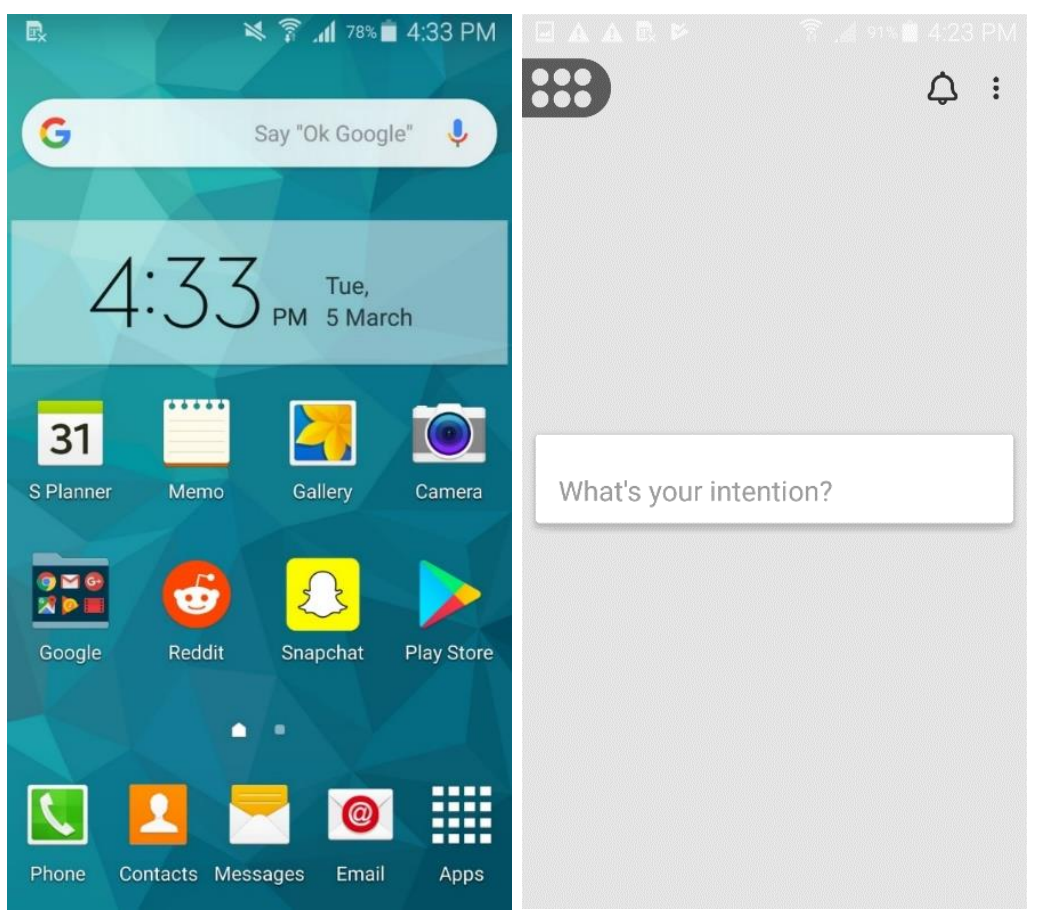

FIGURE 6-A: THE HOME SCREEN OF A SAMSUNG GALAXY 5 BEFORE AND AFTER INSTALLING SIEMPO

\footnotetext{
${ }^{2}$ Customization of the home screen is the primary functionality of Android launcher applications (Beal n.d.). Launcher apps are Android specific technologies which distinguishes Android as an open software development platform to Apple's more tightly controlled app ecosystem. As Siempo is incompatible with Apple's app development policy, Siempo is therefore only possible on Android devices.
} 
The aim of the Siempo interface is to disincentivise smartphone usage, which bears similarities to the goal of plain-packaged cigarettes. In New Zealand and Australia, cigarettes are packaged with uniform and dull aesthetics to reduce the appeal of smoking. Addiction experts justify plain packaging on the basis that smoking is unhealthy and the use of branding, colours and imagery on cigarette packets increase the appeal of smoking (Dewe, Ogden, and Coyle 2013; Wakefield et al. 2013). The plainness of the Siempo interface draws upon similar behavioural psychology to make the smartphone less appealing. Unlike plain cigarette packets, however, Siempo does not aim to prevent a behaviour in general but encourages the user to be intentional with the way they use their phone. Users are prompted to set their intention via an open field text box (Figure 6-A). The box asks: "What's your intention?" Some examples are provided, including: "spend more time with family," "eat healthy foods," and "keep my phone locked". Once users have set their intention, this text stands out on the screen (Figure 6-B).

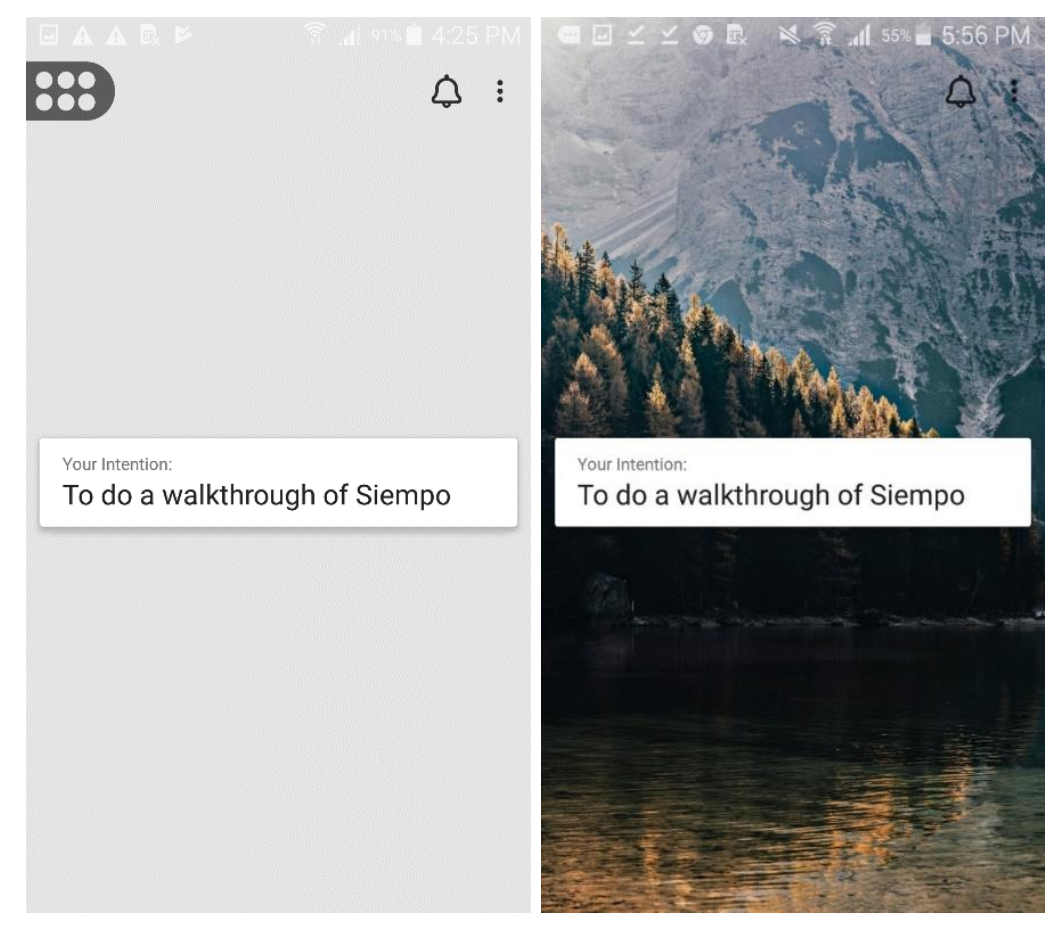


If the user wishes to, they can circumvent the plainness of the intentional interface and customise the background image. There are no restrictions on what images can be chosen; if the user wanted to, they could select a lurid image or a picture that promotes an unsocial or, ironically, pro-social media behaviour for their background. The implication is that the plainness of the interface is not of primary importance; the bigger priority is that the user sets an intention. Dunn explained to me that the purpose of the intention prompt is to gently nudge the end user to reflect on their smartphone use:

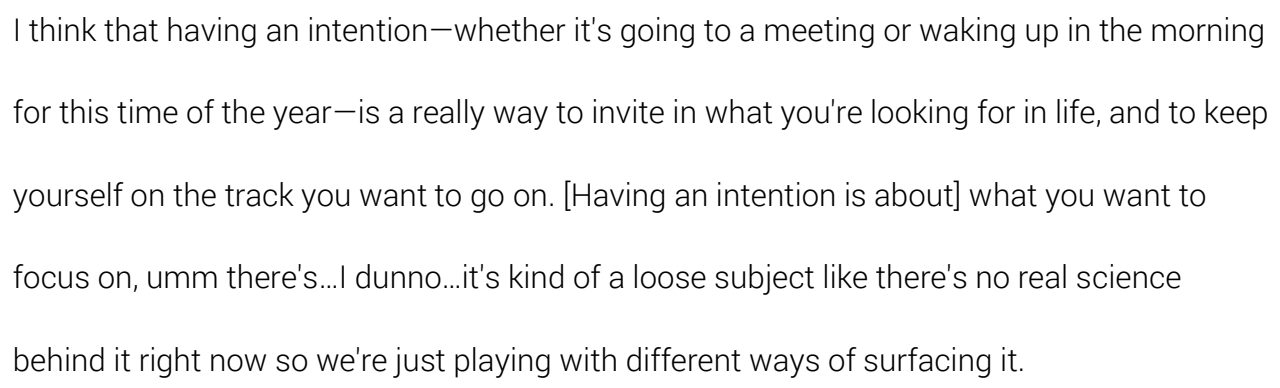

Dunn's comments reinforce that the benefits of setting an intention are not based on empirical evidence, distinguishing Siempo from the plain packaging of cigarette packets.

There is scientific evidence that cigarette smoking is harmful (Das 2003) and, in New Zealand and Australia, the plain packaging of cigarettes is just one public health policy intervention alongside policies of pricing hikes and the hiding of products that share the goal of reducing smoking uptake. Plain packaging is a regulatory intervention with clear policy objectives grounded in empirical evidence that smoking cigarettes is harmful (Dewe, Ogden, and Coyle 2013). More broadly, the field of health psychology justifies manipulating people to partake in intentional behaviour in situations to address gambling or obesity (Churchill, Jessop, and Sparks 2008; Ohtomo 2013). In contrast, the debate about whether social media, or digital screens are harmful is highly contentious. For example, while some studies claim a heavy use of social media can negatively affect users who are socially anxious and prone to compare 
themselves to their peers (Jiang and Ngien 2020; Lee-Won, Herzog, and Gwan Park 2015; Reer, Tang, and Quandt 2019), other studies report that socially anxious users report feeling more in control when communicating online due to an increased sense of anonymity (Shepherd and Edelmann 2005). The variability of users' psychosocial profiles and the multiplicities of how social media can be used may be why any causality between screens, social media and mental ill health for ordinary users has not been established (Orben, Dienlin, and Przybylski 2019). Any claims by Siempo that their intentional interface will generally improve the wellbeing of its users (Figure 6-C at http://www.getsiempo.com/) are not grounded in empirical evidence.

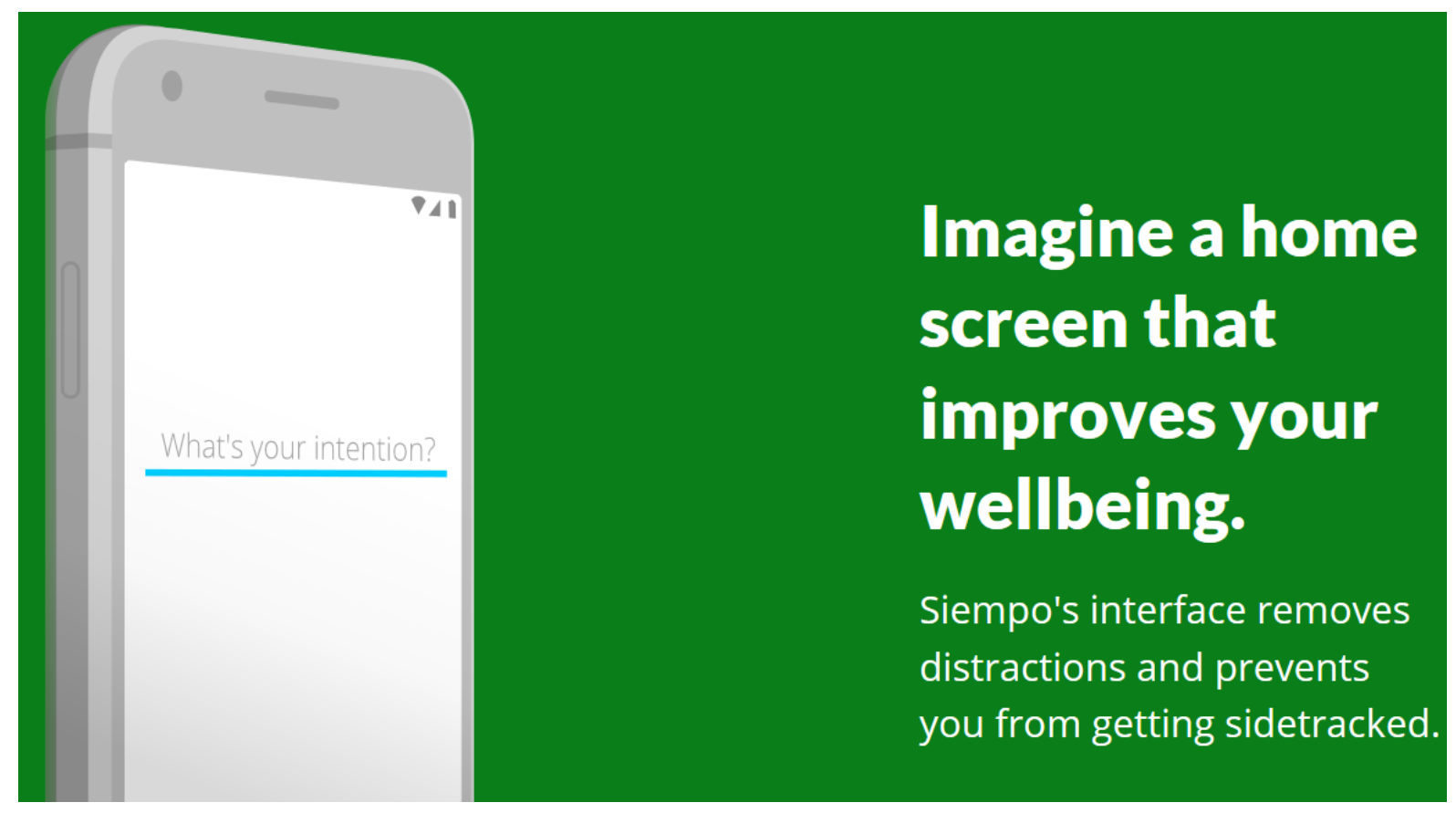

FIGURE 6-C: A SCREENSHOT FROM THE SIEMPO WEBSITE

Instead, Dunn's confidence that there are benefits to setting an intention derives from a pseudo-scientific Silicon Valley dropout culture. Siempo draws upon the ethos of intentional communities and Burning Man to encode the ideal of goal-orientated behaviour within the smartphone interface. Whereas alternative communities became intentional by rejecting 
conventional social structures to design their own systems and rules based on shared social values, Siempo rejects the conventional structure of the smartphone interface in favour of a rigid design that encourages the individual user to inhabit the intentional subject position and act upon their intentions. Users are reminded of their intention every time they unlock their phone, as well as when they swipe to additional screens. The creation of an intentional interface by Siempo suggests that Silicon Valley counterculture does not only inform Silicon Valley production processes (Turner 2009), but also the design of interfaces. Users are not entirely disconnected from the internet, but are instead encouraged to drop out from any distractions that could deter them from their set intention. The intentional interface of Siempo turns the smartphone into a kind of digital commune-for-one where a focused and purposeful life can be realised.

However, the intentional self produced by the Siempo interface appears to fall foul of the 'designer's fallacy'-a deistic belief that the designer has a God-like power to design into a technology all intended purposes and usages (Idhe 2008). It is doubtful that any user would want to use their smartphone intentionally all the time; yet there appears to be a presumption by Siempo that the user should always have an intention, an unlikely scenario given intentionality is only one component that motivates behaviour alongside willingness and habit (Churchill, Jessop, and Sparks 2008; Ohtomo 2013). By frequently requesting the user set their intention, Siempo encourages purposeful behaviour and risks foreclosing any spontaneous or unintentional usages of the smartphone. The effects of constant goal setting is the normalisation of self-governance, similar to other smartphone monitoring apps that impose "endless micro-project management, transforming downtime into something structured, obedient, and explicitly purposeful" (Beattie 2018b). On Siempo, there is less room 
for idle smartphone play or digital wayfaring; the implication for users is to actively manage all activities as part of a wider intention. As all human behaviour cannot be intentional, the actual effect of the Siempo interface is to nudge users to self-manage their digital consumption and aspire to healthier, productive or otherwise self-optimal modes of living that are determined by Siempo. The intentional self may disconnect users from unconscious smartphone behaviour, but that disconnection brings them closer to normalised regimes of self-optimisation (Binkley 2014). In addition, the intentional self resists the attention economy via governmentality techniques that are more overt than how Foucault $(1982,136)$ envisaged governmentality when famously defining the operation of power as the "conduct of conduct." As Foucault $(1982,221)$ explained:

To 'conduct' is at the same time to 'lead' others (according to mechanisms of coercion which are, to varying degrees, strict) and a way of behaving within a more or less open field of possibilities. The exercise of power consists in guiding the possibility of conduct and putting in order the possible outcome. (emphasis added)

Siempo and the intentional self lead the user via a persuasive interface that attempts to do more than merely guide the possibility of user activities. The international packaging of the interface actively works to disincentivise the use of certain apps that are considered by Siempo to be typically accessed unintentionally.

\subsubsection{Tidying the app menu}

Siempo also reorganises the inventory of apps on the smartphone. When users swipe left from the home screen for the first time, they are welcomed to a "healthier app menu!" and prompted to arrange their most helpful apps on this screen. (Figure 6-D). Apps that are considered 'tools'-maps, rideshare apps, or the camera-are foregrounded to positions of 
convenient access in the app inventory. If a category (e.g., "wellness") has not been assigned to an app, then the user is prompted to do so. For example, an app that the user could assign to 'wellness' could be the meditative app Headspace.

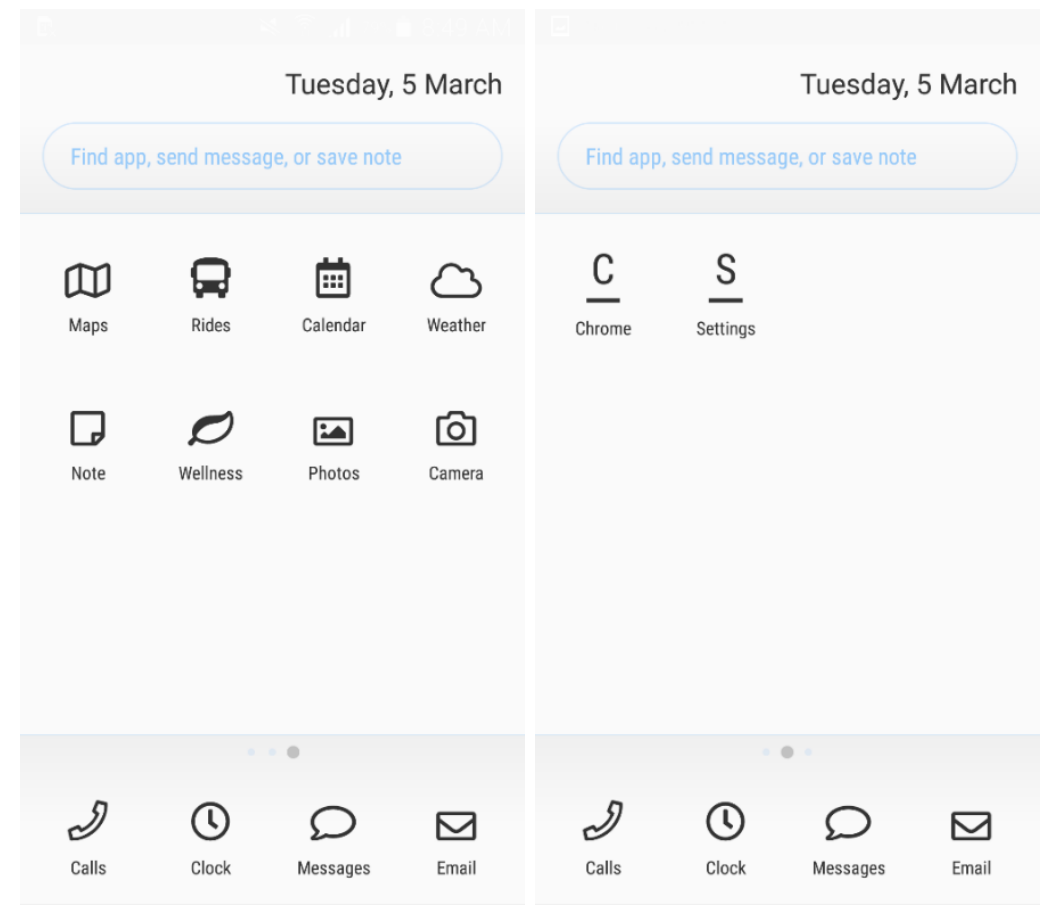

FIGURE 6-D: TOOLS AND 'FREQUENTLY USED APPS' OF THE SIEMPO INTERFACE

The foregrounding of utility and wellness apps is a reminder of the recent minimalist lifestyle trend that encourages readers to remove any items from their homes or workspaces that are neither functional nor elicit delight (Kondō 2014; Newport 2019). The tidy app menu of Siempo encourages a similar cleanout of the digital inventory, and promotes a similar lifestyle minimalism of the digital home, where ideals and practices of consumption and productivity are simultaneously rejected and celebrated through self-growth narratives (Meissner 2019).

'Frequently used apps' that are deemed 'non-distracting' are pushed onto a second screen (Figure 6-E) which includes the internet browser app Chrome. Siempo does not 
disclose what criteria are used to determine what apps are 'non-distracting'; this decision is largely left to the user. Instructions are provided on how to add any other non-distracting apps if the user chooses to. That Siempo is happy to support Chrome-a Google-owned app that tracks users to capture their data (Zuboff 2019)-suggests that the tidy app menu is not designed to protect the user from surveillance activities or enhance privacy, but instead to eliminate perceived distractions. Researchers who examine the link between attention and wellbeing argue that distractions can contribute towards feelings of purposelessness and unhappiness (Dolan 2014) and preclude deep cognitive thought practices that enable people to define their goals and values in the first place (Williams 2018). Dunn considers a pivotal moment in their life was when they were travelling overseas and were disconnected from the internet:

So, when I got to India, a month after I got there I travelled by myself for a week. That was my first time really travelling solo for more than a few hours, and the first night I got to this one city and I found this hostel and I put my shit down and after twelve hours of really draining travel at night, and I was just alone. Further away from anyone I had ever been - no electronics, no Wi-Fi. Which was unintentional - I had them with me, they were just dead, and there was no Wi-Fi at this hostel. I had a notebook with me and I had this three-dollar hostel room. It was the first time that I had this silence and pause and this space to really examine myself and I feel like I looked down at my hands and I was like who am I? what have I been doing in the last ten years? It was this surreal experience where I suddenly felt clear and creative and free and present and alive. It was like I was learning about myself for the first time and I had snapped out of this hypnosis of tech, that I had been under for a decade. It was like holy shit! now what? what's important to me, what happened, what do I want?

Dunn attributes the opportunity for deep revelation and self-reflection to being disconnected from the internet. The tidy app menu attempts to offer the same experience by foregrounding 
wellness and note-taking apps to encourage users to partake in introspective activities on a regular basis. Apps that are considered to be the most distracting and therefore injurious to self-discovery, are positioned the furthest away on a third screen (Figure 6-E).

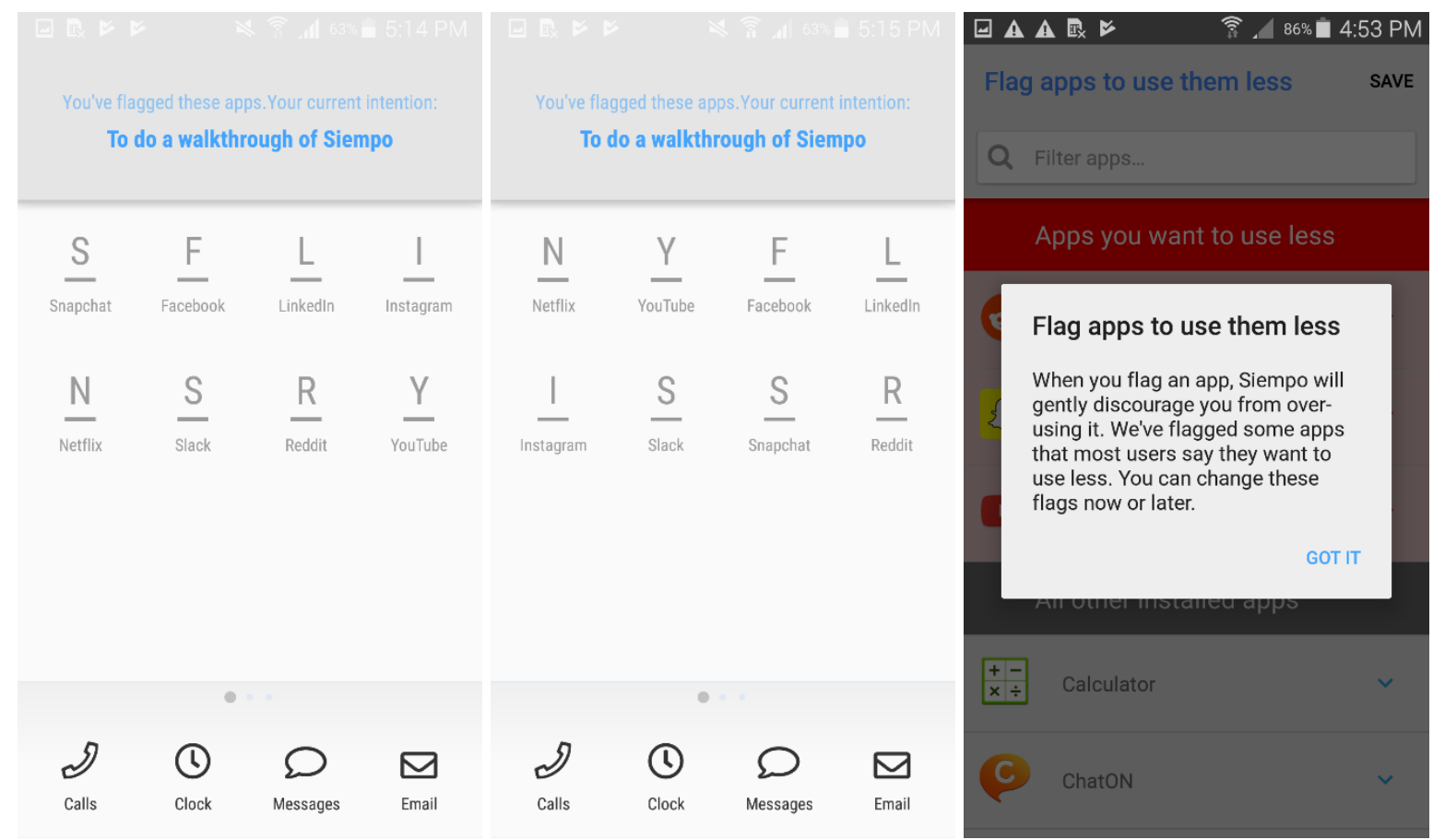

FIGURE 6-E: FLAGGED APPS OF SIEMPO

On the flagged apps screen app icons are muted, unbranded and coloured a faint grey. Apps are stripped of their branding and replaced by the first letter of the app name; Snapchat become "S" and YouTube becomes "Y". Underneath the letter is the name of the application. The location of flagged apps is also scrambled, meaning the position of each app is randomised to prevent unconscious selection and usage. Every time the user returns to the screen that houses flagged apps, the position of each app changes. Users are also given an opportunity to flag any apps Siempo did not do for them. When flagging an app, users are given an option to either 'flag this app to use it less', 'get info or uninstall app'. 


\subsubsection{Batching notifications}

A third feature of Siempo is that it batches notifications at temporal intervals. Users can choose when they wish to receive notifications via the bell icon on the top right-hand side of the home screen (Figure 6-F). When selecting the bell icon, the user is taken to an additional screen where they asked when they want to receive notifications. There are three options to batch notifications: as soon as they arrive (the default option on most phones); at customisable blocks of time; or only at a certain period of the day. To achieve either the second or third options, Siempo uses the Do Not Disturb functionality on Android devices to silence notifications until the user had indicated when they want to receive them.

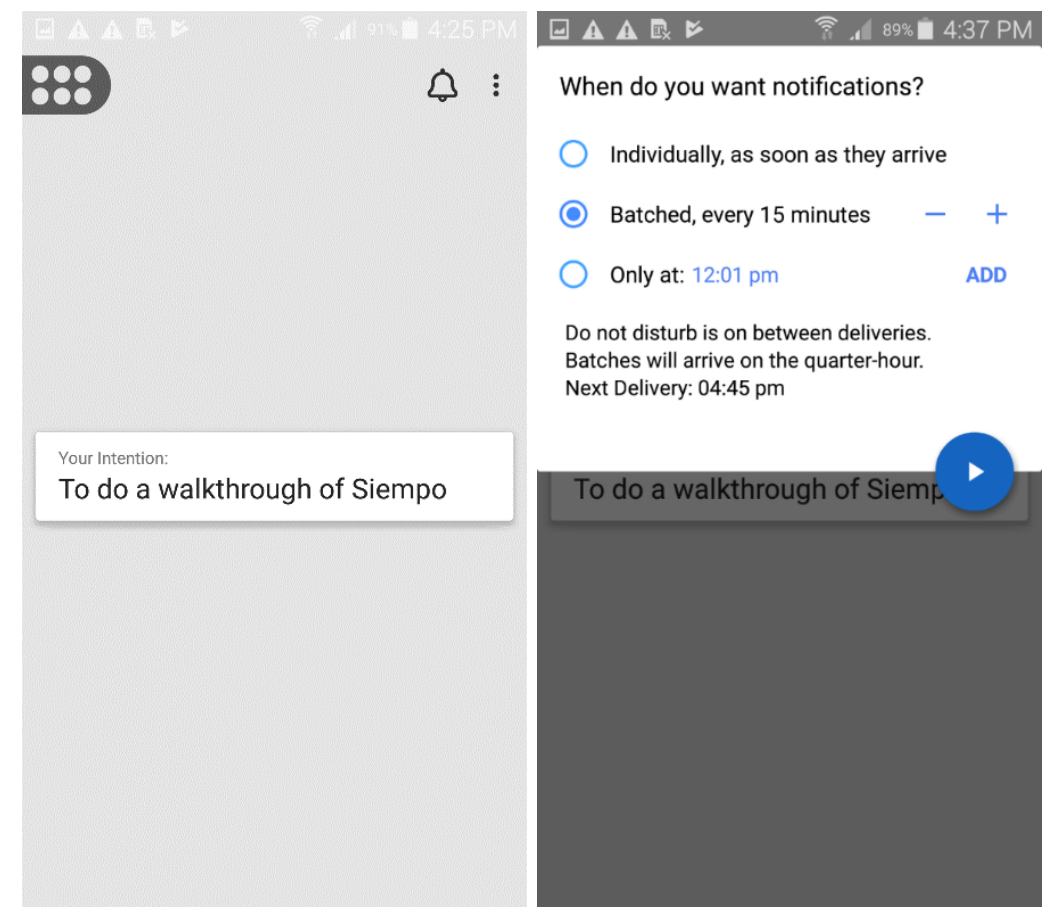

FIGURE 6-F: THE NOTIFICATION TEMPO FUNCTIONALITY

By delivering notifications on a schedule of the users' choice, Siempo embeds tactics of delay to intermediate a slower media experience. Users are not fully disconnected from the internet, but more dropped out from the subjective uncertainty that is set by the irregular 
arrival of communications via notifications. From an informatics or information engineering perspective, a delay on the standard notification delivery process is an inefficiency but, from a Silicon Valley dropout perspective, the delay offers greater autonomy to perform uninterrupted intentional activities. As users can determine when they are interrupted, the batched notifications feature offers a micro-temporality or an alternative schedule for the user. Being able to stagger notifications also directly relates to the name Siempo; as Dunn explains to me: "Siempo refers to always being mindful of your time and it also invokes simple and tempo, living your life at your own tempo."

The practice of delaying notifications also results in a more modest digital experience. Susanna Paasonen (2016) argues the difference between an unwelcome and a welcome digital distraction is impossible to categorise and depends on the user's subjective position. As Siempo bundles all notifications into the category of distractions, what batched notifications deliver is not just a slower media experience, but a dampening of what Paasonen (2018) calls 'affective intensities', the wide range of emotive feelings users commonly experience when connected to the internet. Affective intensities include the elation of receiving a congratulatory email, the boredom of scrolling social media, or the shame associated with receiving little to no social media responses. By temporarily depriving the opportunity for affective connection, batching notifications is akin to practicing asceticism, i.e., a momentary opt-out of the rollercoaster of online emotions in favour of periods of concentration and purpose.

Ascetism, or the deliberate abstinence from pleasure, is often something an individual imposes on themselves in the interest of self-improvement (Portwood-Stacer 2012b). Delaying notifications is therefore a reminder of Foucault's $(1997,282)$ interpretation of 
asceticism as, "an exercise of the self on the self by which one attempts to develop and transform oneself, and to attain to a certain mode of being." As I have argued in this chapter, a lauded mode of being in the Silicon Valley is intentionality, and Siempo produces an intentional subject position for the user to manage themselves as an autonomous and selfregulated but, critically, productive agent. Unconscious usages of the smartphone are implied as unhealthy or inappropriate for an increasingly time-precious society. To this end, the Siempo regime to self-govern behaviour draws similarities to the latest means of efficiency engineering (Gregg 2018) or work management and/or productivity tools (Guyard and Kaun 2018). By emphasising focus, Siempo also extends the capitalist vision of time, seeing all time in the day as needing to be maximised and productive (Gregg 2018). Siempo promises to unlock users' full potential and master the art of stress-free productivity equating a productive and happy life together. Apps like Siempo reflect a wider underlying cultural expectation of continuous improvement (Gregg 2018; Wajcman 2019), as if increased awareness (or the colonisation of everyday life) of one's behavioural data will necessarily result in a more rational apportioning of one's time. Moreover, as a consumer product, Siempo places users in their own micro-temporalities, individualising their experience of time.

\subsubsection{Screen cover}

A fourth feature of Siempo is a screen cover that punishes users who spend too much time on flagged apps. Screen cover can be turned on under settings (available to be selected as a sidebar on the home screen, see Figure 6-A). Users can set temporal limits on flagged apps from anywhere between one to twenty minutes. Once the setting has been switched on, if a flagged app is used beyond the promised time period, a screen cover appears from both the header and footer of the screen (Figure 6-G). 

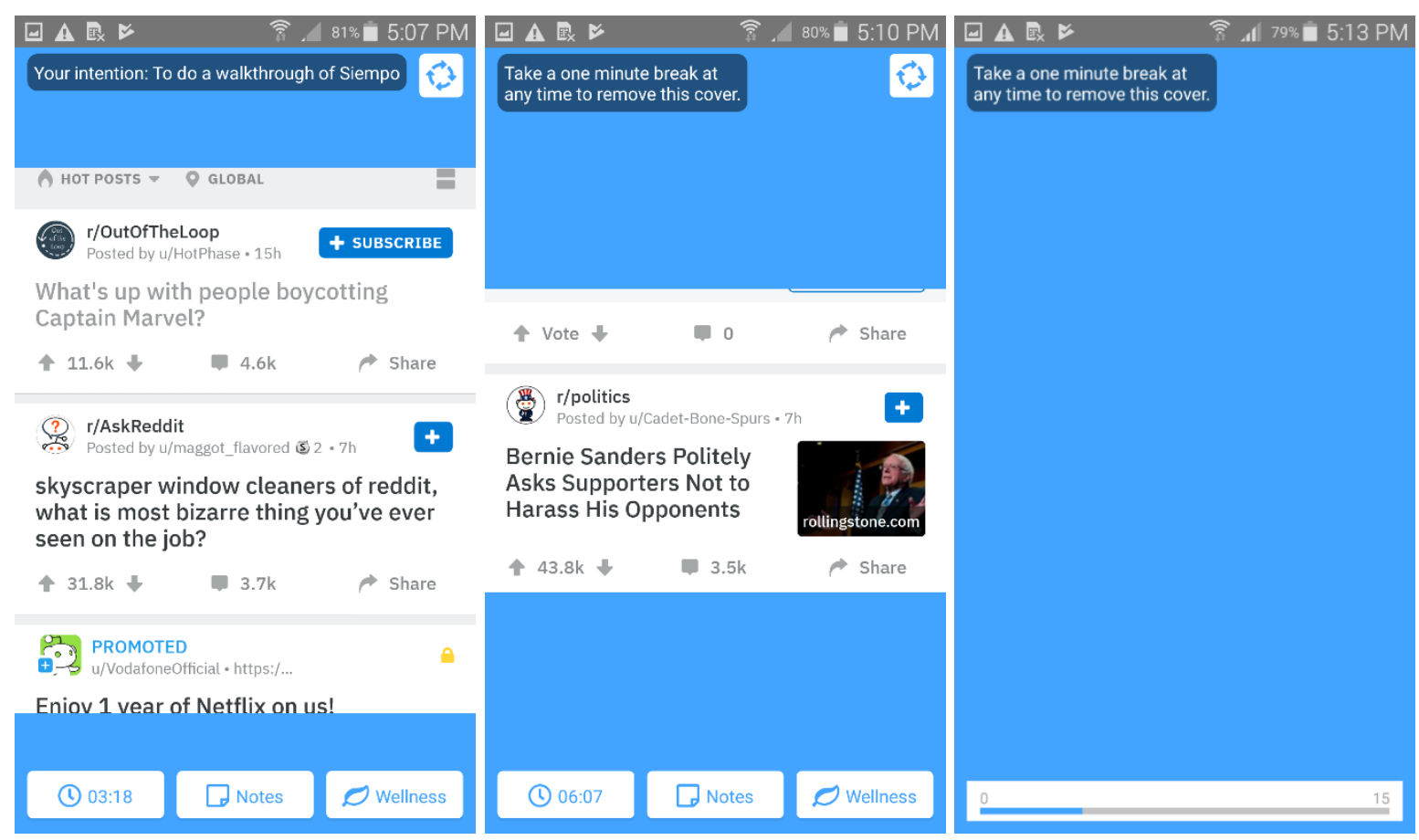

FIGURE 6-G: THE SIEMPO COVER SCREEN FUNCTIONALITY AT THREE, SIX, AND TEN-MINUTE INTERVALS

The screen cover is a persuasive technology design feature that punishes overuse of flagged apps. Messages and functionalities appear at the header and footer of the screen cover to discourage any further use of the flagged app. In the header, messages appear at one-minute intervals and rotate between reminding the user of their set intention, and to "take a one minute break at any time to remove this cover." Screen cover also acts as a motivational coach, with additional messages including: "Let your intention be your guide. We believe in you!", or "reflect on how this app makes you feel". Dunn reveals to me that Siempo combines a persuasive design with the cultural scaffolding of intentionality:

[Screen cover] takes a behavioural design approach [that] really manipulates the triggers and ability and motivation to create a digital environment that would align with your intentions.

At the footer of the screen is information of time spent on the app so far, as well as shortcuts rotating between "settings", "notes" or "wellness". Access to the notes and wellness apps during the middle of a behavioural intervention suggests that Siempo prioritises introspective 
activities. Siempo enables the user to annotate their feelings in the middle of a behavioural intervention and/or quickly respond to a distraction by undertaking a wellness activity such as meditation. The shortcut to settings is provided to enable the user to turn the screen cover functionality off. This flexibility dilutes the impact of the behavioural intervention as Siempo provide an easy means to escape punishment. If the user reopens the flagged app before a one-minute break, the entire screen is covered.

\subsection{The authentic digital consumer}

In a video produced by Siempo (2019) for potential investors, Andrew Dunn invites viewers to take deep breaths and reflect about the anxiety that can come from "mindless consumption" and technology use. Dunn describes Siempo as an intermediary technology that is "on your side", assisting users to make intentional technology and consumerist choices (Siempo 2019). The video is a reminder that Silicon Valley's drop-out ethos lives on in Siempo. Dunn does not deem consumption per se as problematic; rather, it is conventional consumption, which in the digital milieu means mindless scrolling, which is the problem. The Siempo video suggests that the goal of the Siempo app is to convince the user that intentional smartphone use is authentic smartphone use. Similar to how the Burning Man festival packages counterculture as consumable to new audiences, Siempoturns intentional living into consumable commodity that users can install on their technology devices to undertake authentic smartphone consumption. What is 'authentic' smartphone consumption is of course, subjective, but what Siempo does is broaden the scope for digital consumerism to capture new types of user authenticity. Excessive 'prosumption' (Ritzer and Jurgenson 2010) or 'produsage' (Bruns 2008) (terms to denote that users of the internet and social media both produce and consume content), is no longer considered an authentic digital 
practice by technologists like Dunn. In digital subcultures such as slow media, where users of media actively slow down their media consumption (Rauch 2018) and popular discourses about smartphone addiction (Turkle 2013; Twenge 2017) excessive time online or produsage is considered problematic and an inauthentic representation of the self (Jurgenson 2013). Siempo offers a means to disconnect from these 'inauthentic' modes of self-representation while remaining online. The authentic digital consumer constructed by Siempo constantly self-scrutinises what they click on, what comments they leave, and the images or videos their share, and so on. According to Siempo, to achieve online authenticity is to act with intention.

By providing an alternative practice of consumption, Siempo and the intentional self reinforce disconnection as a type of neoliberal lifestyle. Yet this is perhaps not the neoliberal lifestyle that Foucault envisaged as a mode to be less governed and resist being shaped by power. Users of Siempo do more than just intervene in the subject-making process forces produced by the attention economy. In addition to resisting spending too much time on their phone, users subjugate themselves to coercive regimes of power in relation to productivity and health. The jurisdiction of the moral may not be administered by the state, but instead derives from the pseudo-scientific Silicon Valley counterculture of intentional living. Moreover, inhabiting the intentional self subject position places the impetus of change on the individual instead of industry and political elites to provide less invasive technology design practices. The user is encouraged to better manage their own time while the overriding imperative of persuasive design to tether users to their devices is more or less unquestioned. Such intention-enabling features follow Siempo in disconnecting users from distractions but connect them to new regimes of consumerist practices that are positioned as key mechanisms of change. It may be that to achieve significant reform of the attention 
economy, scrutiny should shift away from the intentions of the user, to the intentions of those who engineer user behaviour.

In this chapter I have argued that the intentional self is a subject position embedded in the architecture of the app Siempo. I traced the history of the intentional self to a Silicon Valley 1960s and 70s counterculture that dropped out from mainstream society to innovate with technologies and emphasise personal transformation and goal-orientated living. Siempo channels the ethos of dropout counterculture into an intentional interface that intermediates the relationship between the user and their unconscious digital habits. Alongside an alternative notification delivery schedule, users can opt into an intentionally slower digital existence, primed for self-discovery and personal transformation. Siempo and the intentional self stand in contrast to conventional digital consumerist behaviours such as habitual use, offering a new and authentic type of lifestyle or consumption practice as a means to resist the attention economy. Yet the intentional self locates resistance to the attention economy at the level of digital consumption and places the impetus on the user to change their technology habits. Furthermore, the intentional self requires a degree of regular selfsurveillance of day-to-day activities that squeezes out spontaneous modes of living. In the final analysis chapter, I identify and analyse a type of technologised disconnection which requires a sacrifice that is arguably even more problematic. 


\section{The available self and the gendered inequities of disconnection}

After three years of being Facebook-free, I began to notice some of the consequences of being disconnected from social media. I could no longer remember the dates of friends' and family members' birthdays or put names to the faces of casual acquaintances. Given my time on Facebook had expanded my social circle well beyond the 120 people that anthropologist Robin Dunbar (1992) famously suggested as a limit to maintain stable social relationships, I decided that it was acceptable that I could no longer retain impersonal information about these so-called 'friends'. Without Facebook, I was no longer reminded by the social media platform to type an effortless 'happy birthday' on someone's profile, and neither were Facebook friends' names being reinforced to me as I did not have a news feed to scroll. However, really, I did not know anyone's birthdays pre-Facebook either. I have not had to manage my social calendar for years; instead, I have, admittedly, relied on the social and organisational savviness of my partner, who is a woman. Though anecdotal, this shows how my ability to disconnect-from Facebook, birthdays, or details about other people's lives-is intertwined with gender, agency and power.

In this final analysis chapter, I interrogate these tensions between gender, agency, and disconnection. I explore who can disconnect from the internet or attention economy more easily than others. To do so, I apply a gendered lens to disconnection. In the first section of this chapter, I foreground male subjectivity in relation to disconnection to demonstrate how problematic and inequitable dominant modes of disconnection are. I argue that the ideal disconnected subject is the Man Alone-a masculine figure who is unencumbered or connectionless, free from relationships, responsibility and the expectations that come with 
social ties. If left unchallenged, unencumbered disconnection becomes ideological, the common sense or 'authentic' mode of disconnection. Disconnecting is also an opportunity to exhibit masculinity and power. In response to unencumbered disconnection, in the second section, I analyse an app called Lilspace and a self-relation that it produces: the available self. The available self is based on poststructuralist feminist conceptualisation of an 'ethical' technology of the self, where the needs of others are foregrounded (McNay 1992, 2000). I argue that the available self is encouraged to disconnect in awareness of and in relation to other subjects and their dependencies. The available self does not sever social ties but suspends them. I close the chapter by critiquing the available self for reinforcing postfeminism and a fixed conception of the female subject.

\subsection{Ideal disconnected subject}

Some people are in less of a position to disconnect from social media, the internet or the attention economy than others. To explain why, I discuss the ideal disconnected subject and the discourses of hegemonic masculinity that produces this subject position.

\subsubsection{Hegemonic masculinity and the Man Alone}

The technology of power that produces the ideal disconnected subject is hegemonic masculinity. Hegemonic masculinity is a belief system and practice that legitimises men's dominant position in society and the subordination of women (Connell 1987, 1995). The concept was first conceived in a study in Australian high schools (Kessler et al. 1982) and was developed alongside Raewyn Connell's conceptualisation of gender as a product of hierarchical social structures. Hegemonic masculinity is defined by Connell and Messerschmidt $(2005,831)$ as a belief system that presents "the currently most honored way of being a man, requir[ing] all other men to position themselves in relation to it... ideologically 
legitimat[imising] the global subordination of women to men". The concept has been criticised for essentialising masculinity, reifying gender roles and not emphasising the historicity of gender (for an overview, see Connell and Messerschmidt 2005). Hegemonic masculinity has since been reconfigured as a sociocultural, multifaceted force that shapes and constrains men and women's agency, but is not wholly determinative of it (Messerschmidt 2018).

From a Foucauldian perspective that views power as materialising in discourses, hegemonic masculinity is a technology of power that presents a version of masculinity to which individuals subject themselves, and generates gender-orientated standards and norms to guide conduct between people (Nixon 1997). An example of a behaviour that has been discursively produced by hegemonic masculinity is 'locker-room talk', whereby men share crude (and embellished) stories of sexual escapades and label perceived feminine actions with derogatory homophobic slurs out of fear that any respect or tolerance towards femininity might be perceived as weakness. Artist and activist Grayson Perry (2017) describes the social pressure to perform hegemonic masculinity with a metaphor he calls the Department of Masculinity:

Somewhere in every man's head there is a governor, an unconscious inner voice sending instructions through the intercom. This governor is the boss of every man's personal branch of the Department of Masculinity. This Department wants to maintain standards. Every man's personal governor has picked up instructions from a variety of sources - parents, teachers, friends, films, TV, books - on what it is to be masculine. He takes ideas and images from these sources and assembles them into a model of a perfect man. The governor then sits there, constantly checking that his man is living up to this ideal. If the man fails, he is made to feel unworthy, he may hate himself, he may take it out on others. A man may not be aware of this governor - he may think that he is his governor or that men are 
free to behave how they like - but until he is aware of and understands the Department of Masculinity, he will be totally under its command.

The pressure of hegemonic masculinity encourages a regular independence or social disconnection from other people. Hegemonic masculinity confers a model of relations for how men should and should not relate to other people, and on what terms, contributing towards a process of male gender socialisation (Watson-Phillips 2016). Paul Gough (2018, 40) argues that men have been conventionally encouraged to act independently when processing emotions: "traditional masculinity norms have promoted rationality, self-reliance, and stoicism, with the realm of emotion disbarred, delegated to women, and coded as weakness or irrationality-except for specific emotions such as anger which are considered more 'masculine'." Hegemonic masculinity instead encourages an independent subject position that downplays the rewards of certain relational acts. Men, of course, experience emotions, but they are not traditionally encouraged to seek help or manage emotions interpersonally or relationally (Addis and Mahalik 2003), preferring to undertake (often limited) self-management strategies (Oliffe et al. 2010) that can allegedly contribute to suicide rates in young men (Barker 2005). It is not that hegemonic masculinity makes relational acts impossible for male subjects, but that cherished masculine norms such as self-reliance prompt men to police and internalise certain emotions that could be viewed as a weakness if shared with other men. Moreover, the fear of appearing weak in front of other men dictates that some men may only feel comfortable demonstrating vulnerability with women who, in the paradigm of traditional gender relations, become care workers. It is, however, important to emphasise that the independent male subject position is not fixed. For example, Carol Watson-Phillips (2016) contends fatherhood can liberate prior notions of manhood and masculinity, opening men to more regular caring and relational activities with their children. 
Numerous representations in Western culture have positioned the independent man as the ideal disconnected subject. In my country of Aotearoa New Zealand, the disconnected subject has historical relevance for Pākehā. A solitary figure is the title of the famous New Zealand novel Man Alone ([1939] 2010) by John Mulgan. The Man Alone ${ }^{1}$ is an ex-soldier called Johnson who arrives in New Zealand during the Great Depression to start a new life. The Man Alone is "the solitary, rootless nonconformist, who in a variety of forms crops up persistently in New Zealand writing" (McCormick 1959, 130), including Shiner Slattery(1964) by John A. Lee and lan Dougherty's Arawata Bill(1996). These novels position the Man Alone as an archetype of a typical New Zealand and Australian male with little to no social ties, who prefers to live by their own means. Gilkison and Shep $(2012,105)$ argue the Man Alone is a "paradoxical figure of both existential alienation and self-sufficiency," and "has perpetuated the iconic Kiwi myth of masculine identity founded on isolation, solitude and rugged individualism". The North American equivalent of The Man Alone may be 'American Cool', "a distinctly American twentieth-century emotional code of restraint and disengagement" (Shamir and Travis 2002, 1-2) with links to the Ernest Hemingway Code Hero² and celebrated aloof characters like the Lone Ranger. The Lone Ranger suggests a heroic element to the Man Alone, yet it is important to note that the subject position is largely not celebrated in literature.

Mulgan ([1939] 2010) problematises the original Man Alone as a figure living in latecolonial economic precarity suffering from cultural displacement and post-traumatic stress

\footnotetext{
${ }^{1}$ Author John Mulgan claimed he found inspiration for the Man Alone in the Ernest Hemingway novel To Have and Have Not, which in turn was influenced by John Donne's (1988) $17^{\text {th }}$ century poem No Man Is An Island.

2 The Hemingway Code Hero is a common character in Hemingway novels that is defined by a static set of characteristics: always courageous, confident, and silent. He does not let his fears get to him or show his emotions (Plath 1999).
} 
disorder. Johnson is a loner who struggles to integrate into New Zealand society with limited work and social opportunities, who is "lost or mired in a psychological minefield that causes them to withdraw into their shell" (Allen 2011, 89). The male and female relations the Man Alone has are constrained by his masculinity (Stachurski 2009), with the Man Alone disposition seen as a symbol of early $20^{\text {th }}$ century existentialism when the search for meaning and responsibility was largely understood as a solitary, individualistic exercise (Benson 1999).

Yet, the status of the Man Alone remains an aspirational position for many men. Part of the appeal of inhabiting the Man Alone is the possibility to escape and have a harmonious relationship with nature. In other words, it is not solely the underlying existentialism-or selfdefining choices - that makes the Man Alone appealing, but his transcendentalism ${ }^{3}$, meaning the seeking of sublime experiences that lie beyond human knowledge (Myerson 2000). A famous transcendentalist who retreated from civilisation to a cabin in Walden Pond in Concord, Massachusetts was Henry David Thoreau. The type of subjectivity that Thoreau championed was self-sufficiency and rugged individualism (Walls 1993). Feminist and literary scholars debate whether Thoreau's lifestyle is empowering to women, the answer to which depends on whether the female subject is expected to perform domestic labour and can inhabit the Man Alone subject position entirely by herself and not in addition to their gendered expectations (Goldman 1990; Walls 1993). Part of the appeal of Thoreau's Man Alone position is the escape from the domesticity of day-to-day life instead of being responsible for household work in a different setting. It is perhaps no coincidence that the majority of

\footnotetext{
${ }^{3}$ Transcendentalism has links to modern Silicon Valley culture, or New Communalism, that I discussed in chapter six. According to Turner (2006) Buckminster Fuller, the inventor of the geodesic dome, an iconic New Communalist technology, has a Transcendental lineage and was influenced by his greataunt Margaret Fuller, who was a well-known Transcendentalist. Margaret Fuller co-founded the Dial, the preeminent literary journal of American Transcendentalism with Ralph Waldo Emerson.
} 
subjects in "my year alone in the wilderness" videos on YouTube are men, suggesting the Man Alone is a particularly masculine fantasy, which (Western) women have not been socially conditioned to expect or desire.

The modern-day Thoreau and Man Alone is Christopher McCandless, whose story was captured by Jon Krakauer's (1997) book Into the Wild. Krakauer details the late 1980s McCandless's rejection of material consumption and conventional life to retreat to Alaska and "live a life untouched by the trappings of culture and civilisation" (qtd. in Power 2012). McCandless undertook a type of unencumbered disconnection that has been romanticised, despite McCandless dying from starvation after eating berries that complicated his digestive system (Krakauer 1997). Into the Wild sold more than two million copies, and a number of people (mostly men) have attempted to replicate McCandless' journey and visit his site of death, leading to hiking-related deaths (Power 2012). When asked what attracted him to turning Into the Wildinto a film, director Sean Penn answered it was McCandless' "decision to go back to the world" (qtd. in Power 2012), suggesting the Man Alone is an archetype of human behaviour that predates modern society.

Some entrepreneurs believe the Man Alone subject position is an optimal means to enhance productivity and mental fortitude. Silicon Valley entrepreneur Tim Ferriss calls for professionals to seek independence and solitude every day to build emotional resilience. Ferriss has been labelled a "tech-bro", a stereotypical white-collar software developer, engineer or entrepreneur that does not appear on the surface to be hyper-masculine but retains certain features of hegemonic masculinity (Chang 2018). The hegemonic masculine values that tech-bros such as Ferriss reintroduce are stoicism, which champions hard logic and the active subduing of emotions for personal gain. When discussing his view on relationships, Ferriss $(2011,82)$ writes: 


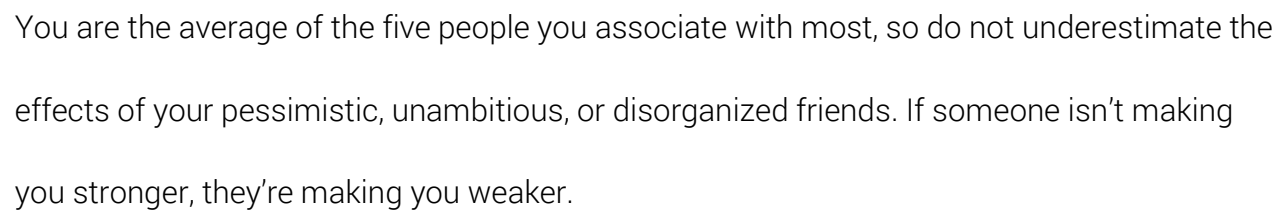

Ferriss is a Man Alone who advocates for severing unproductive relationships instead of, say, repairing them. Through the practice of tech-stoicism, Ferriss turns the Man Alone into an appealing and marketable subject position that can enable workers to acquire the edge to get ahead in their career. Watson-Phillips $(2016,285)$ contends that men influenced by "stoicism learn to suppress feelings, harden their ego boundaries, posture as invincible, disconnect from others, and lose their genuine sense of being-all behaviours that are counter to building and maintaining relationship[s]." By offering a narrow view of the benefits of relationships, Ferriss' stoicism encourages emotional and relational immaturity.

From these examples, we see that the Man Alone subject position and hegemonic masculinity reinforce each other. The Man Alone is the ideal disconnected subject because the Man Alone is an independent and unencumbered figure who is free to disconnect at any time to make autonomous decisions or move closer to nature or work. He has ample social mobility, not in terms of class mobility but the agency to cut social ties at whim. Conversely, to disconnect on the terms prescribed by the Man Alone requires a disregard for social relations, a key tenet of hegemonic masculinity. To disconnect under the gaze of hegemonic masculinity comes at a cost that a wide range of people are not able, nor willing, to take. In the next section I discuss these categories of people.

\subsubsection{The affective labour of managing connections}

One problem with the ideal disconnected subject position is that it is not available to everyone. To sever social ties at whim without significant consequence requires a type of agency that can transcend relationships. In poststructuralist theory and feminist studies, 
agency is understood as a relational process shaped by a variety of constraints, norms and expectations (Gill 2007). From a poststructuralist perspective, women have less agency to disconnect because they are expected to undertake relationship-orientated tasks that preclude them from doing so. There is the broad responsibility of managing connections that includes tasks such as replying to emails or texts, sending happy birthday messages on social media or simply checking in on close friends and family. These tasks are generally undervalued because they are not what men are conventionally expected to undertake. Portwood-Stacer (2014) compares managing social connections on social media to care work:

Overall, the labour of online social networking bears a striking resemblance to what has traditionally been constructed as 'women's work'-the caring and relational labor involved in holding communities together and reproducing the conditions that support members' everyday survival, wellbeing, and capacity to contribute to society. ${ }^{4}$

Managing connections requires affective labour, where generating a "sense of connectedness or community" (Hardt 1999, 96) becomes work. To highlight affective labour is to recognise that women are burdened with invisible obligations and responsibilities that preclude them from disconnecting. Portwood-Stacer (2014) goes on to argue that men benefit from the care work undertaken by women in their lives:

\footnotetext{
In my observation, Facebook mirrors the offline social world, in which women plan the gettogethers, send the birthday and holiday greetings, transmit the family gossip, and just generally stay present in everyone else's lives. The men of course benefit from all this-they attend the family gatherings and certainly keep abreast of the news that gets announced online-and this is made possible by the time and effort their partners put into social
}

\footnotetext{
${ }^{4}$ There is a rich body of scholarship that ties the labour women have traditionally provided as caregivers to online immaterial labour (Dyer-Witherford 1999; Huws 2019).
} 
networking. It's work the women are genuinely happy to do; they are sincerely devoted to their families and they are comfortable in a social role in which they facilitate the transmission of information, affection, and resources among their loved ones.

Portwood-Stacer's contention that women are "genuinely happy" to manage online connections could be interpreted as controversial. My interpretation is that Portwood-Stacer does not mean to naturalise social media work as a women's job, but is instead acknowledging that the maintenance of social connections can often be legitimately enjoyable. In addition, social media understood as care work refutes the popular view that social media is a predominantly narcissistic endeavour (see Twenge 2017) or a platform where users are 'addicted by design' (Harris 2016), positions that have been discussed on a number occasions throughout this thesis. Instead, a perspective on mediated affective practice recasts social media as a place that requires emotional and affective labour from its users as real affective ties are forged and maintained as social capital (Ellison, Steinfield, and Lampe 2007). It is these relational and affective dimensions to social media that can make disconnection difficult, as meaningful bonds are generated and managed by social media platforms (Carlquist, Prøitz, and Roen 2019; Karppi 2018). If elements of subjectivity are circulated throughout social media and networks (Giraud 2015) such as the users' capacity to be affected, care and feel a sense of belonging for others, it is not surprising that some users may not want to disconnect at all. The affective dimensions of managing connections are arguably even more pertinent when affects and a sense of belonging are amplified by platforms that are powered by algorithmic circuits (Wark 2019) and political economies (Dean 2005) beyond the user's purview.

The Man Alone who effortlessly disconnects from social media is not obligated to subject himself to such regimes. Free from the expectation of managing social life, the Man 
Alone has the agency to bypass the affective circuits of social media. More than that, hegemonic masculinity demands the Man Alone does not lower himself to the affective thrills of social media. In 2018, inventor and entrepreneur Elon Musk was asked by his Twitter followers to delete one of his company's (Space X) Facebook account after the Cambridge Analytica data scandal story broke. Musk was goaded by some followers to "be a man" and cut his ties with the social networking service. In a recounting of the interaction Grush (2018) writes: "A [Twitter] follower told [Elon] Musk he should delete Space X's Facebook page if Musk was 'the man'. Musk claimed he didn't even know it existed and then said he would, probably in an attempt to preserve his 'the man' status." That deleting a social media account was positioned as an opportunity to exhibit masculinity is a reminder of the relationship between hegemonic masculinity and disconnective behaviour. On one level, showing the ease with which one can cut their professional and social ties demonstrates agency and retains masculinity. Conversely, being seduced by the feminine affective bonds generated by social media is a sign of weakness and reduces masculinity.

There are other people who may not have the agency to disconnect from the internet so easily. For artists, contractors, or entrepreneurs, affective labour on social media is a professional necessity, in the form of undertaking actually remunerated labour, marketing their product or service, or obtaining future employment (Duffy and Hund 2015; Kuehn and Corrigan 2013). Journalism, public relations and academia are just a few examples of professions where workers are expected to self-promote their work. Moreover, any remote, flexible, or shift worker may depend on social media for the collegial support that they would otherwise receive from inhabiting traditional work environments (Gregg 2011). Social media also functions as a social security blanket for workers who want to remain flexible, available and likeable. For some professions, not having an active social media presence can hurt 
future employment opportunities (Grasz 2015) due to the self-branding opportunities of social media that education, entertainment sectors, among others, expect from employees and freelancers (Duffy 2015; Hearn 2008; Marwick 2015). Not everyone has the agency to leave Facebook because their (potential future) personal and professional responsibilities are wrapped up in the social media platform. Most recently, the \#deleteFacebook campaign that emerged after the Cambridge Analytica data scandal was criticised for neglecting to acknowledge the affective and professional cost of leaving the social media platform (Glaser 2018; Mitesser 2018).

Portwood-Stacer (2014) turns to the autonomist feminist movement to enable women to refuse social media and care work. Autonomist feminism was an Italian Marxist movement in the 1960s and 70s that resisted the positioning of domestic labour as women's work by emphasising that labour could be undertaken outside the factory (Federici 1995). According to Portwood-Stacer (2014), an autonomist feminist might see some instances of media refusal as 'engaged withdrawal' from an exploitative system of work (Virno 1996). The autonomist approach to labour recognises that domestic labour may be refused, rather than unthinkingly adopted as a natural state of affairs. As Weeks $(2011,124)$ explains, this was one aim of the autonomist feminists who demanded "wages for housework"; they wanted not to valorise domesticity, but to make it something women could feel empowered to refuse rather than destined to embody by virtue of their biology (Federici 1995). Managing connections and other forms of affective social media labour could similarly be refused by empowering the people who undertake invisible and unrecognised labour. However, my focus is not to argue for wages to manage connections, which relates more to immaterial labour or data rights, and sits outside the scope of this thesis. I instead turn to a smartphone 
application that attempts to empower female users to refuse social media by manufacturing a different way of disconnecting.

\subsection{The social disconnection of Lilspace}

To conceive of an alternative mode of disconnection I turn to the poststructuralist or postmodern feminist scholarship of Lois McNay. In the late 1980s and 90s, McNay (1992, 2000), identified a limitation to Foucault's writings on ethics and caring for the self. Her core contention was that Foucault's technology of the self assumes an unencumbered individual or autonomous self that comes at the expense of relations with other people. For example, Foucault $(1988,7)$ argued: "One must not have the care for others precede the care for self. The care for self takes moral precedence in the measure that the relationship to self takes ontological precedence." Despite Foucault's oeuvre dismantling dominant modes of thought and deconstructing Enlightenment subject/object dualism, McNay suggests that Foucault did not entirely escape a male knowledge production system. In particular, McNay $(1992,169)$ argues that Foucault's "vision of the unencumbered self is a masculine one because the degree of separateness and independence it postulates among individuals has never been the case for women." In other words, McNay considers Foucault's mode of resistance to presume a relatively isolated subject that can operate with a degree of relational mobility that has rarely been afforded to women. As discussed in the previous section, many of the social obligations or domestic labour that women are expected to undertake are relationshiporientated or obligated. Many women and workers have day-to-day burdens and responsibilities to undertake; moreover, some people may not want to shed their relations of nurturance (Driver 2017) or co-dependence (Weir 2013). For many, the desire to disconnect may clash with a wish or social expectation to be available for others. These scholars are not 
arguing that there is a presumed category of 'woman' that exists prior to caring practices, but instead, that caring practices have the effect of reinforcing the relational obligations and duties that women are expected to undertake.

As discussed in previous chapters, 'disconnection' can come in various forms, including more than just cutting social ties. A smartphone app called Lilspace resists the ideal disconnected subject by offering a type of disconnection for users who wish to remain available to others. I call this type of disconnection the 'available self'. Lilspace is available on the Android and iOS store and was developed by entrepreneur Kim Cavallo. Cavallo is also a community builder, which is a professional with a broad set of marketing, operations and business skillset who applies their skills to community-based activities (Burelle 2017). Cavallo implies that her intended user of Lilspace is a middle-class and middle-aged woman, particularly a mother, and that she believes there is not many technologies of disconnection that are aimed at this target market.

Relationships are at the core of the Lilspace ethos, with Cavallo telling me during our interview about the social impact that disconnecting from the internet can have on relationships. Identifying as a digital wellbeing company, Lilspace connects digital wellness to partaking in "real-life things" like physical exercise, community events or face-to-face conversation (Lilspace 2019). To Cavallo, digital wellness means having rich 'real life' or offline relationships, which reinforces the on/offline dualism. During the interview I conducted with Cavallo, she explained:

So, I could start off by saying I have a wellness practice and it seems natural to start a company or build a business around something I know. Obviously, the community building piece of it I know. That's not exclusively to women. But what I know from myself is the women in my life are very conscious of relationships to themselves and to others, and if 
we're in a group that's half men half women, in general the women are talking about their relationship with their kids or their relationship with their husbands, or there's just a lot more talk about their relationships, and I don't want to generalise but that happens normally. And the men are generally talking about their golf game and work and just a little less relational stuff.

For a relationship to remain 'healthy', it requires active work. Conversely, social disconnection prohibits a person from "participating in mutually responsive and mutually enhancing relationships" (Miller 2008, 372). To make disconnection relationship-friendly, Lilspace reconfigures disconnection as an extroverted, social and caring exercise. Lilspace users do not disconnect alone but do so with others who may wish to disconnect with them or have knowledge of the user's disconnection. In my walkthrough, I focus on two features of Lilspace: setting a status of disconnection, and disconnecting around common purposes or activities.

\subsubsection{Setting a status of disconnection}

Lilspace users are encouraged to nurture their relationships throughout the process of disconnecting. Before Lilspace users unplug, they are encouraged to notify their social networks (see Figure 7-A). This is made possible because Lilspace integrates with Facebook, Twitter and Slack application programming interfaces (APIs) to provide their users with the option of creating a status that integrates with their social networks. There are a variety of pre-set status updates available to users including: "At lunch, BRB.", "Hanging with family" and "Unplugged with lilspace." If the user wishes to, they can customise their own message via an open text field. The available self that Lilspace demands of its users anticipates and manages the communicative expectations of others. Setting a status of disconnection to manage the communicative expectations of others is similar to a shopkeeper who has communicated 
that they are taking lunch break but do not want to lose the opportunity for future business once they have return to the shop. Both are communicative strategies with the aim to temporarily suspend availability, while not severing it completely. Once the status has been set, the user can freely disconnect knowing that people who may be affected by their decision to disconnect are aware of what they are doing. By providing an option for users to communicate their disconnective behaviours, Lilspace allows for the available self to disconnect andmeet any the communicative obligations that they might have. Connections are managed despite the temporary social disconnection from them.

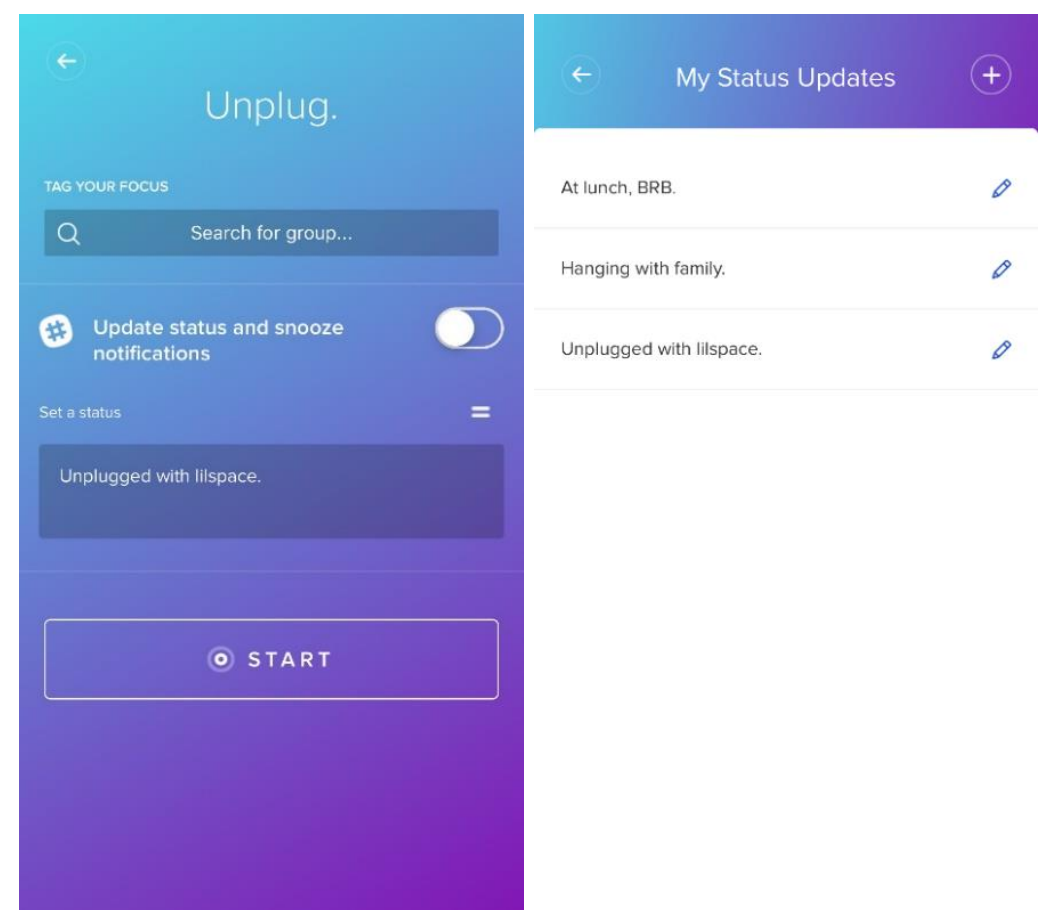

FIGURE 7-A: SETTING A STATUS OF DISCONNECTION

Lilspace acknowledges there are communicative and social risks to disconnecting, producing an available self that is sensitive to the needs of others. A user is provided with the opportunity to disconnect in a way that acknowledges that they value their relationships. To Lilspace, a 'healthy' disconnection is one where the needs of others are not compromised 
during the process of disconnecting. However, considering the needs of others does not stop at setting a status of disconnection.

\subsubsection{Disconnecting within circles of influence}

Lilspace also encourages users to disconnect around a shared purpose, activity or event. On the homepage users are provided with a list of activities, causes and groups to associate their practice of disconnection with (see Figure 7-B). There does not appear to be any clear difference between the categories of 'activities', 'causes', or 'groups' other than providing an opportunity for the user to associate their anticipated disconnective practice with something larger than themselves. Examples of an activity include 'Reading', a group such as 'Mindful Eating', or supporting a cause for an organisation such as The Rape Foundation. Clicking on an activity, cause or group takes the user to a distinct page where additional information is provided. On the page for the group Mindful Eating, users are notified that they will receive a reward of an e-guide of food recipes if they track one hour of disconnecting. Activity, causes and group pages also disclose statistics about the number of unplug sessions, hours unplugged, and how long the user has disconnected. 


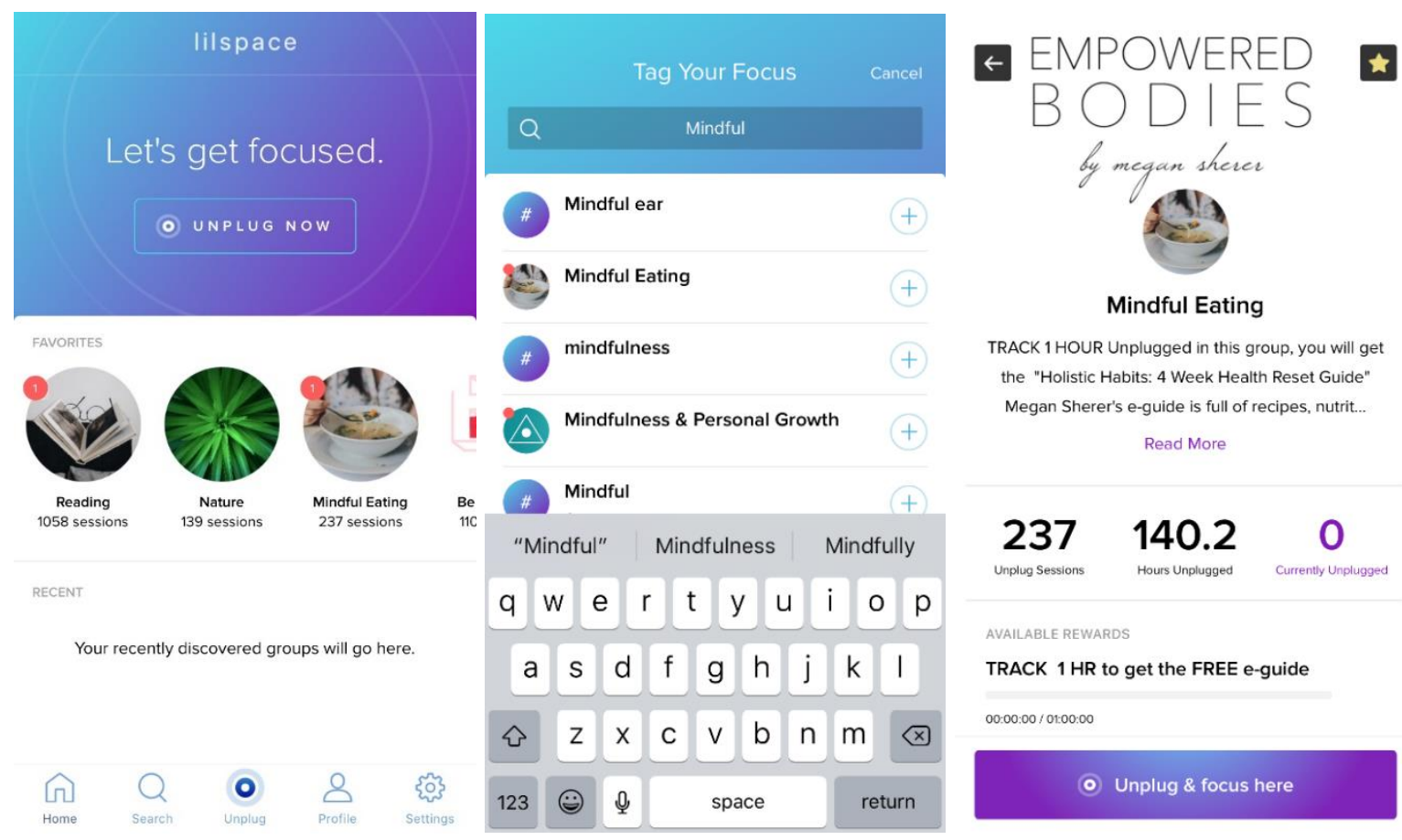

FIGURE 7-B: THE LILSPACE HOME SCREEN

By encouraging users to tag and share their disconnection, Lilspace makes the practice of disconnecting a potentially more affective or emotive practice. The option to disconnect for a shared activity generates 'affective bonds' (Karppi 2018) as expressions of intent and care are integrated into acts of disconnection. Disconnecting is imagined as a practice beyond the isolated individual as the act of disconnection can serve something greater and/or with somebody else. Users are encouraged to fashion themselves in a way that not only creates a more critical self-relation (Foucault 1988) but, in addition, a relation to others (McNay 1992). A Lilspace user might be inspired to disconnect from their phone because they wish to add to the collective pool of hours that they are informed others have disconnected for. In addition, disconnecting around a shared activity may influence relationally minded people to disconnect. Cavallo believes the best means to help people disconnect is through interactions with other people, or what she refers to as 'circles of influence': 
When you use Lilspace you see how many people are unplugged at the same time, and so again you're not alone, that kind of feeling. We want to expand that because people have given us feedback, such as, well the numbers [are] really cool, but I want to know if my mom has also unplugged at the same time, so [we give] people the opportunity to form these circles of influence.

A circle of influence is a concept that draws upon behavioural design frameworks that recognises the influence of peers on others (Stibe 2020) which provides an alternative model of relations for disconnecting. Disconnecting becomes a prosocial activity and less a form of social withdrawal. Such a model of relations conveys a different technology of the self than what Foucault (1988) envisioned. Foucault's (1988) technology of the self drew upon white privileged men in the Hellenic and Roman period that prioritised self-mastery with the goal to cultivate a self-aware sexual appetite. In contrast, Lilspace is less about abstinence and selfcontrol and more about coming to know oneself to achieve 'ethical' forms of freedom. The available self asks users to consider something other than themselves. To conceive of an other-orientated care of the self, McNay (1992) draws upon Jürgen Habermas' (1987) theory of communicative action, which proposed a way of understanding how the identity and actions of the individual can be mediated through interactions with others. Habermas (1987) saw communication activities as offering the potential to generate mutual understandings and achieve social integration and solidarity. On Lilspace, the other is an activity, cause or event, which are accorded a central role rather than a passive and subordinate one in the process of disconnection. Users are encouraged to disconnect for the purposes of an activity, cause or event. The available user of Lilspace does not just disconnect, but does so for a reason that might resonate with other people. The purpose of adding the 'other' to the process of disconnecting is to make disconnecting more appealing to people who prefer to socialise their activities or undertake them for reasons beyond themselves. Lilspace 
demonstrates how a more social variety of disconnection can emerge when relationships, activities or people are acknowledged and integrated into the process of disconnecting. The available self offers a distinct variety of disconnective practice compared to solitude or time spent alone, signifying a qualitative shift away from cutting social ties to considering which connections are worth cultivating and sustaining.

Although Lilspace integrates the idea of a circle of influence into the method of disconnecting, Lilspace itself is not a social networking platform. Users are not placed into a shared digital space once they choose to disconnect. One of the few social functionalities of Lilspace is the option for users to invite friends to try Lilspacevia an automated text message. Neither does Lilspace offer complex protocols to govern the disconnection. Once the user selects the 'starting unplugging' button, they are taken to a new screen and a time begins (Figure 7-C). There is no software protocol stopping the user from shutting down the app and using their phone for another reason. In addition, users are given the option to provide social media updates during the period of 'disconnection'. There is also a button to stop unplugging, which pauses the session. 


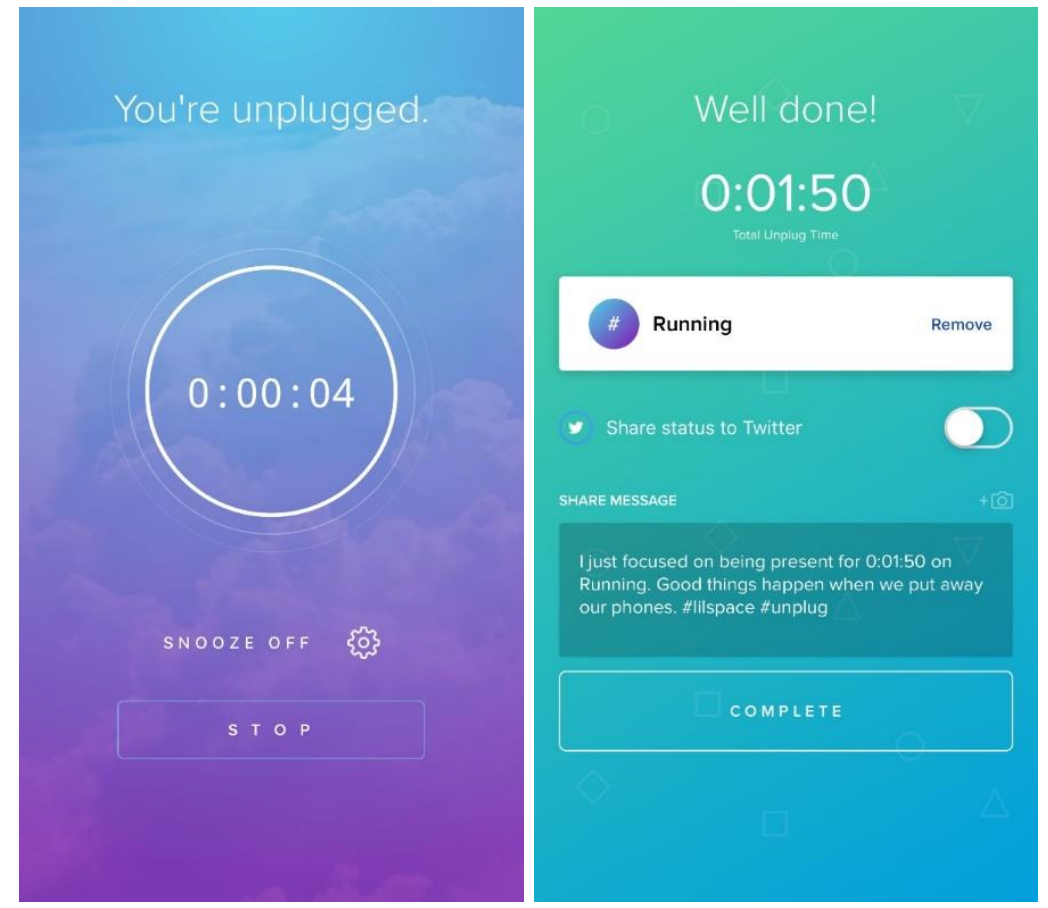

FIGURE 7-C: FURTHER STAGES OF THE LILSPACE UNPLUGGING PROCESS

If the user chooses to complete the session, they are rewarded with a new screen with the praise of 'well done!', information on the time the user disconnected for, and an opportunity to share their session over social media with a pre-set but customable tweet. Announcing the completion of a disconnection to social media is a prosocial activity that reinforces that disconnecting with Lilspace is not about acting in isolation but is a prosocial exercise to be undertaken within circles of influence. The intention of the functionality is to make disconnecting socially contagious. The logic of Lilspace is that if a friend in the network of the user learns of the disconnection, they might choose to disconnect, too.

\subsection{Ideal female disconnected subject}

Lilspace and the available self raises several gender-related issues. The available self does not provide an equal opportunity for users to disconnect from the internet, but instead manufactures a different type of disconnection that is intended for users that struggle to disconnect like the Man Alone subject position. In this closing section I critique the available 
self as a mode of resistance for reinforcing postfeminism and a fixed conception of the female subject.

\subsubsection{Disconnection as an ideology of choice}

Lilspace reconfigures disconnection through an ideology of choice rather than challenging unequal gender relations. The ideology of choice is a neoliberal set of values that merges emancipation with the freedom to make consumerist choices (Shun-hing 2009). In the case of Lilspace, users are offered a variety of incentives for disconnecting through consumerist choices (see Figure 7-D). Disconnecting through ample choice offers a new means for Lilspace users to understand themselves and the practice of disconnection. Cavallo believes the potential for choices while disconnecting in turn creates an opportunity for brands and business:

I wanted to find a way to help brands interact and engage with their customers, followers in way that would produce healthy behaviour. So encouraging, allowing brands to interact with their customers to encourage people to umm unplug and give them rewards for doing it. And the other piece of it-because I come from the non-profit world-is I also feel like one of the incentives is being able to help people in need or causes that you care about. So being able to have this bankable time, this idea that you have time you're accumulating by putting away your phone and being able to use that time to either do something healthy for yourself, because all the brands we interact with are wellness brands. So it's wellness products, events, or services that you would get discount codes for by unplugging or be able, and this part we're still figuring out, but right now Lilspace makes donations and that's going to run out quickly. So we partner with non-profit, or we partner with influencers who have nonprofits that they care about and then we let people unplug for that cause, and we're been donating a dollar for every hour. And so far the biggest donation we've made is about $\$ 850$ dollars. 
By emphasising choice and aligning the consumerist interests of users with brands, the gender politics of Lilspace is more postfeminist than feminist. Postfeminism is a liberal strand of feminism that emphasises personal choices for consumption rather than challenging gendered power relations and constraints (Banet-Weiser 2007). On Lilspace users disconnect to "discover new needs, new social positions and new relationships with nature, culture and society" (Shun-hing 2009, 51). Disconnecting with the help of Lilspace becomes a consumerist or charitable exercise. If the user wishes to, they can disconnect for a local event or a cause (see Figure 7-D). Each cause or event has a separate page to provide more information and the links to Lilspace. If a collective amount of unplugged time is reached, then a donation is made by a specified organisation. There are also discounts offered for specific places, museums, or restaurants. Disconnecting for charitable causes and events draws upon Cavallo's background as a non-profit community builder. For over twenty years, Cavallo produced fundraising events and campaigns in her community and saw Lilspaceas a way to channel user's attention to worthy causes. 

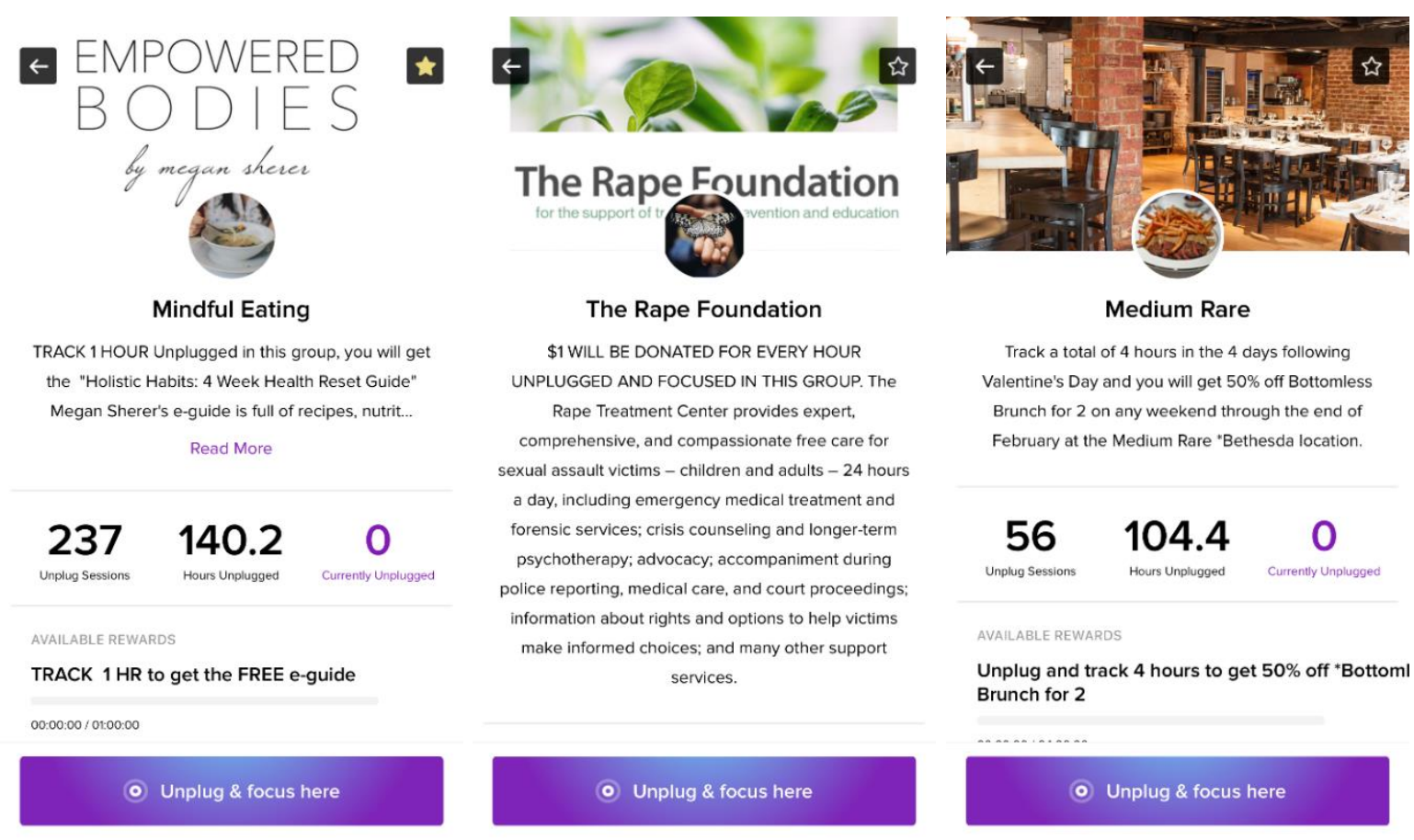
sexual assault victims - children and adults -24 hours a day, including emergency medical treatment and forensic services; crisis counseling and longer-term psychotherapy; advocacy; accompaniment during police reporting, medical care, and court proceedings; information about rights and options to help victims make informed choices; and many other support services.

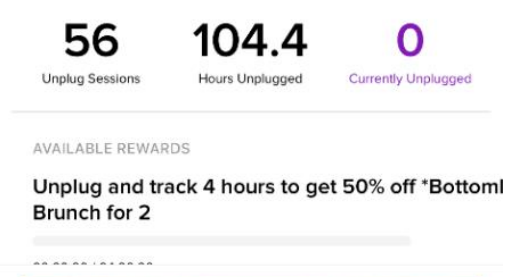

( Unplug \& focus here

○ Unplug \& focus here

\section{FIGURE 7-D: LILSPACE UNPLUGGING ACTIVITIES, CAUSES AND PLACES}

By encouraging users to disconnect for a cause, the available self produced by Lilspace is itself very available to co-option by branding and neoliberal self-enterprise. Ouellette and Arcy $(2015,101)$ suggest that neoliberal self-enterprise is at the core of Foucauldian ethics: "in the West today, the most pervasive and validated framework for practicing an ethical relationship to oneself is neoliberalism, which encourages the extension of market principles into all dimensions of social life, including subjectivity." Lilspace similarly merges market principles with the available self and extends market principles into the area of digital disconnection. Within the paradigm of neoliberalism, the type of disconnection that Lilspace offers is more about what social capital the user can generate. The available self is instructed to disconnect for specific reasons, turning the action of disconnection into a commodity, instrumentalised and put to use for activities and social causes. 


\subsubsection{Reifying the female subject}

The available self also reinforces gender norms instead of dissipating them. Instead of challenging the terms upon which women can disconnect, the available self is a fixed category of woman that offers a limited mode of disconnecting from the internet. Any fixed category of woman is produced by discourse (Butler 1999), or what I refer to in this thesis as a technology of power. Lilspace appears to draw upon discourses of hegemonic femininity, which are dominant ideals about women that reproduce hierarchical social relations (Schippers 2007). In the case of disconnecting from the internet, Lilspace produces expectations in relation to social obligations and communicative un/availability. A user of Lilspace might not know how they want to spend their time of disconnection other than knowing that they wish to disconnect. Yet Lilspace provides a specific and narrow idea of how the imagined female subject should disconnect. Users are encouraged to make their practice of disconnection social and notify their networks of their activities. The social requirements of Lilspace are comparable to Absher, the controversial Saudi 'wife-tracking' app that provides men location information about their wives (Bennett 2019) which reinforces problematic power dynamics. Lilspace is therefore an agent in anticipating the subject position of the user and shaping how the user should or should not disconnect (Mackenzie 2013). The user is encouraged to externalise their interiority (i.e. internal motivations to disconnect), for the benefit of a third party or the purposes of a cause or event. Such exteriorisation bears similarities to the techniques of confession, guidance and obedience that were crucial components of the effective functioning of pastoral power and placed the individual in an intimate relationship to the confessor (Foucault [1976] 1990). Lilspacemay enable users to disconnect and resist the attention economy, but it does so in a way that 
encourages the user to justify and share why they wish to disconnect, reinforcing the category of women as socially or relationally motivated.

The reality is that the Man Alone subject position is likely to be appealing to many users who identify as women. Lilspace could make the Man Alone position more available by making gender relations actionable to change. Lilspace could, for example, offer a functionality where women users in heterosexual relationships can request a (more privileged) male partner to become available on their behalf so they can disconnect as the Man Alone can. Women-only Facebook chat groups (see Pruchniewska 2019) are an example of a type of disconnection that attempts to rectify unequal gender relations. Private safe spaces are desirable for women and other minorities who cannot represent themselves fully through action or behaviours, in relationships, or be honest about all aspects of themselves (Russell 2009; Wheatley and Vatnoey 2020). The disconnection that derives from the creation of a safe space is not only about enhancing individual choice or autonomy but more a collective strategy for marginalised people to survive the harmful aspects of hyper-masculine or heteronormative culture together.

This chapter has critically assessed the tensions between gender and disconnecting from the internet. I first argued that hegemonic masculinity is a technology of power that produces the Man Alone: an unencumbered subject that can cut social ties at whim and disconnect largely without social or cultural reprieve. I suggested that the Man Alone is a subject position that is not available to many people-particularly women and workers who are socially expected to undertake the affective labour of managing online connections. Following this, I walked through the app Lilspace, demonstrating how it offers an alternative mode of disconnecting from the internet in light of these gender inequities. Calling this alternative mode of disconnecting the available self, and drawing upon feminist critiques of 
Foucauldian ethics, I pointed out how users of Lilspace are encouraged to disconnect in prosocial, extroverted and caring ways. I then criticised the available self for producing disconnection through an ideology of choice and a fixed idea of what the female disconnected subject is.

Now that I have identified and discussed all five relationships with the internet that the manufacture of disconnection produces, I summarise the viability of the manufacture of disconnection as a mode of resistance to the attention economy in my final chapter. 


\section{Part IV}

Conclusion 


\section{A technology-based culture of disconnection}

In 2018, Apple and Google both announced to the world that they were disconnectionists and were manufacturing their own technologies of disconnection. On 5 June, Apple (2018) released a new feature called Screen Time, a set of functionalities that were native to the Apple operating system (iOS) 12 to help users "better understand and manage their time on device". Barely a month later, Google (2018)—one of the leaders of the attention economy (Wu 2016)_announced a similar set of native tools to the Android operating system called Digital Wellbeing. Both Screen Time and Digital Wellbeing provide data analytics about time on device and enables the user (or the user's parents) to set limits on certain apps, as well as pause notifications that the user identifies as distracting. Apple and Google are not the only technology companies that have publicly announced a position opposing constant connectivity that integrates aspects of the disconnection movement into their ethos. In January 2018, Zuckerberg (2018) announced that Facebook would alter its newsfeed algorithm to ensure that time users spend on the platform is "time well spent". That phrase is, not coincidentally, the original name of prominent disconnectionist Tristan Harris' Center for Humane Technology. In 2017 Finnish telecommunication manufacturer HMD Global released a revamped model of the classic feature phone the Nokia 3310, dubbed the 'digital detox' phone (Sulleyman 2017). These examples suggest that both the tenets and products associated with the manufacture of disconnection have been embraced by the technology industry.

The interest by major technology companies in disconnection presents an opportunity to reassess the manufacture of disconnection as a mode of resistance to the attention economy. From one perspective, the manufacture of disconnection appears to have 
effectively enacted change in the attention economy as technology platforms such as Google and Apple have adopted similar technological practices. Conversely, it could be argued that the manufacture of disconnection offers a type of resistance that is non-threatening or even appealing to technology platforms. Should we conclude that the manufacture of disconnection meekly resisted the attention economy and has been integrated into the platform?

Not quite. In this closing chapter, I summarise the key findings of my thesis and show how the manufacture of disconnection resists some aspects of the attention economy while transforming other technological practices within the attention economy. To make this distinction explicit, I summarise the self- and space-based relations that are produced by the manufacture of disconnection and demonstrate how these architectures constitute a wider technology-based culture of disconnection. I argue that the technology-based culture of disconnection is embraced by technology platforms when technologised disconnection presents new tools for users to better control their behaviours in mediated environments. The manufacture of disconnection is not only about resistance, but also enhanced governance of the self, other users and technological practices the Silicon Valley disconnectionists identify as problematic. Yet the manufacture of disconnection can also be unappealing to technology platforms when the disconnection is economically disruptive. What ultimately determines whether the technology-based culture of disconnection is threatening to the attention economy is where the disconnective practice, or mode of disengagement, takes place, and who the affected parties are. In the following section, I focus on where the disconnection takes place in order to summarise what the manufacture of disconnection actually resists and how the manufacture of disconnection has transformed the attention economy and 
culture of connectivity. I close the thesis by suggesting a future direction for disconnection studies.

\subsection{Disconnecting by inhabiting digital spaces and subject positions}

This thesis has investigated how disconnection is produced by Silicon Valley technologists, or what I have referred to in this thesis as Silicon Valley disconnectionists. I have departed from earlier studies of disconnection that normatively define disconnection or suggest avoiding the technological side of disconnection, and instead sought to understand how disconnection can occur with technology or technologically driven practices. Specifically, I have identified and analysed new types of digital spaces and subject positions that enable smartphone and internet users to undertake disconnective practice. These spaces and subject positions are digital architectures designed by Silicon Valley disconnectionists for users to disconnect from aspects of the internet or user behaviours that are associated with the attention economy or culture of connectivity. I came to these conclusions by answering my first research question: What types of relations do technologies that manufacture disconnection produce? Drawing upon a late Foucauldian theoretical framework on power, technology and resistance, and the walkthrough method (Light, Burgess, and Duguay 2016), I analysed five technologies of disconnection as producing new relations of space and self. The spatial relations included a rehabilitative space that provides a convenient escape from smartphone connectivity, and a sanctuary that provides temporary immunity from dataveillance. In Part II I discussed how these disconnected digital spaces provide a contrast to networked or connected spaces and protect inhabitants from commercial surveillance practices. Distinct from disconnected spaces are subject positions for the purposes of disconnective practice. In Part III I analysed technologies of disconnection that enabled 
individual users to create a different relationship with their device, themselves, or specific apps (e.g., social media) that the user identifies as problematic. I identified three different subject positions that disconnected the user in some shape or form: the fixable self, the intentional self and the available self. The fixable self is a subject position that draws upon a mix of the behavioural sciences and software development, viewing the brain and user behaviour as simultaneously irrational and fixable. Following this, I outlined the intentional self as a subject position that derives from Silicon Valley counterculture that favours goalorientated living over spontaneity. Lastly, I argued in chapter seven that the available self is produced by hegemonic discourses of femininity in response to the perception that certain users, such as women, cannot disconnect as easily as others.

My second research question sought to more deeply explore the tensions between the manufacture of disconnection and the attention economy, asking: How do these relations implicate resistance to the attention economy and culture of connectivity? In general, I argued throughout the thesis that these relations of space and self render disconnection into a mechanism for platforms and users to reorganise the surrounding digital environment. By doing so, I have tried to unveil the politics of Silicon Valley resistance to the attention economy and highlight what is disconnected from and who can undertake such disconnection. I answer this question in greater detail below.

\section{1.1. Resisting communicative norms or behaviours}

The relations produced by the manufacture of disconnection orients resistance towards communicative behaviours that technology platforms and the attention economy have been accused of normalising (Lanier 2018). The burden of constant communicative availability and the obligation to socially reciprocate are communicative norms that have 
been linked to attention economy logics (Bosker 2016) and increases in stress and anxiety for smartphone users (Büchi, Festic, and Latzer 2019; Ling 2016; Reinecke et al. 2017). In chapter three, I argued that Ransomly contests these norms by creating a unique digital architecture that alters space and network protocols to enable users to disconnect while remaining in environments that have access to the internet. Inside the disconnected space produced by Ransomly, users can become unavailable to their networks and call to question communicative norms that are associated with ubiquitous internet access. Similarly, in chapter six, I discussed how the smartphone application Siempo batches notifications which enables smartphone users to respond to text messages, emails and other communications on their own schedules. These features enable users to not always be available or obliged to respond and therefore resist the culture of constant connectivity.

The manufacture of disconnection also resists another communicative behaviour that technology platforms and the attention economy supposedly encourages: unconscious user scrolling. I analysed Space and Siempo, two technologies of disconnection that directly aim to prevent unconscious user scrolling by offering slightly different governance techniques. I argued that Space and the technique of stimulus devaluation produces a fixable subject position that transforms the individual user relationship to their habits. By inserting a delay between the user's unconscious habits and their behaviours, the fixable self is trained by Space to become 'indistractable' (Eyal 2019) and ready to resist the lures of the attention economy. Similarly, I observed how Siempo draws upon the Silicon Valley counterculture of dropping out to reorganise the smartphone home screen and encourage intentional smartphone use. 


\section{1.2. The burden of change is on the individual}

Resistance to the attention economy is also positioned as an issue for individuals to undertake. Within my Foucauldian theoretical framework, I argued that the manufacture of disconnection enables users to have a sense of control in the process of being turned into a manageable subject in the attention economy. The fixable self and intentional self subject positions offer potentially empowering experiences for users with an opportunity to transform their relationship with their device, and their technology-based habits. Yet in both instances, the impetus is firmly on the individual user to resist the attention economy. The fixable self locates the issue of smartphone addiction as within the user (as opposed to produced by persuasive technology design) and positions the user as a subject to be disciplined (Guyard and Kaun 2018). Similarly, Siempo situates the issue of resistance at the level of digital lifestyle or consumption: what apps users should open, spend time on, and so on. Unconscious digital usage is framed as inauthentic behaviour and intentional behaviour as an aspirational mode of digital behaviour. In this sense, the manufacture of disconnection reinforces the political ideology of neoliberalism, turning the social problem of widespread persuasive design into a personal issue with market solutions for individuals to download. The Lilspace app presents disconnection as inextricably tied up with consumerist choices, where users are encouraged to disconnect from their phones to receive discounts on consumer items or experiences, further extending neoliberal logic and market principles into the realm of disconnective practice.

\subsubsection{Disconnection as governance}

I observed that the manufacture of disconnection often offers resistance to the attention economy via enhanced means to self-govern smartphone use. While the fixable and 
intentional subject positions disconnect the user from the habit of unconscious scrolling, both subjugate the user to additional governance methods that subtly discipline the user. The intentional self produced by Siempo requires the user to act intentionally all the time while on their device, disconnecting them from spontaneity or serendipitous smartphone play. I suggested Siempo is a productivity app that is dressed in wellness packaging and similarly positions the app as a protector of attention as opposed to an appropriator of attention. I also deconstructed how Space redeploys persuasive design in the form of a governance technique called stimulus devaluation to assist the user to 'fix' or break the habit of unconsciously opening flagged third party apps. Both Space and Siempo suggest that the problem of unconscious scrolling is determined by the type of interaction a user can have with technology, where the user subjugates themselves to further governance techniques instead of resisting technologised governance more broadly. It is this extension of the smartphone user-app relationship that provides a reason why Apple and Google might have adopted similar functionalities for their users to manage their technological habits in Screen Timeand Digital Wellbeing. Users are encouraged to increasingly offload responsibility onto technology and trust it to make decisions on their behalf. To this end, the manufacture of disconnection has transformed the attention economy by modelling how technologies can act as protectors of attention and how users can have an enhanced governance-type relationship with their device. By locating the disconnect as between the user and their technology habits as opposed to, say, the technology platform itself, the manufacture of disconnection creates a new category of governance functionalities, products and services for the technology industry that could provide a 'socio-economic lubricant' (Light and Cassidy 2014) to ensure continued user participation with the platform. 


\subsubsection{Disconnection from governance}

Sometimes, the manufacture of disconnection resists governance. A dominant governance method that the manufacture of disconnection can resist is dataveillance, or the collection and analysis of data about user activities to shape the users' online experience. In chapter four, I argued that The Disconnect shifts its readers off the network, providing them temporary immunity from commercial surveillance practices. The Disconnect demonstrates that disconnecting from the attention economy is not always about resisting the technological, but rather about the dominant logics that undergird the technological, such as surveillance or governing the user. By forcing its reader to disable their internet connection, The Disconnect provides a type of intellectual privacy where readers can engage with content on their own terms and not be distracted by advertisements or be prompted to consider datadriven functionalities, such as the top highlights functionality that has become common to ereading. Disconnecting from the profit motive underlying the digital economy that demands our attention is less appealing to the attention economy, where using data-driven analytics that provide detailed information about readership has become central to content creation and expected by commercial clients such as advertisers (Tandoc 2014). The Disconnect presents an alternative model of distributing and presenting content to online readers. While The Disconnect garnered mainstream attention, there is no evidence that other content creators have adopted its methods. For now, The Disconnect remains an outsider to dominant models of content distribution and presentation in the attention economy, and the totalising logic of dataveillance. 


\section{1.5. Moralising resistance}

The manufacture of disconnection can offer resistance to the attention economy in a way that inscribes new 'truths' onto users. Some of the technologies of disconnection presented resistance as a moral act, shifting disconnection into the realm of normalisation. I argued that Ransom/ymoralises the differences of a disconnected space to convince smartphone users that a disconnected space is worth their time. The alternative ordering of a disconnected space stabilises the social order of digital space and subjects users to new moral regimes of power. Siempo also moralises disconnection by claiming that downloading an intentional interface will improve the wellbeing of its users. Ransomly's claim that occupying a disconnected space is best for a user's 'humanity' or Siempo's suggestion that their intentional interface will improve the wellbeing for their users are not grounded in empirical evidence. More than that, a number of studies dispute any causality between screens, social media and mental ill health (Orben and Przybylski 2019; Reer, Tang, and Quandt 2019). In some instances, the manufacture of disconnection builds upon societal moral panic about appropriate smartphone usage (Furedi 2016; Jurgenson 2013, 2016) and seeks to impose normative, but perhaps arbitrary, limits to determine 'healthy use'.

The normalisation of disconnection is another reason the manufacture of disconnection has been embraced by technology platforms. As I have argued with others elsewhere, associating digital architectures and subject positions with notions of wellbeing generates new exclusionary norms in relation to 'healthy' or 'humane' technology practices (Lacey, Caudwell, and Beattie 2019) and provides an opportunity for Silicon Valley technology companies to position themselves as providing a new type of social good and forestall independent regulation of their platforms (Beattie and Daubs 2019). The enrolment of technologies of the self into regimes of normalisation and control has been raised as a point 
of concern (Bergen and Verbeek 2020) but largely neglected as a scholarly enquiry. The manufacture of disconnection generates new norms in relation to technology use and has undergirded the ethical and humanist turn in the Silicon Valley industry, a movement which has been criticised as insular and apolitical (Tarnoff and Weigel 2018).

\section{1.6. Extends the power of technologists in the attention economy}

The manufacture of disconnection also extends and obfuscates the power of technologists in the attention economy. In chapter five, I argued that persuasive technology, a discipline that has become integral to the attention economy, is positioned as an individual self-care issue, and neglects to attend to the additional power that is granted to persuasive technologists who can use their expertise to make technology-based connecting and disconnecting more persuasive. Technologies of disconnection also provide data on practices that were previously analogue (e.g., habits that users wish to break or apps they want to use less of) and could be profitable to a range of actors including employers or insurers. The shift of technology platforms into manufacturing disconnection has been detrimental to some smaller Silicon Valley disconnectionists. Apple has squeezed out several disconnection or wellbeing entrepreneurs who depend on access to Apple's iOS app store to distribute their product or service. As Apple governs the app store, Apple alone decides which apps meet their criteria, and the company has shut out some independent technologies of disconnection like Siempo and Space (Nicas 2019). Instead of analysing the possible limits of harms of attention economy logics in certain contexts, the manufacture of disconnection has offered individual solutions and new user/technology relations for the likes of Apple and Google to replicate. 


\section{1.7. Reinforces inequalities of disconnection}

Finally, the manufacture of disconnection reinforces gender inequities with regards to who can disconnect and on what terms. In chapter seven, I argued that there is a dominant model of disconnecting embodied in the archetype of the Man Alone-an unencumbered subject position free to cut their ties at whim. In response to the Man Alone, I identified a type of disconnecting subject position that I called the available self. The available self only gets to undertake the weakest type of disconnection; the Lilspace user is not socially disconnected from other users but encouraged to undertake a disconnection with other users and/or for a cause or event. In addition, the available self is brought closer to traditional ideas about gender roles. Rather than getting to disconnect on the terms that the Man Alone is allowed and cut social ties at whim, the available self is encouraged to remain tethered to its social obligations, expectations and role. The available self suggests that only certain people get to enjoy the transformative benefits of a fuller disconnection. Full participation in the technology-driven culture of disconnection remains only available to a select few people.

These features of the manufacture of disconnection contribute towards a technologybased culture of disconnection. In this Silicon Valley culture of disconnection, users are encouraged to access rehabilitative and sanctuary type disconnected spaces or inhabit fixable, intentional, and available subject positions to resist the attention economy. I have observed how these digital architectures disconnect users from communicative norms such as constant connectivity or undesirable digital consumerist habits like mindless scrolling. Resistance to the attention economy is shaped around the perceived lifestyle needs of individual users such as reducing distractions or excessive screen time that harm productivity or the development of the self, rather than about user data collection. In addition, I have highlighted how some technologies of disconnection offer enhanced self-governance 
techniques and utilise moralistic ideals that provide a therapeutic vocation for persuasive app designers and create a new category of products and services for technology platforms like Apple and Google to shift into. The manufacture of disconnection even keeps intact preexisting power structures, such as who gets to critique the attention economy, or disconnect from other people and on what terms. I therefore conclude that the manufacture of disconnection largely challenges routine living in the attention economy without dismantling key structures of the attention economy, such as the collection of user data and unregulated persuasive design. As a result, a key argument of this thesis is that the manufacture of disconnection is largely a false or ostensible challenge that works to support, rather than destabilise the attention economy.

I do, however, believe that the manufacture of disconnection can threaten the structures of the attention economy when the user is disconnected from the logics that undergird the attention economy. The Disconnect, is an example where the user is disconnected from dataveillance logics that are central to the attention economy. However, as accessing and contributing to The Disconnect currently requires a significant amount of privilege, disconnecting users from the logics of the attention economy is the exception that proves the rule. It remains to be seen how disconnecting a user from the logics of the attention economy can be translated to broader, or more general contexts. For now, I believe that the viability of the manufacture of disconnection as a mode of resistance to fundamentally change the attention economy largely depends on where the disconnect takes place and what is being resisted. Disconnecting a user from their technology-based habits has the effect of extending self-surveillance or governance, while disconnecting a user from the logics of the attention economy resists surveillance. While the manufacture of 
disconnection appears to be ambivalent with regards to governance, we can now distinguish relations that are desirable to technology platforms from architectures that are not.

\subsection{The way forward and future research possibilities}

This thesis has analysed the manufacture of disconnection and the advancement of a technology-driven culture of disconnection. By uncovering digital architectures that are produced by the manufacture of disconnection, I have shown that disconnection scholarship can explore and critically assess the reconstruction of our digital environment and selves. Since beginning this PhD in 2017, the number of technologies of disconnection has grown exponentially and disconnecting from the internet has grown more popular. Individual technologies of disconnection will come and go, but the new relations generated by the manufacture of disconnection will linger for much longer. Technologies like Ransomlymay eventually disappear from the Google Play store but the idea of a rehabilitative disconnected space continues to build momentum in the form of Wi-Fi free cafés and smartphone-free classrooms or entertainment venues (Hutchins 2016; Merlino 2019; Smith and Keane 2019). While there are a growing number of tourism studies that claim disconnected spaces attract certain holiday-goers like backpackers or business executives (Dickinson, Hibbert, and Filimonau 2016; Rosenberg 2019), disconnection scholars could assess the appeal and implications of a disconnected space in the context of education or live entertainment.

There is also the task of further denaturalising the claims that Ransomlyand Siempo make. In this thesis, I have argued that the moralisation of disconnection is not based on empirical evidence but subjective concerns about the harms of connectivity on selfrepresentation and development. An alternative approach to further question the notion that disconnection is a healthy practice could be to adopt a Foucauldian genealogical method and 
trace the history of the distracted or digitally ill subject. Influential psychologists and social scientists such as William James ([1893] 1950) and, more recently, Daniel Kahneman (2011) have offered theories around the distracted brain and cognition that have recently influenced the design of some technologies of disconnection (Lyngs et al. 2019; Lyngs et al. 2020). There are also historical technologies such as the swear jar (Plaut 2015) that have been incentivising the behaviour of the people long before digital media technologies and the internet. A genealogical approach could further explore the social construction of the distracted or impulsive subject which will lead to further discussions over how 'digital wellbeing' or 'digital health' could be conceived otherwise.

Some Silicon Valley disconnectionists that I researched have been given a bigger platform because of their inventions. In chapter five I noted that Space has been purchased by Thrive Global, with the technologists behind Space appointed as 'behavioural executives' (Huffington 2019). One way to critically interrogate a profession like a behavioural executive could be to ground a study in Foucault's ([1976] 1990, 138) concept of biopolitics, or the political logic that administers life and populations as its subject: "to ensure, sustain, and multiply life, to put this life in order." With flexible working on the rise following the COVID-19 pandemic and work/leisure boundaries at risk of breaking down further, the demand to put one's digital life in order could increase. There are a growing number of sectors interested in the biopolitics of disconnection: social media platforms that view user disconnection as a threat to their business (Karppi 2018), employers who view disconnecting from the internet as an opportunity for their employees to self-discipline and enhance productivity (Gregg 2018; Guyard and Kaun 2018), and insurance and health and wellbeing organisations that instrumentalise data about disconnective practices in their products and services (Zuboff 2019). Scholarship in this vein could analyse the benefits and risks of offering enhanced 
governance methods to these sectors. A larger question that could be asked is whether a biopolitical project of disconnection could be democratic and serve the interests of people, rather than behavioural executives and the operators of the technologies of disconnection.

The legacy of the intentional self and Siempo, with its idea to batch notifications and deploy persuasive design to nudge users off their devices remains in Apple's Screen Time. Affect is a concept that I have briefly discussed throughout this thesis, and could be explored more substantially to understand the affective dimensions of disconnecting with technology. If platforms and companies instrumentalise affect for commercial purposes (Munn 2020; Stark 2018), then an emphasis on the affective pull of disconnective practice in technologies such as Screen Time could reveal whether technology-enabled disconnection extends such logic and revitalise media effects debates about the agency of users. I explored the gender inequities of disconnection in Lilspace, but there are likely other socioeconomic, ethnic or cultural factors that preclude people from being able to disconnect and would further develop the argument that disconnecting from the internet is a privilege that not many users of the internet can undertake (Beattie 2018a).

While a major finding of this thesis was how individualistic the manufacture of disconnection is, disconnection scholars should not assume that neoliberalism is a totalising reality from which disconnective practices cannot escape. One way to spotlight collective attempts to disconnect users from the attention economy could be to analyse calls to regulate the architecture of online spaces and persuasive technology (see ICO 2019) or design codes of conduct or standards (see Shamonsky 2018). In a similar manner to how Nieborg and Poell (2018) examined the 'platformisation' of cultural industries such as journalism and video games, a political economy approach could also take up the question of the platformisation of disconnection in more detail. Future research could examine the 
potential implications of the fact that the gatekeepers for mobile applications, Google and Apple, can largely control the manufacture of disconnection by determining what technologies of disconnection make it to the app store for people to use.

This thesis opened with a quote from Silicon Valley technologist Loren Brichter about his pull-to-refresh functionality, concerns about contributing to an 'addictive' social media and a perceived growth in maturity as a technology developer. Through this work I have revealed how Silicon Valley disconnectionists have responded by creating a technology-based culture of disconnection. I studied Silicon Valley disconnectionists and their technologies of disconnection as attempts to resist the attention economy. I found that these technologies largely disconnect a user from their undesirable smartphone behaviours or communicative norms that are believed to be problematic. Aside from when a user is actually disconnected from the logics of the attention economy, I concluded that the manufacture of disconnection extends, rather than fundamentally challenges, the attention economy. Nonetheless, the manufacture of disconnection signals a critical period in the history of Silicon Valley. By pivoting away from constant connectivity, Silicon Valley disconnectionists have pioneered new disconnective relationships users can have with technology. In doing so, they have transformed our digital environments and selves. Brichter may claim he has matured as a technologist, but our understanding of how disconnection is produced by technologists is only beginning. 


\section{Reference list}

6 P, and Ben Jupp. 2001. Divided by information? The "digital divide" and the implications of the new meritocracy. London: Demos.

Addis, Michael E., and James R. Mahalik. 2003. "Men, Masculinity, and the Contexts of Help Seeking." American Psychologist 58 (1): 5-14. https://doi.org/10.1037/0003066X.58.1.5.

Aiken, Mary. 2016. The Cyber Effect: A Pioneering Cyberpsychologist Explains How Human Behavior Changes Online. London: Hachette UK.

Allen, Brenda. 2011. "The Man Alone, the Black Sheep and the Bad Apple : Squeaky Wheels of New Zealand Cinema." MediaNZ12 (2): 87-109. https://doi.org/10.11157/medianzvol12iss2id41.

Alter, Adam. 2017. Irresistible: Why We Can't Stop Checking, Scrolling, Clicking and Watching. London: The Bodley Head.

Amazeen, Michelle A. 2019. "News in an Era of Content Confusion: Effects of News Use Motivations and Context on Native Advertising and Digital News Perceptions." Journalism \& Mass Communication Quarterly 97 (1): 161-187. https://doi.org/10.1177/1077699019886589.

Ames, Morgan G. 2013. "Managing mobile multitasking: the culture of iPhones on stanford campus." In Proceedings of the 2013 Conference on Computer Supported Cooperative Work, 1487-1498. San Antonio: ACM. https://doi.org/10.1145/2441776.2441945.

Andrejevic, Mark. 2007. "Surveillance in the Digital Enclosure." The Communication Review 10 (4): 295-317. https://doi.org/10.1080/10714420701715365.

Apple. 2018. "iOS 12 introduces new features to reduce interruptions and manage Screen Time ". Apple. June 5 2018. https://www.apple.com/nz/newsroom/2018/06/ios-12introduces-new-features-to-reduce-interruptions-and-manage-screen-time/.

Ball, James. 2017. Post-Truth: How Bullshit Conquered the World. London: Biteback Publishing.

Bandura, Albert. 1977. Social learning theory. Englewood Cliffs: Prentice Hall.

Banet-Weiser, Sarah. 2007. "What's Your Flava? Race and Postfeminism in Media Culture." In Interrogating Postfeminism: Gender and the Politics of Popular Culture, edited by Diane Negra and Yvonne Tasker, 201-226. Durham: Duke University Press.

Banks, David. 2015. "Lines of power: Availability to networks as a social phenomenon." First Monday 20 (11). https://doi.org/10.5210/fm.v20i11.6283.

Barbedette, Gilles. 1982. "The Social Triumph of the Sexual Will: A Conversation with Michel Foucault." Translated by Brendan Lemon, Christopher Street, 6 (4): 36-41. 
Barbrook, Richard, and Andy Cameron. 1996. "The Californian ideology." Science as Culture 6 (1): 44-72. https://doi.org/10.1080/09505439609526455.

Barker, Gary. 2005. Dying to be Men: Youth, Masculinity and Social Exclusion (Sexuality, Culture and Health Book). London: Routledge.

Baron, Naomi S. 2011. "Concerns about mobile phones: A cross-national study." First Monday 16 (8). https://firstmonday.org/ojs/index.php/fm/article/view/3335/3032.

Bartholomew, Mark. 2017. Adcreep: The Case Against Modern Marketing. Stanford: Stanford Law Books.

Bauer, Martin. W. 1995. "'Technophobia': a misleading conception of resistance to new technology." In Resistance to New Technology: Nuclear Power, Information Technology and Biotechnology, edited by Martin. W. Bauer, 97-124. Cambridge, UK: Cambridge University.

Baym, Nancy K., Kelly B. Wagman, and Christopher J. Persaud. 2020. "Mindfully Scrolling: Rethinking Facebook After Time Deactivated." Social Media + Society 6 (2). https://doi.org/10.1177/2056305120919105.

Beal, Vangie. n.d. "Android Launcher." Webopedia. Accessed July 21, 2020. https://www.webopedia.com/TERM/A/android_launcher.html.

Beattie, Alex, and Michael Daubs. "Framing Digital Wellbeing as a Social Good." Paper presented at the Australasian Association for the History, Philosophy, and Social Studies of Science Conference, Wellington, New Zealand, 12-13 November 2019.

Beattie, Alex. 2018a. "Escape: the next digital divide." The Disconnect 1. February 2018. https://thedisconnect.co/one/.

Beattie, Alex. 2018b. "Out of Network." Real Life, 29 May, 2018. https://reallifemag.com/outof-network/.

Beckett, Angharad E., Paul Bagguley, and Tom Campbell. 2017. "Foucault, social movements and heterotopic horizons: rupturing the order of things." Social Movement Studies 16 (2): 169-181. https://doi.org/10.1080/14742837.2016.1252666.

Behrent, Michael C. 2013. "Foucault and Technology." History and Technology 29 (1): 54-104. https://doi.org/10.1080/07341512.2013.780351.

Bennett, Catherine. 2019. "Wife-tracking apps are one sign of Saudi Arabia's vile regime. Others include crucifixion...." The Guardian, June 16, 2019. https://www.theguardian.com/commentisfree/2019/apr/28/wife-tracking-appssaudi-arabias-vile-regime-crucifixion.

Bennett, Tony, Francis Dodsworth, Greg Noble, Mary Poovey, and Megan Watkins. 2013. "Habit and Habituation: Governance and the Social." Body \& Society 19 (2-3): 3-29. https://doi.org/10.1177/17357034X13485881. 
Benson, Dale. 1999. "New Zealand's Existentialist Men Alone: A Survey of Their Development from the 1890s until the 1970s ". Deepsouth 4 (2). https://www.otago.ac.nz/deepsouth/1198/MenAlone.html.

Berdichevsky, Daniel, and Erik Neuenschwander. 1999. "Toward an Ethics of Persuasive Technology." Communications of the ACM 42 (5): 51-58.

Bergen, Jan Peter, and Peter-Paul Verbeek. 2020. "To-Do Is to Be: Foucault, Levinas, and Technologically Mediated Subjectivation." Philosophy \& Technology. https://doi.org/10.1007/s13347-019-00390-7.

Berry, David M. 2011. "The Computational Turn: Thinking About the Digital Humanities." Culture Machine 12: 1-22. http://svr91.edns1.com/ culturem/index.php/cm/issue/view/23.

Berry, Marsha. 2008. "Locative media: geoplaced tactics of resistance." International Journal of Performance Arts and Digital Media 4 (2-3): 101-116.

Binkley, Sam. 2014. Happiness as Enterprise: An Essay on Neoliberal Life. Albany: SUNY Press.

Black, K. 1986. "The information poor." RQ25 (4): 517-518.

Bolin, Chris, and Clayton d'Arnault. 2018. "Rethinking Digitality with Nathan Jurgenson." The Disconnect 2. September 2018. https://thedisconnect.co/two/rethinking-digitality/.

Bolin, Chris. 2018. "Offline Only." 29 October, 2018. https://chris.bolin.co/offline/.

Bollmer, Grant. 2016. Inhuman Networks: Social Media and the Archaeology of Connection. New York: Bloomsbury.

Bolt, David, and Ray Crawford. 2000. Digital Divide: Computers and Our Children's Future. New York: TV Books.

Borgmann, Albert. 1984. Technology and the Character of Contemporary Life: A Philosophical Inquiry. Chicago: University of Chicago Press.

Bosker, Bianca. 2016. "The Binge Breaker: Tristan Harris believes Silicon Valley is addicting us to our phones. He's determined to make it stop." The Atlantic. November 2016. https://www.theatlantic.com/magazine/archive/2016/11/the-binge-breaker/501122/.

Boundless Mind. n.d. "Boundless Mind." Accessed 10 January, 2019. https://www.boundless.ai/.

Boyer, M. Christine. 2008. "The many mirrors of Foucault and their architectural reflections." In Heterotopia and the City: Public Space in a Postcivil Society, edited by Michiel Dehaene and Lieven De Cauter, 53-74. Abingdon: Routledge.

Bratton, Benjamin H. 2015. The Stack: On Software and Sovereignty. Cambridge, MA: MIT Press. 
Braun, Virginia, Victoria Clarke, and Debra Gray. 2017. Collecting Qualitative Data: A Practical Guide to Textual, Media and Virtual Techniques. Cambridge, UK: Cambridge University Press.

Braun, Virginia, Victoria Clarke, Nikki Hayfield, and Gareth Terry. 2019. "Thematic Analysis." In Handbook of Research Methods in Health Social Sciences, edited by Pranee Liamputtong, 843-860. Singapore: Springer.

Brignall, Harry. n.d. "Dark Patterns." Dark Patterns. Accessed 21 July, 2017. https://darkpatterns.org/.

Brinkmann, Svend, and Steinar Kvale. 2014. InterViews: Learning the Craft of Qualitative Research Interviewing. 2nd ed. New York: Sage.

Brown, Allison J. 2020. "'Should I Stay or Should I Leave?": Exploring (Dis)continued Facebook Use After the Cambridge Analytica Scandal." Social Media + Society 6 (1): https://doi.org/10.1177/2056305120913884.

Bruns, Axel. 2008. Blogs, Wikipedia, Second Life, and Beyond: From Production to Produsage. New York: Peter Lang.

Brunton, Finn, and Helen Nissenbaum. 2011. "Vernacular resistance to data collection and analysis: A political theory of obfuscation." First Monday 16 (5) https://firstmonday.org/article/view/3493/2955.

Bucher, Taina. 2012. "Want to be on the top? Algorithmic power and the threat of invisibility on Facebook." New Media \& Society 14 (7): 1164-1180.

https://doi.org/10.1177/1461444812440159.

Bucher, Taina. 2020. "Nothing to disconnect from? Being singular plural in an age of machine learning." Media, Culture \& Society 42 (4): 610-617 https://doi.org/10.1177/0163443720914028.

Büchi, Moritz, Noemi Festic, and Michael Latzer. 2019. "Digital Overuse and Subjective WellBeing in a Digitized Society." Social Media + Society 5 (4): https://doi.org/10.1177/2056305119886031.

Burelle, Ainsleigh. 2017. "What's in a Community Builder? Going Beyond the Buzzwords." Medium. 14 February, 2017. https://blog.communitybuilders.io/whats-in-acommunity-builder-going-beyond-the-buzzwords-fa7e3c36e778.

Butler, Judith. 1997. The Psychic Life of Power: Theories in Subjection. Stanford: Stanford University Press.

Butler, Judith. 1999. Gender Trouble: Feminism and the Subversion of Identity. New York: Routledge.

Calvo, Rafael A, and Dorian Peters. 2014. Positive Computing: Technology for Wellbeing and Human Potential. Cambridge, MA: MIT Press. 
Capsana. 2019. "Pause." Capsana. Accessed 10 May, 2019.

https://pausetonecran.com/en/manifest-home/.

Cardullo, Paolo, Cesare Di Feliciantonio, and Rob Kitchin, eds. 2019. The Right to the Smart City. Bingley: Emerald Publishing Limited.

Carlquist, Erik, Lin Prøitz, and Katrina Roen. 2019. "Streams of fun and cringe: talking about Snapchat as mediated affective practice." Subjectivity 12: 228-246. https://doi.org/10.1057/s41286-019-00074-9.

Carr, Nicholas. 2011. The Shallows: What the Internet Is Doing to Our Brains. New York: W. W. Norton \& Company.

Carter, Eric D. 2015. "Making the Blue Zones: Neoliberalism and nudges in public health promotion." Social Science \& Medicine 133: 374-382. https://doi.org/10.1016/j.socscimed.2015.01.019.

Cartwright, Darren. 2018. "'Digital detox' holidays are on the rise." Stuff. July 11, 2018. https://www.stuff.co.nz/travel/news/105391838/digital-detox-holidays-are-on-therise.

Case, Amber. 2015. Calm Technology: Principles and Patterns for Non-Intrusive Design. Sebastopol: O'Reilly Media.

Cash, Ivan. 2015. "No Tech-Zone" Ivan Cash http://www.ivan.cash/no-tech-zone.

Chade-Meng, Tan. 2012. Search Inside Yourself: The Unexpected Path to Achieving Success, Happiness (and World Peace). New York: HarperCollins.

Chandler, David, and Julian Reid. 2016. The Neoliberal Subject: Resilience, Adaption and Vulnerability. Lanham: Rowman \& Littlefield.

Chang, Emily. 2018. Brotopia: Breaking Up the Boys' Club of Silicon Valley. New York: Portfolio.

Cheney-Lippold, John. 2011. "A New Algorithmic Identity: Soft Biopolitics and the Modulation of Control." Theory, Culture \& Society 28 (6): 164-181. https://doi.org/10.1177/0263276411424420.

Cheney-Lippold, John. 2017. We Are Data: Algorithms and the Making of our Digital Selves. New York: New York University Press.

Chun, Wendy Hui Kyong. 2006. Control and Freedom: Power and Paranoia in the Age of Fiber Optics. Cambridge, MA: MIT Press.

Chun, Wendy Hui Kyong. 2016. Updating to Remain the Same: Habitual New Media. Cambridge, MA: MIT Press. 
Churchill, Sue, Donna Jessop, and Paul Sparks. 2008. "Impulsive and/or planned behaviour: Can impulsivity contribute to the predictive utility of the theory of planned behaviour?" Social Psychology 47 (4): 631-646.

Citton, Yves. 2017. The Ecology of Attention. Hoboken: Wiley.

Cohen, Peter, Robert Hahn, Jonathan Hall, Steven Levitt, and Robert Metcalfe. 2016. "Using Big Data to Estimate Consumer Surplus: The Case of Uber." National Bureau of Economic Research. http://www.nber.org/papers/w22627.

Combs, T Dalton, and Ramsay A Brown. 2018. Digital Behavioral Design. Venice Beach: Boundless Mind.

Connell, Raewyn W. 1987. Gender and Power: Society, the Person and Sexual Politics. Sydney: Allen \& Unwin.

Connell, Raewyn W. 1995. Masculinities. Sydney: Allen \& Unwin.

Connell, Raewyn W., and James W. Messerschmidt. 2005. "Hegemonic Masculinity: Rethinking the Concept." Gender \& Society 19 (6): 829-859.

https://doi.org/10.1177/0891243205278639.

Couldry, Nick, and Andreas Hepp. 2017. The Mediated Construction of Reality. Cambridge, UK: Polity Press.

Couldry, Nick, and Ulises Ali Mejias. 2019. The Costs of Connection: How Data Is Colonizing Human Life and Appropriating It For Capitalism. Stanford: Stanford University Press.

Courtwright, David T. 2019. The Age of Addiction: How Bad Habits Became Big Business. Cambridge, MA: Harvard University Press.

Crogan, Patrick, and Samuel Kinsley. 2012. "Paying Attention: Towards a Critique of the Attention Economy." Culture Machine 13: 1-29. https://culturemachine.net/wpcontent/uploads/2019/01/463-1025-1-PB.pdf.

Crunchbase. n.d. "Thrive Global." Accessed 21 July, 2020.

https://www.crunchbase.com/organization/thrive-global.

Cushman, Fiery. 2015. "Deconstructing intent to reconstruct morality." Current Opinion in Psychology6: 97-103. https://doi.org/10.1016/j.copsyc.2015.06.003.

Das, Salil K. 2003. "Harmful health effects of cigarette smoking." Molecular and Cellular Biochemistry 253 (1): 159-165. https://doi.org/10.1023/a:1026024829294.

Dean, Jodi. 2005. "Communicative Capitalism: Circulation and the Foreclosure of Politics." Cultural Politics 1 (1): 51-74. https://doi.org/10.2752/174321905778054845.

deBruler, Cyndy Hess. 2017. Intentional Eating: An Easy, Mindful Approach to Dietary Wellness for Increased Vitality, Weight Control, Chronic Disease Management and Stress Reduction. Bloomington: Balboa Press. 
Deleuze, Gilles, and Félix Guattari. 1990. "Gilles Deleuze and Felix Guattari on Anti-Oedipus." In Negotiations, 1972-1990, edited by Gilles Deleuze, 13-25. New York: Columbia University Press.

Deleuze, Gilles. 1992. "Postscript on the Societies of Control." October59: 3-7. www.jstor.org/stable/778828.

Deseriis, Marco. 2012. "Improper names: Collective pseudonyms and multiple-use names as minor processes of subjectivation." Subjectivity 5 (2): 140-160.

https://doi.org/10.1057/sub.2012.3.

Deseriis, Marco. 2013. "Is Anonymous a New Form of Luddism? A Comparative Analysis of Industrial Machine Breaking, Computer Hacking, and Related Rhetorical Strategies." Radical History Review (117): 33-48. https://doi.org/10.1215/01636545-2210437.

Deuze, Mark. 2006. "Participation, Remediation, Bricolage: Considering Principal Components of a Digital Culture." The Information Society 22 (2): 63-75.

Dewe, Michaela, Jane Ogden, and Adrian Coyle. 2013. "The cigarette box as an advertising vehicle in the United Kingdom: A case for plain packaging." Journal of Health Psychology 20 (7): 954-962. https://doi.org/10.1177/1359105313504236.

Dickinson, Janet E., Julia F. Hibbert, and Viachaslau Filimonau. 2016. "Mobile technology and the tourist experience: (Dis)connection at the campsite." Tourism Management 57: 193-201. https://doi.org/10.1016/j.tourman.2016.06.005.

Dieter, Michael, Carolin Gerlitz, Anne Helmond, Nathaniel Tkacz, Fernando N. van der Vlist, and Esther Weltevrede. 2019. "Multi-Situated App Studies: Methods and Propositions." Social Media + Society 5 (2). https://doi.org/10.1177/2056305119846486.

Docherty, Niall. 2020. "Facebook's Ideal User: Healthy Habits, Social Capital, and the Politics of Well-Being Online." Social Media + Society 6 (2).

https://doi.org/10.1177/2056305120915606.

Doctor, Ronald D. 1991. "Information technologies and social equity: Confronting the revolution." Journal of the American Society for Information Science 42 (3): 216-228.

Dolan, Paul. 2014. Happiness By Design: Change What You Do, Not How You Think. New York: Avery Books.

Donne, John. 1988. No Man Is An Island. Edited by Helen Lush. London: Souvenir Press.

Dorrestijn, Steven, and Peter-Paul Verbeek. 2013. "Technology, wellbeing, and freedom: The legacy of utopian design." International Journal of Design 7 (3): 45-56.

Dougherty, Ian. 1996. Arawata Bill: the story of legendary gold prospector William James O'Leary. Auckland: Exisle Publishing. 
Driver, Susan. 2017. "An ethics of networked caring within young people's everyday lives."

Feminist Media Studies 17 (2): 297-301.

https://doi.org/10.1080/14680777.2017.1283745.

Duffy, Brooke Erin, and Emily Hund. 2015. "'Having it All" on Social Media: Entrepreneurial Femininity and Self-Branding Among Fashion Bloggers." Social Media + Society 1 (2): https://doi.org/10.1177/2056305115604337.

Duffy, Brooke Erin. 2015. (Not) Getting Paid to Do What You Love: Gender, Social Media, and Aspirational Work. New Haven: Yale University Press.

Duke, Éilish, and Christian. Montag. 2017. "Smartphone addiction, daily interruptions and selfreported productivity." Addict Behav Rep 6: 90-95.

https://doi.org/10.1016/j.abrep.2017.07.002.

Dunbar, R. I. M. 1992. "Neocortex size as a constraint on group size in primates." Journal of Human Evolution 22 (6): 469-493. https://doi.org/https://doi.org/10.1016/00472484(92)90081-J.

Dyer-Witherford, Nick. 1999. Cyber-Marx: Cycles and Circuits of Struggle in High Technology Capitalism. Chicago: University of Illnois Press.

Elden, Stuart. 2016. Foucault's Last Decade. Hoboken: Wiley.

Ellison, Nicole B., Charles Steinfield, and Cliff Lampe. 2007. "The Benefits of Facebook "Friends:" Social Capital and College Students' Use of Online Social Network Sites." Journal of Computer-Mediated Communication 12 (4): 1143-1168. https://doi.org/10.1111/j.1083-6101.2007.00367.x.

Ellul, Jacques. 1965. The Technological Society. London: Cape. First published in 1954.

English-Lueck, Jan. 2010. Being and Well-Being: Health and the Working Bodies of Silicon Valley. Stanford: Stanford University Press.

Ettlinger, Nancy. 2018. "Algorithmic affordances for productive resistance." Big Data \& Society 5 (1). https://doi.org/10.1177/2053951718771399.

Evans, Adrienne, Sarah Riley, and Avi Shankar. 2010. "Postfeminist Heterotopias: Negotiating 'Safe' and 'Seedy' in the British Sex Shop Space." European Journal of Women's Studies 17 (3): 211-229. https://doi.org/10.1177/1350506810368817.

Eyal, Nir. 2014. Hooked: How to Build Habit-Forming Products. New York: Portfolio.

Eyal, Nir. 2019. Indistractable: How to Control Your Attention and Choose Your Life. London: Bloomsbury.

Farman, Jason. 2011. Mobile Interface Theory: Embodied Space and Locative Media. New York: Routledge. 
Federici, Sylvia. 1995. "Wages Against Housework." In The Politics of Housework, edited by Ellen Malos, 187-194. New Clarion: Cheltenham.

Feenberg, Andrew. 1991. Critical Theory of Technology. New York: Oxford University Press.

Ferrer Conill, Raul. 2016. "Camouflaging Church as State." Journalism Studies 17 (7): 904-914. https://doi.org/10.1080/1461670X.2016.1165138.

Ferriss, Tim. 2011. The 4-Hour Work Week: Escape the 9-5, Live Anywhere and Join the New Rich. London: Ebury Press.

Fish, Adam. 2017. "Technology Retreats and the Politics of Social Media." triple C 15 (1): 355369. https://doi.org/10.31269/triplec.v15i1.807.

Fisher, Adam. 2018. Valley of Genius: The Uncensored History of Silicon Valley. New York: Twelve.

Flew, Terry. 2014. New Media. 4th ed. Oxford: Oxford University Press.

Floridi, Luciano. 2014. The Fourth Revolution: How the Infosphere is Reshaping Human Reality. Oxford: Oxford University Press.

Fogg, BJ. 2001. Persuasive Technology: Using Computers to Change What We Think and Do. Burlington: Morgan Kaufmann.

Fogg, BJ. 2019. Tiny Habits: The Small Changes That Change Everything. Boston: Houghton Mifflin Harcourt.

Foot, K. 2014. "The Online Emergence of Pushback on Social Media in the United States: A Historical Discourse Analysis." International Journal of Communication 8: 1313-1342.

Fotopoulou, Aristea. 2014. "Digital and networked by default? Women's organisations and the social imaginary of networked feminism." New Media \& Society 18 (6): 989-1005. https://doi.org/10.1177/1461444814552264.

Foucault, Michel. (1960) 1988. Madness and Civilization: A History of Insanity in the Age of Reason. London: Vintage.

Foucault, Michel. (1966) 1994. The Order of Things: An Archeology of the Human Sciences. London: Vintage.

Foucault, Michel. (1969) 1982. The Archaeology of Knowledge: And The Discourse on Language. London: Vintage.

Foucault, Michel. (1975) 1977. Discipline and Punish: The Birth of the Prison. London: Penguin Books.

Foucault, Michel. (1976) 1990. The History of Sexuality, Volume I: An Introduction. London: Vintage. 
Foucault, Michel. (1984) 1988. The History of Sexuality, Volume III: The Care of the Self. London: Vintage.

Foucault, Michel. (1984) 1990. The History of Sexuality, Volume II: The Use of Pleasure. London: Vintage.

Foucault, Michel. 1980. "Truth and Power." In Power/Knowledge: Selected Interviews and Other Writings 1927-1977, edited by Colin Gordon, 109-133. New York: Pantheon Books.

Foucault, Michel. 1982. "The Subject and Power." In Michel Foucault: Beyond Structuralism and Hermeneutics, edited by Hubert L Dreyfus and Paul Rabinow, 208-226. Brighton: The Harvester Press.

Foucault, Michel. 1986. "Of Other Spaces." Diacritics 16 (1): 22-27. Translated by Jay Miskowiec. https://doi.org/10.2307/464648.

Foucault, Michel. 1988. "Technologies of the Self." In Technologies of the Self: A Seminar with Michel Foucault, edited by Luther H Martin, Huck Gutman and Patrick H Hutton, 16-49. Amherst: The University of Massachusetts Press.

Foucault, Michel. 1993. "Space, power and knowledge." In The Cultural Studies Reader, edited by Simon During, 161-169. London: Routledge.

Foucault, Michel. 1997. "The ethics of the concern for self as a practice of freedom." In Ethics. Subjectivity and truth: Essential works of Foucault, 1954-1984, edited by Paul Rabinow, 281-302. New York: The New Press.

Foucault, Michel. 2005. The Hermeneutics of the Subject: Lectures at the Collège De France, 1981-1982. New York: Palgrave-Macmillan.

Foucault, Michel. 2008. The Birth of Biopolitics: Lectures at the Collège de France, 1978-1979. Translated and edited by Graham Burchell and Arnold Davidson. London: Palgrave Macmillan.

Foucault, Michel. 2009. Le Corps Utopique - Les Hétérotopies. Clamecy: Éditions Lignes.

Foucault, Michel. 2018. The History of Sexuality, Volume IV: Confessions of the Flesh. Edited by Frédéric Gros. Paris: Gallimard.

Frischmann, Brett and Evan Selinger. 2018. Re-Engineering Humanity. Cambridge, UK: Cambridge University Press.

Frith, Jordan. 2015. Smartphones as Locative Media. New York: Wiley.

Fuchs, Christian. 2012. "Political Economy and Surveillance Theory." Critical Sociology 39 (5): 671-687. https://doi.org/10.1177/0896920511435710.

Furedi, Frank. 2016. "Moral Panic and Reading: Early Elite Anxieties About the Media Effect." Cultural Sociology 10 (4): 523-537. 
Galloway, Alexander, and Eugene Thacker. 2007. The Exploit: A Theory of Networks. Edited by Eugene Thacker. Minneapolis: University of Minnesota Press.

Galloway, Alexander. 2012. The Interface Effect. New York: Wiley.

Gauthier, François 2013. "The Enchantments of Consumer Capitalism: Beyond Belief at the Burning Man Festival." In Religion in Consumer Society: Brands, Consumers and Markets, edited by François Martikainen Gauthier, Tuomas 143-158. Farnham: Ashgate.

Gehl, Robert William. 2013. "What's on your mind? Social media monopolies and noopower." First Monday 18 (3). https://firstmonday.org/ojs/index.php/fm/article/view/4618.

Gerbaudo, Paolo. 2013. "The 'Kill Switch' as 'Suicide Switch': Mobilizing Side Effects of Mubarak's Communication Blackout." Westminster Papers in Communication and Culture 9 (2): 25-46. http://doi.org/10.16997/wpcc.165.

Gergen, Kenneth J, and Mary M. Gergen. 2008. "Social Constructionism." In The SAGE Encyclopedia of Qualitative Research Methods, edited by Lisa M. Given. https://doi.org/10.4135/9781412963909.

Gergen, Kenneth J. 2002. "The Challenge of Absent Presence." In Perpetual Contact: Mobile Communication, Private Talk, Public Performance, edited by James E. Katz and Mark A. Aakhus, 227-241. New York: Cambridge University Press.

Giaxoglou, Korina, and Katrin Döveling. 2018. "Mediatization of Emotion on Social Media: Forms and Norms in Digital Mourning Practices." Social Media + Society 4 (1). https://doi.org/10.1177/2056305117744393.

Gilkison, Perrine, and Sydney Shep. 2012. "Mansfield as 'Man Alone?' Katherine Mansfield's Reading Experiences in Wartime Britain and France." The Journal of New Zealand Studies 13: 105-114.

Gill, Rosalind C. 2007. "Critical Respect: The Difficulties and Dilemmas of Agency and 'Choice' for Feminism: A Reply to Duits and van Zoonen." European Journal of Women's Studies 14 (1): 69-80. https://doi.org/10.1177/1350506807072318.

Gilmore, Lee, and Mark Van Proyen. 2005. AfterBurn : reflections on burning man. Albuquerque: University of New Mexico Press.

Giraud, Eva. 2015. "Subjectivity 2.0: Digital technologies, participatory media and communicative capitalism." Subjectivity 8 (2): 124-146.

https://doi.org/10.1057/sub.2015.5.

Glaser, April. 2018. "The Problem With \#DeleteFacebook." Slate. March 21, 2018. https://slate.com/technology/2018/03/dont-deletefacebook-thats-not-goodenough.html.

Goffman, Erving. 1969. The Presentation of Self in Everyday Life. London: Allen Lane. 
Golder, Ben. 2015. Foucault and the Politics of Rights. Stanford: Stanford University Press.

Goldman, Irene C. 1990. "Feminism, Deconstruction, and the Universal: A Case Study on 'Walden'." In Conversations: Contemporary Critical Theory and the Teaching of Literature, edited by Charles. Moran and Elizabeth F. Penfield, 120-131. Urbana: National Council of Teachers of English.

Google. 2018. "Digital Wellbeing." Google. Accessed 13 March, 2019. https://wellbeing.google/.

Gordhammer, Soren. 2009. Wisdom 2.0: Ancient Secrets for the Creative and Constantly Connected. New York: HarperCollins.

Gough, Brendan. 2003. "Deconstructing reflexivity." In Reflexivity: A Practical Guide for Researchers in Health and Social Sciences, edited by Linda Finlay and Brendan Gough, 21-36. Oxford: Blackwell Science.

Gough, Brendan. 2018. Contemporary Masculinities Embodiment, Emotion and Wellbeing. 1st ed. Cham: Springer International Publishing.

Grasz, Jennifer. 2015. "35 Percent of Employers Less Likely to Interview Applicants They Can't Find Online, According to Annual CareerBuilder Social Media Recruitment Survey." CareerBuilder. https://www.careerbuilder.ca/share/aboutus/pressreleasesdetail.aspx?sd=5\%2f14\%2 f2015\&siteid=cbpr\&sc_cmp1=cb_pr893_\&id=pr893\&ed=12\%2f31\%2f2015.

Gray, Colin M, Yubo Kou, Bryan Battles, Joseph Hoggatt, and Austin L. Toombs. 2018. "The Dark (Patterns) Side of UX Design." In Proceedings of the $2018 \mathrm{CH}$ Conference on Human Factors in Computing Systems, 1-14 Montreal: ACM. https://doi.org/10.1145/3173574.3174108.

Gray, Mary L. 2009. "Negotiating Identities/Queering Desires: Coming Out Online and the Remediation of the Coming-Out Story." Journal of Computer-Mediated Communication 14 (4): 1162-1189. https://doi.org/10.1111/j.10836101.2009.01485.x.

Green, Ben. 2019. The Smart Enough City: Putting Technology in Its Place to Reclaim Our Urban Future Cambridge, MA: MIT Press.

Greenfield, Adam. 2017. Radical Technologies: The Design of Everyday Life. London: Verso.

Gregg, Melissa. 2011. Work's intimacy. Cambridge, UK: Polity Press.

Gregg, Melissa. 2018. Counterproductive: Time Management in the Knowledge Economy. Durham: Duke University Press.

Griffey, Jason. 2012. Gadgets and Gizmos: Libraries and the Post PC Era (Library Technology Reports). Chicago: American Library Association. 
Grimes, Sara M. 2015. "Little Big Scene." Cultural Studies 29 (3): 379-400.

https://doi.org/10.1080/09502386.2014.937944.

Grush, Loren. 2018. "Elon Musk has removed Tesla and SpaceX's Facebook pages after Twitter challenge." The Verge. March 23, 2018.

https://www.theverge.com/2018/3/23/17156402/elon-musk-deleted-tesla-andspacex-facebook-pages-twitter-challenge.

Guyard, Carina, and Anne Kaun. 2018. "Workfulness: governing the disobedient brain." Journal of Cultural Economy 11 (6): 535-548.

https://doi.org/10.1080/17530350.2018.1481877.

Habermas, Jürgen. 1987. The Philosophical Discourse of Modernity. Cambridge, UK: Polity Press.

Haggart, Blayne. 2019. "Evaluating scholarship, or why I won't be teaching Shoshana Zuboff's The Age of Surveillance Capitalism." Blayne Haggart's Orangespace. February 15, 2019. https://blaynehaggart.com/2019/02/15/evaluating-scholarship-or-why-i-wontbe-teaching-shoshana-zuboffs-the-age-of-surveillance-capitalism/.

Hagood, Mack. 2019. Hush: Media and Sonic Self-Control. Durham: Duke University Press.

Han, Byung-Chul. 2017. Psychopolitics: Neoliberalism and New Technologies of Power. New York: Verso.

Hardt, Michael. 1999. "Affective Labor." boundary 26 (2): 89-100.

Hargittai, Eszter. 2007. "Whose space? Differences among users and non-users of social network sites by user background." Journal of Computer-Mediated Communication 13 (1): 276-279.

Hargittai, Eszter. 2012. "Open doors, closed spaces? Differentiated adoption of social network sites usage." In A Networked Self: Identity, Community and Culture on Social Network Sites, edited by Zizi Papacharissi, 146-168. New York: Routledge.

Harley, Aurora. 2015. "Ad Placement for Mobile." Nielsen Norman Group. July 5, 2015. https://www.nngroup.com/articles/ad-placement-mobile/.

Harris, Michael. 2017. Solitude: In Pursuit of a Singular Life in a Crowded World. London: Random House Books.

Harris, Tristan. 2016. "How Technology Hijacks People's Minds - from a Magician and Google's Design Ethicist." Observer. June 1, 2016. https://observer.com/2016/06/how-technology-hijacks-peoples-minds\%e2\%80\%8a\%e2\%80\%8afrom-a-magician-and-googles-design-ethicist/.

Hassan, Robert. 2019. Uncontained: Digital Disconnection and the Experience of Time. Melbourne: Grattan Street Press. 
Hawley, Josh. 2019. "We might be better off if Facebook, Instragram and Twitter vanished: Sen. John Hawley." USA Today. May 22, 2019.

https://www.usatoday.com/story/opinion/2019/05/22/facebook-instagram-twitterdo-more-harm-than-good-column/3751735002/.

Hearn, Alison. 2008. "'Meat, Mask, Burden`: Probing the contours of the branded 'self'." Journal of Consumer Culture 8 (2): 197-217. https://doi.org/10.1177/1469540508090086.

Hegel, George. (1807) 1977. Phenomenology of Spirit. Translated by A. V. Miller. Oxford: Oxford University Press.

Heidegger, Martin. (1927) 1953. Being and Time. Tubingen: Niemeyer.

Heidegger, Martin. 1977. The Question Concerning Technology and Other Essays. Translated by William Lovitt. New York: Garland.

Helmond, Anne. 2015. "The Platformization of the Web: Making Web Data Platform Ready." Social Media + Society 1 (2). https://doi.org/10.1177/2056305115603080.

Hepp, Andreas. 2016. "Pioneer communities: collective actors in deep mediatisation. Media, Culture \& Society 38(6). https://doi.org/10.1177/0163443716664484.

Herman, Edward S, and Noam Chomsky. 1988. Manufacturing Consent: The Political Economy of the Mass Media. New York: Pantheon Books.

Hesselberth, Pepita. 2017. "Discourses on disconnectivity and the right to disconnect." New Media \& Society 20 (5): 1994-2010. https://doi.org/10.1177/1461444817711449.

Hesselberth, Pepita. 2019. "The Grid as Structuring Paradox: A Case of Tiny Living." Soapbox 1 (2): 119-138.

Hetherington, Kevin. 1997. The Badlands of Modernity Heterotopia and Social ordering. London: Routledge.

Hjavard, Stig. 2008. "The Mediatization of Society: A Theory of the Media as Agents of Social and Cultural Change." Nordicom Review 29 (2): 105-134. https://doi.org/10.1515/nor2017-0181.

Hjavard, Stig. 2013. The Mediatisation of Culture and Society. Abingdon: Routledge.

Honig, Timothy J. 2017. "Heterotopia: A tool for understanding therapeutic space." Nordic Journal of Music Therapy 26 (1): 25-39.

https://doi.org/10.1080/08098131.2015.1095227.

Hook, Derek. 2007. Foucault, Psychology and the Analytics of Power. Houndmills: Palgrave Macmillan.

Houghton, Anne. 2013. Intentional Teaching: Promoting Purposeful Practice in Early Childhood Settings. Melbourne: Teaching Solutions. 
Huffington, Ariana. 2019. "Why Thrive Global Is Acquiring Boundless Mind." Thrive Global. October 16, 2019. https://thriveglobal.com/stories/arianna-huffington-boundlessmind-acquisition-neuroscience-artificial-intelligence/.

Hutchins, Brett. 2016. "'We don't need no stinking smartphones!' Live stadium sports events, mediatization, and the non-use of mobile media." Media, Culture \& Society 38 (3): 420436. https://doi.org/10.1177/0163443716635862.

Huws, Ursula. 2019. "The Hassle of Housework: Digitalisation and the Commodification of Domestic Labour." Feminist Review 123 (1): 8-23. https://doi.org/10.1177/0141778919879725.

ICO. 2019. Age appropriate design: a code of practice for online services. Information Commissioner's Office. May 31, 2019. https://ico.org.uk/about-the-ico/ico-andstakeholder-consultations/age-appropriate-design-a-code-of-practice-for-onlineservices/.

Idhe, Don. 2008. "The Designer's Fallacy and Technological Imagination." In Philosophy and Design: From Engineering to Architecture, edited by P. Kroes, P. E. Light Vermass, A. and S. A. Moore, 51-59. Dordrecht: Springer Netherlands.

Ingram, Matthew. 2018. "A new digital magazine forces you to unplug from the internet." Columbia Journalism Review, February 28, 2018. https://www.cjr.org/innovations/disconnect-magazine-only-works-offline.php.

Internet World Stats, 2020. "Internet World Stats: Usage and Population Statistics." https://www.internetworldstats.com/stats.htm

James, William. (1893) 1950. The principles of psychology. New York: Dover Publications.

Jameson, Fredric. 1984. "Postmodernism, or, the Cultural Logic of Late Capitalism." New Left Review 146: 53-92.

Jenkin, Matthew. 2015. "Tablets out, imagination in: the schools that shun technology." The Guardian. December 2, 2015. https://www.theguardian.com/teachernetwork/2015/dec/02/schools-that-ban-tablets-traditional-education-silicon-valleylondon.

Jiang, Shaohai, and Annabel Ngien. 2020. "The Effects of Instagram Use, Social Comparison, and Self-Esteem on Social Anxiety: A Survey Study in Singapore." Social Media + Society 6 (2). https://doi.org/10.1177/2056305120912488.

Johnson, Bobbie. 2010. "Privacy no longer a social norm, says Facebook founder." The Guardian. 11 January, 2010. https://www.theguardian.com/technology/2010/jan/11/facebook-privacy.

Johnson, Eric J, and Daniel Goldstein. 2003. "Do Defaults Save Lives?" Science 302 (5649): 1338-1339. https://doi.org/10.1126/science.1091721. 
Jones, Rhys, Jessica Pykett, and Mark Whitehead. 2011. "Changing behaviour in and Age of Libertarian Paternalism." Progress in Human Geography 35 (4): 483-501.

Jones, Rhys, Jessica Pykett, and Mark Whitehead. 2013. Changing behaviours on the rise of the psychological state. Cheltenham: Edward Elgar Publishing.

Jovicic, Suzana. Forthcoming. "Scrolling and the In-Between Spaces of Boredom: An Ethnographic Study of Youths on the Periphery of Vienna." Philosophy \& Technology.

Jurgenson, Nathan. 2012. "When Atoms Meet Bits: Social Media, the Mobile Web and Augmented Revolution." Future Internet 4: 83-91. https://doi.org/10.3390/fi4010083.

Jurgenson, Nathan. 2013. "The Disconnectionists." The New Inquiry. November 13, 2013. https://thenewinquiry.com/the-disconnectionists/.

Jurgenson, Nathan. 2016. "Fear of Screens." The New Inquiry. January 25, 2016.

https://thenewinquiry.com/fear-of-screens/.

Jurgenson, Nathan. 2019. The Social Photo: On Photography and Social Media. London: Verso.

Kahneman, Daniel. 2011. Thinking, Fast and Slow. New York: Farrar, Straus and Giroux.

Karppi, Tero, and David B. Nieborg. 2020. "Facebook confessions: Corporate abdication and Silicon Valley dystopianism." New Media \& Society. https://doi.org/10.1177/1461444820933549.

Karppi, Tero. 2011. "Digital Suicide and the Biopolitics of Leaving Facebook." Transformations 20: 1-28.

Karppi, Tero. 2018. Disconnect: Facebook's Affective Bonds. Minneapolis: University of Minnesota Press.

Kaun, Anne, and Christian Schwarzenegger. 2014. "'No media, less life?" Online disconnection in mediatized worlds." First Monday 19 (11).

https://firstmonday.org/ojs/index.php/fm/article/view/5497.

Kelty, Christopher M. 2009. Two Bits: The Cultural Significance of Free Software. London: Duke University Press.

Kessler, Sandra, D J Ashenden, Raewyn W. Connell, and Gary W. Dowsett. 1982. Ockers and Disco-maniacs. Sydney: Inner City Education Center.

Kondō, Marie. 2014. The Life-Changing Magic of Tidying Up: The Japanese Art of Decluttering and Organizing. Berkeley: Ten Speed Press.

Kozinets, Robert, V. 2002. "Can Consumers Escape the Market? Emancipatory Illuminations from Burning Man." Journal of Consumer Research 29 (1): 20-38.

https://doi.org/10.1086/339919 
Krakauer, Jon. 1997. Into the Wild. New York: Anchor Books.

Krieg, Richard. 1995. "Information technology and low-income, inner city communities. Journal of Urban Technology." Journal of Urban Technology 3 (1): 1-17.

Kroese, Floor M, David R. Marchiori, and Denise T. D. de Ridder. 2015. "Nudging healthy food choices: a field experiment at the train station." Journal of Public Health 38 (2): 133137. https://doi.org/10.1093/pubmed/fdv096.

KTVU. 2015. "No tech signs appear in city parks" Fox News. http://www.ivan.cash/no-techzone.

Kuehn, Kathleen, and Michael Daubs. 2016. "The Holy Trail: Rethinking 'Value' in Google's Ubiquitous Mapping Project." MediaNZ16 (1): 1-16.

http://dx.doi.org/10.11157/medianz-vol16iss1id199.

Kuehn, Kathleen, and Thomas F Corrigan. 2013. "Hope Labor: The Role of Employment Prospects in Online Social Production." 1 (1). http://www.polecom.org/index.php/polecom/article/view/9.

Kuiken, Jeffrey, Anne Schuth, Martijn Spitters, and Maarten Marx. 2017. "Effective Headlines of Newspaper Articles in a Digital Environment." Digital Journalism 5 (10): 1300-1314. https://doi.org/10.1080/21670811.2017.1279978.

Kuntsman, Adi, and Esperanza Miyake. 2019. "The paradox and continuum of digital disengagement: denaturalising digital sociality and technological connectivity." Media, Culture \& Society 41 (6): 901-913 https://doi.org/10.1177/0163443719853732.

Lacey, Cherie, Catherine Caudwell, and Alex Beattie. 2019. "The Perfect User." Real Life. September 5, 2019. https://reallifemag.com/the-perfect-user/.

Lachney, Michael, and Taylor Dotson. 2018. "Epistemological Luddism: Reinvigorating a Concept for Action in 21st Century Sociotechnical Struggles." Social Epistemology 32 (4): 228-240. https://doi.org/10.1080/02691728.2018.1476603.

Lanier, Jaron. 2018. Ten Arguments For Deleting Your Social Media Accounts Right Now. London: The Bodley Head.

Lasch, Christopher. 1979. Culture of Narcissism: American Life in an Age of Diminishing Expectation. New York: Warner Books.

Laterza, Vito. 2018. "Cambridge Analytica, independent research and the national interest." Anthropology Today 34 (3): 1-2. https://doi.org/10.1111/1467-8322.12430.

Latour, Bruno. 2005. Reassembling the Social: An Introduction to Actor-Network-Theory. Oxford: Oxford University Press.

Lazzarato, Maurizio. 2006. "The concepts of life and the living in the societies of control." In Deleuze and the Social, edited by Martin Fuglsang and Bent Meier Sørensen, 171-190. Edinburgh: Edinburgh University Press. 
Leary, Timothy. 1999. Turn On, Tune In, Drop Out. 6th ed. Berkeley: Ronin Publishing.

Lee, Angela M, Seth C. Lewis, and Matthew Powers. 2014. "Audience Clicks and News Placement: A Study of Time-Lagged Influence in Online Journalism." Communication Research 41 (4): 505-530. https://doi.org/10.1177/0093650212467031.

Lee, John A. 1964. Shiner Slattery. Auckland: Collins.

Lee, Uichin, Subin Yang, Minsam Ko, and Joonwon Lee. 2014. "Supporting temporary non-use of smartphones." In Proceedings of the CHI'74 Conference Refusing, Limiting, Departing: Why We Should Study Technology Non-Use, New York: ACM.

Lee-Won, Roselyn J, Leo Herzog, and Sung Gwan Park. 2015. "Hooked on Facebook: The Role of Social Anxiety and Need for Social Assurance in Problematic Use of Facebook." Cyberpsychology, Behavior, and Social Networking 18 (10): 567-574. https://doi.org/10.1089/cyber.2015.0002.

Leslie, Ian. 2016. "The Scientists Who Make Apps Addictive." 1843: The Economist. October 20, 2016. https://www. 1843magazine.com/features/the-scientists-who-make-appsaddictive.

Levina, Marina, and Amy Adele Hasinoff. 2016. "The Silicon Valley Ethos: Tech Industry Products, Discourses, and Practices." Television \& New Media 18 (6): 489-495. https://doi.org/10.1177/1527476416680454.

Levy, David M. 2016. Mindful Tech: How to Bring Balance to Our Digital Lives. New Haven: Yale University Press.

Lewis, Clayton, Peter Polson, John Rieman, and Cathleen Wharton. 1990. "Testing a walkthrough methodology for theory-based design of walk-up-and-use interfaces." In Proceedings of the SIGCHI conference on human factors in computing systems, 235242, Seattle: ACM. https://doi.org/10.1145/97243.97279.

Lewis, Justin, and Stephen Cushion. 2009. "The Thirst to be First." Journalism Practice 3 (3): 304-318. https://doi.org/10.1080/17512780902798737.

Lewis, Paul. 2017. "'Our minds can be hijacked': the tech insiders who fear a smartphone dystopia." The Guardian. October 5, 2017.

https://www.theguardian.com/technology/2017/oct/05/smartphone-addictionsilicon-valley-dystopia.

Lewis, Randall, Justin M Rao, and David H Reiley. 2015. "Measuring the Effects of Advertising: The Digital Frontier." In Economic Analysis of the Digital Economy, edited by Avi Goldfarb, Shane M Greenstein and Catherine E Tucker, 191-218. Chicago: University of Chicago Press.

Light, Ben, and Elija Cassidy. 2014. "Strategies for the suspension and prevention of connection: Rendering disconnection as socioeconomic lubricant with Facebook." New Media \& Society 16 (7): 1169-1184. https://doi.org/10.1177/1461444814544002. 
Light, Ben, Jean Burgess, and Stefanie Duguay. 2016. "The walkthrough method: An approach to the study of apps." New Media \& Society 20 (3): 881-900.

https://doi.org/10.1177/1461444816675438.

Light, Ben. 2014. Disconnecting with Social Networking Sites. Houndmills: Palgrave MacMillan.

Lilja, Mona, and Stellan Vinthagen. 2014. "Sovereign power, disciplinary power and biopower: resisting what power with what resistance?" Journal of Political Power 7 (1): 107-126. https://doi.org/10.1080/2158379X.2014.889403.

Lilspace. 2019. "Getlilspace." Lilspace. Accessed 21 July, 2020. http://www.getlilspace.com/.

Lindstrom, Martin. 2011. Brandwashed: Tricks Companies Use to Manipulate Our Minds and Persuade Us to Buy. New York: Crown Business.

Ling, Rich. 2008. New Tech, New Ties: How Mobile Communication is Reshaping Social Cohesion. Cambridge, MA: MIT Press.

Ling, Rich. 2016. "Soft coercion: Reciprocal expectations of availability in the use of mobile communication." First Monday 21 (9). https://firstmonday.org/ojs/index.php/fm/article/view/6814.

Liu, Ziming. 2005. "Reading behavior in the digital environment: Changes in reading behavior over the past ten years." Journal of Documentation 61 (6): 700-712. https://doi.org/doi:10.1108/00220410510632040.

Lorey, Isabell. 2006. "Governmentality and Self-Precarization: On the normalization of cultural producers." Translated by Lisa Rosenblatt and Dagmar Fink, Machines and Subjectivation. https://transversal.at/pdf/journal-text/462/

Lovink, Geert. 2019. Sad By Design: On Platform Nihilism. London: Pluto Press.

Lucero, Leanna. 2017. "Safe spaces in online places: social media and LGBTQ youth." Multicultural Education Review 9 (2): 117-128. https://doi.org/10.1080/2005615X.2017.1313482.

Lukes, Steven. 2005. Power: A Radical View. 2nd ed. Houndmills: Palgrave Macmillan.

Lupton, Deborah. 2014. Digital Sociology. Abingdon: Routledge.

Lyngs, Ulrik, Kai Lukoff, Petr Slovak, Reuben Binns, Adam Slack, Michael Inzlicht, Max Van Kleek, and Nigel Shadbolt. 2019. "Self-Control in Cyberspace: Applying Dual Systems Theory to a Review of Digital Self-Control Tools." In Proceedings of the $2019 \mathrm{CHI}$ Conference on Human Factors in Computing Systems, 1-18, Glasgow: ACM. https://doi.org/10.1145/3290605.3300361.

Lyngs, Ulrik, Kai Lukoff, Petr Slovak, William Seymour, Helena Webb, Marina Jirotka, Jun Zhao, Max Van Kleek, and Nigel Shadbolt. 2020. "'I Just Want to Hack Myself to Not Get Distracted": Evaluating Design Interventions for Self-Control on Facebook." In 
Proceedings of the $2020 \mathrm{CH} /$ Conference on Human Factors in Computing Systems, 1-18, Honolulu: ACM. https://doi.org/10.1145/3313831.3376672.

Mackenzie, Adrian. 2013. "Programming subjects in the regime of anticipation: Software studies and subjectivity." Subjectivity 6 (4): 391-405.

https://doi.org/10.1057/sub.2013.12.

Macleod, Gordon, and Kevin Ward. 2002. "Spaces of utopia and dystopia: landscaping the contemporary city." Geografiska Annaler: Series B, Human Geography 84 (3-4): 153170. https://doi.org/10.1111/j.0435-3684.2002.00121.x.

Mahatody, Thomas, Mouldi Sagar, and Christophe Kolski. 2010. "State of the Art on the Cognitive Walkthrough Method, Its Variants and Evolutions." International Journal of Human-Computer Interaction 26 (8): 741-785. https://doi.org/10.1080/10447311003781409.

Mainwaring, Scott D., Michele F. Chang, and Ken Anderson. 2004. "Infrastructures and Their Discontents: Implications for Ubicomp." In: Ubiqutious Computing. UbiComp 2004. Lecture Notes in Computer Science, edited by N. Davies, E. D. Mynatt and I. Siio, 3205: 418-432. Berlin: Springer. https://doi.org/10.1007/978-3-540-30119-6_25.

Malle, Bertram F., and Joshua Knobe. 1997. "The Folk Concept of Intentionality." Journal of Experimental Social Psychology 33 (2): 101-121. https://doi.org/10.1006/jesp.1996.1314.

Mannell, Kate. 2018. "A typology of mobile messaging's disconnective affordances." Mobile Media \& Communication 7 (1): 76-93. https://doi.org/10.1177/2050157918772864.

Marino, Sara. 2015. "Making Space, Making Place: Digital Togetherness and the Redefinition of Migrant Identities Online." Social Media + Society 1 (2):

https://doi.org/10.1177/2056305115622479.

Mark, Gloria, Daniela Gudith, and Ulrich Klocke. 2008. "The Cost of Interrupted Work: More Speed and Stress." In Proceedings of the SIGCHI Conference on Human Factors in Computing Systems, 107-110. New Yorl: ACM. https://dl.acm.org/doi/10.1145/1357054.1357072.

Martin, Garry L, and Joseph Pear. 2010. Behavior Modification: What It Is and How To Do It. 9th ed. Cambridge, UK: Pearson.

Marwick, Alice. 2015. "Instafame: Luxury Selfies in the Attention Economy." Public Culture 27 (1 (75)): 137-160. https://doi.org/10.1215/08992363-2798379.

Mayton, Joseph. 2015. "'No Tech Zone' sign in San Francisco befuddles residents." The Guardian. September 22, 2015. https://www.theguardian.com/usnews/2015/sep/22/no-tech-zone-sign-san-francisco-alamo-square.

Mazmanian, Melissa. 2013. "Avoiding the Trap of Constant Connectivity: When Congruent Frames Allow for Heterogeneous Practices." Academy of Management Journal 56 (5): 1225-1250. https://doi.org/10.5465/amj.2010.0787. 
McCormick, E. H. 1959. New Zealand Literature: A Survey. Oxford University Press: London.

McCullough, Malcolm. 2015. Ambient Commons: Attention in the Age of Embodied Information. Cambridge, MA: MIT Press.

McDaniel, Brandon T, and Sarah M. Coyne. 2016. "Technology interference in the parenting of young children: Implications for mothers' perceptions of coparenting." The Social Science Journa/53 (4): 435-443. https://doi.org/10.1016/j.soscij.2016.04.010.

McGonigal, Jane. 2011. Reality Is Broken: Why Games Make Us Better and How They Can Change the World. London: Penguin Books.

McGoogan, Cara. 2016. "German city installs traffic lights in pavements to protect texting pedestrians." The Telegraph. April 26, 2016.

https://www.telegraph.co.uk/technology/2016/04/26/german-city-installs-trafficlights-in-pavements-to-protect-text/

McGuigan, Jim. 2009. Coo/ Capitalism. New York: Pluto Press.

McKay, Jenny. 2019. The Magazines Handbook. 3rd ed. Abingdon: Routledge.

McNamee, Roger. 2019. Zucked: Waking Up to the Facebook Catastrophe. New York: HarperCollins.

McNay, Lois. 1992. Foucault and Feminism: Power, Gender and the Self. Cambridge: Polity Press.

McNay, Lois. 2000. Gender and Agency: Reconfiguring the Subject in Feminist and Social Theory. Cambridge: Polity Press.

Meissner, Miriam. 2019. "Against accumulation: lifestyle minimalism, de-growth and the present post-ecological condition." Journal of Cultural Economy 12 (3): 185-200. https://doi.org/10.1080/17530350.2019.1570962.

Mejias, Ulises Ali. 2013. Off the Network: Disrupting the Digital World. Minneapolis: University of Minnesota Press.

Merlino, James, 2019, "Mobile Phones To Be Banned Next Year In All State Schools," Office of the Victoria Premier, June 26, 2019. https://www.premier.vic.gov.au/mobile-phonesto-be-banned-next-year-in-all-state-schools/.

Messerschmidt, James W. 2018. Hegemonic Masculinity: Formulation, Reformulation, and Amplification. Lanham: Rowman \& Littlefield.

Mewburn, Inger, and Pat Thomson. 2017. "Towards an Academic Self? Blogging During the Doctorate." In The Digital Academic: Critical Perspectives on Digital Technologies in Higher Education, edited by Deborah Lupton, Inger Mewburn and Pat Thomson, 20-36. London: Routledge. 
Meyrowitz, Joshua. 1985. No Sense of Place: The Impact of the Electronic Media on Social Behavior. Oxford: Oxford University Press.

Miller, Jean Baker. 2008. "VI. Connections, Disconnections, and Violations." Feminism \& Psychology 18 (3): 368-380. https://doi.org/10.1177/0959353508092090.

Miller, Paul. 2013. "I'm still here: back online after a year without the internet." The Verge. May 1, 2013. https://www.theverge.com/2013/5/1/4279674/im-still-here-back-onlineafter-a-year-without-the-internet.

Miller, Peter, and Nikolas Rose. 2008. Governing the Present: Administering Economic, Social and Personal Life. Cambridge, UK: Polity Press.

Mitesser, Steph. 2018. "You Can't Just Tell Everyone to Leave Facebook: Tech is Not a Problem of Individual Consumption." The Outline. April 3, 2018. https://theoutline.com/post/4040/you-cant-just-tell-everyone-to-leave-facebook.

More, Thomas (1516) 2020. "Utopia." In The Essential Works of Thomas More, edited by Gerard B. Wegemer and Stephen W. Smith, 141-216. New Haven: Yale University Press.

Morozov, Evgeny. 2011. The Net Delusion: The Dark Side of Internet Freedom. New York: Public Affairs.

Morozov, Evgeny. 2014. To Save Everything, Click Here: Technology, Solutionism and the Urge to Fix Problems that Don't Exist. London: Penguin Books.

Morozov, Evgeny. 2019. "Capitalism's New Clothes: Shoshana Zuboff's new book on "surveillance capitalism" emphasizes the former at the expense of the latter." The Baffler. February 4, 2019. https://thebaffler.com/latest/capitalisms-new-clothesmorozov.

Mosco, Vincent. 2019. The Smart City in a Digital World. Bingley: Emerald Publishing Limited.

Moylan, Tom, and Raffaella Baccolini, eds. 2013. Dark Horizons: Science Fiction and the Dystopian Imagination. New York: Routledge.

Mozilla. 2020. "Mozilla makes browsers, apps, code and tools that put people before profit." Mozilla. Accessed 21 July, 2020. https://www.mozilla.org/en-US/about/.

Mozilla. n.d. "Online and offline events." Mozilla. Accessed 29 October 2018. https://developer.mozilla.org/enUS/docs/Web/API/NavigatorOnLine/Online_and_offline_events.

Mulgan, John. (1939) 2010. Man Alone. Auckland: Penguin Books.

Munn, Luke. 2020. Logic of Feeling: Technology's Quest to Capitalize Emotion. Lanham: Rowman \& Littlefield. 
Murdock, Graham, and Peter Golding. 2004. "Dismantling the digital divide: Rethinking the dynamics of participation and exclusion." In Toward a Political Economy of Culture. Capitalism and Communication in the Twenty-first Century, edited by Colin Sparks and Andrew Calabrese, 244-260. Lanham: Rowman \& Littlefield.

Myerson, Joel, ed. 2000. Transcendentalism: A Reader. Oxford: Oxford University Press.

Natale, Simone, and Emiliano Treré. 2020. "Vinyl won't save us: reframing disconnection as engagement." Media, Culture \& Society. 42 (4): 626-633.

https://doi.org/10.1177/0163443720914027.

Neuman, W. Russell. 2016. The Digital Difference: Media Technology and the Theory of Communication Effects. Cambridge, MA: Harvard University Press.

Neves, Barbara Barbosa, and Geoffrey; Mead. 2018. "The interpretative and ideal-type approach: rethinking digital non-use(s) in a Weberian perspective." In Theorizing Digital Divides, edited by Massimo Ragnedda and Glenn W. Muschert, 48-60. Milton Park, Abingdon: Routledge.

Newport, Carl. 2019. Digital Minimalism: Choosing a Focused Life in a Noisy World. London: Portfolio.

Nicas, Jack. 2019. "Apple Cracks Down on Apps That Fight iPhone Addiction." The New York Times. April 27, 2019. https://www.nytimes.com/2019/04/27/technology/applescreen-time-trackers.html.

Nieborg, David B, and Thomas Poell. 2018. "The platformization of cultural production: Theorizing the contingent cultural commodity." New Media \& Society 20 (11): 42754292. https://doi.org/10.1177/1461444818769694.

Nixon, Sean. 1997. "Exhibiting Masculinity." In Representation: Cultural Representations and Signifying Practices, edited by Stuart Hall, 291-336. Walton Hall: The Open University.

Norman, Donald A. 2004. Emotional Design: Why We Love (or Hate) Everyday Things. New York: Basic Books.

Norris, Pippa. 2001. Digital Divide: Civic Engagement, Information Poverty, and the Internet Worldwide. Cambridge, UK: Cambridge University Press.

Odell, Jenny. 2019. How to do Nothing: Resisting the Attention Economy. Brooklyn: Melville House.

OECD. 2001. Understanding the Digital Divide, edited by the Organisation for Economic Cooperation and Development. Paris: OECD.

Ofcom. 2016. "Communications Market Report 2016." Ofcom. August 4, 2016. https://www.ofcom.org.uk/_data/assets/pdf_file/0024/26826/cmr_uk_2016.pdf.

Ohtomo, Shoji. 2013. "Effects of habit on intentional and reactive motivations for unhealthy eating." Appetite 68: 69-75. https://doi.org/10.1016/j.appet.2013.04.014. 
Oliffe, John L, Steve Robertson, Mary T. Kelly, Philippe Roy, and John S. Ogrodniczuk. 2010. "Connecting Masculinity and Depression Among International Male University Students." Qualitative Health Research 20 (7): 987-998. https://doi.org/10.1177/1049732310365700.

Olson, Penny. 2013. We Are Anonymous: Inside the Hacker World of LulzSec, Anonymous, and the Global Cyber Insurgency New York: Back Bay Books.

Orben, Amy, and Andrew K. Przybylski. 2019. "The association between adolescent well-being and digital technology use." Nature Human Behaviour3 (2): 173-182. https://doi.org/10.1038/s41562-018-0506-1.

Orben, Amy, Tobias Dienlin, and Andrew K. Przybylski. 2019. "Social media's enduring effect on adolescent life satisfaction." Proceedings of the National Academy of Sciences: 116 (21): 10226-10228. https://doi.org/10.1073/pnas.1902058116.

Orchard, Rob, and Marcus Webb. 2018. "Slow Journalism matters." Delayed Gratification 31, April 2018.

Ouellette, Laurie, and Jacquelyn Arcy. 2015. "'Live Through This': Feminist Care of the Self 2.0." Frame 28 (2): 95-114.

Owler, Kathryn. 2010. "A 'problem' to be managed?: Completing a PhD in the Arts and Humanities." Arts and Humanities in Higher Education 9 (3): 289-304. https://doi.org/10.1177/1474022209356330.

Paasonen, Susanna. 2016. "Fickle focus: Distraction, affect and the production of value in social media." First Monday 21 (10). https://firstmonday.org/ojs/index.php/fm/article/view/6949.

Paasonen, Susanna. 2018. "Affect, data, manipulation and price in social media." Distinktion: Journal of Social Theory 19 (2): 214-229. https://doi.org/10.1080/1600910X.2018.1475289.

Papacharissi, Zizi. 2010. "Privacy as a luxury commodity." First Monday 15 (8). https://firstmonday.org/ojs/index.php/fm/article/view/3075.

Pardes, Arielle. 2018. "These Magical Sunglasses Block All the Screens Around You." Wired. July 10, 2018. https://www.wired.com/story/irl-glasses-screen-blocking/.

Paris, Cody Morris, Edward Alexander Berger, Simon Rubin, and Mallory Casson. 2015. "Disconnected and Unplugged: Experiences of Technology Induced Anxieties and Tensions While Traveling." In: Information and Communication Technologies in Tourism 2015: Proceedings of the International Conference in Lugano, Switzerland, February 3-6, edited by lis Tussyadiah and Alessandro Inversini. Lugano: Springer.

Pariser, Eli. 2011. The Filter Bubble: What the Internet is Hiding from You. London: Penguin Books. 
Parkin, Simon. 2019. "Has dopamine got us hooked on tech?" The Guardian. March 4, 2019. https://www.theguardian.com/technology/2018/mar/04/has-dopamine-got-ushooked-on-tech-facebook-apps-addiction.

Pedwell, Carolyn. 2017a. "Habit and the Politics of Social Change: A Comparison of Nudge Theory and Pragmatist Philosophy." Body \& Society 23 (4): 59-94. https://doi.org/10.1177/1357034X17734619.

Pedwell, Carolyn. 2017b. "Transforming habit: revolution, routine and social change." Cultural Studies 31 (1): 93-120. https://doi.org/10.1080/09502386.2016.1206134.

Perry, Grayson. 2017. The Descent of Man. London: Penguin Books.

Peters, John Durham. 2015. The Marvelous Clouds: Toward a Philosophy of Elemental Media. Chicago: The University of Chicago Press.

Peters, Michael, A, and Walter Humes. 2003. "Educational Futures: utopias and heterotopias." Policy Futures in Education 1 (3): 428-439.

Plantin, Jean-Christophe, Carl Lagoze, Paul N. Edwards, and Christian Sandvig. 2016. "Infrastructure studies meet platform studies in the age of Google and Facebook." New Media \& Society 20 (1): 293-310. https://doi.org/10.1177/1461444816661553.

Plath, James. 1999. "Shadow Rider: The Hemingway Hero as Western Archetype." In Hemingway and the natural world, edited by Robert E Fleming, 69-86. Moscow, Idaho: University of Idaho Press.

Plato. (340 BCE) 2005. Phaedrus. Penguin Classics.

Plaut, Ethan. 2014. "Commitments not to Communicate Before and after Digital Media: A Study of the Will and Ways to Disconnect Under Changing Conditions." PhD diss., Stanford University.

Plaut, Ethan. 2015. "Technologies of avoidance: The swear jar and the cell phone." First Monday 20 (11) https://www.firstmonday.org/ojs/index.php/fm/article/view/6295.

Portwood-Stacer, Laura. 2012a. "How We Talk About Media Refusal, Part 3: Aesthetics." Flow Journal. October 14, 2012. https://www.flowjournal.org/2012/10/how-we-talk-aboutmedia-refusal-part-3-aesthetics/.

Portwood-Stacer, Laura. 2012b. "How We Talk About Social Media Refusal Part 2: Asceticism." Flow Journal. September 10, 2012. http://www.flowjournal.org/2012/09/media-refusal-part-2-asceticism/.

Portwood-Stacer, Laura. 2012c. "Media refusal and conspicuous non-consumption: The performative and political dimensions of Facebook abstention." New Media \& Society 15 (7): 1041-1057. https://doi.org/10.1177/1461444812465139. 
Portwood-Stacer, Laura. 2014. "Care Work and the Stakes of Social Media Refusal." New Criticals. http://www.newcriticals.com/care-work-and-the-stakes-of-social-mediarefusal/prin.

Power, Matthew. 2012. "The Cult of Chris McCandless." Men's Journal. October 15, 2012. https://www.mensjournal.com/features/the-cult-of-chris-mccandless-20121015/.

Pruchniewska, Urszula. 2019. "'A group that's just women for women": Feminist affordances of private Facebook groups for professionals." New Media \& Society 21 (6): 13621379. https://doi.org/10.1177/14461444818822490.

Qu, Sandy, and John Dumay. 2011. "The Qualitative Research Interview." Qualitative Research in Accounting and Management 8 (3): 238-264.

Raley, Rita. 2013. "Dataveillance and countervailance." In 'Raw Data' is an Oxymoron, edited by Lisa Gitelman, 121-146. Cambridge, MA: MIT Press.

Ransomly. 2020. "Ransomly." Accessed 21 July, 2020. https://www.ransomly.com/.

Rauch, Jennifer. 2018. Slow Media: Why "Slow" is Satisfying, Sustainable, and Smart. New York: Oxford University Press.

Reer, Felix, Wai Yen Tang, and Thorsten Quandt. 2019. "Psychosocial well-being and social media engagement: The mediating roles of social comparison orientation and fear of missing out." New Media \& Society 21 (7): 1486-1505.

https://doi.org/10.1177/1461444818823719.

Reinecke, Leonard, Stefan Aufenanger, Manfred E. Beutel, Michael Dreier, Oliver Quiring, Birgit Stark, Klaus Wölfling, and Kai W. Müller. 2017. "Digital Stress over the Life Span: The Effects of Communication Load and Internet Multitasking on Perceived Stress and Psychological Health Impairments in a German Probability Sample." Media Psychology 20 (1): 90-115. https://doi.org/10.1080/15213269.2015.1121832.

Rheingold, Howard. 2012. Net Smart: How to Thrive Online. Cambridge, MA: MIT Press.

Richards, Neil. 2008. "Intellectual Privacy." Yale Law Journa/87 (2): 387-446.

Rider, Karina, and David Murakami Wood. 2018. "Condemned to connection? Network communitarianism in Mark Zuckerberg's "Facebook Manifesto"." New Media \& Society 21 (3): 639-654. https://doi.org/10.1177/1461444818804772.

Ritzer, George, and Nathan Jurgenson. 2010. "Production, Consumption, Prosumption: The nature of capitalism in the age of the digital 'prosumer'." Journal of Consumer Culture 10 (1): 13-36. https://doi.org/10.1177/1469540509354673.

Roberts, Jessica, and Michael Koliska. 2014. "The effects of ambient media: What unplugging reveals about being plugged in." First Monday 19 (8). https://firstmonday.org/ojs/index.php/fm/article/view/5220. 
Rodino-Colocino, Michelle. 2006. "Laboring under the digital divide." New Media \& Society 8 (3): 487-511. https://doi.org/10.1177/1461444806064487.

Rogers, Richard. 2013. Digital Methods. Cambridge, MA: MIT Press.

Rose, Nikolas. 1998. Inventing Our Selves: Psychology, Power, and Personhood. Cambridge, UK: Cambridge University Press.

Rose, Nikolas. 1999. Governing the Soul: Shaping of the Private Self. 2nd ed. London: Free Association Books.

Rosenberg, Hananel. 2019. "The "flashpacker" and the "unplugger": Cell phone (dis)connection and the backpacking experience." Mobile Media \& Communication 7 (1): 111-130. https://doi.org/10.1177/2050157918777778.

Rossiter, Ned. 2004. "Creative Industries, Comparative Media Theory and the Limits of Critique from Within." TOPIA: Canadian Journal of Cultural Studies 11: 21-28.

Rouvroy, Antoinette. 2013. "The end(s) of critique : Data behaviourism versus due process." In Privacy, Due Process and the Computational Turn: The Philosophy of Law Meets the Philosophy of Technology, edited by Mireille Hildebrandt and Katja De Vries, 143-167. London: Taylor \& Francis.

Royal Society for Public Health. 2018. "Scroll Free September." Royal Society for Public Health. May 10, 2019. https://www.rsph.org.uk/our-work/campaigns/scroll-freeseptember.html.

Russell, Amy. 2009. "Lesbians Surviving Culture: Relational-Cultural Theory Applied to Lesbian Connection." Affilia: Journal of Women and Social Work 24 (4): 406-416.

Rymarczuk, Robin, and Maarten Derksen. 2014. "Different spaces: Exploring Facebook as heterotopia." First Monday 19 (6).

https://firstmonday.org/ojs/index.php/fm/article/view/5006.

Satchell, Christine, and Paul Dourish. 2009. "Beyond the user: use and non-use in HCl." In Proceedings of the 27st Annual Conference of the Australian Computer-Human Interaction Special Interest Group: Design: Open 24/7, 9-16 Melbourne: ACM. https://doi.org/10.1145/1738826.1738829.

Schippers, Mimi. 2007. "Recovering the feminine other: masculinity, femininity, and gender hegemony." Theory and Society 36 (1): 85-102. https://doi.org/10.1007/s11186-0079022-4.

Schirato, Tony, Angi Buettner, Thierry Jutel, and Geoff Stahl. 2010. Understanding media studies. South Melbourne: Oxford University Press.

Schüll, Natasha Dow. 2012. Addiction by Design: Machine Gambling in Las Vegas. Cambridge, MA: MIT Press. 
Schüll, Natasha Dow. 2016. "Data for life: Wearable technology and the design of self-care." BioSocieties 11 (3): 317-333.

Schultz, Dan. 2017. "Internet Noise." Accessed 21 March, 2018. http://makeinternetnoise.com/index.html.

Seaver, Nick. 2019. "Captivating algorithms: Recommender systems as traps." Journal of Material Culture 24 (4): 421-436. https://doi.org/10.1177/1359183518820366.

Selwyn, Neil. 2003. "Apart from technology: understanding people's non-use of information and communication technologies in everyday life." Technology in Society 25 (1): 99116. https://doi.org/10.1016/S0160-791X(02)00062-3.

Selwyn, Neil. 2004. "Reconsidering Political and Popular Understandings of the Digital Divide." New Media \& Society 6 (3): 341-362. https://doi.org/10.1177/1461444804042519.

Selwyn, Neil. 2006. "Digital division or digital decision? A study of non-users and low-users of computers." Poetics 34 (4-5): 273-292. https://doi.org/10.1016/j.poetic.2006.05.003.

Serazio, Michael. 2020. "How news went guerrilla marketing: a history, logic, and critique of brand journalism." Media, Culture \& Society. https://doi.org/10.1177/0163443720939489.

Servon, Lisa. 2002. Bridging the Digital Divide: Technology, Community, and Public Policy. Malden: Blackwell.

Shamir, Milette, and Jennifer Travis. 2002. "Introduction." In Boys don't cry? : rethinking narratives of masculinity and emotion in the U.S, edited by Milette Shamir and Jennifer Travis, 1-21. New York: Columbia University Press.

Shamonsky, Dorothy. 2018. "Developing a Code of Ethics for UX Design: What We Can Learn from the Field of Architecture." User Experience. October, 2018.

https://uxpamagazine.org/developing-a-code-of-ethics/.

Shenker, Barry. 2010. Intentional Communities: Ideology and Alienation in Communal Societies. Boston: Routledge.

Shepherd, Robin-Marie, and Robert J. Edelmann. 2005. "Reasons for internet use and social anxiety." Personality and Individual Differences 39 (5): 949-958. https://doi.org/10.1016/j.paid.2005.04.001.

Shieber, Jonathan. 2017. "Dopamine Labs slings tools to boost and reduce app addiction." Tech Crunch. February 13, 2017. https://techcrunch.com/2017/02/13/dopaminelabs-slings-tools-to-boost-and-reduce-app-addiction/.

Shoemaker, Karl. 2011. Sanctuary and crime in the middle ages, 400-1500. New York: Fordham University Press. 
Shun-hing, Chan. 2009. "Politics of Female Subjectivities and the Everyday: The Case of the Hong Kong Feminist Journal Nuliu." Feminist Review92 (1): 36-53.

https://doi.org/10.1057/fr.2009.7.

Sieberg, Daniel. 2011. The Digital Diet: The 4-step Plan to Break Your Tech Addiction and Regain Balance in Your Life. New York: Harmony.

Siegert, Bernhard. 2015. Cultural Techniques: Grids, Filters, Doors, and Other Articulations of the Real. New York: Fordham University Press.

Siempo. 2019. "Welcome to Siempo." YouTube. April 10, 2019.

https://www.youtube.com/watch?v=eNlj32aPasA.

Silverman, Jacob. 2015. Terms of Service: Social Media and the Price of Constant Connection. New York: Harper Collins.

Skinner, B. F. 1948. Walden Two. Indianapolis: Hackett Publishing Company.

Smith, Matthew, and Daniel Keane. 2019. "Hannah Gadsby to use phone pouches to stop people filming her shows". ABC Radio Adelaide. 26 March, 2018.

https://www.abc.net.au/news/2019-03-26/hannah-gadsby-uses-phone-lockingtechnology-for-latest-tour/10939478.

Smith, Noah (@Noahpinion). 2017. "15 years ago, the internet was an escape from the real world. Now, the real world is an escape from the internet." Twitter. April 29, 2017. https://twitter.com/Noahpinion/status/902301308702515202.

Smith, Peter A, and Alicia D Sanchez. 2015. "Let's play, video streams, and the evolution of new digital literacy." In Learning and Collaboration Technologies, edited by Panayiotis Zaphiris and Andri loannou, 520-527. Los Angeles: Springer.

Social Media Addiction Reduction Technology Act of 2019. S.Res. $19429,116^{\text {th }}$ Cong. (2019).

Sohn, Heidi. 2008. "Heterotopia: anamnesis of a medical term." In Heterotopia and the City: Public Space in a Postcivil Society, edited by Michiel Dehaene and Lieven De Cauter, 41-50. Abingdon: Routledge.

Soja, Edward. 1996. Thirdspace: Journeys to Los Angeles and Other Real-and-Imagined Places. London: Wiley.

Solon, Olivia. 2017. "Ex-Facebook president Sean Parker: site made to exploit human 'vulnerability' ". The Guardian. November 9, 2017.

https://www.theguardian.com/technology/2017/nov/09/facebook-sean-parkervulnerability-brain-psychology.

Soojung-Kim Pang, Alex. 2013. The Distraction Addiction: Getting the Information You Need and the Communication You Want, Without Enraging Your Family, Annoying Your Colleagues, and Destroying Your Soul. New York: Little, Brown and Company.

Stachurski, Christina. 2009. "Man Alone." Cross / Cultures (109): 1-35. 
Stanfill, Mel. 2014. "The interface as discourse: The production of norms through web design." New Media \& Society 17 (7): 1059-1074. https://doi.org/10.1177/1461444814520873.

Stark, Luke. 2018. "Algorithmic psychometrics and the scalable subject." Social Studies of Science 48 (2): 204-231. https://doi.org/10.1177/0306312718772094.

Stibe, Agnis. 2020. "Transforming Technology for Global Business Acceleration and Change Management." Journal of Global Information Technology Management 23 (2): 83-88. https://doi.org/10.1080/1097198X.2020.1752077.

Strauss, Neil. 2011. Everyone Loves You When You're Dead: Journeys Into Fame and Madness. New York: Harper Collins.

Sulleyman, Aatif. 2017. "Nokia 3310 Relaunch: Everything You Need To Know About the Update Classic." Independent. Accessed 26 February, 2017. https://www.independent.co.uk/life-style/gadgets-and-tech/news/nokia-3310relaunch-mwc-2017-need-to-know-specs-price-where-buy-a7597646.html.

Sunstein, Cass R. 2014. Why Nudge? The Politics of Libertarian Paternalism. New Haven: Yale University Press.

Sutton, Theodora. 2017. "Disconnect to reconnect: The food/technology metaphor in digital detoxing." First Monday 22 (6).

https://firstmonday.org/ojs/index.php/fm/article/view/7561.

Sutton, Theodora. 2020. "Digital Harm and Addiction: an Anthropological View." Anthropology Today 36 (1): 17-22. https://doi.org/10.1111/1467-8322.12553.

Syvertsen, Tine, and Gunn Enli. 2019. "Digital detox: Media resistance and the promise of authenticity." Convergence: https://doi.org/10.1177/1354856519847325.

Syvertsen, Tine. 2017. Media Resistance: Protest, Dislike, Abstention. Cham: Palgrave MacMillan.

Tandoc, Edson C. 2014. "Journalism is twerking? How web analytics is changing the process of gatekeeping." New Media \& Society 16 (4): 559-575.

https://doi.org/10.1177/1461444814530541.

Tanti, Adrian, and Dimitrios Buhalis. 2017. "The influences and consequences of being digitally connected and/or disconnected to travellers." Information Technology \& Tourism 17 (1): 121-141. https://doi.org/10.1007/s40558-017-0081-8.

Taplin, Jonathan. 2017. Move Fast and Break Things: How Facebook, Google and Amazon Have Cornered Culture and What It Means For All of Us. London: Pan Macmillan.

Tarnoff, Ben, and Moira Weigel. 2018. "Why Silicon Valley can't fix itself." The Guardian. 3 May, 2018. https://www.theguardian.com/news/2018/may/03/why-silicon-valley-cant-fixitself-tech-humanism. 
Thaler, Richard H, and Cass R Sunstein. 2009. Nudge: Improving decisions about health, wealth, and happiness. Penguin Books.

Thoreau, Henry David. (1854) 1995. Walden; Or, Life In The Woods. New York: Dover Thrift Editions.

Thorén, Claes, Mats Edenius, Jenny Eriksson Lundström, and Andreas Kitzmann. 2017. "The hipster's dilemma: What is analogue or digital in the post-digital society?"

Convergence 25 (2): 324-339. https://doi.org/10.1177/1354856517713139.

Tufekci, Zeynep. 2014. "Engineering the public: Big data, surveillance and computational politics." First Monday 19 (7).

https://firstmonday.org/ojs/index.php/fm/article/view/4901.

Tufekci, Zeynep. 2015. "Algorithmic harms beyond Facebook and Google: Emergent challenges of computational agency." Colorado Technology Law Journa/13: 203-218.

Turkle, Sherry. 2013. Alone Together: Why We Expect More from Technology and Less from Each Other. New York: Basic Books.

Turner, Fred. 2006. From Counterculture to Cyberculture: Stewart Brand, the Whole Earth Network, and the Rise of Digital Utopianism. London: The University of Chicago Press.

Turner, Fred. 2009. "Burning Man at Google: a cultural infrastructure for new media production." New Media \& Society 11 (1-2): 73-94. https://doi.org/10.1177/1461444808099575.

Twenge, Jean M. 2017. iGen: Why Today's Super-Connected Kids Are Growing Up Less Rebellious, More Tolerant, Less Happy--and Completely Unprepared for Adulthood-and What That Means for the Rest of Us. New York: Atria Books.

Ugander, Johan, Brian Karrer, Lars Backstrom, and Cameron Marlow. 2011. "The Anatomy of the Facebook Social Graph." CoRR. http://arxiv.org/abs/1111.4503.

Unyoked. 2019. "Unyoked." Accessed 10 October 2019. https://www.unyoked.co/.

UPLIFT. 2015. "From Burning Man to Intentional Community." UPLIFT. October 7, 2015. https://upliftconnect.com/burning-man-intentional-community/.

Vaidhyanathan, Siva. 2012. The Googlization of Everything: (And Why We Should Worry). Berkeley: University of California Press.

Vaidhyanathan, Siva. 2018. Anti-social Media: How Facebook Disconnects Us and Undermines Democracy. New York: Oxford University Press.

Vakoch, Douglas. 1998. "The therapeutic space of psychoanalysis." Psychoanalysis and Contemporary Thought 21: 335-358.

van Dijck, José, and Thomas Poell. 2013. "Understanding Social Media Logic." Media and Communication 1 (1): 2-14. https://doi.org/10.17645/mac.v1i1.70. 
van Dijck, José. 2013. The Culture of Connectivity: A Critical History of Social Media. New York: Oxford University Press.

van Ooijen, I, and Helena U. Vrabec. 2019. "Does the GDPR Enhance Consumers' Control over Personal Data? An Analysis from a Behavioural Perspective." Journal of Consumer Policy 42 (1): 91-107. https://doi.org/10.1007/s10603-018-9399-7.

Vasagar, Jeeven. 2016. "Kreditech: A credit check by social media." Financial Times, January 20, 2016. https://www.ft.com/content/12dc4cda-ae59-11e5-b955-1a1d298b6250.

Verbeek, Peter-Paul. 2006. "Materializing Morality: Design Ethics and Technological Mediation." Science, Technology, \& Human Values 31 (3): 361-380.

https://doi.org/10.1177/0162243905285847.

Verbeek, Peter-Paul. 2009. "Ambient Intelligence and Persuasive Technology: The Blurring Boundaries Between Human and Technology." Nanoethics 3 (3): 231-242. https://doi.org/10.1007/s17569-009-0077-8.

Verbeek, Peter-Paul. 2011. Moralizing Technology: Understanding and Designing the Morality of Things. Chicago: The University of Chicago Press.

VikingCodeSchool. 2020. "Your Own UX Teardown." VikingCodeSchool. https://www.vikingcodeschool.com/web-design-basics/your-own-ux-teardown.

Vincent, James. 2017. "Former Facebook exec says social media is ripping apart society." The Verge. December 11, 2017.

https://www.theverge.com/2017/12/11/16761016/former-facebook-exec-rippingapart-society.

Virno, Paolo. 1996. "Virtuosity and Revolution: The Political Theory of Exodus." In Radical Thought in Italy: A Potential Politics, edited by Paolo Virno and Michael Hardt, 13-37. Minneapolis: University of Minnesota Press.

Vonderau, Patrick. 2017. "The Spotify Effect: Digital Distribution and Financial Growth." Television \& New Media 20 (1): 3-19. https://doi.org/10.1177/1527476417741200.

Wajcman, Judy. 2015. Pressed for Time: The Acceleration of Life in Digital Capitalism. Chicago: The University of Chicago Press.

Wajcman, Judy. 2019. "How Silicon Valley sets time." New Media \& Society 21 (6): 1272-1289. https://doi.org/10.1177/1461444818820073.

Wakefield, Melanie A, Linda Hayes, Sarah Durkin, and Ron Borland. 2013. "Introduction effects of the Australian plain packaging policy on adult smokers: a cross-sectional study." BMJ Open 3 (7). https://doi.org/10.1136/bmjopen-2013-003175.

Walls, Laura Dassow. 1993. "'Walden" as Feminist Manifesto." Interdisciplinary Studies in Literature and Environment 1 (1): 137-144. http://www.jstor.org/stable/44085351. 
Wark, Scott. 2019. "The subject of circulation: on the digital subject's technical individuations." Subjectivity 12 (1): 65-81. https://doi.org/10.1057/s41286-018-00062$\underline{5}$.

Warschauer, Mark. 2004. Technology and Social Inclusion: Rethinking the Digital Divide. Cambridge, MA: MIT Press.

Watson, David L, and Roland W Tharp. 2013. Self-Directed Behavior: Self-Modification for Personal Adjustment. 10th ed. New York: Wadsworth Publishing.

Watson-Phillips, Carol. 2016. "Relational Fathering: Sons Liberate Dads." The Journal of Men's Studies 24 (3): 277-293. https://doi.org/10.1177/1060826516661188.

Way, Katie. 2019. "'Dopamine Fasting' Is the Newest 'Sounds Fake, but OK' Wellness Trend." Vice. October 18, 2019. https://www.vice.com/en_us/article/vb5ab9/dopaminefasting-is-the-newest-sounds-fake-but-ok-wellness-trend.

Weeks, Kathi. 2011. The Problem with Work: Feminism, Marxism, Antiwork Politics, and Postwork Imaginaries. Durham: Duke University Press.

Weir, Allison. 2013. Identities and Freedom: Feminist Theory Between Power and Connection. Oxford Scholarship Online.

Weiser, Mark, and John Seely Brown. 1995. "Designing Calm Technology." Xerox PARC. December 21, 1995. https://calmtech.com/papers/designing-calm-technology.html.

Wendel, Stephen. 2014. Designing for Behavior Change: Applying Psychology and Behavioural Economics. Sebastopol: O'Reilly.

Wheatley, Dawn, and Eirik Vatnoey. 2020. "Understanding attitudes towards social media segregation: spatial metaphors in the discussion of Twitter blocklists." Information, Communication \& Society. 1-16. https://doi.org/10.1080/1369118X.2020.1749696.

White, Martha. 2012. "Does Not Having a Facebook Page Make You 'Suspicious' to Employers?" Time. August 8, 2018. http://business.time.com/2012/08/08/does-nothaving-a-facebook-page-make-you-suspicious-to-employers/.

Williams, James. 2018. Stand Out Of Our Light: Freedom and Resistance in the Attention Economy. Cambridge, UK: Cambridge University Press.

Wills, M. 1999. "Bridging the digital divide." Adults Learning. 10-11.

Wilson, A. 1987. "The information rich and the information poor." ASLIB Proc 39 (1): 1-6.

Wojdynski, Bartosz W. 2016. "The Deceptiveness of Sponsored News Articles: How Readers Recognize and Perceive Native Advertising." American Behavioral Scientist 60 (12): 1475-1491. https://doi.org/10.1177/0002764216660140. 
Woodstock, Louise. 2014. "Media Resistance: Opportunities for Practice Theory and New Media Research." International Journal of Communication 8.

https://ijoc.org/index.php/ijoc/article/view/2415.

Wright, Randall S. 2017. "Strategy Inversion: Engaging with the Counterculture." ResearchTechnology Management 60 (2): 53-54. https://doi.org/10.1080/08956308.2017.1276391.

Wu, Tim. 2016. The Attention Merchants: The Epic Scramble to Get Inside Our Heads. New York: Vintage.

Wyatt, Sally, Graham Thomas, and Tiziana Terranova. 2002. "They came, they surfed, they went back to the beach: conceptualising use and non-use of the Internet." In Virtual society? Technology, cyberpole, reality, edited by Steve Woolgard, 23-40. Oxford: Oxford University Press.

Wyatt, Sally. 2014. "Bringing users and non-users into being across methods and disciplines." In Proceedings of refusing, limiting, departing (CHI '74 Workshop). Toronto, 26 April-1 May.

Yeung, Karen. 2017. "'Hypernudge': Big Data as a mode of regulation by design." Information, Communication \& Society 20 (1): 118-136. https://doi.org/10.1080/1369118X.2016.1186713.

Young, Sherman. 1998. "Of cyber spaces: The Internet \& heterotopias." M/C Journa/ 1 (4). http://journal.media-culture.org.au/9811/hetero.php.

Zamora, Daniel. 2019a. "Finding a "Left Governmentality": Foucault's Last Decade." In Foucault, Neoliberalism, and Beyond, edited by Stephen W. Sawyer, and Daniel Steinmetz-Jenkins, 53-72. London: Rowman \& Littlefield.

Zamora, Daniel. 2019b. "Foucault, The End of a Decade." Contemporary European History 28 (2): 262-272. https://doi.org/10.1017/s0960777318000577.

Zandbergen, Dorien. 2012. "Fulfilling the Sacred Potential of Technology: New Edge Technophilia, Consumerism and Spirituality in Silicon Valley." In Things: Material Religion and the Topography of Divine Space, edited by Dick Houtman and Birgit Meyer, 356-379. New York: Fordham University Press.

Zuboff, Shoshana. 2019. The Age of Surveillance Capitalism: The Fight for a Human Struggle at the New Frontier of Power. London: Profile Books.

Zuckerberg, Mark. 2018. Facebook. January 11, 2018.

https://www.facebook.com/zuck/posts/10104413015393571. 


\section{Appendix One: Interview Topic List}

\section{Topic 1: Introduction}

- Introduce yourself and the research project

- Ask the interviewee to introduce her/himself

- Age, gender, ethnicity/race

- Place of residence, place of birth

- Profession, level of education

- Provide interviewee with information sheet and consent form

\section{Topic 2: 'Tech culture'}

- Ask the interviewee about:

- what terms such as 'Silicon Valley culture', 'tech culture' or the 'attention economy' mean to them

- the societal impact(s) of Silicon Valley or 'tech culture'

- opinions and/or philosophies regarding the social and/or health role of technology, and

○ the relationship between technology and disconnection.

\section{Topic 3: Disconnection}

- Ask the interviewee about:

- opinions and/or philosophies towards disconnection

- motivations to disconnect

- benefits of disconnecting (such as in work and/or leisure contexts)

- organisation mission statements or governance models regarding disconnection (if applicable)

- any other personal meanings regarding disconnection

\section{Topic 4: Walkthrough of technologies of disconnection}

- Ask the interviewee to discuss:

- The context/background behind their technology of disconnection

- How the technology of disconnection technically works

- Business models (if applicable)

- Feedback from users, and

- Organisation/product goals or targets

\section{Topic 5: Conclusion}

- $\quad$ Thank the interviewee for participating

- Inform the interviewee of next steps and research timelines 